\title{
Single Cell Analysis in Biotechnology and Systems Biology
}

\section{Edited by}

Fan-Gang Tseng and Tuhin Subhra Santra

Printed Edition of the Special Issue Published in International Journal of Molecular Sciences 
Fan-Gang Tseng and Tuhin Subhra Santra (Eds.)

\section{Single Cell Analysis in Biotechnology and Systems Biology}


This book is a reprint of the Special Issue that appeared in the online, open access journal, International Journal of Molecular Sciences (ISSN 1422-0067) in 2015 (available at: http://www.mdpi.com/journal/ijms/special_issues/single_cell).

Guest Editors

Fan-Gang Tseng

National Tsing Hua University

Taiwan

Tuhin Subhra Santra

Indian Institute of Technology Madras

India

Editorial Office

MDPI AG

Klybeckstrasse 64

Basel, Switzerland

Publisher

Shu-Kun Lin

Managing Editor

Rui Liu

\section{Edition 2016}

MDPI $\bullet$ Basel $\bullet$ Beijing $\bullet$ Wuhan $\bullet$ Barcelona

ISBN 978-3-03842-193-1 (Hbk)

ISBN 978-3-03842-194-8 (PDF)

(C) 2016 by the authors; licensee MDPI, Basel, Switzerland. All articles in this volume are Open Access distributed under the Creative Commons Attribution license (CC BY), which allows users to download, copy and build upon published articles even for commercial purposes, as long as the author and publisher are properly credited, which ensures maximum dissemination and a wider impact of our publications. However, the dissemination and distribution of physical copies of this book as a whole is restricted to MDPI, Basel, Switzerland. 


\section{Table of Contents}

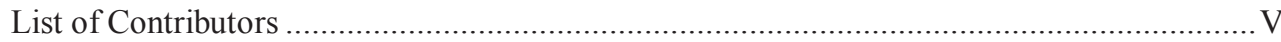

About the Guest Editors.............................................................................................. VII

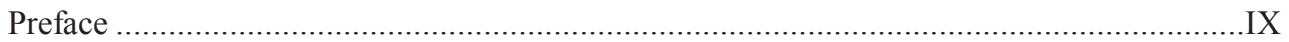

Andre Gross, Jonas Schoendube, Stefan Zimmermann, Maximilian Steeb,

Roland Zengerle and Peter Koltay

Technologies for Single-Cell Isolation

Reprinted from: Int. J. Mol. Sci. 2015, 16(8), 16897-16919

http://www.mdpi.com/1422-0067/16/8/16897. 1

\section{Kjetil Hodne and Finn-Arne Weltzien}

Single-Cell Isolation and Gene Analysis: Pitfalls and Possibilities

Reprinted from: Int. J. Mol. Sci. 2015, 16(11), 26832-26849

http://www.mdpi.com/1422-0067/16/11/25996 .25

\section{Jie-Long He, An-Te Chen, Jyong-Huei Lee and Shih-Kang Fan}

Digital Microfluidics for Manipulation and Analysis of a Single Cell

Reprinted from: Int. J. Mol. Sci. 2015, 16(9), 22319-22332

http://www.mdpi.com/1422-0067/16/9/22319.

\section{Amelia Ahmad Khalili and Mohd Ridzuan Ahmad}

Numerical Analysis of Hydrodynamic Flow in Microfluidic Biochip for Single-Cell

Trapping Application

Reprinted from: Int. J. Mol. Sci. 2015, 16(11), 26770-26785

http://www.mdpi.com/1422-0067/16/11/25987.

\section{Shih-Jie Lo and Da-Jeng Yao}

Get to Understand More from Single-Cells: Current Studies of Microfluidic-Based Techniques for Single-Cell Analysis

Reprinted from: Int. J. Mol. Sci. 2015, 16(8), 16763-16777

http://www.mdpi.com/1422-0067/16/8/16763. 
Authors Muhammad Asraf Mansor and Mohd Ridzuan Ahmad

Single Cell Electrical Characterization Techniques

Reprinted from: Int. J. Mol. Sci. 2015, 16(6), 12686-12712

http://www.mdpi.com/1422-0067/16/6/12686.

.97

Jian Chen, Chengcheng Xue, Yang Zhao, Deyong Chen, Min-Hsien Wu and Junbo Wang

Microfluidic Impedance Flow Cytometry Enabling High-Throughput Single-Cell Electrical

Property Characterization

Reprinted from: Int. J. Mol. Sci. 2015, 16(5), 9804-9830

http://www.mdpi.com/1422-0067/16/5/9804

Chansavath Phetsouphanh, John James Zaunders and Anthony Dominic Kelleher

Detecting Antigen-Specific T Cell Responses: From Bulk Populations to Single Cells

Reprinted from: Int. J. Mol. Sci. 2015, 16(8), 18878-18893

http://www.mdpi.com/1422-0067/16/8/18878.

Jennifer Pasquier, Damien Rioult, Nadine Abu-Kaoud, Jessica Hoarau-Véchot, Matthieu Marin and Frank Le Foll

Evidence for P-Glycoprotein Involvement in Cell Volume Regulation Using Coulter Sizing in Flow Cytometry

Reprinted from: Int. J. Mol. Sci. 2015, 16(7), 14318-14337

http://www.mdpi.com/1422-0067/16/7/14318. 168

\section{Amelia Ahmad Khalili and Mohd Ridzuan Ahmad}

A Review of Cell Adhesion Studies for Biomedical and Biological Applications

Reprinted from: Int. J. Mol. Sci. 2015, 16(8), 18149-18184

http://www.mdpi.com/1422-0067/16/8/18149. 188 


\section{List of Contributors}

Nadine Abu-Kaoud: Stem Cell and Microenvironment Laboratory, Weill Cornell Medical College in Qatar, Education City, Qatar Foundation, Doha 24144, Qatar.

Mohd Ridzuan Ahmad: Faculty of Electrical Engineering, Universiti Teknologi Malaysia, 81310-UTM Skudai, Johor, Malaysia; Department of Control and Mechatronic Engineering, Faculty of Electrical Engineering, Institute of Ibnu Sina, Universiti Teknologi Malaysia, Skudai, Johor 81310, Malaysia.

Jian Chen: State Key Laboratory of Transducer Technology, Institute of Electronics, Chinese Academy of Sciences, Beijing 100190, China.

Deyong Chen: State Key Laboratory of Transducer Technology, Institute of Electronics, Chinese Academy of Sciences, Beijing 100190, China.

An-Te Chen: Department of Mechanical Engineering, National Taiwan University, Taipei 10617, Taiwan.

Shih-Kang Fan: Department of Mechanical Engineering, National Taiwan University, Taipei 10617, Taiwan.

Frank Le Foll: Laboratory of Ecotoxicology UPRES EA 3222, IFRMP 23, University of Le Havre, 76058 Le Havre, France.

Andre Gross: Laboratory for MEMS Applications, IMTEK-Department of Microsystems Engineering, University of Freiburg, Georges-Koehler-Allee 103, Freiburg 79110, Germany; Cytena GmbH, Georges-Koehler-Allee 103, Freiburg 79110, Germany.

Jie-Long He: Department of Mechanical Engineering, National Taiwan University, Taipei 10617, Taiwan.

Jessica Hoarau-Véchot: Stem Cell and Microenvironment Laboratory, Weill Cornell Medical College in Qatar, Education City, Qatar Foundation, Doha 24144, Qatar.

Kjetil Hodne: Department of Basic Sciences and Aquatic Medicine, Norwegian University of Life Sciences_Campus Adamstuen, 0033 Oslo, Norway.

Anthony Dominic Kelleher: Kirby Institute, University of New South Wales, 2031 Sydney, Australia; Centre for Applied Medical Research, St. Vincent's Hospital, 2010 Sydney, Australia.

Amelia Ahmad Khalili: Department of Control and Mechatronic Engineering, Faculty of Electrical Engineering, Universiti Teknologi Malaysia, Skudai, Johor 81310, Malaysia.

Peter Koltay: Laboratory for MEMS Applications, IMTEK-Department of Microsystems Engineering, University of Freiburg, Georges-Koehler-Allee 103, Freiburg 79110, Germany; Cytena GmbH, Georges-Koehler-Allee 103, Freiburg 79110, Germany.

Jyong-Huei Lee: Department of Mechanical Engineering, National Taiwan University, Taipei 10617, Taiwan.

Shih-Jie Lo: Institute of Nanoengineering and Microsystem, National Tsing Hua University, Hsinchu 300, Taiwan.

Authors Muhammad Asraf Mansor: Faculty of Biosciences and Medical Engineering, Universiti Teknologi Malaysia, 81310-UTM Skudai, Johor, Malaysia. 
Matthieu Marin: UGSF, UMR CNRS 8576, équipe Régulation des Signaux de Division, Université de Lille, Sciences et Technologies, 59009 Villeneuve d'Ascq, France.

Jennifer Pasquier: Stem Cell and Microenvironment Laboratory, Weill Cornell Medical College in Qatar, Education City, Qatar Foundation, Doha 24144, Qatar; Department of Genetic Medicine, Weill Cornell Medical College, New York, NY 10022, USA.

Chansavath Phetsouphanh: Kirby Institute, University of New South Wales, 2031 Sydney, Australia.

Damien Rioult: UMR_I 02 INERIS-URCA-ULH SEBIO Unité Stress Environnementaux et BIOsurveillance des milieux aquatiques, Université de Reims Champagne-Ardenne, BP-1039-Reims Cedex 2, 51687 Reims, France.

Jonas Schoendube: Laboratory for MEMS Applications, IMTEK-Department of Microsystems Engineering, University of Freiburg, Georges-Koehler-Allee 103, Freiburg 79110, Germany; Cytena GmbH, Georges-Koehler-Allee 103, Freiburg 79110, Germany.

Maximilian Steeb: Laboratory for MEMS Applications, IMTEK-Department of Microsystems Engineering, University of Freiburg, Georges-Koehler-Allee 103, Freiburg 79110, Germany.

Junbo Wang: State Key Laboratory of Transducer Technology, Institute of Electronics, Chinese Academy of Sciences, Beijing 100190, China.

Finn-Arne Weltzien: Department of Basic Sciences and Aquatic Medicine, Norwegian University of Life Sciences - Campus Adamstuen, 0033 Oslo, Norway.

Min-Hsien Wu: Graduate Institute of Biochemical and Biomedical Engineering, Chang Gung University, Taoyuan 333, Taiwan.

Chengcheng Xue: State Key Laboratory of Transducer Technology, Institute of Electronics, Chinese Academy of Sciences, Beijing 100190, China.

Da-Jeng Yao: Institute of Nanoengineering and Microsystem, National Tsing Hua University, Hsinchu 300, Taiwan.

John James Zaunders: Kirby Institute, University of New South Wales, 2031 Sydney, Australia; Centre for Applied Medical Research, St. Vincent's Hospital, 2010 Sydney, Australia.

Roland Zengerle: Laboratory for MEMS Applications, IMTEK-Department of Microsystems Engineering, University of Freiburg, Georges-Koehler-Allee 103, Freiburg 79110, Germany; Hahn-Schickard, Georges-Koehler-Allee 103, Freiburg 79110, Germany; BIOSS-Centre for Biological Signalling Studies, University of Freiburg, Freiburg 79110, Germany.

Yang Zhao: State Key Laboratory of Transducer Technology, Institute of Electronics, Chinese Academy of Sciences, Beijing 100190, China.

Stefan Zimmermann: Laboratory for MEMS Applications, IMTEK-Department of Microsystems Engineering, University of Freiburg, Georges-Koehler-Allee 103, Freiburg 79110, Germany. 


\section{About the Guest Editors}

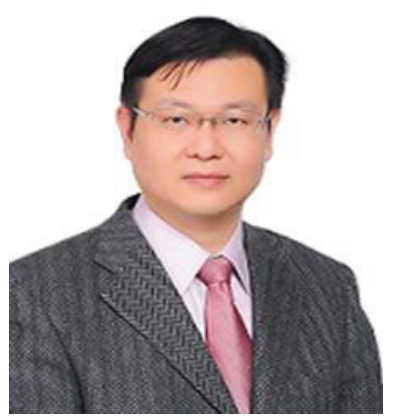

Dr. Fan-Gang (Kevin) Tseng received his $\mathrm{PhD}$ in Microelectro Mechanical System (MEMS) from the University of California, Los Angeles, USA (UCLA), under the supervision of Professor C-M. Ho and C.J. Kim. Dr. Tseng joined as an Assistant Professor in the Department of Engineering and System Science, National Tsing Hua University, Taiwan in August 1999. Currently he is a Distinguished Professor in the Department of Engineering and System Science (ESS) and the Dean of Nuclear Science College at National Tsing Hua University, Taiwan. He has also been a Deputy Director of the Biomedical Technology Research Center, National Tsing-Hua University since 2009. Further, he is also an affiliated Professor at the Institute of Nanoengineering and Microsystems (NEMS), National Tsing Hua University and a Research Fellow in Academia Sinica, Taiwan. Dr. Tseng focuses his research in the following areas: MEMS, bio-MEMS, nano-biotechnology, nanomedicine, fuel cell, MEMS packaging, and integration. Dr. Tseng has been on the Editorial Board of Applied Sciences from 2010, the Journal of Circuits and Systems from 2010, the Open Micromachine Journal from 2009, the Open Nanomedicine Journal from 2008, the MEMS special issue, Electronic Magazine from 2009, the Chinese Micro Electromechanical System magazine in 2002 and 2004. He was the editor of a book entitled "Micro Electromechanical System Technology and Applications", published by the Precision Instrument Development Center of National Science Council, in 2003. Dr. Tseng was elected an ASME fellow in 2014, and received several awards, including Outstanding in Research Awards (2010 and 2014) and Mr. Wu, Da-Yo Memorial Award (2005) from MOST, Taiwan, National Innovation Award in 2014, nine Best Paper/Poster awards (1991, 2003, 2004, 2005, 2008, 2010, 2012, 2013, 2014), among others. He has received 40 patents, written eight books/book chapters, published more than 200 SCI Journal papers and 350 conference technical papers in biosensors, bio-N/MEMS, micro fuel cells, and micro/nano fluidics related fields, and coorganized or co-chaired many conferences including IEEE MEMS, IEEE NEMS, IEEE Transducers, Micro TAS, ISMM, IEEE Nano, and IEEE Nanomed. 


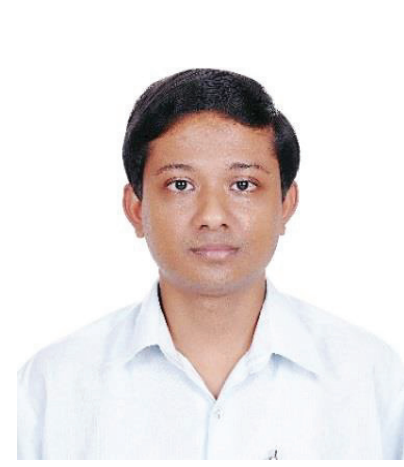

Dr. Tuhin Subhra Santra received his $\mathrm{PhD}$ degree in Bio-Nano Electro Mechanical Systems (Bio-NEMS), especially for single cell analysis from the Institute of Nanoengineering and Microsystems (NEMS), National Tsing Hua University (NTHU), Taiwan in 2013, under the supervision of Professor Fan-Gang Tseng. Dr. Santra joined as an Assistant Professor in the Indian Institute of Technology Madras, India in July 2016. His main research areas are bioNEMS, MEMS, single cell analysis, bio-micro/nano fabrication, biomedical microdevices, nanomedicine. Dr. Santra serves as a Guest Editor for the Journal of Micromachines, Sensors Journal, Journal of Molecules, International Journal of Molecular Science, Sensors and Transducers Journal, American Journal of Nanoresearch and Application, among others. Dr. Santra has received many awards such as the NTHU outstanding student award in 2011 and 2013, IEEE-NEMS best conference paper award in 2014, best poster award at 15th Nano/Microsystem Conference in 2012, Junior Research Fellowship at IIT-Kharagpur in 2008, outstanding student awards at NTHU and Jadavpur University in 2005, 2006, 2010 and 2012, silver medal from Vidyasagar University in 2004. Dr. Santra has published more than 15 international journal papers, has one US and Taiwan pending patents, three books, six book chapters, six proceedings and 25 International Conference papers in his research field. 


\section{Preface}

Cells are the most fundamental building blocks of most life forms and play a significant role in coordinating with each other to perform systematic functions in all living creatures. However, the behavior of cell to cell, or cell to the environment with their organelles and their intracellular physical and biological functions remains unknown. To better understand the physiological interactions among molecules, organelles, and cells ensemble average measurement of millions of cells together, cannot provide detailed information such as stem cell proliferation, differentiation, neural network coordination, and cardiomyocytes synchronization. Again, biological functions such as genomes, epigenomes and transcriptomes as a bulk population is informative, however it is not enough to understand the cellular heterogeneity characteristics in phenotypic behavior assays and dynamics of individual cells. Thus, single cells analysis (SCA) has evolved in frontier research.

Over the last two decades there has been a tremendous shift to study biological cell function in a holistic manner rather than as a reductionist scientific paradigm, thus establishing the approach to be named "systems biology" or "systomics". In this system, multiple disciplines including biochemistry, molecular biology, physics, mathematics, information technology and system engineering act together to examine the interaction between biological pathways rather than individual pathways in an isolated manner. Today system biology can study individual characteristics of single cells conducted by employing miniaturized devices, whose dimensions are similar to that of single cells.

Due to the rapid development of sophisticated micro/nanofluidic devices, we now have Bio-MEMS, Lab on a Chip (LOC), and micro total analysis systems ( $\mu$ TAS) that enable more complex manipulations of chemicals and biological agents in fluidic environments. Thus, micro-nanofluidic devices are not only useful for cell isolation, manipulation, cell lysis, and cell separation, but also to easily control the biochemical, mechanical and electrical parameters of SCA. With these novel devices, technology has become a pioneer in omics analyses and an integral part of medical biotechnology, such as diagnostics, prognostics and cancer therapy.

This special issue book entitled "Single Cell Analysis in Biotechnology and System Biology" summarizes an overview of single cell manipulation, isolation, injection, cell lysis, intracellular delivery with electrokinetic phenomenon, transport mechanisms, flow resistance and molecular diffusion into cells. The book also discusses biochemical, mechanical and electrical characterization of single cell by using advanced micro/nanofluidic devices. The role of SCA in system biology, proteomics, genomics and the applications of SCA in medical biotechnology with future challenges and their advantages as well as limitations are also elaborated.

We hope this book will be valuable for the academic and industrial scientific community, where researchers are working on SCA with different clinical applications.

Fan-Gang Tseng and Tuhin Subhra Santra

Guest Editors 



\title{
Technologies for Single-Cell Isolation
}

\section{Andre Gross, Jonas Schoendube, Stefan Zimmermann, Maximilian Steeb, Roland Zengerle and Peter Koltay}

\begin{abstract}
The handling of single cells is of great importance in applications such as cell line development or single-cell analysis, e.g., for cancer research or for emerging diagnostic methods. This review provides an overview of technologies that are currently used or in development to isolate single cells for subsequent single-cell analysis. Data from a dedicated online market survey conducted to identify the most relevant technologies, presented here for the first time, shows that FACS (fluorescence activated cell sorting) respectively Flow cytometry (33\% usage), laser microdissection (17\%), manual cell picking (17\%), random seeding/dilution (15\%), and microfluidics/lab-on-a-chip devices $(12 \%)$ are currently the most frequently used technologies. These most prominent technologies are described in detail and key performance factors are discussed. The survey data indicates a further increasing interest in single-cell isolation tools for the coming years. Additionally, a worldwide patent search was performed to screen for emerging technologies that might become relevant in the future. In total 179 patents were found, out of which 25 were evaluated by screening the title and abstract to be relevant to the field.
\end{abstract}

Reprinted from Int. J. Mol. Sci. Cite as: Gross, A.; Schoendube, J.; Zimmermann, S.; Steeb, M.; Zengerle, R.; Koltay, P. Technologies for Single-Cell Isolation. Int. J. Mol. Sci. 2015, 16, 16897-16919.

\section{Introduction}

With regards to heterogeneous cell populations, such as those found in many tumors, the generation and analysis of single cells has an increasing impact on various fields of life sciences and biomedical research [1]. The analysis of heterogeneous cell populations in bulk is only able to provide averaged data about the population, by which important information about a small but potentially relevant subpopulation is possibly lost in the background. Cancer development is based on a complex interrelation of mutations, selection, and clonal expansion resulting in a mosaic out of different subclones within a single tumor [2]. If rare subclones, which lead to only subtle genomic signals, can be detected at all from studying bulk populations, it takes a large sequencing and computational effort [3]. In contrast, the analysis of single cells, representing such a subpopulation, can provide very detailed information - information which may be used for therapeutic decisions in an increasingly personalized medicine. A further need for the analysis of single-cells relates to very rare cells like circulating tumor cells, which are surrounded by billions of normal blood cells and have an increasing clinical impact as a so-called liquid biopsy [4]. However, at present, the isolation and separation of single cells is still a technically challenging task. Main challenges are the yield and quality or in other words the integrity and purity of the cells as well as the throughput and the sensitivity of single cell isolation methods. Today, a large variety of technologies for single-cell separation, isolation, and 
sorting are already available that are applied according to the scientific objective. These technologies can be briefly classified according to their:

- Level of automation, distinguishing manual methods for cell separation like microscope-assisted picking from automated devices such as fluorescence activated cell sorters (FACS).

- Ability to isolate specific/individual cells, distinguishing statistical methods (i.e., the separation of cells according to a certain statistical probability) from a controlled cell separation (i.e., a cell is specifically selected and confirmed to be single).

- Compatibility with certain application requirements, distinguishing technologies mainly applied for production of monoclonal cell cultures (derived from single cells) from technologies preferably used for single-cell genome/proteome analysis.

In this review, we present the current single-cell isolation technologies in consideration of their compatibility with requirements for downstream life-science applications. In order to identify the most relevant technologies to be presented, a market study of single-cell technologies was conducted by the authors by means of an online survey. After briefly describing the methodology of the market study, the five most-used technologies are reviewed in the following in detail. In addition to these most widely adopted technologies, further emerging technologies were identified through a patent search at the European Patent Office (EPO) for worldwide patents about single-cell separation technologies. Many of these patented technologies are probably not commercialized so far, but nevertheless have the potential to enable advances in the field of single-cell research and might become more relevant and well known in the future. Although this review does not claim to be exhaustive, it might turn out be a helpful guide through the heterogeneous field of state-of-the-art single-cell handling technologies.

\section{Market Study of Single-Cell Technologies}

As part of this work, a market survey about the German market for single-cell technologies has been conducted in the summer of 2014. More than 3000 contacts of potential survey participants have been manually selected as statistical population as described in the following. The criterion for (academic) research contacts to be considered for participation was to be active in the field of cell biology. For company contacts, the criterion was to be listed in the publicly available database for biotechnological companies "Biotechnology Database", provided by BIOCOM AG, Berlin, Germany [5].

The data for the marked study was generated by an online questionnaire in June and July 2014. Invitations were sent by e-mail to the previously identified group of contacts. In total 210 participants from German universities, research institutes, and industry have responded to the invitation, out of which 102 have completely filled out the questionnaire. Of all participants $17 \%$ are affiliated to universities, $16 \%$ to university hospitals, $11 \%$ to non-university research organizations, $5 \%$ to commercial companies and $51 \%$ did not specify their affiliation. Most participants categorized themselves as belonging to the fields of research in medicine (46\%) or biology (42\%). Figure 1 shows the distribution of the participants in their general and specific fields of research. Basic research appears to be slightly more widespread than applied research, which can potentially be attributed to the 
immaturity of the young field of single-cell research. With regards to the more specific research fields, immunology and oncology were the areas, where most participants worked.

(a)

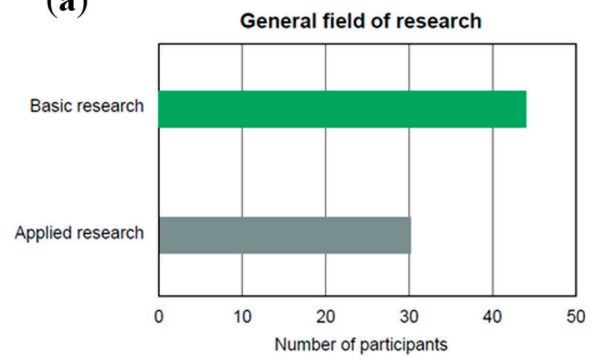

(b)

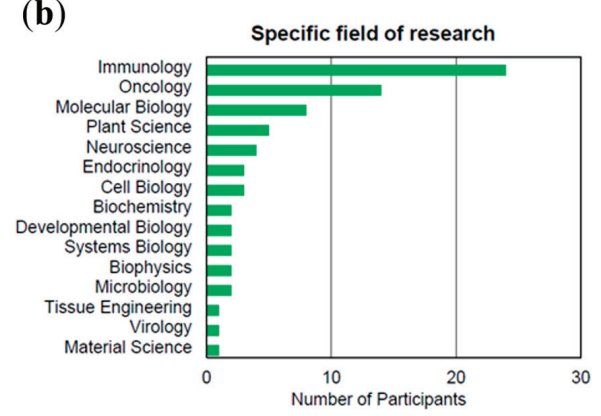

Figure 1. (a) Distribution of participants in their general fields of research. 44 participants stated basic research and 30 applied research as their general field; and (b) Distribution of participants in their specific fields of research. Immunology and oncology were most frequently named.

According to the survey participants, the most commonly used technologies for handling of single cells today are FACS respectively flow cytometry (33\%), manual cell picking (17\%), laser microdissection (also 17\%), random seeding/limiting dilution (15\%) and microfluidics/lab-on-a-chip devices (12\%). Technologies like optical tweezers and others were mentioned less often (in total $6 \%$ ). Figure 2a shows the most commonly used technologies in Germany in 2014. The five most prominent technologies will be in the focus of Section 3 of this review. Comparable results were obtained by a worldwide market study performed also in 2014 by HTStec (Cambridge, UK) [6], in collaboration with the authors of this paper. This study ranks the same technologies as the top five most extensively used amongst researchers worldwide (Figure $2 b$ ).

A further finding of the survey was, that on average approximately 14 single-cell experiments are performed by the respondents per month, which corresponds to 164 experiments per year. The most frequently given answer was 1-5 experiments per month (mode of the data set). This indicates, that single-cell separation and handling is not a routine procedure yet, but performed regularly by those active in the field.

Finally, the participants were asked to rank the importance of the following criteria for selection of a specific instrument for single-cell isolation: acquisition costs, maintenance \& running costs, number of cells needed (minimum to operate the device), cell viability (after isolation), single-cell yield, compatibility with existing workflows, throughput (in terms of single cells per second), and space needed in the laboratory (for the instrument). It turned out, that all of these criteria are considered to be important (i.e., ranking larger 2.5 out of 5). The lowest ranking had space needed in the laboratory (2.75 of 5) and the highest ranking had cell viability and single-cell yield (4.12 of 5). Certainly, the relative importance of these criteria depend on the specific application, but it is for example noteworthy, that cell viability is ranked in average higher than throughput (3.52 of 5$)$. 


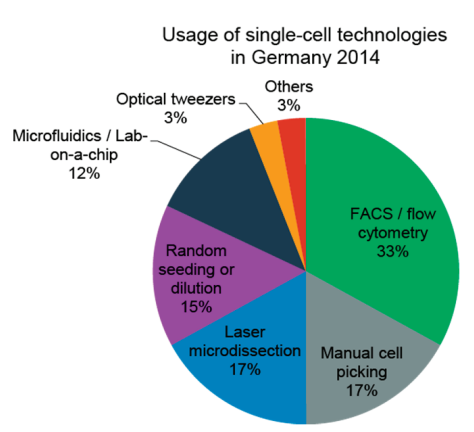

(a)

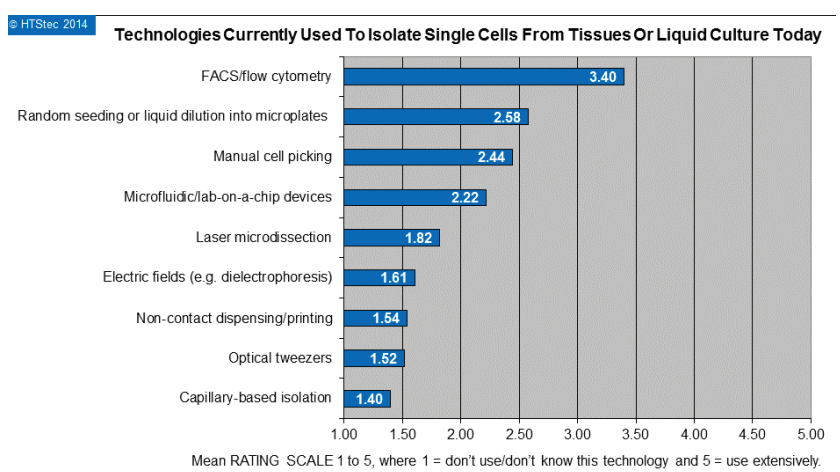

(b)

Figure 2. (a) The usage of technologies for handling single-cells in Germany in 2014. This data was derived as part of this work by a survey amongst 210 participants from German universities, research institutes and industry; and (b) Extensiveness of use of different single-cell technologies (data from "Single Cell Technologies Trends 2014"[6], reproduced with permission from HTStec Limited, Single Cell Technologies Trends 2014, HTStec 2014 URL: http://selectbiosciences.com/ MarketReportsID.aspx?reportID=83).

\section{Single-Cell Isolation Technologies}

Based on the market survey above, the methods and technologies presented hereafter are the most widespread technologies used for single-cell handling. In general, the applied methods strongly depend on the nature and origin of the sample and the processing or analysis to be performed on the cells once being isolated. To illustrate the diversity of sample nature, separation technology, and target applications Figure 3 shows schematics of the working principle of the five methods to be considered in detail in the following.

\subsection{Flow Cytometry}

Thanks to the early pioneers of flow cytometry, since the 1970s researchers have access to ever more powerful flow cytometry instruments. Amongst others, patents and methods developed by Andrew Moldavan 1934 [7], Frank T. Gucker 1947 [8], Wallace H. Coulter 1953 [9], Mark Fulwyler 1965 [10,11], and Wolfgang Dittrich and Wolfgang Göhde 1968 [12] paved the way for the success of commercial flow cytometry [13]. 


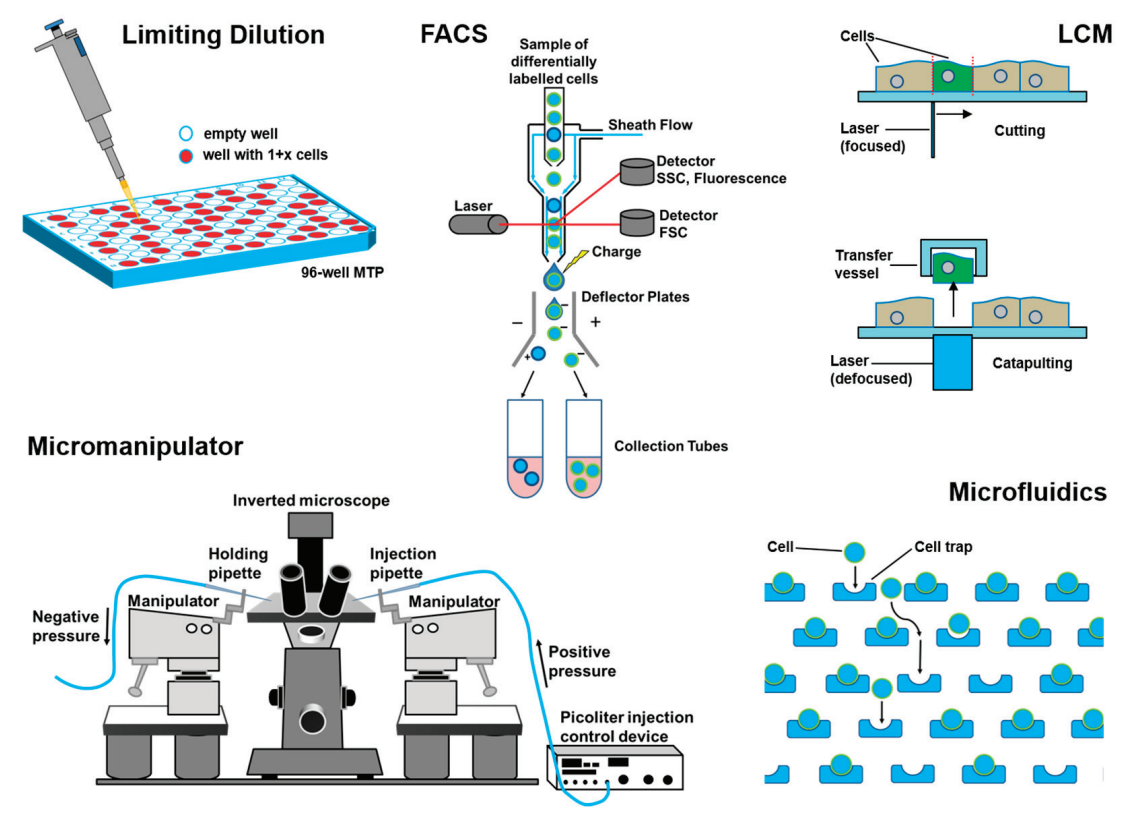

Figure 3. Schematic overview of single-cell separation technologies discussed in the following. The five technologies were identified through market studies as the most commonly used technologies for the handling of single cells ( $c f$. (compare to) Figure 1).

Amongst the various types of flow cytometers, mainly Fluorescence Activated Cell Sorting (FACS) systems provide the ability to isolate single cells, thus they are focus of this section. FACS systems employ laser excitation and offer various analysis options. Cellular properties like relative size and granularity can be extracted as forward scatter (FSC) and side scatter (SSC), respectively. In addition a huge palette of functional properties can be measured by fluorescent staining. In FACS systems, cell suspensions are pressure driven through a flow cell. There they are lined up by a sheath flow liquid exploiting the effect of so called hydrodynamic focusing (see Figure 3). Upon such an arrangement, the cell stream rapidly passes by a laser beam to provide optical excitation and then optical detectors are used downstream to capture cell specific signals. The signals depend on the cells' respective physical, chemical, or optical properties-often enhanced by synthetic markers such as fluorescent dyes. Apart from size analysis and counting, the bypassing cells can also be sorted. After analysis, the cells are suspended in a closed system of small channels, the cell stream is forced through a small nozzle (typically $60-100 \mu \mathrm{m}$ orifice diameter) and thereby a liquid jet is formed. By targeted vibrational actuation (e.g., by ultrasound) this jet breaks apart into a continuous stream of free flying droplets some of which carry cells. Using electrically charged plates for deflection of droplets containing cells of interest, these droplets can be guided to a collector vessel (typically a tube or micro well plate).

Popular systems like the FACS-Aria ${ }^{\mathrm{TM}}$ III (Becton, Dickinson and Company, Franklin Lakes, NJ, USA) provide up to six different colored excitation lasers and simultaneous fluorescent read-out in up to 18 color channels (FACS-Aria III brochure, BD, 2015, http://static.bdbiosciences.com/documents/ 
BD_FACSAria_III_brochure.pdf). The system is able to generate up to 100,000 droplets per second and analyze up to 70,000 events per second (FACSAria III technical data sheet, BD, 2015, http://static.bdbiosciences.com/documents/ BD_FACSAria_III_tech_specs.pdf). Similar systems are available from Beckman Coulter (Brea, CA, USA), Sony Biotechnology Inc. (San Jose, CA, USA.), Bio-Rad Laboratories Inc. (Hercules, CA, USA), and others. Typically FACS systems provide different sort modes specialized either on high throughput or enrichment or purity. Depending on the application, type of cells, and the chosen sort mode the actual rate of sorted cells per second can strongly differ between some hundred up to several thousand cells.

Not only since the discovery of hybridoma cells by Koehler and Milstein [14] FACS has become an accepted, worldwide standard in analysis and sorting of cell populations [15], especially due to the known potential hazards in cloning by limiting dilution [16]. Prominent fields of research and application for the FACS technology are for example: DNA content analysis, immunophenotyping, quantification of soluble molecules [17], cell cycle analysis, hematopoietic stem cells, apoptosis, quantification of subpopulations [18], microbial analysis [19], and cancer diagnostics [20,21]. The sample range covers nearly every cell type from blood, bone marrow, tumor, plants, protoplasts, yeast, to bacteria and even viruses.

\subsection{Laser Capture Microdissection}

Laser capture microdissection (LCM) is an advanced technique to isolate individual cells or cell compartments from mostly solid tissue samples [22,23]. Figure 4 illustrates three different variations of the working principle. A tissue section is observed through a microscope and the target cell or compartment is visually identified. The operator marks the section to be cut off on the display by drawing a line around it. Along this trajectory the laser cuts the tissue and the isolated cell (or compartment) is-if required-extracted. While the cutting procedure using the laser is usually the same, there are several methods to extract the dissected tissue:

Contact-based extraction is done by laser cutting followed by extraction via adhesion, employing adhesive tube caps or heat-absorbing transfer foils, locally made adhesive by infrared (IR) lasers (cf. in Figure 4a).

Contact-free gravity-assisted microdissection (GAM) is featuring an inversely mounted substrate placed over a collector tube. Once being cut out by the laser, the target cell (or compartment) falls down into the collector ( $c f$. in Figure 4b, Leica LMD7000).

Contact-free laser pressure catapulting (LPC) uses a short defocused laser pulse to ignite a local plasma below the previously cut cell (or compartment) [24,25]. The plasma impulse catapults the cell (or compartment) vertically against gravity into a nearby collector container ( $c f$. in Figure 4c, Zeiss PALM MicroBeam LCM). 
(a)
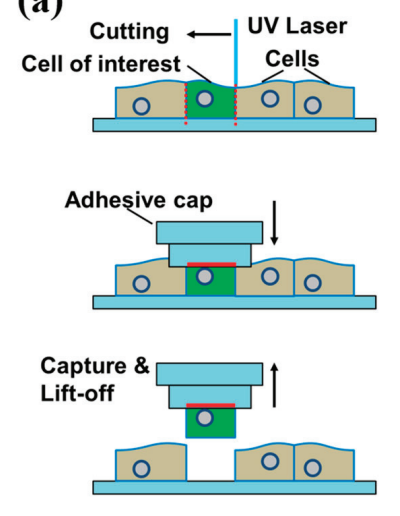

(b)
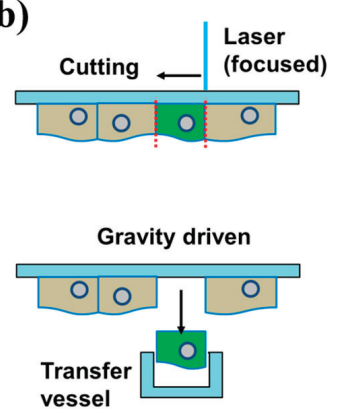

(c)
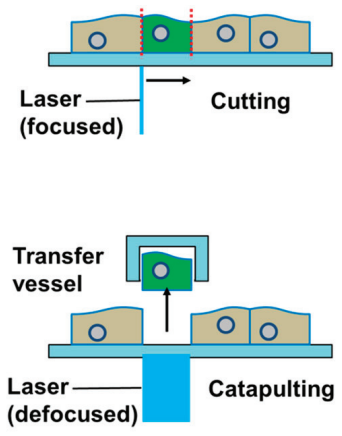

Figure 4. Schematic view on laser capture microdissection (LCM) methods. (a) Contactbased via adhesive tapes; (b) Cutting with a focused laser followed by capture with a vessel. Cut-out section extracted by gravity; and (c) Cutting with a focused laser followed by pressure catapulting with a defocused laser pulse.

Samples are typically provided fixed in formalin, embedded in paraffin, or cryo-fixed [26]. Some LCM systems even allow for dissection of living tissue, enabling the extraction of live cells for culture or analysis (Leica LMD7000 with Live Cell Cutting (LCC)).

Analysis of solid tissue is of great interest when investigating heterogeneous tissue sections regarding their cellular structure as well as physiological and pathological processes [27]. In solid tumor research linking the molecular information of individual cells to their specific location in the tissue has become an important research field. Particularly, the access to cells in situ is of interest [28]. In combination with immune histological staining, LCM is a powerful tool for solid sample analysis on the single-cell level [29]. In the past years, various applications in single-cell analysis based on LCM extracted cells have been published: Single-cell RT-PCR [30], short tandem repeat analysis (STR) analysis in forensics [31], Western blot and mass spectrophotometry [32].

\subsection{Limiting Dilution}

Today many laboratories and companies use hand-pipettes or pipetting robots to isolate individual cells through dilution of the cell suspension. Due to the statistical distribution of the cells in the suspension, the number of cells in a highly diluted sample can be as low as one single cell per aliquot, when the suspension is split into small volumes (aliquots). This process is termed limiting dilution and is well known for decades for the production of monoclonal cell cultures [33-36]. Besides antibody production (as done by hybridomas), other applications such as cell-based assays, etc. also require cell populations grown from a single-cell.

Such seeding of cells in low concentration is indeed simple to carry out with standard pipetting tools, but it is not very efficient since the probability of achieving a single-cell in an aliquot is of statistical nature. The probability to obtain a certain number of cells per aliquot (i.e., 0, 1, 2, etc.) is described by Poisson's distribution [37]. In order to achieve a sufficiently high probability for the 
appearance of single cells while at the same time minimizing the probability for multiple cells, the sample has to be strongly diluted. Typically a density of less than one cell per aliquot is applied (e.g., cell concentration smaller than 1 cell $/ 10 \mu \mathrm{L}=100$ cells $/ \mathrm{mL}$, if the aliquot volume is $10 \mu \mathrm{L}$ ). Often cited protocols e.g., recommend 0.5 to 0.9 cells per aliquot $[16,35,38]$. Table 1 presents the respective distribution of cells per well and the ideally achievable number of empty wells, single cells, and multiple cells according to Poisson's distribution. Obviously, on average only about one third of the prepared wells in a cell culture plate will contain a single-cell. Which of the wells indeed contain single cells has to be confirmed after seeding the cells in a separate process (e.g., by microscopy) due to the statistical nature of the separation method.

Table 1. Statistical probability for the number of cells per aliquot according to Poisson's distribution for cell concentrations of 0.5 and 0.9 cells per aliquot.

\begin{tabular}{cccc}
\hline \multicolumn{2}{c}{ 0.5 Cells/Aliquot } & \multicolumn{2}{c}{ 0.9 Cells/Aliquot } \\
\hline Cell Number/Well & Probability & Cell Number/Well & Probability \\
\hline 0 & $61 \%$ & 0 & $41 \%$ \\
1 & $30 \%$ & 1 & $37 \%$ \\
2 & $8 \%$ & 2 & $16 \%$ \\
3 & $1 \%$ & 3 & $5 \%$ \\
4 & $0 \%$ & 4 & $1 \%$ \\
\hline
\end{tabular}

\subsection{Manual Cell Picking}

Micromanipulators for manual cell picking typically consist of an inverted microscope combined with micro-pipettes movable through motorized mechanical stages. Micropipettes are made of ultrathin glass capillaries coupled to an aspiration and dispensation unit. The cell sample is typically provided as suspension in a dish or well-plate. Via microscope observation the operator selects a specific cell, moves the micro-pipette in close proximity and aspirates the cell by applying suction to the micropipette. The aspirated liquid volume including the selected cell can be transferred to a collection vessel (e.g., a well of a well-plate), where it is released by dispensation. This process is commonly performed manually.

Micromanipulators enable the controlled separation of selected, living cells from suspension and even allow for isolation of prokaryotic cells [39]. The fields of application range from bacterial analysis [40] to reproductive medicine [41] and forensics [42].

\subsection{Microfluidics}

Vast numbers of microfluidic or lab-on-a-chip devices have been proposed for single-cell analysis and handling in the past years (for reviews see [43] or [44]). In this section, we focus on methods that enable isolation of single cells for further downstream analysis or culture. General cell separation techniques that offer no control at the single-cell level [45] are not within the scope of this section. Though many different microfluidic devices for single-cell separation and handling have been published in the literature, most of these devices use at least one of the three following microfluidic principles to isolate single cells: 
- Droplet-in-oil-based isolation as for example published in [46] (Figure 5a);

- Pneumatic membrane valving as for example published in [47] (Figure 5b);

- Hydrodynamic cell traps as for example published in [48] (Figure 5c).

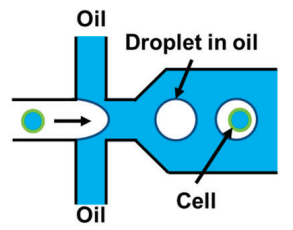

(a)

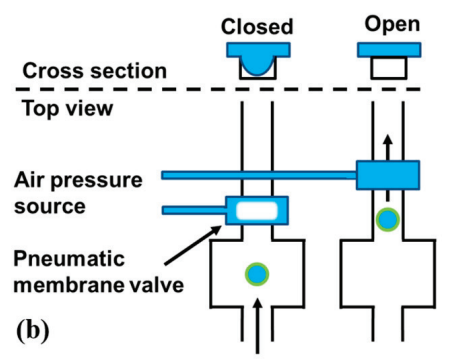

(c)

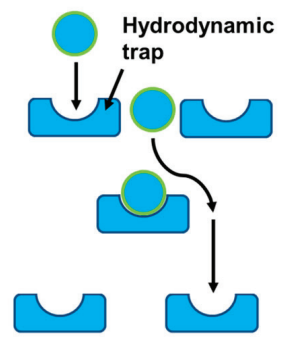

Figure 5. Schematic overview of different microfluidic methods for single-cell isolation. (a) An aqueous stream of cells is broken up into individual droplets-in-oil containing random distribution of cells; (b) Pneumatic membrane valves use air pressure to close a microfluidic channel by membrane deflection. This stops the flow and can trap a cell; and (c) Hydrodynamic traps are passive elements that only fit single cells and hold them at one position.

Droplet-based microfluidics uses channels filled with oil to hold separated aqueous droplets (similar to an emulsion). Within these droplets, single cells can be contained and thus be isolated (Figure 5a). Droplet-based microfluidic concepts can separate single cells either randomly according to Poisson's distribution [46] (similar to the limiting dilution method discussed above) or with even higher yields of over $80 \%$ [49]. The biggest advantage of droplets-in-oil-based cell separation and sorting technologies in general is the tremendous throughput of up to several thousand single-cells per second [49].

Pneumatic membrane valves use pressurized air to deflect an elastomer membrane. This membrane deflection closes a microfluidic channel below (Figure 5b). This allows for digitally opening or closing channels in a microfluidic network. Valve-based approaches need a cell detection unit or an operator to isolate cells individually. Typically, these systems are limited in throughput, compared to the droplets-in-oil-technology described before.

Hydrodynamic traps are passive structures in a microfluidic channel that allow only one cell to enter the "trap" (Figure 5). Typically, double occupation is minimized by adjusting the trap size to the average cell size in a given sample. Such systems can operate on a large number of cells in parallel by using a large number of traps [48]. The commercial system C1 from Fluidigm Corp. for example, allows for isolation and subsequent genetic analysis, of up to 96 individual cells in parallel. Hydrodynamic trapping can even be integrated into handheld pipettes to enable manual single-cell pipetting [50] without the need of micromanipulation under a microscope.

Furthermore, approaches to miniaturize flow cytometers by use of microfluidic technologies have been proposed [51]. One of the goals of this field of research is to bring the advantages of flow cytometers (see Section 3.1) such as cell sorting and counting to small and affordable devices, which can 
potentially be portable. Microfluidic lab-on-a-chip technologies for single-cell applications demonstrate exciting opportunities, but are mostly specifically designed to serve a particular application and therefore exhibit only little flexibility regarding upstream sample preparation and downstream analysis methods. To increase the flexibility and enable easier interfacing with other upstream and downstream methods, the authors of this review have proposed previously a flexible single-cell isolation system (Figure 6), based on inkjet-like single-cell printing [52,53]. This so called single-cell printer (SCP) uses an imaging system and automated object recognition algorithms to detect cells in a microfluidic dispenser chip that can produce droplets similar to an inkjet printer. Cells are classified in the nozzle of the chip and subsequently ejected within a microdroplet ( $60 \mu \mathrm{m}$ in diameter) to be deposited onto various substrates. Droplets containing no cell or multiple cells are deflected in flight towards a waste container by vacuum suction. The suitability of the SCP for biomedical applications such as single-cell genomics [54] and clonal cell line production has already been demonstrated [53].

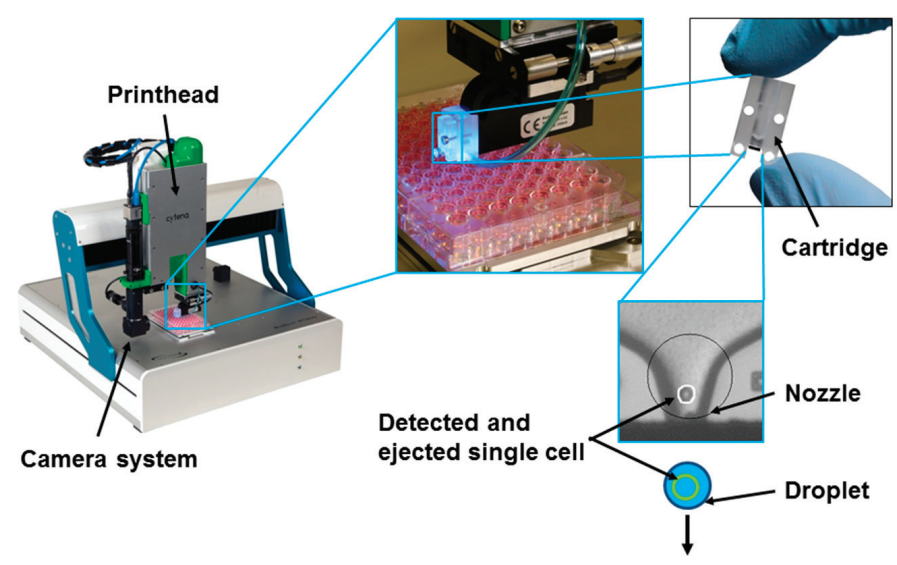

Figure 6. Single-cell printer (SCP) for single-cell isolation. A microfluidic dispenser chip integrated in a polymer cartridge is filled with cell suspension. An automated object recognition algorithm detects cells in the dispenser nozzle prior to the dispensation. This allows for ejection of droplets containing one single-cell only and their deposition in direction of the arrow on various substrates, such as micro-well plates.

\section{Patent Search for Single-Cell Separation Technologies}

In order to identify emerging single-cell separation technologies currently not well known by the scientific community, a patent search has been performed. The reasoning behind this complementary approach is that a market analysis like described above can only be expected to reveal technologies that are sufficiently well known within the user community, while novel technologies that are probably in a pre-commercialization phase are not necessarily known by this group of persons. Still, such emerging technologies could become relevant or even displace existing technologies in the future. In order to screen for novel technologies, we performed a patent search at the European Patent Office (EPO) in the worldwide database for patents. In detail, we were applying the "Smart search" on the EPO 
homepage (http://www.epo.org/ searching/free/espacenet.html) using combinations of search termini based on Boolean operations: $(\mathrm{txt}=$ "single cell" and $(\mathrm{txt}=$ isolation OR txt $=$ separation $))$ NOT $(\mathrm{txt}=$ fuel OR txt = solar). This search led to 179 results, which can be found as a complete list in the supplement (Table S1). The titles and abstracts of the 179 results were carefully reviewed in consideration of their relevance as actual isolation technology for single biological cells. Conversely, the patents considered to be particularly relevant should not represent:

- Only an analysis method of single cells.

- A common method using the term "single cell suspension" without addressing specifically a method for single-cell isolation.

- A cell separation method, which is already established and only part of a patented workflow.

- Other, in this context, irrelevant methods by using the term "cell" in a non-biological context such as for a battery or a chamber in a technical device. Therefore, the terms "fuel" and "solar" were already excluded from the original search from the beginning (see above).

According to these criteria 25 patents were selected from the list of search results based on the information provided in the title and abstract of these patents. The resulting list of relevant patents is presented in Table 2.

Table 2. Patents of single-cell isolation technologies identified in the worldwide database of the European Patent Office (EPO).

\begin{tabular}{|c|c|c|}
\hline Title & EPO Publication Number & Reference \\
\hline $\begin{array}{l}\text { Methods for multiplex analytical } \\
\text { measurements in single cells of solid tissues }\end{array}$ & AU2013315409 (A1) & {$[55]$} \\
\hline Single-cell isolation screen adapted with pipettor tip & CN104195036 (A) & {$[56]$} \\
\hline $\begin{array}{l}\text { An integrated microfluidic device for single-cell } \\
\text { isolation, cell lysis and nucleic acid extraction * }\end{array}$ & CA2817775 (A1) & {$[57]$} \\
\hline System and method for capturing and analyzing cells * & US2014349867 (A1) & {$[58]$} \\
\hline $\begin{array}{c}\text { Single-cell automatic analysis device based on } \\
\text { dual-optical-path micro-fluidic chip * }\end{array}$ & CN203929785 (U) & {$[59]$} \\
\hline $\begin{array}{l}\text { Microfluidic devices and methods for cell sorting, } \\
\text { cell culture and cells based diagnostics and therapeutics * }\end{array}$ & US2014248621 (A1) & {$[60]$} \\
\hline High-throughput single-cell imaging, sorting, and isolation * & US8934700 (B2); US2014247971 (A1) & [61] \\
\hline $\begin{array}{l}\text { Automatic single cell analysis method } \\
\text { based on microfluidic system * }\end{array}$ & CN103926190 (A) & {$[62]$} \\
\hline Apparatus for single cell separation and position fixing * & US2013129578 (A1); US8475730 (B2) & [63] \\
\hline Method and apparatus for single cell isolation and analysis & US2012315639 (A1) & [64] \\
\hline Apparatus for magnetic separation of cells & US2012045828 (A1) & [65] \\
\hline $\begin{array}{l}\text { Method and apparatus for the discretization } \\
\text { and manipulation of sample volumes * }\end{array}$ & CN102187216 (A) & {$[66]$} \\
\hline Plate for separating single cell & JP2011152108 (A); JP5622189 (B2) & [67] \\
\hline
\end{tabular}


Table 2. Cont.

\begin{tabular}{ccc}
\hline Title & EPO Publication Number & Reference \\
\hline $\begin{array}{c}\text { Array apparatus for separation of single cell * } \\
\begin{array}{c}\text { Device and method for continuously analyzing } \\
\text { single-cell contents by miniflow control chip at high speed * }\end{array}\end{array}$ & KR20110037345 (A); KR101252829 (B1) & {$[68]$} \\
\hline $\begin{array}{c}\text { Complete set of equipment for } \\
\text { single cell gel electrophoresis test }\end{array}$ & CN2016623053 (A); CN101923053 (B) & {$[69]$} \\
\hline Single cell analysis of membrane molecules * & US2009173631 (A1) & {$[70]$} \\
\hline $\begin{array}{c}\text { Single-cell inclusion analytical method based } \\
\text { on micro-fluidic chip * }\end{array}$ & CN101393124 (A) & {$[71]$} \\
\hline $\begin{array}{c}\text { Analytical system based on porous material } \\
\text { for highly parallel single cell detection * }\end{array}$ & US2008020453 (A1) & {$[73]$} \\
\hline Single cell isolation apparatus and method of use & US6538810 (B1) & {$[74]$} \\
\hline $\begin{array}{c}\text { Cell isolation and screening device and } \\
\text { method of using same * }\end{array}$ & WO03011451 (A1) & {$[75]$} \\
\hline Cell transfer mechanism and cell fusion apparatus * & JPH0731457 (A) & {$[76]$} \\
\hline $\begin{array}{c}\text { Device for automatically testing single cell dielectric } \\
\text { spectrum based on composite dielectrophoresis }\end{array}$ & CN201075104 (Y) & {$[77]$} \\
\hline High-pass cell separation device and use method therefor * & CN1962845 (A) & {$[78]$} \\
\hline Cell inclusions analysis method based on microfluid chip * & CN1734265 (A) & {$[79]$} \\
\hline
\end{tabular}

*, indicate patents which can be assigned to the field of microfluidics.

Considering that isolation of biological cells means handling of particles in liquid typically in the range of $10-100 \mu \mathrm{m}$ in diameter, the majority of these patents (17 out of 25) can be assigned to the field of microfluidics, as indicated by the appearance of "microfluidic" or terms inherent to microfluidics in the title, abstract and/or original document of the respective patents. Since microfluidics has been described in section 3.5 in general terms, the authors refrain from a full discussion of these technologies. The remaining rather "non-microfluidic" technologies utilize a targeted lysis within a tissue [55], a separation sieve for a conventional pipette tip [56], antibody-conjugated magnetic beads [64,65], adjustable permeable wells on a plate [67], electrophoresis [70], tailor-made wells on a membrane [74], and dielectrophoresis [77] for the isolation of single cells.

\section{Future Potential of Single-Cell Technologies}

There are currently several indicators that suggest that the field of single-cell analysis is going to grow further in the coming years. One of them is the recent acknowledgments of the field by the journal Nature Methods. The increased interest is supported by Nature Methods selecting "single-cell methods" as method to watch 2011 [80] and "single-cell sequencing" as method of the year 2013 [81]. The demand for single-cell handling technologies is expected to grow simultaneously with the field of single-cell analysis.

Figure 7 shows the expected increase in importance of single-cell technologies as seen by the participants of the market study, described in Section 2. In the online survey participants were asked to rank the importance of single-cell research from not important to very important to their present as well as for their future work. For the present year (2014) the answer with the highest score (mode 
of the data set) was fairly important. For 2017 the expectation with the highest score was very important (the highest possible score). These data were acquired amongst German researchers. A similar trend is expected to hold for worldwide researchers, which is in accordance to market data published [6].

Furthermore, participants, who did not have any experience with single-cell technologies in 2014, were asked about their future plans. Out of these, $79 \%$ planned to work with single-cell technologies within the next five years. $48 \%$ even planned to start working with single-cell technologies in 2015 .

In recent years, the field of single-cell analysis has seen a few commercial products hit the market and more are expected to come. Applications currently envisioned or implemented include next generation sequencing (NGS) of single cells [82], isolation of circulating tumor cells for diagnostic purposes [83], or single-cell proteomics [84].

\section{Importance of single-cell research}

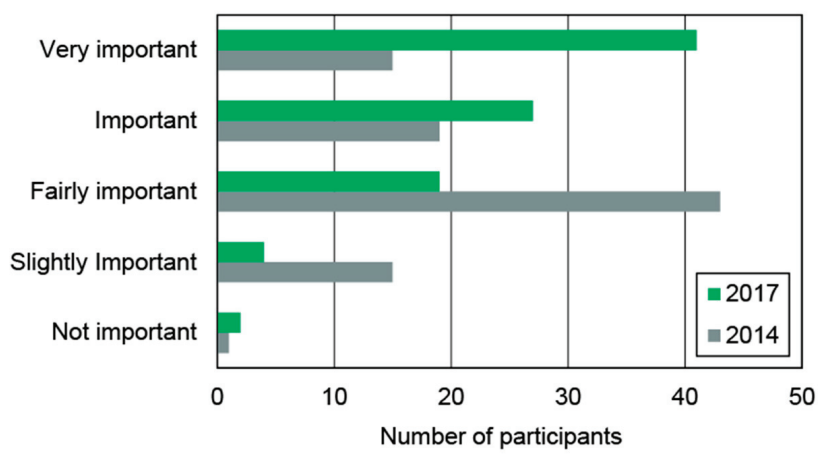

Figure 7. Importance of single-cell analysis to German cell researchers in 2014 and estimated for 2017. This data was derived by a survey amongst 210 participants from German universities, research institutes and industry. A strong growth of interest is expected over the next years.

\section{Discussion}

The requirements for technologies to separate and isolate single cells from samples of different nature are as heterogeneous as the purpose for which the cells are used downstream of the separation and isolation process. Depending on the specific requirements, some of the mentioned technologies might be more suitable than others to enable a specific single cell application. In the following, strengths as well as shortcomings of the reviewed commercial technologies are listed and compared to these requirements qualitatively. However, quantitative performance parameters for the various technologies cannot be determined, since this would require focusing on a specific application, which is out of scope of this article. The actual result of a single-cell isolation process - especially with respect to efficiency and cell viability - is depending on many factors like cell type, sample preparation, device calibration, sorting mode, substrate, and many more factors that are hard to quantify in general (e.g., operator skill for manual methods). Thus, the assessment of the different 
technologies has to remain more general and instead general properties of the technologies like throughput or cell viability, etc. are discussed.

\subsection{General Aspects}

Defining general requirements in the field of single-cell handling and analysis is not trivial due to the heterogeneity of the applications mentioned above. The following non-exhaustive list seeks to cover general requirements that many applications have in common.

Sample nature and origin defines to a large extent which technologies can be used at all. The information to be determined from the cells of interest could largely differ: in solid samples often tissue architecture or cell-cell interactions are of interest, while in cell suspensions heterogeneity studies of the cell population are mostly the primary objective.

Cell integrity is often required throughout the cell isolation process. Especially when the genome or proteome is the target of the analysis, cell integrity should be kept prior to lysis to avoid early degradation of DNA/RNA.

Cell viability is required when isolating single-cells for the purpose of production of monoclonal cell cultures or for studying stem cell differentiation. Cells respond to stress factors like mechanical forces, radiation, chemical changes in their environment, etc. which can lead to differentiation, reduced viability or even apoptosis. Technologies should provide sufficiently "gentle" extraction and handling when operating on living cells.

Throughput in terms of single cells isolated per second as well as the targeted total number of single cells is another important factor. Especially when large populations with low abundance of target cells are given (e.g., for CTC (Circulating tumor cells) applications), high throughput or high parallelization is mandatory and manual procedures are prohibitive.

Rare samples with a low amount of cells require technologies capable of dealing with small sample volumes and providing low dead volumes. In this context the separation yield (see below) is often a key issue as well in this context to prevent the loss of cells of interest.

Purity of the isolated single cells is crucial when analyzing cellular DNA and RNA. Isolating the cell of interest and while excluding any other contamination from the liquid suspension (e.g., cell fragments, free-DNA, etc.) is of highest importance. Transfer volume (the droplet or pipetting volume the cell is typically enclosed in during transfer to the target) and cross-contamination come into play here as well, as they determine the amount of contaminants in the aliquot containing the single cell.

Efficiency concerning yield of the single-cell isolation process can be of importance when using homogeneous samples containing a large number of cells (e.g., for cloning). However, it's a lot more important when performing single-cell analysis on a rare cell sample or when using complex and costly reagents. Besides the costs per cell that come into play from the economical point of view (e.g., for amplification and library preparation for next-generation sequencing [85]), the analysis of individually selected cells rather than randomly seeded ones or the analysis of a complete population (100\% analysis) can impose strict requirements on the separation efficiency. Preventing aliquots that are empty or occupied with multiple cells can be mandatory in certain applications while in others it might be more important that no cell must be lost in the isolation process. 
Besides these most relevant general criteria for the assessment of different technologies each application might impose additional specific requirements that have to be carefully considered. For applications with living cells, for example, often carry over-free and sterile operation conditions are requested which calls for technologies relying on disposable components (e.g., microfluidic chips).

Obviously, each single cell separation technology exhibits specific features with respect to the above mentioned aspects that have to be matched to the application under consideration. Usually, this problem is addressed from the side of the application (e.g., single-cell analysis, monoclonal cell cultures, etc.) for which the requirements are usually well known. In the following the view point should be shifted towards considering the specific features of the previously discussed technologies.

\subsection{Flow Cytometry}

FACS systems provide high throughput in terms of single-cell analysis and sorting. Paired with high flexibility in terms of cell type, standardized substrates, and sorting modes FACS is a powerful tool. Moreover its suitability for rare cell sorting (subpopulations $<1 \%$ ) has increasing diagnostic prospect when analyzing heterogeneous cell samples. Some of the FACS systems are able to deposit single cells in micro-well plates with high purity and yield within the time frame of minutes to enable further downstream analysis such as NGS (Next generation sequencing). The popularity and wide-spread use of FACS systems makes them accessible to a broad range of users.

Nevertheless, for certain applications FACS systems are still limited to some extent. Cells must be in suspension meaning tissues need to be dissociated resulting in loss of cellular functions and cell-cell interactions as well as tissue architecture [86]. Subpopulations with similar marker expression are difficult to differentiate and overlap of emission spectra between fluorochromes may lead to an increasing noise level making low-intensity samples unavailable for detection. Further, FACS sorting may have non-negligible effects on cell viability, which was demonstrated for Chinese Hamster Ovary (CHO) cells and for a human monocytic cell line (THP1) by trypan blue exclusion and necrosis/apoptosis assays [87].

Minimal sample volume for FACS systems is in the range of several hundreds of microliters to milliliters. This is due to the fact, that typically long sections of tubing cause high dead volumes, preventing the use of rare samples, especially when the entire sample needs to be analyzed. And finally, sterile operation is difficult to achieve by FACS systems in general due to the complex system consisting of non-disposable components. However, such limitations are not always present or significant, but strongly dependent on instrument, process parameters (e.g., speed, laser type, etc.), cell type, and application.

\subsection{Laser Capture Microdissection}

Whenever single cells need to be isolated from solid samples (e.g., tissue, biopsies) LCM systems are the commonly applied tool of choice. For such applications a single cell's location within the tissue architecture is essential to know. LCM systems enable separation and isolation of individual cells for downstream analysis. Based on optical microscopes coupled with a coaxial cutting laser and computer assisted control, such systems are relatively easy to handle. The sharply focused pulse 
lasers cut with sub-micrometer precision and without introducing deleterious heat to the tissue. Another benefit might be that operators actually decide on every cell to select and isolate rather than leaving the decision to automated systems or statistical distributions.

Although modern LCM systems provide a higher level of user-friendliness and automation, the selection and isolation process remains operator based and therefore strongly limits the throughput. The integrity of extracted cells is important for reliable downstream analysis of biomolecules such as DNA, RNA, and proteins [88]. Depending on the quality of fixation [32] and cell extraction method used (adhesive tape, gravity, catapulting) single-cell integrity might be compromised [89]. It might remain unclear, if the cell was actually transferred and/or if any contaminants (e.g., fragments of adjacent cells) were transferred to the substrate along with the cell of interest, especially when using contact-based cell extraction (adhesive cap) [90].

\subsection{Micromanipulator}

Micromanipulators are microscope assisted picking tools allowing for targeted isolation of individual single cells from suspensions, which is a feature not shared by many other technologies. The operator selects the cells to be isolated and performs the aspiration, transfer, and dispensation. Similar to LCM systems, the targeted isolation of a specific cell under microscope vision is one of the key benefits of this technology.

Although being a very flexible technology in terms of cell types and substrates, the serial and manual process of obtaining single cells limits the overall throughput. Furthermore, with the majority of the systems it is not possible to observe and control the correct transfer of the single-cell to its target location. Once the micropipette leaves the microscope's optical focus plane the transfer volume containing the cell is unobservable as is the actual transition to the target. To actually confirm if a single-cell has successfully been transferred additional observation of the target is required. Recent approaches target improvement on this towards fully automated isolation and placement of single cells assisted by video systems and image processing algorithms [91].

\subsection{Limiting Dilution}

In many pharmaceutical companies, fully automated pipetting robots perform limiting dilution in great numbers at considerable throughput. The process is simple, reproducible and to a certain extent cost-efficient since the degree of automation is very high. However, due to the statistical nature of the process and the lack of control over an individual cell, often further technologies are required downstream to prove the presence of single cells in a specific well, such as automatic microscopic imaging systems. However, limiting dilution remains a simple, gentle, and relatively cost efficient process to obtain single cells with reasonable throughput, but lacking the controlled isolation and sorting as well as proof of single-cell presence. Combined with upstream sorting or enrichment techniques it can constitute an appropriate tool to easily separate viable single cells for downstream analysis. 


\subsection{Microfluidics}

Microfluidics plays an increasing role in establishing entire workflows for single-cell separation, isolation, and analysis. Although the number of commercially available systems is still low, this field of research itself is highly dynamic. Microfluidic systems can be operated with very low volumes regarding cell sample as well as reagents, which is advantageous for rare cell applications as well as from an economical point of view. Developed as disposables and once produced with mass-fabrication techniques those systems could provide an attractive alternative, especially with regard to clinical applications: when using disposables, cross-contamination between subsequent samples is not an issue.

Recent hurdles preventing a broader market-entry of microfluidic technologies might be the low degree of flexibility offered by a specific microfluidic chip. Microfluidic systems-unlike the more established technologies discussed before - are often restricted to one single application (e.g., genomic single-cell analysis). Regarding the complexity and variety of single-cell applications throughout the field, and in view of continuously newly established analysis methods, this likely is one of the greatest drawbacks of microfluidic technologies for research applications. Leading scientists and microfluidic companies often regret the absence of a "killer application" supporting their technology (This is true for the entire microfluidic field, not only for those devices targeting single-cell applications.) to enter the markets [92-94]. Still, when a sufficiently standardized workflow of isolation, sorting, and analysis can be established by a microfluidic approach, there are probably few other technologies that can provide similar performance in that specific case.

\subsection{Patents and Emerging Technologies}

The patent search performed for this review revealed that $68 \%$ (17 out of 25$)$ of the related patents cover microfluidic devices. This patent trend has been present since the middle of the 90s [95] and emphasizes the strong driving forces generated by research and emerging industries. Besides microfluidics few patents deal with alternative technologies e.g., separation by dielectrophoresis or size/affinity based filtering. Partially such alternative technologies are also combined with microfluidic approaches at chip level. Whether or not such special technologies will become relevant in the future depends to a large degree on the applications that can be enabled by them. As outlined before, a strong match between application requirements and technology features can render any technology relevant in the context of a well matching application.

\section{Conclusions}

The review focused on the most frequently used single-cell isolation technologies as derived from a market survey amongst German scientists. It can be concluded that there is no universal technology suitable for all single-cell applications. However, the vast majority of applications can be addressed by at least one of the technologies available today.

Regarding performance FACS systems have the main benefit of high throughput and sorting capability but are cost-intensive and potentially harmful to some cells. LCM is ideal for isolation of single cells from solid tissue and quite unique in this regard. Micromanipulator assisted cell picking 
is a manual process and therefore slow, but provides maximum control over individual cells. Limiting dilution relies on statistical distribution, is simple to implement and can be automated. However, the presence of single cells often needs to be verified subsequently. Apart from these established technologies, microfluidic technologies allow for integration of entire application specific workflows and only require small amounts of sample and reagents. Thus, microfluidic technologies have potential but are still lacking broad commercial presence, probably due to a lower degree of flexibility. To summarize the topic, Table 3 provides an overview about the key features of the presented technologies.

The worldwide patent search carried out with the objective to identify emerging technologies, resulted in 179 patents out of which 25 have been identified to be particularly relevant to the field. 17 out of those 25 are related to the field of microfluidics, again emphasizing the importance of this evolving field. From the market study it can also be concluded, that the field of single-cell analysis can be expected to grow significantly in the following years and the need for single-cell isolation technologies is likely to increase simultaneously. Presumably, established commercial technologies as well as novel microfluidic devices will contribute equally to advances in this field in the coming years.

Table 3. Selected features of discussed technologies. Rating based on the authors' personal experience and knowledge.

\begin{tabular}{|c|c|c|c|c|c|}
\hline Technology & Automation Level & Throughput & $\begin{array}{c}\text { Impact on } \\
\text { Cell Integrity }\end{array}$ & $\begin{array}{c}\text { Control over } \\
\text { Individual Cell } \\
1\end{array}$ & $\begin{array}{c}\text { Compatibility with } \\
\text { Established Workflows } \\
2\end{array}$ \\
\hline $\begin{array}{l}\text { Fluorescence-Activated } \\
\text { Cell Sorting (FACS) }\end{array}$ & Automatic & High & $\begin{array}{c}\text { Often } \\
\text { impairing }\end{array}$ & Yes & High \\
\hline Limiting dilution & Manual or automatic & Moderate & Gentle & No & High \\
\hline Micromanipulation & Manual & Low & Moderate & Yes & Moderate \\
\hline Laser-capture microdissection & Manual & Low & $\begin{array}{c}\text { Often } \\
\text { impairing }\end{array}$ & Yes & Low \\
\hline Microfluidics (Lab-on-a-Chip) & Automatic & Low to high & Diverse & Typically not & Low \\
\hline Microfluidics (inkjet-like printing) & Automatic & Moderate & Gentle & Yes & High \\
\hline
\end{tabular}

${ }^{1}$, Possibility for active selection of single cells before their isolation in contrast to random distribution of individual cells; ${ }^{2}$, Compatibility with commercially available substrates such as microtiter plates, tubes, slides, etc.

\section{Supplementary Materials}

Supplementary materials can be found at http://www.mdpi.com/1422-0067/16/08/16897/s1.

\section{Acknowledgments}

We gratefully acknowledge financial support by EU FP7 project SICTEC (GA 606167) and national BMWi/ESF funded project SCOD (FKZ 03EFBBW057). Further we would like to thank the University of Mannheim, Germany for their support in conducting the German market study and HTStec, UK for cooperation regarding their international market study. 


\section{Author Contributions}

The authors contributed as follows: Andre Gross, writing chapters 3.1-3.4, 6, Figure 4; Jonas Schoendube, writing chapters 2, 3.5, 5, Figures 1,2,5,6,7; Stefan Zimmermann, patent search, writing chapters 1, 4, Figure 3; Maximilian Steeb executed the survey for the German market study; Peter Koltay, writing chapter 7, editing and proof-reading; Roland Zengerle, design of the study, editing and proof-reading. This review presents only major technologies and techniques and does not claim to be comprehensive and complete in all fields. Technology ratings like in Table 3 are personal opinions of the authors based on their experience in the field.

\section{Conflicts of Interest}

The authors Jonas Schoendube, Andre Gross, Peter Koltay, and Roland Zengerle have equity interests in cytena $\mathrm{GmbH}$, a company that is developing single-cell analysis and handling solutions based on the cell printing technology described in Section 3.5.

\section{References}

1. Blainey, P.C.; Quake, S.R. Dissecting genomic diversity, one cell at a time. Nat. Methods. 2014, 11, 19-21.

2. Van Loo, P.; Voet, T. Single cell analysis of cancer genomes. Curr. Opin. Genet. Dev. 2014, 24, 82-91.

3. Ding, L.; Wendl, M.C.; McMichael, J.F.; Raphael, B.J. Expanding the computational toolbox for mining cancer genomes. Nat. Rev. Genet. 2014, 15, 556-570.

4. Ilie, M.; Hofman, V.; Long, E.; Bordone, O.; Selva, E.; Washetine, K.; Marquette, C.H.; Hofman, P. Current challenges for detection of circulating tumor cells and cell-free circulating nucleic acids, and their characterization in non-small cell lung carcinoma patients. What is the best blood substrate for personalized medicine? Ann. Transl. Med. 2014, 2, 107.

5. BIOCOM AG. Biotechnology Database. Available online: http://www.biotechnologie.de/BIO/ Navigation/EN/Databases/biotechnology-db.html (accessed on 30 June 2015).

6. HTStec. Single Cell Technologies Trends 2014; Available online: http://selectbiosciences.com/ MarketReportsID.aspx?reportID=83 (accessed on 30 June 2015).

7. Moldavan, A. Photo-Electric Technique for the Counting of Microscopial Cells; Science: New York, NY, USA, 1934; Volume 80, pp. 188-189.

8. Gucker, F.T.; O'Konski, C.T. A photoelectronic counter for colloidal particles. J. Am. Chem. Soc. 1947, 69, 2422-2431.

9. Coulter, W.H. Means for Counting Particles Suspended in a Fluid. U.S. Patent 2,656,508 A, 20 October 1953.

10. Fulwyler, M.J. Electronic separation of biological cells by volume. Science 1965, 150, 910-911.

11. Fulwyler, M.J. Particle Separator. U.S. Patent 3,380,584 A, 30 April 1968.

12. Dittrich, W.; Goehde, W. Automatisches Mess- und Zaehlgeraet fuer die Teilchen einer Dispersion. D.E. Patent 1,815,352 A1, 14 January 1971.

13. Shapiro, H.M. Practical Flow Cytometry, 4th ed.; Wiley-Liss: New York, NY, USA, 2003. 
14. Kohler, G.; Milstein, C. Continuous cultures of fused cells secreting antibody of predefined specificity. Nature 1975, 256, 495-497.

15. Herzenberg, L.A.; Parks, D.; Sahaf, B.; Perez, O.; Roederer, M.; Herzenberg, L.A. The history and future of the fluorescence activated cell sorter and flow cytometry: A view from Stanford. Clin. Chem. 2002, 48, 1819-1827.

16. Underwood, A.P.; Bean, P.A. Hazards of the limiting-dilution method of cloning hybridomas. J. Immunol. Methods 1988, 107, 119-128.

17. Brown, M.; Wittwer, C. Flow cytometry: Principles and clinical applications in hematology. Clin. Chem. 2000, 46, 1221-1229.

18. Valet, G. Past and present concepts in flow cytometry: A European perspective. J. Biol. Regul. Homeost. Agents 2003, 17, 213-222.

19. Davey, H.M.; Kell, D.B. Flow cytometry and cell sorting of heterogeneous microbial populations: The importance of single-cell analyses. Microbiol. Rev. 1996, 60, 641-696.

20. Lacombe, F.; Belloc, F. Flow cytometry study of cell cycle, apoptosis and drug resistance in acute leukemia. Hematol. Cell Ther. 1996, 38, 495-504.

21. McCoy, J.P.; Carey, J.L. Recent advances in flow cytometric techniques for cancer detection and prognosis. Immunol. Ser. 1990, 53, 171-187.

22. Emmert-Buck, M.R.; Bonner, R.F.; Smith, P.D.; Chuaqui, R.F.; Zhuang, Z.; Goldstein, S.R.; Weiss, R.A.; Liotta, L.A. Laser Capture microdissection. Science 1996, 274, 998-1001.

23. Espina, V.; Heiby, M.; Pierobon, M.; Liotta, L.A. Laser capture microdissection technology. Expert Rev. Mol. Diagn. 2007, 7, 647-657.

24. Vogel, A.; Noack, J.; Hüttman, G.; Paltauf, G. Mechanisms of femtosecond laser nanosurgery of cells and tissues. Appl. Phys. B 2005, 81, 1015-1047.

25. Vogel, A.; Lorenz, K.; Horneffer, V.; Hüttmann, G.; von Smolinski, D.; Gebert, A. Mechanisms of laser-induced dissection and transport of histologic specimens. Biophys. J. 2007, 93, 4481-4500.

26. Esposito, G. Complementary techniques: Laser capture microdissection-increasing specificity of gene expression profiling of cancer specimens. Adv. Exp. Med. Boil. 2007, 593, 54-65.

27. Fink, L.; Kwapiszewska, G.; Wilhelm, J.; Bohle, R.M. Laser-microdissection for cell type- and compartment-specific analyses on genomic and proteomic level. Exp. Toxicol. Pathol. 2006, 5, $25-29$.

28. Fink, L.; Bohle, R.M. Laser microdissection and RNA analysis. Methods Mol. Biol. 2005, 293, 167-185.

29. Nakamura, N.; Ruebel, K.; Jin, L.; Qian, X.; Zhang, H.; Lloyd, R.V. Laser capture microdissection for analysis of single cells. Methods Mol. Med. 2007, 132, 11-18.

30. Keays, K.M.; Owens, G.P.; Ritchie, A.M.; Gilden, D.H.; Burgoon, M.P. Laser capture microdissection and single-cell RT-PCR without RNA purification. J. Immunol. Methods 2005, 302, 90-98.

31. Vandewoestyne, M.; Deforce D. Laser capture microdissection in forensic research: A review. Int. J. Leg. Med. 2010, 124, 513-521. 
32. DeCarlo, K.; Emley, A.; Dadzie, O.E.; Mahalingam, M. Laser capture microdissection: Methods and applications. Methods Mol. Biol. 2011, 755, 1-15.

33. Lefkovits, I.; Pernis, B. Immunological Methods; Elsevier Science: Burlington, MA, USA, 1979.

34. Goding, J.W. Antibody production by hybridomas. J. Immunol. Methods 1980, 285-308.

35. Fuller, S.A.; Takahashi, M.; Hurrell, J.G. Cloning of hybridoma cell lines by limiting dilution. Curr. Protoc. Mol. Biol. 2001, doi:10.1002/0471142727.mb1108s01.

36. Yokoyama, W.M.; Christensen, M.; Dos Santos, G.; Miller, D.; Ho, J.; Wu, T.; Dziegelewski, M.; Neethling, F.A. Production of monoclonal antibodies. Curr. Protoc. Immunol. 2013, doi:10.1002/0471142735.im0205s 102 .

37. Staszewski, R. Cloning by limiting dilution: An improved estimate that an interesting culture is monoclonal. Yale J. Biol. Med. 1984, 57, 865-868.

38. Smith, R. Cell technology for cell products. Proceedings of the 19th ESACT Meeting, Harrogate, UK, 5-8 June 2005; Springer: Dordrecht, The Netherlands, 2007.

39. Fröhlich, J.; König, H. New techniques for isolation of single prokaryotic cells. FEMS Microbiol. Rev. 2000, 24, 567-572.

40. Brehm-stecher, B.F.; Johnson, E.A. Single-cell microbiology: Tools, technologies, and applications. Microbiol. Mol. Biol. Rev. 2004, 68, 538-559.

41. Wright, G.; Tucker, M.J.; Morton, P.C.; Sweitzer-Yoder, C.L.; Smith, S.E. Micromanipulation in assisted reproduction: A review of current technology. Curr. Opin. Obstet. Gynecol. 1998, 10, 221-226.

42. Li, C.-X.; Wang, G.-Q.; Li, W.-S.; Huang, J.-P.; Ji, A.-Q.; Hu, L. New cell separation technique for the isolation and analysis of cells from biological mixtures in forensic caseworks. Croat. Med. J. 2011, 52, 293-298.

43. Sims, C.E.; Allbritton, N.L. Analysis of single mammalian cells on-chip. Lab Chip 2007, 7, $423-440$.

44. Lecault, V.; White, A.K.; Singhal, A.; Hansen, C.L. Microfluidic single cell analysis: From promise to practice. Curr. Opin. Chem. Biol. 2012, 16, 381-390.

45. Gossett, D.R.; Weaver, W.M.; Mach, A.J.; Hur, S.C.; Tse, H.T.K.; Lee, W.; Amini, H.; di Carlo, D. Label-free cell separation and sorting in microfluidic systems. Anal. Bioanal. Chem. 2010, 397, 3249-3267.

46. Brouzes, E.; Medkova, M.; Savenelli, N.; Marran, D.; Twardowski, M.; Hutchison, J.B.; Rothberg, J.M.; Link, D.R.; Perrimon, N.; Samuels, M.L. Droplet microfluidic technology for single-cell high-throughput screening. Proc. Natl. Acad. Sci. USA 2009, 106, 14195-14200.

47. Gomez-Sjoberg, R.; Leyrat, A.A.; Pirone, D.M.; Chen, C.S.; Quake, S.R. Versatile, fully automated, microfluidic cell culture system. Anal.Chem. 2007, 79, 8557-8563.

48. Di Carlo, D.; Wu, L.Y.; Lee, L.P. Dynamic single cell culture array. Lab Chip 2006, 6, 1445-1449.

49. Edd, J.F.; di Carlo, D.; Humphry, K.J.; KÇôster, S.; Irimia, D.; Weitz, D.; Toner, M. Controlled encapsulation of single-cells into monodisperse picolitre drops. Lab Chip 2008, 8, 1262-1264.

50. Zhang, K.; Han, X.; Li, Y.; Li, S.Y.; Zu, Y.; Wang, Z.; Qin, L. Hand-held and integrated single-cell pipettes. J. Am. Chem. Soc. 2014, 136, 10858-10861. 
51. Ateya, D.A.; Erickson, J.S.; Howell, P.B.; Hilliard, L.R.; Golden, J.P.; Ligler, F.S. The good, the bad, and the tiny: A review of microflow cytometry. Anal. Bioanal. Chem 2008, 391, $1485-1498$.

52. Yusof, A.; Keegan, H.; Spillane, C.D.; Sheils, O.M.; Martin, C.M.; O’Leary, J.J.; Zengerle, R.; Koltay, P. Inkjet-like printing of single-cells. Lab Chip 2011, 11, 2447-2454.

53. Gross, A.; Schondube, J.; Niekrawitz, S.; Streule, W.; Riegger, L.; Zengerle, R.; Koltay, P. Single-cell printer: Automated, on demand, and label free. J. Lab. Autom. 2013, 18, 504-518.

54. Stumpf, F.; Schoendube, J.; Gross, A.; Rath, C.; Niekrawietz, S.; Koltay, P.; Roth, G. Single-cell PCR of genomic DNA enabled by automated single-cell printing for cell isolation. Biosens. Bioelectron. 2015, 69, 301-306.

55. Finski, A.; Macbeath, G. Methods for Multiplex Analytical Measurements in Single Cells of Solid Tissues. Patent AU2013315409 (A1), 2 April 2015.

56. Liu, D. Single-Cell Isolation Screen Adapted with Pipettor Tip. Patent CN104195036 (A), 10 December 2014.

57. Yao, C.D. An Integrated Microfluidic Device for Single-Cell Isolation, Cell Lysis and Nucleic Acid Extraction. Patent CA2817775 (A1), 29 November 2014.

58. Handique, K.; Gogoi, P.; Javdani, S.S.; Zhou, Y. System and Method for Capturing and Analyzing Cells. US2014349867 (A1), 27 November 2014.

59. Liu, Y.; Li, J.; Chen, Y.; Jian, D.; Gao, J. Single-Cell Automatic Analysis Device Based on Dual-Optical-Path Micro-Fluidic Chip. Patent CN203929785 (U), 5 November 2014.

60. Collins, J. Microfluidic Devices and Methods for Cell Sorting, Cell Culture and Cells Based Diagnostics and Therapeutics. Patent US2014248621 (A1), 4 September 2014.

61. Bharadwaj, R.; Fathollahi, B. High-Throughput Single-Cell Imaging, Sorting, and Isolation. Patent US8934700 (B2), US2014247971 (A1), 4 September 2014.

62. Liu, Y.; Ban Qing, G.J. Automatic Single Cell Analysis Method Based on Microfluidic System. Patent CN103926190 (A), 16 July 2014.

63. Jeong, O.C. Apparatus for Single Cell Separation and Position Fixing. Patent US2013129578 (A1), US8475730 (B2), 23 May 2013.

64. Deng, G.Y.; Zhang, J.; Tian, F. Method and Apparatus for Single Cell Isolation and Analysis. Patent US2012315639 (A1), 13 December 2012.

65. Davis, R.W.; Jeffrey, S.S.; Mindrinos, M.N.; Pease, R.F.; Powell, A.A.; Talasaz, A.H. Apparatus for Magnetic Separation of Cells. Patent US2012045828 (A1), 23 February 2012.

66. Chiu, D.T.; Cohen, D.E.; Jeffries, G.D.M. Method and Apparatus for the Discretization and Manipulation of Sample Volumes. Patent CN102187216 (A), 14 September 2011.

67. Matsutani, A.; Takada, A. Plate for Separating Single Cell. Patent JP2011152108 (A), JP5622189 (B2), 11 August 2011.

68. Park, J.W.; Jung, M.Y.; Park, S.H. Array Apparatus for Separation of Single Cell. Patent KR20110037345 (A), KR101252829 (B1), 13 April 2011.

69. Yin, X.F.; Xu, C.X.; Liu, J.H. Device and Method for Continuously Analyzing Single-Cell Contents by Miniflow Control Chip at High Speed. Patent CN101923053 (A), CN101923053 (B), 22 December 2010. 
70. Zhang, C.K.; Shi, J.Y.; Zeng, Y.C.; Shen, C.F.; Chen, Y,X.; Chen, L. Complete Set of Equipment for Single Cell Gel Electrophoresis Test. Patent CN201662556(U), 1 December 2010.

71. Boone, T.; Singh, S. Single Cell Analysis of Membrane Molecules. Patent US2009173631 (A1), 9 July 2009.

72. Wu, D.P.; Yu, L.F.; Lin, B.C.; Qin, J.H. Single-Cell Inclusion Analytical Method Based on Micro-Fluidic Chip. Patent CN101393124 (A), 25 March 2009.

73. Ehben, T.; Zilch, C. Analytical System Based on Porous Material for Highly Parallel Single Cell Detection. Patent US2008020453 (A1), 24 January 2008.

74. Karanfilov, C. Single Cell Isolation Apparatus and Method of Use. Patent US6538810 (B1), 25 March 2003.

75. Wang, E.; Kim, E.; Campbell, S.; Kirk, G.L.; Casagrande, R. Cell Isolation and Screening Device and Method of Using Same. Patent WO03011451 (A1), 13 February 2003.

76. Suzuki, H.; Yasunaka, T. Cell Transfer Mechanism and Cell Fusion Apparatus. Patent JPH0731457 (A), 3 February 1995.

77. Ni, Z.H.; Song, C.F.; Yi, H.; Zhu, S.C. Device for Automatically Testing Single Cell Dielectric Spectrum Based on Composite Dielectrophoresis. Patent CN201075104 (Y), 18 June 2008.

78. Shen, B.Y. High-Pass Cell Separation Device and Use Method Therefor. Patent CN1962845 (A), 16 May 2007.

79. Lin, B.Y. Cell Inclusions Analysis Method Based on Microfluid Chip. Patent CN1734265 (A), 15 February 2006.

80. De Souza, N. Single-cell methods. Nat. Methods 2011, 9, 35.

81. Editorial. Method of the Year 2013. Nat. Methods 2013, 11, 1.

82. Shapiro, E.; Biezuner, T.; Linnarsson, S. Single-cell sequencing-based technologies will revolutionize whole-organism science. Nat. Rev. Genet. 2013, 14, 618-630.

83. Yu, M.; Stott, S.; Toner, M.; Maheswaran, S.; Haber, D.A. Circulating tumor cells: Approaches to isolation and characterization. J. Cell Biol. 2011, 192, 373-382.

84. Wu, M.; Singh, A.K. Single-cell protein analysis. Curr. Opin. Biotechnol. 2012, 23, 83-88.

85. Mardis, E.R. The impact of next-generation sequencing technology on genetics. Trends Genet. 2008, 24, 133-141.

86. Jahan-Tigh, R.R.; Ryan, C.; Obermoser, G.; Schwarzenberger, K. Flow cytometry. J. Investig. Dermatol. 2012, 132, doi:10.1038/jid.2012.282.

87. Mollet, M.; Godoy-Silva, R.; Berdugo, C.; Chalmers, J.J. Computer simulations of the energy dissipation rate in a fluorescence-activated cell sorter: Implications to cells. Biotechnol. Bioeng. 2008, 100, 260-272.

88. Bevilacqua, C.; Makhzami, S.; Helbling, J.-C.; Defrenaix, P.; Martin, P. Maintaining RNA integrity in a homogeneous population of mammary epithelial cells isolated by Laser Capture Microdissection. BMC Cell Biol. 2010, 11, 95.

89. Liu A. Laser capture microdissection in the tissue biorepository. J. Biomol. Tech. 2010, 21, 120-125.

90. Fend, F. Laser capture microdissection in pathology. J. Clin. Pathol. 2000, 53, 666-672. 
91. Lu, Z.; Moraes, C.; Zhao, Y.; You, L.D.; Simmons, C.A.; Sun, Y. A micromanipulation system for single cell deposition. In Proceeding of 2010 IEEE International Conference on Robotics and Automation (ICRA 2010), Anchorage, Alaska, 3-8 May 2010; IEEE: New York, NY, USA, May 2010; pp. 494-499.

92. Blow, N. Microfluidics: In search of a killer application. Nat. Methods 2007, 4, 665-668.

93. Whitesides, G.M. The origins and the future of microfluidics. Nature 2006, 442, 368-373.

94. Becker, H. Hype, hope and hubris: The quest for the killer application in microfluidics. Lab Chip 2009, 9, 2119-2122.

95. Haber, C. Microfluidics in commercial applications; an industry perspective. Lab Chip 2006, 6, 1118-1121. 


\title{
Single-Cell Isolation and Gene Analysis: Pitfalls and Possibilities
}

\section{Kjetil Hodne and Finn-Arne Weltzien}

\begin{abstract}
During the last two decades single-cell analysis (SCA) has revealed extensive phenotypic differences within homogenous cell populations. These phenotypic differences are reflected in the stochastic nature of gene regulation, which is often masked by qualitatively and quantitatively averaging in whole tissue analyses. The ability to isolate transcripts and investigate how genes are regulated at the single cell level requires highly sensitive and refined methods. This paper reviews different strategies currently used for SCA, including harvesting, reverse transcription, and amplification of the RNA, followed by methods for transcript quantification. The review provides the historical background to SCA, discusses limitations, and current and future possibilities in this exciting field of research.
\end{abstract}

Reprinted from Int. J. Mol. Sci. Cite as: Hodne, K.; Weltzien, F.-A. Single-Cell Isolation and Gene Analysis: Pitfalls and Possibilities. Int. J. Mol. Sci. 2015, 16, 26832-26849.

\section{Introduction}

Genes are regulated at the single cell level, and the stochastic nature of genes turning on and off results in a temporally heterogeneous gene expression, even within homogenous cell populations [1-9]. This unique feature is often concealed behind average quantification in whole tissues. Based on earlier discoveries of gene expression dynamics, along with recent improvements in robust and sensitive methods, interest in single-cell omics is growing rapidly. In 2013 single-cell sequencing was awarded "method of the year" by Nature Methods [10] demonstrating groundbreaking discoveries and exciting potential in cell biology [11-15]. Today, novel technologies, like lab-on-a-chip, have facilitated large-scale screenings of transcripts within single-cells. This review opens with a historical perspective focusing on nucleic acid amplification and single-cell gene analysis. We then move on to discuss pros and cons regarding different strategies for harvesting and isolation of nucleic acids, and quantification of gene expression, and finally provide some thoughts on future possibilities within the field of single-cell gene expression.

\section{Historical Background-Nucleic Acid Amplification}

The idea of isolating and analyzing small levels of nucleic acids goes back almost five decades. During the work of unraveling the genetic code, working as a researcher in Har Gobind Khorana's laboratory, Kjell Kleppe described for the first time a method for primer-defined enzymatic replication of short DNA fragments. However, at that time little focus was put into Kleppe and Khorana's vision in which a system could target and amplify a specific DNA sequence defined by complementary primers [16]. In fact, it took another decade until Kary Mullis reintroduced the concept of primer-dependent DNA amplification, which we now know as PCR [17]. Through several studies, and subsequent publications, Mullis and Saiki reintroduced and refined Kleppe's 
ideas and described the basic principles of exponential DNA amplification employing two complementary primers for each DNA strand [18,19]. The initial PCR protocol consisted of 20-27 cycles with $2 \mathrm{~min}$ at $95{ }^{\circ} \mathrm{C}$ to separate the two DNA strands followed by 2 min at $37{ }^{\circ} \mathrm{C}$, allowing the primers to anneal and the polymerase to synthesize the complementary strand. However, because of the thermolability of the polymerase (Klenow fragment of Escherichia coli DNA polymerase (I)), it was inactivated during the $95{ }^{\circ} \mathrm{C}$ step. As a result, the procedure required new polymerase between each cycle. This limitation was overcome a few years later when Saiki et al. [20] utilized a thermostabile DNA polymerase [21] isolated from Thermus aquaticus [22]. With this refinement, scientists could conduct the DNA amplification reaction at high temperatures without adding new enzyme between each round of the PCR cycle. The higher amplification temperature also permitted more precise targeting of the DNA and reduced the incidence of primer dimers. Combined with the in vitro development of reverse transcription (RT) of mRNA into complementary DNA (cDNA) [23-26], detailed investigations of target transcripts became feasible. In order to visualize the PCR product(s), the samples were separated using gel-electrophoresis [27-29].

The sensitivity of PCR was clearly demonstrated by Li et al. [30] who, in 1988, analyzed genomic DNA of single sperm cells collected through a glass capillary. Two years later, Brady et al. [31] were able to analyze gene transcripts from single macrophages. This ability, to amplify and analyze transcripts from single cells, was taken one step further when Eberwine et al. [32,33] and Lambolez et al. [34] combined patch-clamp recordings with single-cell RT-PCR. Eberwine et al. utilized acutely dissociated neuronal cells obtained from the hippocampus of neonatal rats. The patch pipette served two purposes: to deliver oligo(dT) (with T7 recognition), deoxynucleoside triphosphates (dNTPs) and RT enzyme (Avian myeloblastosis virus), and to insulate the electrode solution needed to perform the electrophysiological recordings. Following the electrophysiological recordings, negative pressure was applied through the patch pipette and the cytosol was carefully collected with the pipette for nucleic acid amplification. In these experiments, several rounds of pre-amplification using T7 RNA polymerase in isothermal conditions were performed to increase the transcript concentration prior to the PCR. This approach allowed Eberwine et al. [33] to qualitatively detect transcripts of specific $\mathrm{Ca}^{2+}$ channels, $\gamma$-aminobutyric acid (GABA) receptors, $\mathrm{K}^{+}$channels, and $\mathrm{Na}^{+}$channels, as well as G-protein subunits and transcription factors c-jun and c-fos. The same group also conducted semi-quantitative measurements of transcript levels by measuring the relative intensity of the ethidium bromide (EtBr)-stained PCR products at the end of the PCR. However, as will be explained in the following sections, this method is unreliable and the quantitative results should be interpreted with caution. Lambolez et al. [34] used a slightly different approach to characterize several forms of AMPA receptors and their splice variants. Instead of pre-amplification of RNA, two rounds of PCR were conducted. Following the first round of PCR to amplify large fragments of the cDNA template, internal or nested primers were used to amplify a smaller fragment from the first PCR product. Similar to pre-amplification using T7 RNA polymerase, the PCR pre-amplification strategy also increases the amount of product needed to detect low abundance transcripts. 
Although the technical difficulty of investigating low-level transcripts was now resolved, the challenge of quantitatively measuring transcript levels remained. Traditionally, gene quantification was performed at the so-called plateau phase of the PCR at the end of a PCR assay (semi-quantification). However, as discovered by Higuchi and co-workers $[35,36]$ this plateau phase differs among replicated samples and was first discovered when Higuchi and co-workers started experimenting with the possibility of monitoring the PCR continuously, or in real-time during each amplification cycle [35,36]. By adding $\mathrm{EtBr}$ to the PCR reaction and using a charge-coupled device (CCD) camera, every PCR cycle could be monitored as a function of increasing fluorescence. It was clearly shown that after the initial exponential phase, the PCR enters a linear phase followed by a plateau phase [36-38]. This plateau phase results from inhibition of the PCR [37,38] and sample-to-sample variation, causing imprecise quantitative calculations [39]. When monitoring the PCR in real-time (i.e., at each cycle), however, it became possible to calculate the starting amount of the DNA template based on the exponential phase of the PCR curve. Additionally, using the exponential phase, rather than the plateau phase, increased the dynamic range. Currently, real-time PCR analysis is usually based on the PCR crossing point (quantification cycle), Cq. Cq is defined as the PCR cycle-number at which the signal monitoring the process reaches a predefined threshold level. Older terms that have also been used as the basis for calculating the amount of DNA/cDNA starting material include threshold cycle, $C_{\mathrm{t}}$, and crossing point, $C \mathrm{p}$ (see [40-42]).

The sensitivity of the quantitative (q) PCR assay is dependent on the specific labeling of the DNA/cDNA. Previously, EtBr was the preferred dye because of its strong shift in fluorescent intensity when bound to DNA. However, the use of novel dyes were already beginning to make their way by the mid-1990s. One of these dyes was SYBR green I, which greatly improved sensitivity [43-45]. Whereas $7000 \mathrm{ng}$ of 40-basepair DNA is needed to give a visible signal on a gel using EtBr, less than $14 \mathrm{ng}$ is needed when using SYBR Green I. In addition, Karlsen et al. [43] showed that SYBR green I was less dependent on the length of the DNA, thus generating similar fluorescence levels among short and long DNA fragments. In addition to novel DNA-specific dyes, several target-specific labeling strategies have been developed for qPCR (explained in the following sections), including gene specific probes [46]. Extensive work has also been conducted to standardize qPCR procedures, including laboratory practices and data analysis (See description of the MIQE-guidelines [42]. Such advances have led to qPCR becoming the gold standard for quantifying gene expression levels, both in research and in diagnostics.

In the present review, our main focus will be on the different strategies used for obtaining single cells or cell content from tissue slices or from dispersed cell cultures as a basis for gene expression analyses. We will then discuss strategies for optimizing RT and qPCR based on material from single cells.

While this review focuses on single-cell qPCR, several of the discussed methods are highly relevant for researchers exploring single-cell RNA-sequencing. However, we will not discuss RNA-sequencing per se but encourage the readers to study recent research papers and reviews specifically on this topic [47-55]. 


\section{Single-Cell Isolation and Harvesting Strategies}

Harvesting and securing the small amount of RNA molecules found within a single cell requires meticulous laboratory practice. In our laboratory we utilize separate rooms for RNA and cDNA/DNA handling. All equipment and experimental hardware are treated with RNase-inactivating reagents, like RNaseZAP (Ambion, TX, USA). In addition, we only use certified RNase-free aerosol-resistant filter tips, tubes, and reagents. All glassware is baked overnight at $220{ }^{\circ} \mathrm{C}$, including glass capillaries used for making cell harvesting pipettes and patch electrodes. Over time, DNA contaminations may also lead to false positives. Therefore, decontamination strategies should include DNA degrading detergents.

As mentioned above, two of the initial strategies to obtain DNA or RNA from single cells used glass capillaries. These methods involve either harvesting the whole cell, or via patch clamping, harvesting only the cell's content or cytosol [30,33]. Additional methods include isolating cells using laser-assisted micro-dissection [56-59], or by utilizing fluidics techniques, such as fluorescent-activated cell sorting (FACS) [60,61] and microfluidic technology utilizing polydimethylsiloxane (PDMS)-based lab-on-a-chip plates [62-66]. An overview of the different technologies are given in Table 1.

\section{Fluidics Technology}

The user-friendly environment and high throughput of fluidics technology compared to cell and cytosol harvesting with using glass capillaries have made these methods favorable in many applications.

Table 1. Overview of different cell and cytosol harvesting techniques.

\begin{tabular}{|c|c|c|c|c|}
\hline Method & $\begin{array}{l}\text { Equipme } \\
\text { nt Costs }\end{array}$ & $\begin{array}{l}\text { Laboratory } \\
\text { Skills }\end{array}$ & $\begin{array}{c}\text { Throuhgp } \\
\text { ut }\end{array}$ & Tissue \\
\hline FACA & High & Normal & High & Dissociated cells (in vitro) \\
\hline Microfluidics & High & Normal & High & Dissociated cells (in vitro) \\
\hline $\begin{array}{c}\text { Laser assisted } \\
\text { microdissection }\end{array}$ & High & High & Low & $\begin{array}{l}\text { Intact fixed and live tissue } \\
\text { (in vitro/ex vivo) }\end{array}$ \\
\hline Whole cell harvesting & Low & Normal & Medium & Dissociated cells (in vitro) \\
\hline $\begin{array}{l}\text { Harvesting of cytosol } \\
\text { using patch pipette }\end{array}$ & High & High & Low & $\begin{array}{l}\text { Intact live tissue } \\
\text { (in vitro/ex vivo) }\end{array}$ \\
\hline
\end{tabular}

FACS sorting of cells allows separation or sorting of heterogeneous cells into different containers or distribution of individual cells onto multi-well plates (Figure 1). Before separation, the cells are labeled with different fluorescent probes depending on the cytometry equipment and on the experimental setup. For instance, if the setup has three lasers, up to twelve different parameters can be quantitatively assaye, including viability, apoptosis, necrosis, intracellular $\mathrm{Ca}^{2+}$ signaling, membrane potential, and cell cycle stage (see review by Herzenberg et al. [61]). The most common lasers are the $488 \mathrm{~nm}(>20 \mathrm{~mW})$ and $633 \mathrm{~nm}(>18 \mathrm{~mW})$. However, depending on the experiments several additional lasers may be used including $375 \mathrm{~nm}(>7 \mathrm{~mW}), 405 \mathrm{~nm}(>50 \mathrm{~mW})$, 
and $561 \mathrm{~nm}(>18 \mathrm{~mW})$. The fast flowing liquid allows for single-cell separating before passing between one or several lasers and a detector. As individual cells pass, the detector measures light scatter from the emitting fluorophores. Depending on the selected characteristics, each droplet of liquid containing a single cell is given a charge, allowing cells to be separated into separate collecting tubes by an electric field just downstream of the laser-detector system. One disadvantage of this approach is that cells or cell cultures must be subjected to stimulation experiments and treated in a separate environment before FACS analysis.

To overcome the one experiment-one machine paradigm, a novel concept of a "total chemical analysis system" (TAS) utilizing microfluidics (often termed $\mu$ TAS) has emerged. The commercially available platform provided by Fluidigm is based on single-phase microfluidic systems using multilayer soft lithography (Figure 2). Multilayer soft lithography allows for compartmentalization of the cells by making and controlling small channel valves [67]. Another promising technology to handle small volumes of fluids is droplet-based microfluidics [68,69]. Depending on the technology, $\mu \mathrm{TAS}$ has the potential to provide different microenvironments where cells are grown and stimulated in small chambers whereupon either programmable valves regulate solution flow in or out of the chamber, or using droplet-based technology, the cells are guided to successive chambers for downstream experiment and analysis. These techniques may soon allow automated patch-clamp recordings and intracellular $\mathrm{Ca}^{2+}$ measurements [62,70], followed by transcriptome analysis in one chamber and proteomics analysis in another [66,71,72]. Microfluidics has also been applied to cells grown in monolayers or in three-dimensional environments, opening novel possibilities to explore intercellular communication.

Despite the great potential in single-cell analysis using $\mu$ TAS microfluidics, the method is limited by the range of cells that can be used; in particular, the fixed chamber size found on the micro plates can limit the use of variable cell sizes. In addition, because the $\mu$ TAS technology is still in its infancy, commercial systems offering multi-experimental microfluidic chips are limited to proof-of-concept. A few companies like Fluidigm have made several automated instruments for single-cell gene expression analysis, including sequencing. Their platforms are constructed of devices able to isolate single cells followed by lysis chips that can analyze gene sequence and expression from single cells. 


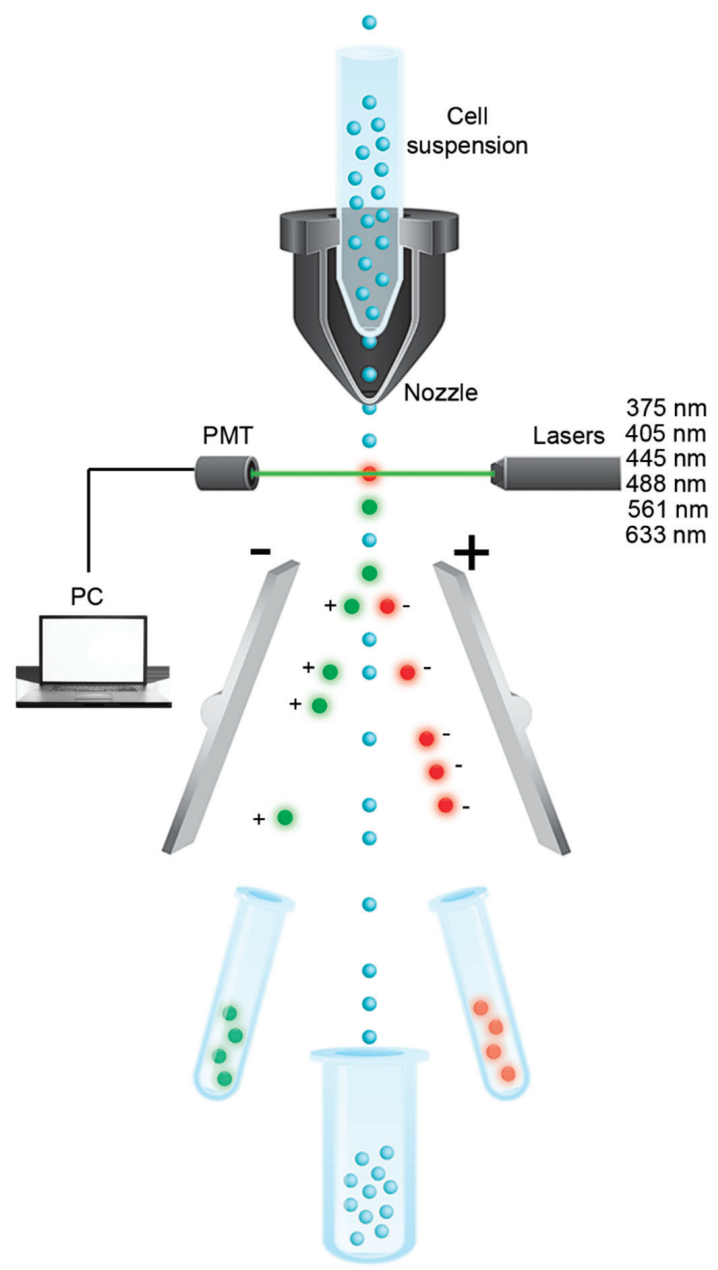

Figure 1. Fluorescence-activated cell sorting (FACS). The suspended cells are subjected to different fluorescent tags depending on the experimental set-up. As the cells flow in a stream of liquid they pass through a laser-detector system that monitors the fluorescent and light scatter characteristics. Based on their characteristics cells are separated in an electric field and into different collecting tubes or multiwell plates. Depending on the experiment a variety of markers may be used for separating the cells of interest. Demonstrated in this figure are cells tagged with green and red fluorescent proteins. Cells that are not labeled are separated into a third column/tube. 


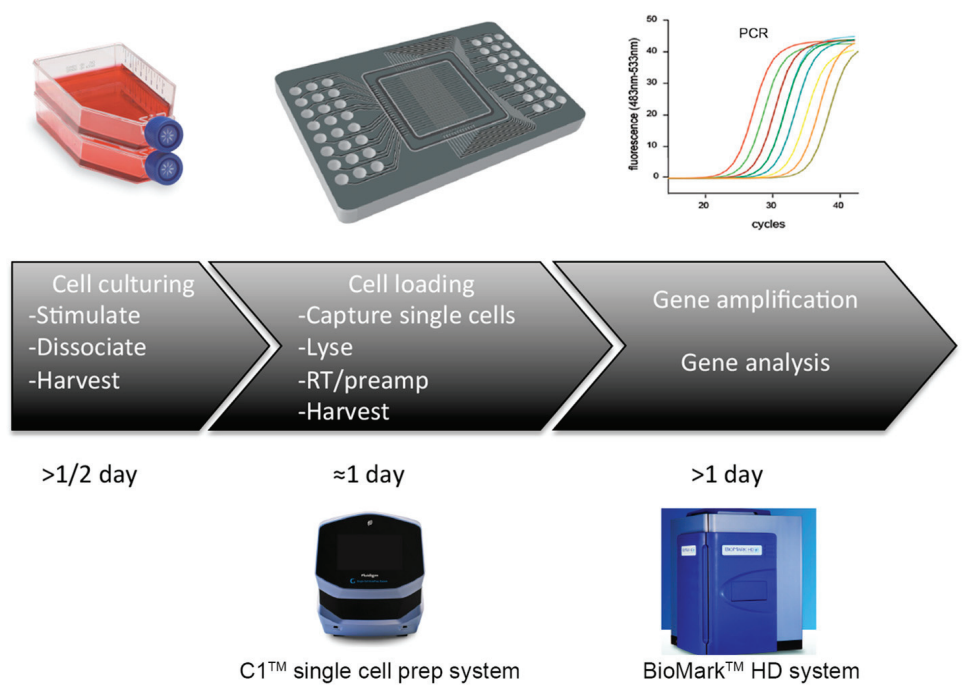

Figure 2. Microfluidics. Lab-on-a-chip technology allows for high-throughput screening in a microenvironment using small volume chambers to conduct different experiments. (Upper) similar to FACS, the cells need to be dissociated prior to the experiments; (Middle) the cells are typically placed in a chamber and, depending on the technology, the cells may be separated into different chambers containing only one cell; (Lower) the technology currently used by Fluidigm are single-phase microfluidic systems using multilayer soft lithography to make on and off valves to compartmentalize the cells. Another promising technology is the droplet-based microfluidics to handle small volumes of fluids. (Right) following single-cell isolation, lysis and cDNA synthesis the samples are subjected for gene analysis. A typical qPCR profile is shown with different colors representing the amplification curve of target gene(s).

\section{Single-Cell Laser-Assisted Microdissection}

While microfluidics are dependent on dissociated cells or cells removed from their natural environment, laser-assisted microdissection methods and cytosol harvesting through a patch-clamp pipette (see below) can be used in intact tissue [56]. In addition, laser-assisted microdissection does not require enzymatic dissociation of cells, making it less prone to disrupting intracellular signaling pathways. The cells are harvested under direct microscopic visualization and the method can be used on both live and fixed tissues (Figure 3) [57-59,73]. Today, there are four slightly different technologies that allow for precise laser dissection: laser microbeam microdissection (LMM), laser pressure catapulting (LPC), microdissection of membrane-mounted tissue (MOMeNT), and laser capture microdissection (LCM) (reviewed by [73]). All four technologies use a controllable pulsating laser coupled to a microscope allowing precise dissection of target cell(s). Depending on the equipment, the laser can be controlled by moving the objectives, by moving the microscope stage, or by using a dichroic mirror. The laser creates a cutting width of around $1 \mu \mathrm{m}$. One of the main challenges in single-cell laser assisted microdissection analysis is, therefore, the ability to 
dissect only the cell of interest and avoid contamination with neighboring cells or other unspecific fragments. This challenge can result in both false positives and false negatives. Dissecting too conservatively may lead to a cut not encompassing the entire cell, resulting in insufficient RNA harvest for downstream analysis. Dissecting too liberally may, on the other hand, lead to inclusion of unwanted RNA into the sample.

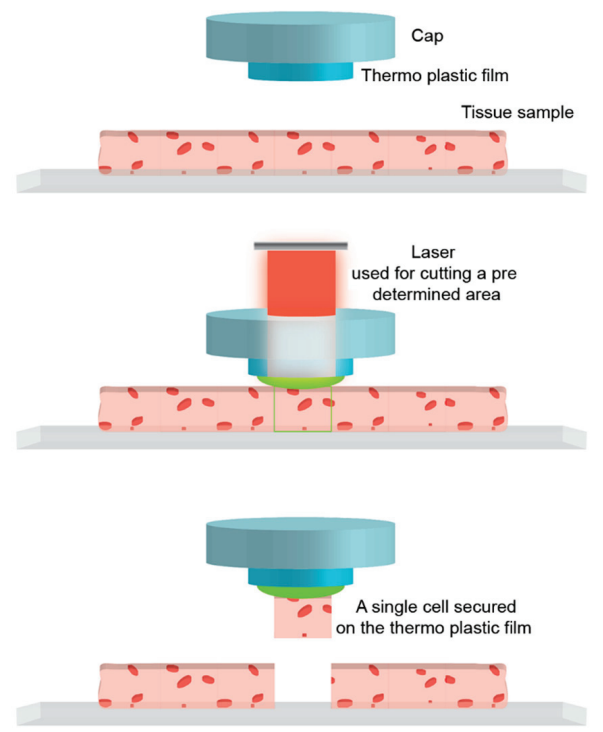

Figure 3. Single-cell laser-assisted microdissection. (Upper) The technique utilizes a laser placed on a microscope for visual identification; (Middle and lower) the laser can be controlled to dissect out target cells in fixed and live tissue. Several different technologies exist on how the laser works and how the target cell is collected following dissection.

\section{Harvesting Cells or Cytosol through Glass Capillary}

Similarly to laser assisted microdissection, harvesting of whole cells or cell cytosol using a glass capillary allows direct visualization of the target cell through a microscope. The harvesting of whole cells assures complete cell isolation and minimizes loss of already-limited amounts of RNA before the RT step (Figure 4) [6,74-76]. The method typically uses a controllable piston system coupled to a micromanipulator $[6,75]$. To avoid delays when adjusting the harvesting pressure the piston system should contain a non-compressible liquid, such as mineral oil. The glass capillary used for the harvesting can be made using a horizontal or vertical puller and the final diameter should be about $1 / 3$ of the cell diameter. The glass can also be polished using heat to avoid sharp edges. In addition, the tip can be modeled to a specific angle to improve harvesting and reduce the possibility of collecting surrounding solution. However, even with an optimized harvesting pipette, precautions should be taken. When collecting whole cells a small amount of the surrounding extracellular solution will follow into the collecting pipette [75]. This collected solution may 
contain contaminants like unwanted RNA. In fact, during our own work of optimizing single-cell qPCR assays we compared harvesting strategies on primary dispersed cells from pituitary with an immortalized pituitary cell line. We discovered that unwanted RNA contamination was dependent on the type of cell culture used. When harvesting whole cells from dissociated primary cell cultures there was a substantial amount of RNA present in the cellular bath. This contamination introduced false positive results in almost all of our samples. Even performing control experiments by solely resting the pipette in the bath for a few minutes had the potential of transferring unwanted RNA to our samples. However, when using the rat pituitary tumor GH4 cell line, we were able to collect whole cells without introducing false positives. We believe that these contradicting observations are a result of the relatively rough mechanical handling following chemical (trypsin, collagenase, etc.) treatment needed to dissociate tissues into single cells, as compared to the gentle pipetting sufficient for detaching cells like GH4 from the dish surface. In addition, most cell lines are usually well attached to plastic and glass surfaces making it easier to properly flush or wash the culture plates with clean incubation solution prior to collecting the cell of interest. Still, several groups have used whole cell harvesting on primary cell cultures seemingly without RNA contamination [6].

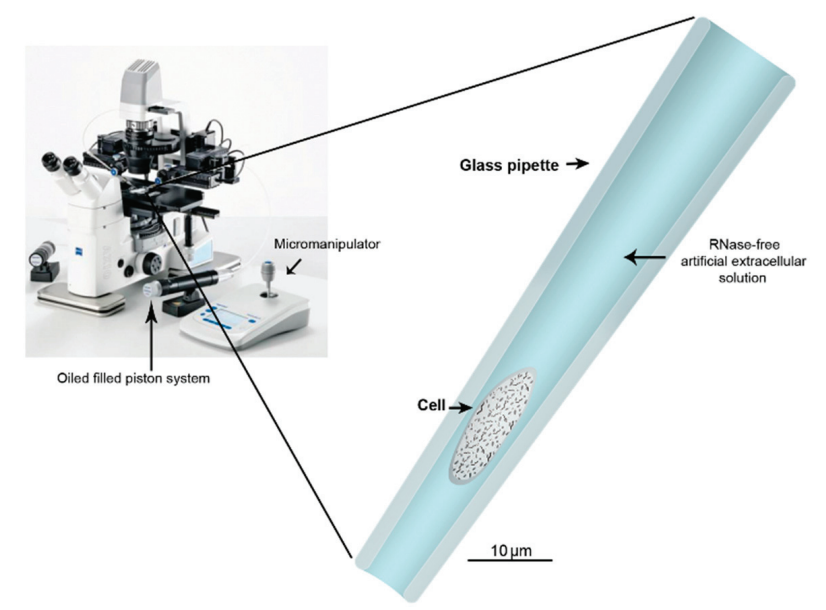

Figure 4. Harvesting of single cells in culture. The technique provides an economical and simple to use platform for harvesting single dissociated cells in culture. (Left) the cells are monitored under a microscope; (Right) using a glass pipette connected to a micromanipulator single cells can be collected relatively easily and transferred to a new tube for lysis and cDNA synthesis. The photograph of a typical microscope set-up is modified from Eppendorf.

To avoid aspiration of cell incubation medium or extracellular fluids, the cytosol can be harvested using the patch-clamp technique (Figure 5). Similar to harvesting of the whole cell, patch-clamping utilizes a glass pipette that is heat-pulled from a capillary to narrow the tip diameter. However, the tip is narrower than that in pipettes used for collecting whole cells. This 
narrow tip is positioned at the cell membrane. During optimal conditions, a tight interaction in the gigaohm range between the cell and the tip of the glass allows even small currents across the membrane to be recorded while also creating a barrier between the fluids surrounding the cell and the cell cytosol. To access the cytosol, a sub-atmospheric pressure can be created through the pipette, rupturing the membrane inside the patch. As mentioned, we experienced that RNA can attach to the glass surface and introduce false positives in the subsequent PCR analyses. To avoid this problem we silanize the patch pipette glass using Sigmacote [75]. However, combining patch-clamp experiments and subsequent single-cell RNA harvesting faces another problem. As a result of the large pipette volume, cytosolic factors are quickly diluted when using whole cell configurations. Since these cytosolic factors are important regulators of ion channel activity, researchers are often turning to the so-called perforated patch configuration where a perforating agent is added to the patch pipette. In this situation, the perforating agent makes small pores in the membrane within the patched membrane. This method leaves the cell interior preserved but does not provide access to the RNA. To overcome this obstacle and combine perforated patch-clamp recordings with subsequent cellular RNA collection, we found that substituting Amphotericin B with the saponin $\beta$-escin as the perforating agent, we could preserve the high resistance gigaohm seal when going from perforated patch to whole cell configuration. This transition was conducted in a similar way as when creating a normal whole cell configuration following formation of the gigaohm seal, simply by using gentle suction through the pipette. Thus, by combining whole cell configuration using silanized patch pipettes we were able to harvest the cell cytosol without the risk of collecting extracellular contaminants (Figure 6). Even though most of the RNA will be contained in the pipette using this approach, sample-to-sample variation can occur as a result of the harvesting. Therefore, quantitative measurements should be used with caution.

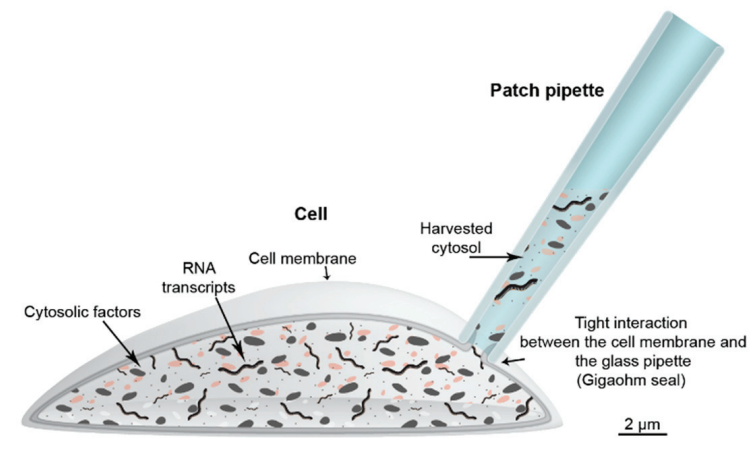

Figure 5. Harvesting of the cell's cytosol using a patch pipette. The technique is usually performed on tissue slices and combines patch-clamp electrophysiological recordings with analysis of gene transcripts. Following the electrophysiological experiments the cytosol from the cell may be harvest into the pipette using gentle suction. When the harvesting is finalized the tip of the pipette is withdrawn from the cell. During the final process membrane fragments reseals the tip of the patch pipette and protects the harvested RNA from contaminations in the surrounding solution. Silanizing the glass surface will also prevent extracellular RNA from attaching to the pipette. 


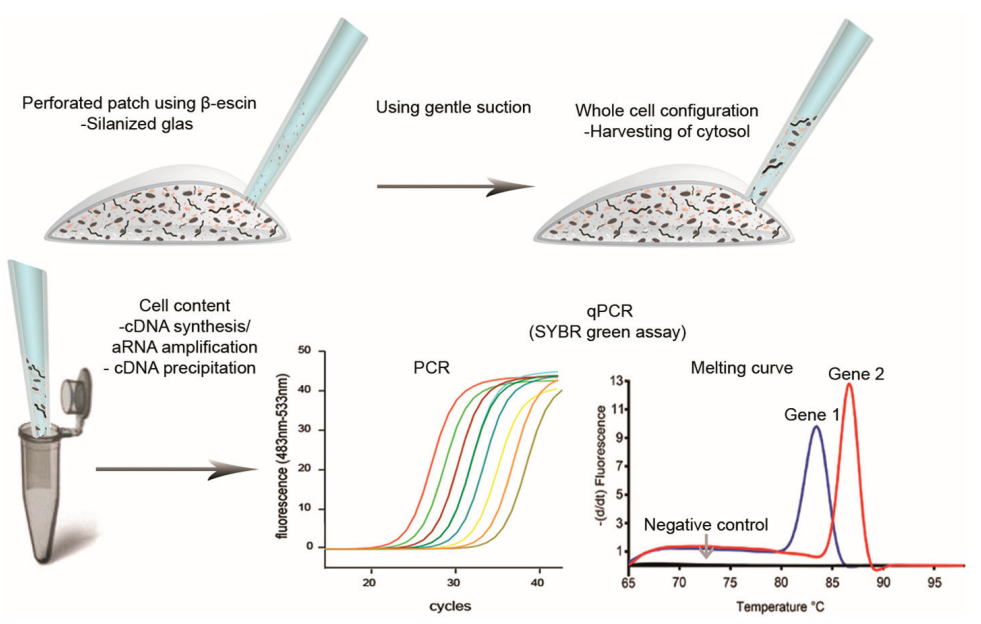

Figure 6. Schematic overview of the protocol used in our laboratory when performing perforated patch-clamp experiments followed by single-cell qPCR. This procedure allows for using perforated patch-clamp recordings and minimizes the incidents of false positive results during gene analysis. The patch pipette is silanized by briefly exposing the tip of the glass in a 1/10 dilution of Sigmacote. To allow for a tight high-resistance interaction between the glass and cell membrane, called a gigaohm seal, the glass tip needs to be fire polished using a microforge. (Upper left) The pipette is filled with an RNase-free solution suitable for the experiments and using $\beta$-escin to perforate the cell membrane; (Upper right) the cell cytosol can be harvested following transition to whole cell configuration using gentle suction; (Lower left) the cell content is transferred to a $0.5 \mathrm{~mL}$ tube containing RNA stabilizing solution; (Lower middle figure) the target genes are amplified using qPCR (colored curve represents target gene been amplified); (Lower right) following qPCR amplification a melting curve analysis is performed by gently heating the PCR product(s) from 65 to $98^{\circ} \mathrm{C}$ while continually reading of the fluorescent (The curve(s) are plotted as the negative 1. derivative of fluorescent with respect to temperature).

\section{Lysis and Securing the RNA}

Unless the cytosol is harvested using a patch pipette, cells need to be lysed in order to access the RNA for RT. Cell lysis must be efficient, yet not interfere with downstream processes. Today, several methods have been used for lysing single cells, The methods including optical, acoustic, electrical, mechanical, and chemical lysis (for review see [77]). The benefit of non-chemical lysis is that the methods are buffer independent. This means that the buffer can be optimized for the downstream processes such as RT. However, with the exception of chemical lysis, most of these methods are developed and validated for use with microfluidics technologies, including capillary electrophoresis. Cells collected using a glass pipette, FACS, or laser-assisted cell harvesting are typically chemically lysed, (e.g., $[6,75,78,79]$ ) where a detergent generates small pores in the 
membrane. Since the different detergents differ both in general structure (e.g., ionic, non-ionic and zwitterionic moieties) and their ability to interact and lyse the cell, it is important to validate and test the detergent in use. Several detergents, such as the anionic sodium dodecyl sulphate (SDS), or the cationic ethyl trimethyl ammonium bromide, lyse cells quickly, often within seconds, but also have the tendency to denature proteins potentially disturbing the RT enzyme. Other detergents are non-denaturing, including 3-[(3-cholamidopropyl)dimethylammonio]-1-propanesulfonate (CHAPS/CHAPSO), Triton, and Nonidet P-40/IGEPAL CA-630. In addition, several manufacturers deliver a variety of ready-to-use lysis buffers optimized for small number of cells, down to single-cells, in combination with RT. Several cell lysis strategies have also used high concentrations $(>4 \mathrm{M}$ ) of guanidine salts because of their ability to inactivate nucleases and free the nucleic acids from bound proteins [80-82]. One disadvantage of this approach is that the RNA then needs to be purified, as a result of the detrimental effect guanidine salts have on proteins including the RT enzyme. However, Bengtsson et al. [83] demonstrated that low volumes and concentrations (1-2 $\mu \mathrm{L}$ and $0.5-1 \mathrm{M})$ of guanidine thiocyanate (GuSCN) efficiently lysed single pancreatic cells. Prior to the RT step, the GuSCN was diluted down to about $40 \mathrm{mM}$, thereby avoiding the need for RNA cleanup. At this concentration GuSCN even improved the conditions for RT, and the authors concluded that GuSCN serves both as a cell lysis agent and RNase inhibitor. Recently, Svec et al. [78] performed a comprehensive study by comparing several detergents, lysis solutions and column-based RNA isolation. The experiments were conducted using single FACS-sorted astrocytes collected into 96-well plates with $5 \mu \mathrm{L}$ lysis buffer per well. The evaluated solutions were 7-deaza-2'-deoxyguanosine-5'-triphosphate lithium salt $(100 \mu \mathrm{M})$, Betaine solution $(4 \mathrm{M})$, bovine serum albumin (BSA) (1-4 mg/mL), guanidine thiocyanate solution (40-80 mM), GenElute linear polyacrylamide (50 ng/ $\mu \mathrm{L})$, Igepal CA-630 (0.5\%-4\%), and polyinosinic acid potassium salt $(50 \mathrm{ng} / \mu \mathrm{L})$. Interestingly, BSA was sufficient for single-cell lysis and compatible with both RT and qPCR. Earlier studies have also demonstrated that BSA efficiently buffers inhibitory factors and can improve PCR efficiency [84-89]. Since GuSCN and BSA have several positive effects downstream of cell lysis they could potentially improve conditions for single-cell analysis where only the cytosol is harvested and transferred for RT. In our experience, the cell content harvested following patch-clamp recordings may be expelled directly into a storage solution containing the relatively weak chelating agent citrate and a thermostabile RNase inhibitor. The low $\mathrm{pH}$ and chelating properties of citrate reduces RNA base hydrolysis. In addition, because we use random hexamers to prime the RNA for cDNA synthesis, the RNA need to be heated for several minutes at $65^{\circ} \mathrm{C}$. By using a relatively heat-stable RNase inhibitor we can add the inhibitor at an earlier step than is recommended in the protocol developed by ThermoFisher Scientific/Invitrogen. Importantly, EGTA and EDTA should be avoided because their strong chelating properties reduce free $\mathrm{Mg}^{2+}$ levels to below the requirements of downstream enzymes, like reverse transcriptase.

\section{Reverse Transcription}

Three basic strategies are used when priming RNA for RT are oligo(dT), random hexamer primers (or a combination of these), and gene specific primers. Earlier reports have suggested that random hexamers may be less efficient compared to oligo(dT) nucleotides that are specific for the 
polyA tail of mRNAs [90]. However, priming with oligo(dT) will only generate cDNA from RNA containing a polyA tail. If the starting material contains small numbers of transcripts from individual cells, and heat treatment is used for cell lysis, then the prevalence of fragmented mRNA may decrease. Random hexamers, on the other hand, will bind to all complementary regions of an RNA fragment increasing the likelihood of converting all RNA fragments into cDNA, including those targeted by gene specific primers in the subsequent PCR. A combination of the different primers may also be used and could be beneficial when performing gene analysis on single cells [91].

The amount of mRNA from single cells is limited to between $10^{5}-10^{6}$ molecules [92] and the isolation is often time consuming, rendering the RNA from each cell valuable. Therefore, to avoid multiple sampling in order to analyze several genes from a single cell type, pre-amplification is often necessary. As mentioned above, two strategies are commonly used for increasing the number of transcripts. In the strategy developed by Vangelder et al. [32], the authors used oligo(dT) primers comprising a promoter recognized by the bacteriophage DNA-dependent RNA polymerase T7. Following cDNA synthesis RNase $\mathrm{H}$ hydrolyzes the template RNA leaving single stranded cDNA. Under isothermal conditions, the T7 synthesizes a new RNA strand from the cDNA template. Since only the initial cDNA template contains the T7 promoter, the template concentration itself does not increase, making the amplification process essentially linear. New and improved promoters and reaction buffers for RNA amplification have reduced nonspecific activity and increased cDNA yield (see e.g., [93-97]). However, pre-amplification involves multiple steps and is, therefore, labor intensive. A less time consuming strategy is to use two rounds of PCR, as demonstrated by Lambolez et al. [34]. However, in their study they could only target a few genes defined by the primers [34]. Therefore, they developed an improvement of the method using homomeric tailing of the cDNA with polyA and subsequent PCR (global amplification) with oligo(dT) primers [31,98]. In fact, Iscove et al. [99] demonstrated that by using this strategy they could preserve abundance relationships through amplification as high as $3 \times 10^{11}$-fold. Further, compared to linear amplification strategies, the RNA needed for microarray analysis could be reduced by a million-fold and give reproducible results using the picogram range of total RNA obtainable from single cells. Several reports have tested and validated different amplification strategies [100,101] including the so-called switching mechanism of 5'end of RNA template PCR (SMART PCR) [102]. Both linear pre-amplification and SMART PCR have been used in singlecell RNA sequencing experiments [103-107].

\section{8. qPCR}

Several detection formats can be used in qPCR. These include fluorescent dyes, such as SYBR green, which bind to any double-stranded DNA, [90], and sequence-specific probes (see review by [108]). The advantages of using probes are that fluorescence is emitted only during specific binding and that several genes can be detected in the same reaction [109]. The main disadvantages are the cost and the fact that a melting curve analysis (explained below) cannot be performed directly following PCR. SYBR green or other non-specific DNA-binding fluorescent dyes, on the other hand, may be used with any gene-specific primer pair. Compared to probe-based qPCR, the 
widely-used SYBR green is more cost-efficient. SYBR green binds to the minor groove in double-stranded DNA and, once bound, the signal increases 1000 times compared to free dye in solution. As SYBR green binds to any double-stranded DNA, including primer dimers, the qPCR assay must be carefully validated. To discriminate different products, a melting curve analysis of the products is usually performed directly after the PCR without breaking the sealed samples, eliminating carry-over contamination or pipetting errors. The melting temperature of the PCR product is based not only on the product size, but also on the GC content and the distribution of GC within the PCR product. This is favorable compared to gel-electrophoresis, which can only separate the products based on size. The specificity of melting curve analysis reduces the risk of false positives and can be used to separate products with minor differences, such as point mutations.

Specificity, sensitivity, and efficiency of qPCR are dependent on numerous factors including priming strategies, purity of cDNA, as well as number and length of the PCR cycle(s). In our laboratory we utilize the freeware Primer3plus [110] to design gene-specific primers. In addition, we routinely perform in silico testing of all primers using software like Vector NTI [111] or similar Following initial screening, the primers are validated and the optimal primer annealing temperature is determined using cDNA synthesized from total RNA extracted from tissue. In general, lowering the annealing temperature increases sensitivity and efficiency. However, too low of a temperature can create nonspecific primer binding and give false positives. These parameters are measured using serial dilution curves of cDNA. The $\mathrm{Cq}$ can be plotted against the logarithm of the relative concentration of the cDNA starting material. The efficiency of the qPCR assay can then be described by the slope of the regression line (efficiency $=10^{-1} /$ slope). If the slope of the dilution curve is -3.32 , the efficiency equals 2 , meaning that each PCR cycle doubles the product. If the efficiency is 2 , or $100 \%$, a $10 \times$ dilution of cDNA starting material will give a change in $\mathrm{Cq}$ $(\Delta \mathrm{Cq})$ of 3.2 .

Due to the limited amount of transcript, single-cell qPCR is often conducted using undiluted cDNA as template. This can result in accumulated levels of DTT and RT enzyme, which inhibit and profoundly affect the qPCR assay performance [112-114]. To avoid these inhibitory factors, a protocol for single-cell cDNA precipitation was developed by Liss [113]. Introducing this cDNA precipitation step into our own single-cell analyses has greatly reduced the incidence of inconclusive qPCR results. Notably, adding a known concentration of non-expressed synthetic RNA-spike can be used for validating the workflow process downstream of cell lysis including the precipitation.

\section{Quantitative Gene Analysis}

The nature of gene regulation within a single cell prevents relative quantification normalized to so-called housekeeping genes. For reliable quantification Bengtsson et al. [83] developed a protocol for absolute quantification based on a known standard. The genes of interest are cloned and amplified by PCR before determining the concentration spectrophotometrically (A260). A series of dilutions is made before qPCR with the diluted DNA as template. The template needs to be pure and the copy number can be determined by using the average weight of a base $(660 \mathrm{~g} / \mathrm{mol})$. 


\section{Future Possibilities and Challenges}

Although much progress has been made during recent years in single-cell gene analysis, the field is still facing several challenges related to harvesting strategies, and to transcript amplification and analysis. Common to most stages and technologies is the need for improved reagents and more precise enzymes, e.g., reagents that avoid or reduce the potential for biased or non-linear pre-amplification of the transcripts. In addition, more powerful software focusing on genetic analysis of single-cell transcript variability needs to be developed.

Despite the challenges, the field of single-cell gene analysis is moving forward rapidly with continuous development of new hardware, software and reagents. In particular, we have seen a dramatic development in the field of nucleic acid sequencing. This development has resulted in more than 10 -fold reduction in costs for sequencing during the last decade. In parallel with this development integrated systems, like lab-on-a-chip technology, has facilitated single-cell analysis. Within the next decade multifunctional equipment, based on microfluidics technology, will probably reduce hands on time for sample preparation and create a more streamlined processing. The working platforms will likely perform several subsequent steps including cell stimulation and manipulation, automated patch-clamp electrophysiology, imaging, including $\mathrm{Ca}^{2+}$ measurements, nucleic acid amplification and sequencing, and possibly proteomics, again, reducing hands-on time related to manual transfer of samples between equipment. [62,70].

Despite the promising and broad applications of fluidics technology, it will most probably be limited to dissociated cells in suspension. Investigations on whole organ function and plasticity require that spatial integrity of the tissue is preserved, allowing investigations of temporal events. Intact, model organs generally rule out fluidics systems. However, as opposed to the rapid development of microfluidics, the development of equipment that allows single-cell harvesting from intact tissue has relied on older techniques, like harvesting of cytosol through a patch pipette. This slow progress may now come to a close with nanotechnology. Currently, the preferred method for isolating transcripts from live, intact tissues is harvesting of the cell cytosol using a glass patch pipette. Even though this has proved valuable, it is limited by the fact that the harvesting requires a continuous tight interaction between the tip of the glass and the cell membrane, which is often lost during harvesting (Figure 5). However, recent developments demonstrate the potential use of multiwalled carbon nanotubes mounted at the tip of conventional micropipettes [115]. Because these carbon nanotubes only have a fraction of the diameter used when making conventional patch-clamp pipettes, the nanotubes can, in a less invasive fashion, access the cytosol by penetrating the cell membrane without destroying the cell. However, the technology is still at the stage of "proof of principle", but has successfully been demonstrated to work as a cell-specific delivery system and used for electrophysiological experiments, e.g., [115,116]. With further development these tools may soon be commercially available.

\section{Summary}

Single-cell gene analysis is a highly-powerful approach to understand the dynamics of gene regulation. Depending on the research focus, several methods are available for harvesting or 
isolating single-cell RNA. The methods need to be carefully evaluated and considerations, like spatial and temporal gene regulation, can be affected by the chosen harvesting strategy. In addition, the sensitivity of PCR makes it prone for false positives, affecting the assay both qualitatively and quantitatively. In our experiments, in which the assay was designed for phenotyping cells based on their gene expression, we identified extracellular contamination that greatly affected the qPCR assay. Thus, thorough validation of the cell isolation process is as crucial as the validation of downstream processes.

Acknowledgments: This work was funded by the Research Council of Norway project number 244461 and by the Norwegian University of Life Sciences (NMBU). We would like to acknowledge Anthony Peltier for help with the illustrations and Dianne Baker for critically reading the manuscript.

Author Contributions: Finn-Arne Weltzien and Kjetil Hodne outlined the structure of the paper, Kjetil Hodne drafted the manuscript, Finn-Arne Weltzien and Kjetil Hodne commented and produced the final version.

Conflicts of Interest: The authors declare no conflict of interest.

\section{References}

1. Ko, M.S.H.; Nakauchi, H.; Takahashi, N. The dose dependence of glucocorticoid-inducible gene-expression results from changes in the number of transcriptionally active templates. EMBO J. 1990, 9, 2835-2842.

2. Walters, M.C.; Fiering, S.; Eidemiller, J.; Magis, W.; Groudine, M.; Martin, D.I.K. Enhancers increase the probability but not the level of gene-expression. Proc. Natl. Acad. Sci. USA 1995, 92, 7125-7129.

3. McAdams, H.H.; Arkin, A. Stochastic mechanisms in gene expression. Proc. Natl. Acad. Sci. USA 1997, 94, 814-819.

4. Elowitz, M.B.; Levine, A.J.; Siggia, E.D.; Swain, P.S. Stochastic gene expression in a single cell. Science 2002, 297, 1183-1186.

5. Norris, A.J.; Stirland, J.A.; McFerran, D.W.; Seymour, Z.C.; Spiller, D.G.; Loudon, A.S.I.; White, M.R.H.; Davis, J.R.E. Dynamic patterns of growth hormone gene transcription in individual living pituitary cells. Mol. Endocrinol. 2003, 17, 193-202.

6. Bengtsson, M.; Stahlberg, A.; Rorsman, P.; Kubista, M. Gene expression profiling in single cells from the pancreatic islets of Langerhans reveals lognormal distribution of mRNA levels. Genome Res. 2005, 15, 1388-1392.

7. Chubb, J.R.; Trcek, T.; Shenoy, S.M.; Singer, R.H., Transcriptional pulsing of a developmental gene. Curr. Biol. 2006, 16, 1018-1025.

8. Raj, A.; Peskin, C.S.; Tranchina, D.; Vargas, D.Y.; Tyagi, S. Stochastic mRNA synthesis in mammalian cells. PLoS Biol. 2006, 4, 1707-1719.

9. Raj, A.; van Oudenaarden, A. Nature, nurture, or chance: Stochastic gene expression and its consequences. Cell 2008, 135, 216-226. 
10. Method of the Year 2013. Methods to sequence the DNA and RNA of single cells are poised to transform many areas of biology and medicine. Nat. Methods 2014, 11, 1.

11. Malnic, B.; Hirono, J.; Sato, T.; Buck, L.B. Combinatorial receptor codes for odors. Cell 1999, 96, 713-723.

12. Raghunathan, A.; Ferguson, H.R.; Bornarth, C.J.; Song, W.M.; Driscoll, M.; Lasken, R.S. Genomic DNA amplification from a single bacterium. Appl. Environ. Microbiol. 2005, 71, 3342-3347.

13. Zhang, K.; Martiny, A.C.; Reppas, N.B.; Barry, K.W.; Malek, J.; Chisholm, S.W.; Church, G.M. Sequencing genomes from single cells by polymerase cloning. Nat. Biotechnol. 2006, 24, 680-686.

14. Navin, N.; Kendall, J.; Troge, J.; Andrews, P.; Rodgers, L.; McIndoo, J.; Cook, K.; Stepansky, A.; Levy, D.; Esposito, D.; et al. Tumour evolution inferred by single-cell sequencing. Nature 2011, 472, 90-94.

15. Zong, C.H.; Lu, S.J.; Chapman, A.R.; Xie, X.S. Genome-wide detection of single-nucleotide and copy-number variations of a single human cell. Science 2012, 338, 1622-1626.

16. Kleppe, K.; Ohtsuka, E.; Kleppe, R.; Molineux, I.; Khorana, H.G. Studies on polynucleotides. XCVI. Repair replications of short synthetic DNA's as catalyzed by DNA polymerases. J. Mol. Biol. 1971, 56, 341-361.

17. Rabinow, P. Making PCR: A Story of Biotechnology; University of Chicago Press: Chicago, IL, USA, 1996.

18. Saiki, R.K.; Scharf, S.; Faloona, F.; Mullis, K.B.; Horn, G.T.; Erlich, H.A.; Arnheim, N. Enzymatic amplification of $\beta$-globin genomic sequences and restriction site analysis for diagnosis of sickle-cell anemia. Science 1985, 230, 1350-1354.

19. Mullis, K.; Faloona, F.; Scharf, S.; Saiki, R.; Horn, G.; Erlich, H. Specific enzymatic amplification of DNA in vitro-The polymerase chain-reaction. Cold Spring Harb. Symp. Quant. Biol. 1986, 51, 263-273.

20. Saiki, R.K.; Gelfand, D.H.; Stoffel, S.; Scharf, S.J.; Higuchi, R.; Horn, G.T.; Mullis, K.B.; Erlich, H.A. Primer-directed enzymatic amplification of DNA with a thermostable DNA-polymerase. Science 1988, 239, 487-491.

21. Chien, A.; Edgar, D.B.; Trela, J.M. Deoxyribonucleic-acid polymerase from extreme thermophile Thermus aquaticus. J. Bacteriol. 1976, 127, 1550-1557.

22. Brock, T.D.; Freeze, H. Thermus aquaticus gen. n. and sp. n., a nonsporulating extreme thermophile. J. Bacteriol. 1969, 98, 289-297.

23. Baltimore, D. RNA-dependent DNA polymerase in virions of RNA tumour viruses. Nature 1970, 226, 1209-1211.

24. Temin, H.M.; Mizutani, S. Viral RNA-dependent DNA polymerase: RNA-dependent DNA polymerase in virions of rous sarcoma virus. Rev. Med. Virol. 1998, 8, 3-11.

25. Verma, I.M.; Temple, G.F.; Baltimor.D; Fan, H. In vitro synthesis of DNA complementary to rabbit reticulocyte 10S RNA. Nat. New Biol. 1972, 235, 163-167. 
26. Efstratiadis, A.; Kafatos, F.C.; Maxam, A.M.; Maniatis, T. Enzymatic in vitro synthesis of globin genes. Cell 1976, 7, 279-288.

27. Fisher, M.P.; Dingman, C.W. Role of molecular conformation in determining electrophoretic properties of polynucleotides in agarose-acrylamide composite gels. Biochemistry 1971, 10, 1895-1899.

28. Aaij, C.; Borst, P. The gel-electrophoresis of DNA. Biochim. Biophys. Acta 1972, 269, $192-$ 200.

29. Sharp, P.A.; Sugden, B.; Sambrook, J. Detection of two restriction endonuclease activities in haemophilus-parainfluenzae using analytical agarose-ethidium bromide electrophoresis. Biochemistry 1973, 12, 3055-3063.

30. Li, H.; Gyllensten, U.B.; Cui, X.; Saiki, R.K.; Erlich, H.A.; Arnheim, N. Amplification and analysis of DNA sequences in single human sperm and diploid cells. Nature 1988, 335, 414-417.

31. Brady, G.; Barbara, M.; Iscove, N.N. Representative in vitro cDNA amplification from individual hemopoietic cells and colonies. Methods Mol. Cell. Biol. 1990, 2, 17-25.

32. Vangelder, R.N.; Vonzastrow, M.E.; Yool, A.; Dement, W.C.; Barchas, J.D.; Eberwine, J.H. Amplified RNA synthesized from limited quantities of heterogeneous cDNA. Proc. Natl. Acad. Sci. USA 1990, 87, 1663-1667.

33. Eberwine, J.; Yeh, H.; Miyashiro, K.; Cao, Y.X.; Nair, S.; Finnell, R.; Zettel, M.; Coleman, P. Analysis of gene-expression in single live neurons. Proc. Natl. Acad. Sci. USA 1992, 89, 3010-3014.

34. Lambolez, B.; Audinat, E.; Bochet, P.; Crepel, F.; Rossier, J., AMPA receptor subunits expressed by single Purkinje cells. Neuron 1992, 9, 247-258.

35. Higuchi, R.; Dollinger, G.; Walsh, P.S.; Griffith, R. Simultaneous amplification and detection of specific DNA sequences. Bio/Technology 1992, 10, 413-417.

36. Higuchi, R.; Fockler, C.; Dollinger, G.; Watson, R. Kinetic PCR analysis: Real-time monitoring of DNA amplification reactions. Bio/Technology 1993, 11, 1026-1030.

37. McPherson, M.J.; Hames, B.D.; Taylor, G.R. PCR 2: A Practical Approach; Oxford University Press: Oxford, UK, 1995.

38. Kainz, P. The PCR plateau phase-Towards an understanding of its limitations. Biochim. Biophys. Acta 2000, 1494, 23-27.

39. Schmittgen, T.D.; Zakrajsek, B.A.; Mills, A.G.; Gorn, V.; Singer, M.J.; Reed, M.W. Quantitative reverse transcription-polymerase chain reaction to study mRNA decay: Comparison of endpoint and real-time methods. Anal. Biochem. 2000, 285, 194-204.

40. Livak, K.J.; Schmittgen, T.D. Analysis of relative gene expression data using real-time quantitative PCR and the $2^{-\Delta \Delta} C_{\mathrm{t}}$ method. Methods 2001, 25, 402-408.

41. Bustin, S.A. Quantification of mRNA using real-time reverse transcription PCR (RT-PCR): Trends and problems. J. Mol. Endocrinol. 2002, 29, 17.

42. Bustin, S.A.; Benes, V.; Garson, J.A.; Hellemans, J.; Huggett, J.; Kubista, M.; Mueller, R.; Nolan, T.; Pfaffl, M.W.; Shipley, G.L.; et al. The MIQE guidelines: Minimum information for publication of quantitative real-time PCR experiments. Clin. Chem. 2009, 55, 611-622. 
43. Karlsen, F.; Steen, H.B.; Nesland, J.M. SYBR green I DNA staining increases the detection sensitivity of viruses by polymerase chain-reaction. J. Virol. Methods 1995, 55, 153-156.

44. Heid, C.A.; Stevens, J.; Livak, K.J.; Williams, P.M. Real time quantitative PCR. Genome Res. 1996, 6, 986-994.

45. Wittwer, C.T.; Ririe, K.M.; Andrew, R.V.; David, D.A.; Gundry, R.A.; Balis, U.J. The LightCycler: A microvolume multisample fluorimeter with rapid temperature control. Biotechniques 1997, 22, 176-181.

46. Holland, P.M.; Abramson, R.D.; Watson, R.; Gelfand, D.H. Detection of specific polymerase chain-reaction product by utilizing the $5^{\prime}-3^{\prime}$ exonuclease activity of Thermus aquaticus DNA-polymerase. Proc. Natl. Acad. Sci. USA 1991, 88, 7276-7280.

47. Liu, P.; Mathies, R.A. Integrated microfluidic systems for high-performance genetic analysis. Trends Biotechnol. 2009, 27, 572-581.

48. Nagarajan, N.; Pop, M. Sequence assembly demystified. Nat. Rev. Genet. 2013, 14, 157-167.

49. Shapiro, E.; Biezuner, T.; Linnarsson, S. Single-cell sequencing-based technologies will revolutionize whole-organism science. Nat. Rev. Genet. 2013, 14, 618-630.

50. Van Loo, P.; Voet, T. Single cell analysis of cancer genomes. Curr. Opin. Genet. Dev. 2014, 24, 82-91.

51. Achim, K.; Pettit, J.-B.; Saraiva, L.R.; Gavriouchkina, D.; Larsson, T.; Arendt, D.; Marioni, J.C. High-throughput spatial mapping of single-cell RNA-seq data to tissue of origin. Nat. Biotechnol. 2015, 33, 503-509.

52. Buettner, F.; Natarajan, K.N.; Casale, F.P.; Proserpio, V.; Scialdone, A.; Theis, F.J.; Teichmann, S.A.; Marioni, J.C.; Stegie, O. Computational analysis of cell-to-cell heterogeneity in single-cell RNA-sequencing data reveals hidden subpopulations of cells. Nat. Biotechnol. 2015, 33, 155-160.

53. Crosetto, N.; Bienko, M.; van Oudenaarden, A. Spatially resolved transcriptomics and beyond. Nat. Rev. Genet. 2015, 16, 57-66.

54. Darmanis, S.; Sloan, S.A.; Zhang, Y.; Enge, M.; Caneda, C.; Shuer, L.M.; Gephart, M.G.H.; Barres, B.A.; Quake, S.R. A survey of human brain transcriptome diversity at the single cell level. Proc. Natl. Acad. Sci. USA 2015, 112, 7285-7290.

55. Stegle, O.; Teichmann, S.A.; Marioni, J.C. Computational and analytical challenges in single-cell transcriptomics. Nat. Rev. Genet. 2015, 16, 133-145.

56. EmmertBuck, M.R.; Bonner, R.F.; Smith, P.D.; Chuaqui, R.F.; Zhuang, Z.P.; Goldstein, S.R.; Weiss, R.A.; Liotta, L.A. Laser capture microdissection. Science 1996, 274, 998-1001.

57. Bonner, R.F.; EmmertBuck, M.; Cole, K.; Pohida, T.; Chuaqui, R.; Goldstein, S.; Liotta, L.A. Laser capture microdissection: Molecular analysis of tissue. Science 1997, 278, 1481-1483.

58. Schutze, K.; Lahr, G. Identification of expressed genes by laser-mediated manipulation of single cells. Nat. Biotechnol. 1998, 16, 737-742.

59. Podgorny, O.V. Live cell isolation by laser microdissection with gravity transfer. J. Biomed. Opt. 2013, 18, 8 .

60. Herzenberg, L.A.; Sweet, R.G. Fluorescence-activated cell sorting. Sci. Am. 1976, 234, $108-117$. 
61. Herzenberg, L.A.; Parks, D.; Sahaf, B.; Perez, O.; Roederer, M. The history and future of the fluorescence activated cell sorter and flow cytometry: A view from Stanford. Clin. Chem. 2002, 48, 1819-1827.

62. Wheeler, A.R.; Throndset, W.R.; Whelan, R.J.; Leach, A.M.; Zare, R.N.; Liao, Y.H.; Farrell, K.; Manger, I.D.; Daridon, A. Microfluidic device for single-cell analysis. Anal. Chem. 2003, $75,3581-3586$.

63. Ottesen, E.A.; Hong, J.W.; Quake, S.R.; Leadbetter, J.R. Microfluidic digital PCR enables multigene analysis of individual environmental bacteria. Science 2006, 314, 1464-1467.

64. Warren, L.; Bryder, D.; Weissman, I.L.; Quake, S.R. Transcription factor profiling in individual hematopoietic progenitors by digital RT-PCR. Proc. Natl. Acad. Sci. USA 2006, 103, 17807-17812.

65. Whitesides, G.M., The origins and the future of microfluidics. Nature 2006, 442, 368-373.

66. Wu, A.R.; Neff, N.F.; Kalisky, T.; Dalerba, P.; Treutlein, B.; Rothenberg, M.E.; Mburu, F.M.; Mantalas, G.L.; Sim, S.; Clarke, M.F.; Quake, S.R. Quantitative assessment of single-cell RNA-sequencing methods. Nat. Methods 2014, 11, 41-46.

67. Unger, M.A.; Chou, H.P.; Thorsen, T.; Scherer, A.; Quake, S.R. Monolithic microfabricated valves and pumps by multilayer soft lithography. Science 2000, 288, 113-116.

68. Joensson, H.N.; Svahn, H.A. Droplet microfluidics-A tool for single-cell analysis. Angew. Chem. Int. Ed. 2012, 51, 12176-12192.

69. Seemann, R.; Brinkmann, M.; Pfohl, T.; Herminghaus, S. Droplet based microfluidics. Rep. Prog. Phys. 2012, 75, 016601.

70. Pantoja, R.; Nagarah, J.M.; Starace, D.M.; Melosh, N.A.; Blunck, R.; Bezanilla, F.; Heath, J.R. Silicon chip-based patch-clamp electrodes integrated with PDMS microfluidics. Biosens. Bioelectron. 2004, 20, 509-517.

71. Hong, J.W.; Studer, V.; Hang, G.; Anderson, W.F.; Quake, S.R. A nanoliter-scale nucleic acid processor with parallel architecture. Nat. Biotechnol. 2004, 22, 435-439.

72. Wu, H.K.; Wheeler, A.; Zare, R.N. Chemical cytometry on a picoliter-scale integrated microfluidic chip. Proc. Natl. Acad. Sci. USA 2004, 101, 12809-12813.

73. Walch, A.; Specht, K.; Smida, J.; Aubele, M.; Zitzelsberger, H.; Hofler, H.; Werner, M. Tissue microdissection techniques in quantitative genome and gene expression analyses. Histochem. Cell Biol. 2001, 115, 269-276.

74. Guo, G.J.; Huss, M.; Tong, G.Q.; Wang, C.Y.; Sun, L.L.; Clarke, N.D.; Robson, P. Resolution of Cell Fate Decisions Revealed by Single-Cell Gene Expression Analysis from Zygote to Blastocyst. Dev. Cell 2010, 18, 675-685.

75. Hodne, K.; Haug, T.M.; Weltzien, F.A. Single-cell qPCR on dispersed primary pituitary cells-An optimized protocol. BMC Mol. Biol. 2010, 11, 82. doi:10.1186/1471-2199-11-82.

76. Citri, A.; Pang, Z.P.P.; Sudhof, T.C.; Wernig, M.; Malenka, R.C. Comprehensive qPCR profiling of gene expression in single neuronal cells. Nat. Protoc. 2012, 7, 118-127.

77. Brown, R.B.; Audet, J. Current techniques for single-cell lysis. J. R. Soc. Interface 2008, 5 , S131-S138. 
78. Svec, D.; Andersson, D.; Pekny, M.; Sjoback, R.; Kubista, M.; Stahlberg, A. Direct cell lysis for single-cell gene expression profiling. Front. Oncol. 2013, 3, 274, doi:10.3389/fonc.2013.00274.

79. Stahlberg, A.; Kubista, M. The workflow of single-cell expression profiling using quantitative real-time PCR. Expert Rev. Mol. Diagn. 2014, 14, 323-331.

80. Vonhippel, P.H.; Wong, K.Y. Neutral salts-Generality of their effects on stability of macromolecular conformations. Science 1964, 145, 577-580.

81. Nozaki, Y.; Tanford, C. Solubility of amino acids, diglycine, and triglycine in aqueous guanidine hydrochloride solutions. J. Biol. Chem. 1970, 245, 1648-1652.

82. Gordon, J.A. Denaturation of globular proteins. Interaction of guanidinium salts with three proteins. Biochemistry 1972, 11, 1862-1870.

83. Bengtsson, M.; Hemberg, M.; Rorsman, P.; Stahlberg, A. Quantification of mRNA in single cells and modelling of RT-qPCR induced noise. BMC Mol. Biol. 2008, 9, 11, doi:10.1186/1471-2199-9-63.

84. Geselowitz, D.A.; Neckers, L.M. Bovine serum-albumin is a major oligonucleotide-binding protein found on the surface of cultured-cells. Antisense Res. Dev. 1995, 5, 213-217.

85. Kreader, C.A. Relief of amplification inhibition in PCR with bovine serum albumin or T4 gene 32 protein. Appl. Environ. Microbiol. 1996, 62, 1102-1106.

86. Wilson, I.G. Inhibition and facilitation of nucleic acid amplification. Appl. Environ. Microbiol. 1997, 63, 3741-3751.

87. Abu Al-Soud, W.; Radstrom, P. Effects of amplification facilitators on diagnostic PCR in the presence of blood, feces, and meat. J. Clin. Microbiol. 2000, 38, 4463-4470.

88. Arnedo, A.; Espuelas, S.; Irache, J.M. Albumin nanoparticles as carriers for a phosphodiester oligonucleotide. Int. J. Pharm. 2002, 244, 59-72.

89. Farell, E.M.; Alexandre, G. Bovine serum albumin further enhances the effects of organic solvents on increased yield of polymerase chain reaction of GC-rich templates. BMC Res. Notes 2012, 5, 257.

90. Deprez, R.H.L.; Fijnvandraat, A.C.; Ruijter, J.M.; Moorman, A.F.M. Sensitivity and accuracy of quantitative real-time polymerase chain reaction using SYBR green I depends on cDNA synthesis conditions. Anal. Biochem. 2002, 307, 63-69.

91. Stahlberg, A.; Hakansson, J.; Xian, X.J.; Semb, H.; Kubista, M. Properties of the reverse transcription reaction in mRNA quantification. Clin. Chem. 2004, 50, 509-515.

92. Islam, S.; Zeisel, A.; Joost, S.; la Manno, G.; Zajac, P.; Kasper, M.; Lonnerberg, P.; Linnarsson, S. Quantitative single-cell RNA-seq with unique molecular identifiers. Nat. Methods 2014, 11, 163-166.

93. Ikeda, R.A. The efficiency of promoter clearance distinguishes T7 class-II and class-III promoters. J. Biol. Chem. 1992, 267, 11322-11328.

94. Ikeda, R.A.; Lin, A.C.; Clarke, J. Initiation of transcription by T7-RNA polymerase at its natural promoters. J. Biol. Chem. 1992b, 267, 2640-2649. 
95. Pabon, C.; Modrusan, Z.; Ruvolo, M.V.; Coleman, I.M.; Daniel, S.; Yue, H.; Arnold, L.J.; Reynolds, M.A. Optimized T7 amplification system for microarray analysis. Biotechniques 2001, 31, 874-879.

96. Wang, J.; Hu, L.; Hamilton, S.R.; Coombes, K.R.; Zhang, W. RNA amplification strategies for cDNA microarray experiments. BioTechniques 2003, 34, 394-400.

97. Moll, P.R.; Duschl, J.; Richter, K. Optimized RNA amplification using T7-RNA-polymerase based in vitro transcription. Anal. Biochem. 2004, 334, 164-174.

98. Brady, G.; Billia, F.; Knox, J.; Hoang, T.; Kirsch, I.R.; Voura, E.B.; Hawley, R.G.; Cumming, R.; Buchwald, M.; Siminovitch, K.; et al. analysis of gene-expression in a complex differentiation hierarchy by global amplification of cDNA from single cells. Curr. Biol. 1995, 5, 909-922.

99. Iscove, N.N.; Barbara, M.; Gu, M.; Gibson, M.; Modi, C.; Winegarden, N. Representation is faithfully preserved in global cDNA amplified exponentially from sub-picogram quantities of mRNA. Nat. Biotechnol. 2002, 20, 940-943.

100. Subkhankulova, T.; Livesey, F.J. Comparative evaluation of linear and exponential amplification techniques for expression profiling at the single-cell level. Genome Biol. 2006, 7, 16, doi:10.1186/gb-2006-7-3-r18.

101. Lang, J.E.; Magbanua, M.J.M.; Scott, J.H.; Makrigiorgos, G.M.; Wang, G.; Federman, S.; Esserman, L.J.; Park, J.W.; Haqq, C.M. A comparison of RNA amplification techniques at sub-nanogram input concentration. Bmc Genom. 2009, 10, 12, doi:10.1186/1471-2164-10326.

102. Zhu, Y.Y.; Machleder, E.M.; Chenchik, A.; Li, R.; Siebert, P.D. Reverse transcriptase template switching: A SMART ${ }^{\mathrm{TM}}$ approach for full-length cDNA library construction. Biotechniques 2001, 30, 892-897.

103. Tang, F.C.; Barbacioru, C.; Wang, Y.Z.; Nordman, E.; Lee, C.; Xu, N.L.; Wang, X.H.; Bodeau, J.; Tuch, B.B.; Siddiqui, A.; et al. mRNA-Seq whole-transcriptome analysis of a single cell. Nat. Methods 2009, 6, 377-382.

104. Islam, S.; Kjallquist, U.; Moliner, A.; Zajac, P.; Fan, J.B.; Lonnerberg, P.; Linnarsson, S. Characterization of the single-cell transcriptional landscape by highly multiplex RNA-seq. Genome Res. 2011, 21, 1160-1167.

105. Hashimshony, T.; Wagner, F.; Sher, N.; Yanai, I. CEL-Seq: Single-cell RNA-seq by multiplexed linear amplification. Cell Rep. 2012, 2, 666-673.

106. Ramskold, D.; Luo, S.J.; Wang, Y.C.; Li, R.; Deng, Q.L.; Faridani, O.R.; Daniels, G.A.; Khrebtukova, I.; Loring, J.F.; Laurent, L.C.; et al. Full-length mRNA-Seq from single-cell levels of RNA and individual circulating tumor cells. Nat. Biotechnol. 2012, 30, 777-782.

107. Sasagawa, Y.; Nikaido, I.; Hayashi, T.; Danno, H.; Uno, K.D.; Imai, T.; Ueda, H.R. Quartz-Seq: A highly reproducible and sensitive single-cell RNA sequencing method, reveals non-genetic gene-expression heterogeneity. Genome Biol. 2013, 14, 17, doi:10.1186/gb-2013-14-4-r31.

108. Koch, W.H. Technology platforms for pharmacogenomic diagnostic assays. Nat. Rev. Drug Discov. 2004, 3, 749-761. 
109. Persson, K.; Hamby, K.; Ugozzoli, L.A. Four-color multiplex reverse transcription polymerase chain reaction - Overcoming its limitations. Anal. Biochem. 2005, 344, 33-42.

110. Primer3plus. Available online: http://www.bioinformatics.nl/cgi-bin/primer3plus/ primer3plus.cgi (accessed on 5 November 2015).

111. Vector NTI. Available online: http:/www.lifetechnologies.com/no/en/home/life-science/ cloning/vector-nti-software.html (accessed on 5 November 2015).

112. Chandler, D.P.; Wagnon, C.A.; Bolton, H. Reverse transcriptase (RT) inhibition of PCR at low concentrations of template and its implications for quantitative RT-PCR. Appl. Environ. Microbiol. 1998, 64, 669-677.

113. Liss, B. Improved quantitative real-time RT-PCR for expression profiling of individual cells. Nucleic Acids Res. 2002, 30, 9, doi:10.1021/nn800851d.

114. Nolan, T.; Hands, R.E.; Ogunkolade, W.; Bustin, S.A. SPUD: A quantitative PCR assay for the detection of inhibitors in nucleic acid preparations. Anal. Biochem. 2006, 351, 308-310.

115. Singhal, R.; Orynbayeva, Z.; Sundaram, R.V.K.; Niu, J.J.; Bhattacharyya, S.; Vitol, E.A.; Schrlau, M.G.; Papazoglou, E.S.; Friedman, G.; Gogotsi, Y. Multifunctional carbon-nanotube cellular endoscopes. Nat. Nanotechnol. 2011, 6, 57-64.

116. Schrlau, M.G.; Dun, N.J.; Bau, H.H. Cell electrophysiology with carbon nanopipettes. ACS Nano 2009, 3, 563-568. 


\title{
Digital Microfluidics for Manipulation and Analysis of a Single Cell
}

\section{Jie-Long He, An-Te Chen, Jyong-Huei Lee and Shih-Kang Fan}

\begin{abstract}
The basic structural and functional unit of a living organism is a single cell. To understand the variability and to improve the biomedical requirement of a single cell, its analysis has become a key technique in biological and biomedical research. With a physical boundary of microchannels and microstructures, single cells are efficiently captured and analyzed, whereas electric forces sort and position single cells. Various microfluidic techniques have been exploited to manipulate single cells through hydrodynamic and electric forces. Digital microfluidics (DMF), the manipulation of individual droplets holding minute reagents and cells of interest by electric forces, has received more attention recently. Because of ease of fabrication, compactness and prospective automation, DMF has become a powerful approach for biological application. We review recent developments of various microfluidic chips for analysis of a single cell and for efficient genetic screening. In addition, perspectives to develop analysis of single cells based on DMF and emerging functionality with high throughput are discussed.
\end{abstract}

Reprinted from Int. J. Mol. Sci. Cite as: He, J.-L.; Chen, A.-T.; Lee, J.-H.; Fan, S.-K. Digital Microfluidics for Manipulation and Analysis of a Single Cell. Int. J. Mol. Sci. 2015, 16, 22319-22332.

\section{Introduction}

Multicellular organisms are composed of varied cells grouped into specialized tissues and organs, which typically comprise cells of diverse types present in widely varying abundance. The single cell is the basic structural and functional unit of a living organism. The most critical knowledge for biological and biomedical science is constructed from research on cell biology [1,2]; this information about cell functionality and behavior has been applied in many clinical and biomedical applications [3], such as drug development, disease diagnosis, cancer research and assisted reproductive technology (ART).

Traditional cell assays to study cell differentiation, gene expression and drug response were focused mainly on a population of cells. For an average result of multiple cells, the outcome of all cells is assumed to be homogeneous, but several authors have found cellular heterogeneity or multi-modal distributions in a cell population [4-6]. The heterogeneity might arise through genetic or non-genetic processes, which induce distinct cellular decision-making [7]. If an average response of multiple cells is taken to be representative of a typical population, cellular heterogeneity might result in a misleading interpretation [8]. Analysis of a single cell indicated that individual cells that form a cell population might have a complicated distribution. This information is ignored in a traditional cell assay, which emphasizes evaluating the mean of a cell population [9]. To understand the variability from cell to cell and to improve the clinical and biomedical applicability, development of new approaches to isolate, to manipulate, to treat and to analyze 
single cells are required. Analysis of single cells has become a key technique in biological and biomedical research, as one can thereby analyze individual cells within a cell population $[1,7]$.

Various techniques for analysis of a single cell have been developed, such as flow cytometry and microfluidic chips [10]. Flow cytometry, which has been under development for many years, has become a mature technique for single-cell analysis. In particular, fluorescence-activated cell sorting (FACS), builds upon flow cytometry in order to sort single cells that are tagged with specific fluorescent markers $[11,12]$. Flow cytometry is, however, a complicated and costly system: it cannot support analysis of cells in real time in their natural environment. Furthermore, integration of an entire assay based on a single cell typically entails processes such as cell manipulation, treatment and final detection. To overcome these problems, integrating various methods of cell manipulation with microfluidic lab-on-a-chip (LOC) platforms, also known as microfluidic chips, has become a major activity in assays of single cells [8,11,13-15]. Microfluidic chips could provide efficient genetic screening through a well-controlled microenvironment for analysis and treatment of a single cell. On these platforms, isolation of a single cell, purification of mRNA and subsequent multiplex quantitative polymerase chain reaction (qPCR) in real time must be effected on a chip [16]. Single-cell genomics, transcriptomics, epigenomics and proteomics will allow many enduring questions in biological and biomedical sciences to be answered [17-20]. Digital microfluidics (DMF), the manipulation of individual droplets by electric forces, is one of the particularly important techniques for single cell manipulation and analysis on a microfluidic chip. In this paper, we review recent developments in DMF chips for analysis of a single cell and efficient genetic screening, which involve manipulation of a single cell, next-generation sequencing (NGS) and assisted-reproductive applications. We conclude with views on the future development of DMF chips for analysis of a single cell and discuss how this emerging efficient tool can advance biological and biomedical research.

\section{Microfluidic Chips for Analysis of a Single Cell}

In an organism, a single cell is small: its volume is about $1 \mathrm{pL}$. Cells constituting a tissue or organ are complicated and diverse. An extracellular matrix (ECM) provides structural and biochemical support of the structural connection. To analyze a single cell on a chip, a microfluidic chip typically entails integration of four functions: (1) cell sorting, through microwells [21-25], traps [26-29], optical tweezers [30-33] or dielectrophoresis (DEP) [34-37] to isolate a single cell; (2) cell manipulation, through a traditional syringe pump [38] or an electrowetting-on-dielectric (EWOD) technique [39]; (3) cell lysis, through a mechanical [40], chemical [24] or electrical [23,41,42] process; and (4) analysis of an individual cell, such as with PCR [21,43] or NGS [44].

Microfluidic chips have been applied in biological and biomedical research, including culturing, sorting, patterning and genetic screening of single cells for clinical diagnostics [45-49]. These platforms have become a promising tool for efficient genetic screening through analysis of single cells, because these microfluidic chips can provide rapid, real-time and automated analysis. Gossett et al. [50] demonstrated an automated microfluidic technique capable of probing single cells. A rapid assay of the deformability of native populations of leukocytes and malignant cells in pleural effusions has been enabled on this chip. Guan et al. [51] introduced a new microfluidic chip 
with real-time feedback control to evaluate single-cell deformability, which was used to discriminate different kinds of cells for cancer diagnosis [30]. Guo et al. [52] produced a microfluidic chip to distinguish red blood cells containing parasitic Plasmodium falciparum from uninfected cells. Several microfluidic chips have been generated to capture single cells and to measure the impedance of the cells, such as human cervical epithelioid carcinoma (HeLa) cells $[53,54]$ or circulating tumor cells (CTCs) from blood [55,56]. Kurz et al. [57] reported a microfluidic chip to trap single cells and to measure the impedance for the monitoring of sub-toxic effects on cell membranes.

The method most frequently used to isolate a single cell is physical separation. At designed physical boundaries, an individual cell is isolated, captured and sorted with mechanical structures on a chip. Capturing an individual cell with microwells is an attractive strategy, because it is simple and easily operated. Jen et al. $[23,24]$ reported microfluidic chips with arrays of microwells that isolated individual cells and provided chemical and electric lysis of a single cell with high throughput (Figure 1a). Lindstrom et al. [21,22,58,59] developed a novel microplate with microwells for efficient analyses of single cells. This platform allowed each single cell to be cultivated and analyzed individually for reprogramming factor evaluation on stem cells [22], PCR amplification and genetic analysis [21] (Figure 1b).

Microfluidic hydrodynamic traps combine dynamic cell isolation with prospective high throughput on a chip [60,61]. Di Carlo et al. [26,62] produced a dynamic platform that allows culture of a single cell with a consistent environment and dynamic control of individual cells (Figure 2a). Kobel et al. [60] reported a microfluidic chip with efficiency of trapping a single cell enhanced up to $97 \%$ (Figure $2 b$ ).

Compared with use of mechanical structures, isolating a cell with an optical or electric force is a contact-free method that eliminates deformation and damage of cells [63,64]. Here we focus more on the electric forces, including DEP and EWOD. The DEP force generated with a non-uniform electric field interacting with polarized, suspended particles [65-67] has been widely used to manipulate cells $[39,63,68,69]$. The DEP force is classified as positive DEP (pDEP) and negative DEP (nDEP) based on the polarizability of the particles or cells and the liquid. Fan et al. [39] used DEP forces to concentrate suspended particles in a liquid droplet with dielectric-coated electrodes patterned on a plate (Figure 3a). Creating two droplets with mammalian cells and polystyrene beads at distinct concentrations was achieved with DEP and EWOD (Figure 3b). 
(a).

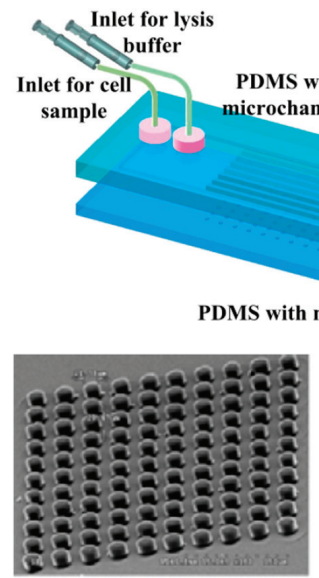

\section{SU-8 mold}

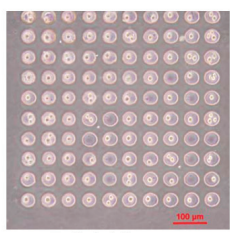

Bright image
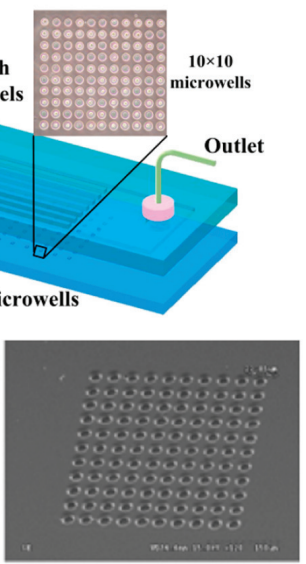

PDMS replica

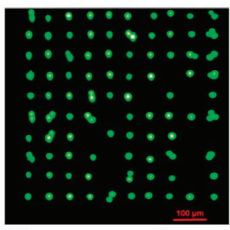

Fluorescence image

(b).
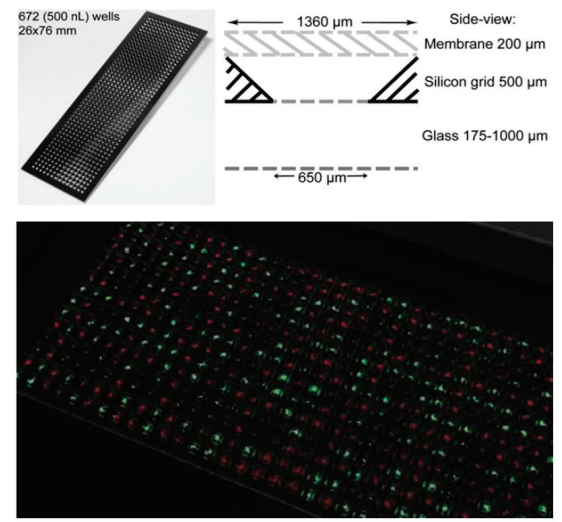

Cells seeding (conceptual sketch)

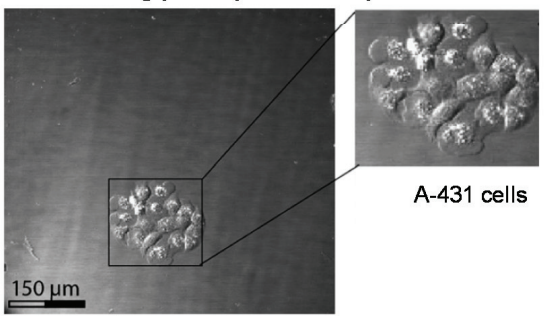

Figure 1. Individual cells isolated on a chip with microwells described in: (a) Jen et al., 2012 [24]; (b) Lindstrom et al., 2009 [21]. Reproduction of the figures has been made with permission from Multidisciplinary Digital Publishing Institute and The Royal Society of Chemistry.

\section{Digital Microfluidic Chips and Biological Application}

Digital microfluidics (DMF), according to which tiny droplets are manipulated with electric forces, including EWOD and DEP, have been confirmed to be a powerful platform for reagent addition, droplet transmission, solution mixing, splitting and dispensing for biological application [70-80]. For a simple configuration of device and easy modular interfaces, portable and wearable DMF systems with assembled modules for continuous actuation of droplets were demonstrated [70] (Figure 3c). 
(a).
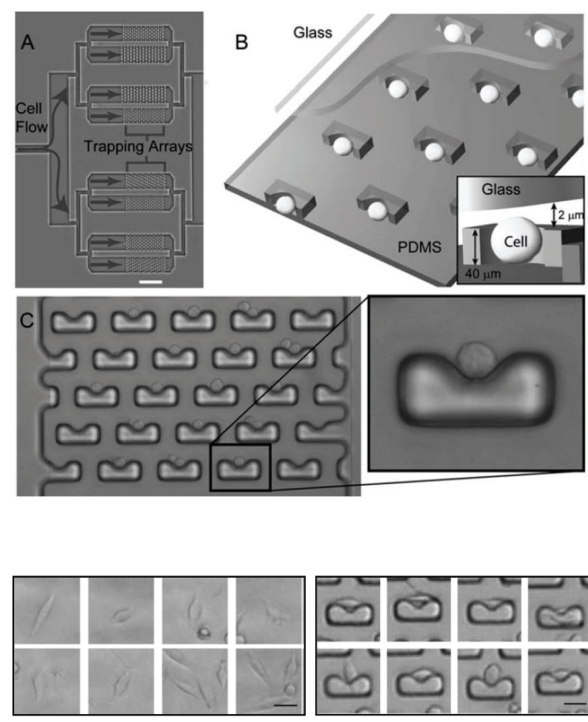

Glass substrate

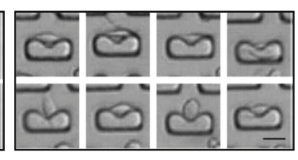

Trapping structures

(b).
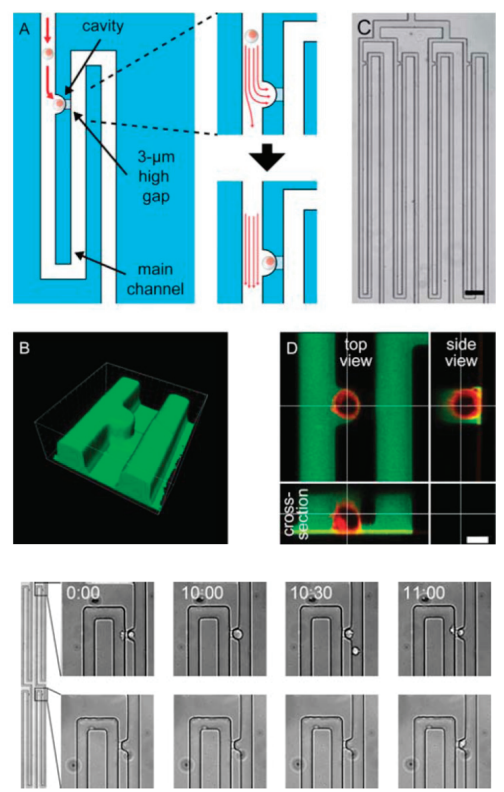

EG7 cells

Figure 2. Individual cell isolated on a chip with microfluidic hydrodynamic traps described in: (a) Di Carlo et al., 2006 [26]: (A) A photograph of the cell trapping device; (B) A diagram of the device and mechanism of trapping; (C) A high resolution micrograph of the trapping device; (b) Kobel et al., 2010 [60]: (A) Schematic illustration of the single cell trap; (B) A three-dimensional reconstruction image of the cell trap; (C) An array of the single cell traps; (D) An orthogonal view of a fluorescently labeled single cell. Reproduction of the figures has been made with permission from The Royal Society of Chemistry.

A DMF chip with arrays of electrodes is typically fabricated on indium tin oxide (ITO) glass plates using photolithography and wet etching. The patterned ITO electrodes are then covered with a dielectric and a hydrophobic layer $[16,39,73,81]$. The advantages of DMF include ease of fabrication, simple device structure, small consumption of reagents, easy integration with analytical instruments and prospective automation. Thus, DMF has become highly suitable for biological application. Barbulovic-Nad et al. [80] introduced a DMF chip to implement cell-based assays; the platform was demonstrated to be advantageous for cell-based assays because of potential for automated manipulation of multiple reagents. Vergauwe et al. [78] reported a DMF chip for homogeneous and heterogeneous bio-assays with great analytical performance capable of medical applications. Kumar et al. [75] demonstrated the first use of a DMF technique for individual protoplasts from Arabidopsis thaliana plants. Shih et al. developed the first DMF chip capable of cell impedance sensing [76]; they also integrated droplet-in-channel microfluidics with DMF to develop a novel chip to perform complicated assays [81]. 
(a).
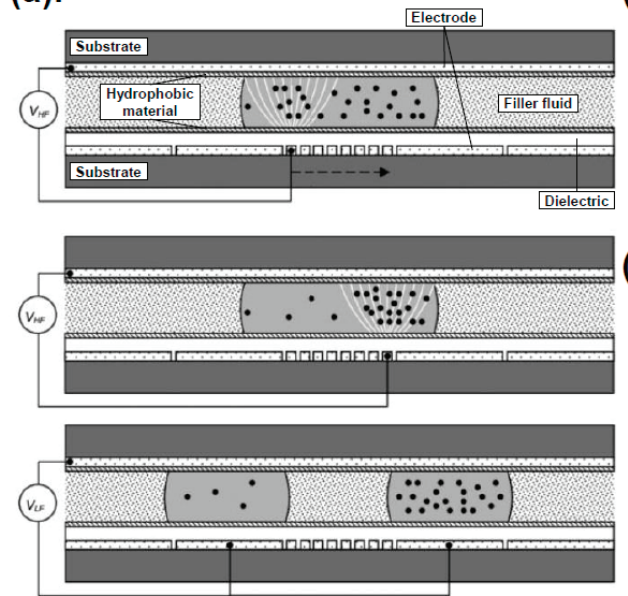

(d).

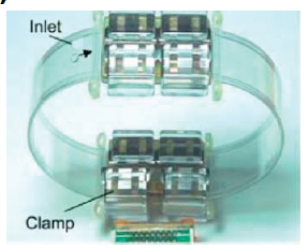

(b).

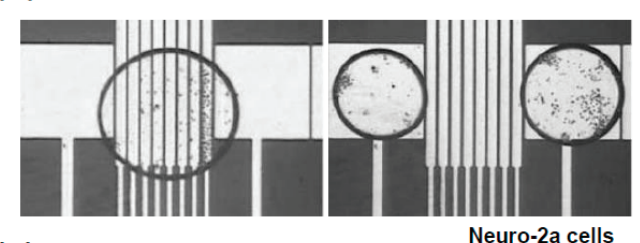

(c).
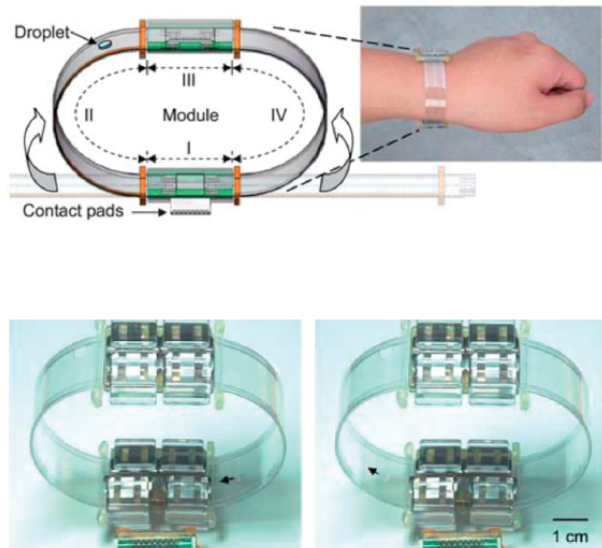

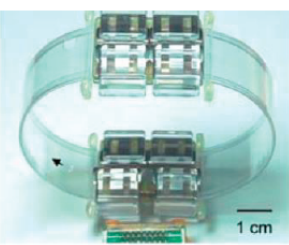

Figure 3. Dielectrophoresis (DEP) forces exerting on the suspended particles described by Fan et al., 2008 [39]. (a) A parallel-plate device with square and strip electrodes to manipulate droplets and a particle; (b) DEP forces exerted on suspended particles including mammalian cells (Neuro-2a); (c,d) Prototype of EWOD-based, continuously microfluidic module for a portable system reported by Fan et al., 2011 [70]. Reproduction of the figures has been made with permission from The Royal Society of Chemistry.

This work demonstrates that DMF chips would be a generic and powerful platform for the biological assays, including drug screening, immunoassays, analysis of single cells and digital PCR. This promising new technique might allow the efficient genetic screening based on a single cell to become a reality.

\section{Digital Microfluidic Chips for Genetic Screening}

Investigating gene expression and developing genetic screening at a level of a single cell provides an important capability to resolve the problem of disease etiology, cancer pathology and other biomedical applications [82]. Traditional methods of genetic screening require a large amount of sample for an analysis, which typically decreases the sensitivity and accuracy on analysis of only a single cell $[83,84]$. Various microfluidic techniques have been developed to address this problem. Digital polymerase chain reaction (digital PCR) platforms have measured DNA or cDNA of a single cell $[85,86]$, but challenges persist in treating the integration of varied programs for genetic analyses of a single cell on a device, including cell sorting, manipulation, lysis, PCR and genetic 
screening. Within the past decade, microfluidic chips have become one of the most powerful platforms to achieve efficient genetic screening at the level of a single cell $[16,87]$. Toriello et al. [88] and Bontoux et al. [89] reported a polydimethylsiloxane (PDMS) device to analyze the gene expression of single cells, and Marcus et al. [90] developed a microfluidic chip with integrated flow that conducted cell capture, lysis, mRNA purification, cDNA synthesis and purification but with a complicated auxiliary system for control.

As mentioned above, the DMF chip has advantages of ease of fabrication, simple supporting instrumentation and prospective automation. Rival et al. [16] described an integrated and automated EWOD system to perform a complete workflow from the isolation of a single cell to a genetic analysis (Figure 4). DMF is becoming a powerful approach for biological applications, even enabling sample preparation for PCR to develop an efficient genetic screening platform based on a single cell $[16,91,92]$.

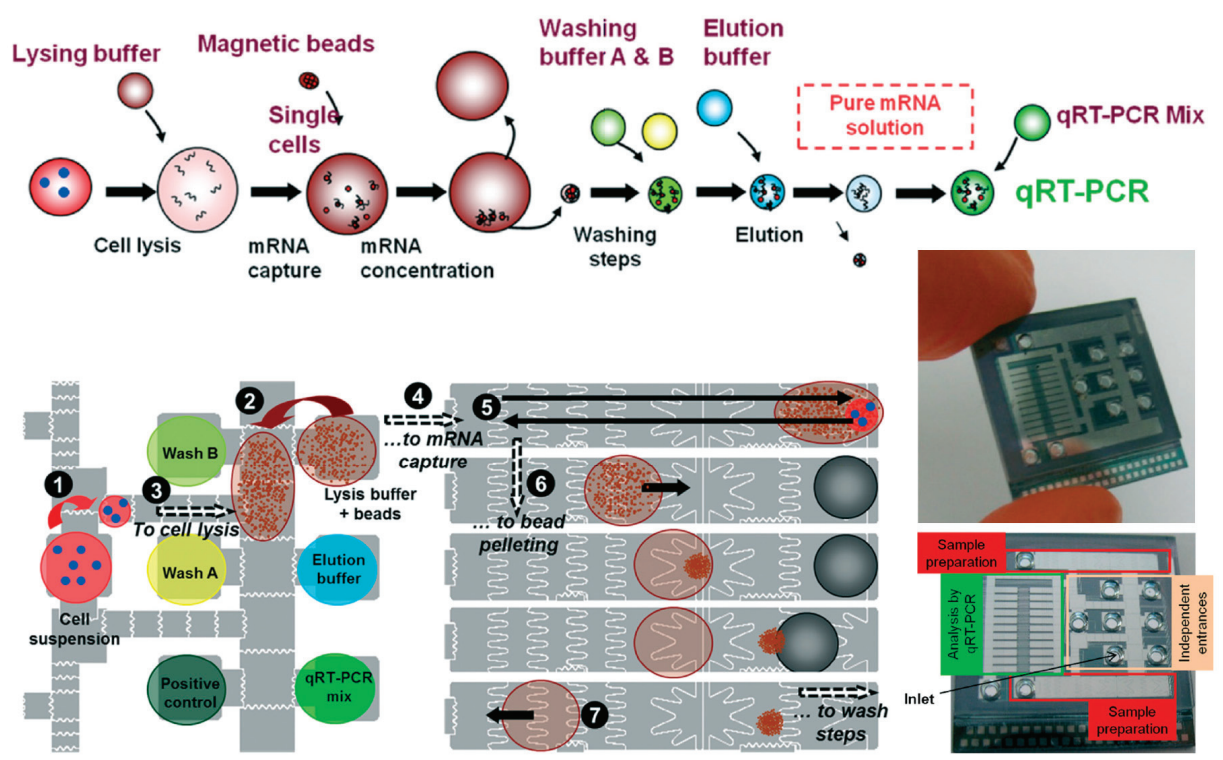

Figure 4. An integrated EWOD system for genetic analysis based on a single cell described by Rival et al., 2014 [16]. The lysis and mRNA capture steps: (1) A droplet containing a few cells is dispensed; (2) A droplet of lysis buffer and magnetic beads is dispensed; (3) A droplet with the cells and a droplet with the beads are merged for cell lysis; (4) This merged droplet is moved to the "sample preparation" electrodes for operating magnetic beads; (5) The droplet is moved back and forth to enable bead mixing and mRNA capture; (6) The droplet is moved towards the magnet, the beads are concentrated to result in bead extraction; (7) An empty droplet is moved towards the waist. Reproduction of the figures has been made with permission from The Royal Society of Chemistry. 


\section{Efficient Genetic Screening for Assisted Reproductive Techniques}

The early development of a mammalian embryo is a complicated process involving an upheaval of a transcriptional architecture [93-95]. In applications, human in vitro fertilization (IVF) is an important scientific achievement in the twentieth century, but until recently there has been little knowledge of regulatory mechanisms in genes of early mammalian embryos. The early embryo undergoes cleavage divisions in a series from two cell, four cells, eight cells, morula, even to blastocyst. A platform for genetic screening based on a single cell could provide critical knowledge to clarify regulatory mechanisms of genes in early mammalian embryos [93]. This emerging efficient technique would benefit a biomedical approach, such as assisted reproductive technology (ART).

The microfluidic ART platforms under development are focused on simulation of the Fallopian tube to optimize the IVF, especially the early embryo culture in vitro [96-98]. Pre-implantation genetic diagnosis (PGD) has been recently developed to detect genetic diseases $[99,100]$. The current methods of sorting single cells include taking trophectoderm cells from blastocyst, blastomeres from embryos at a cleavage stage and polar bodies from the oocyte or zygote [101]. The advantages of a DMF chip include simple accompanying instrumentation and prospective automation for sorting single cells from an early embryo. Huang et al. [98] demonstrated a EWOD-based microfluidic device for culture of early mammalian embryos in vitro, presaging future clinical application. Although a DMF chip applied in efficient genetic diagnosis is still at an initial stage, we believe that this powerful platform will have a major impact on the ART field.

\section{Conclusions}

Recent developments of microfluidic chips for single cell analysis were reviewed; microfluidic techniques provide numerous advantages for biological and biomedical research, including ease for modularity, small sample requirement, potential of automation, and high-throughput. Perspectives on DMF in analysis of a single cell and efficient genetic screening were particularly focused on and described. We expect that these novel single-cell techniques on microfluidic chips will be important in biomedical areas.

\section{Acknowledgments}

Jie-Long He thanks the Ministry of Science and Technology, Taiwan for providing a post-doctoral fellowship.

\section{Author Contributions}

Jie-Long He and Shih-Kang Fan wrote the manuscript. An-Te Chen and Jyong-Huei Lee assisted in collecting and integrating the references.

\section{Conflicts of Interest}

The authors declare no conflict of interest. 


\section{References}

1. Svahn, H.A.; van den Berg, A. Single cells or large populations? Lab Chip 2007, 7, 544-546.

2. Bahcall, O.G. Single cell resolution in regulation of gene expression. Mol. Syst. Biol. 2005, 1, 2005.0015, doi:10.1038/msb4100020.

3. Dittrich, P.S.; Tachikawa, K.; Manz, A. Micro total analysis systems. Latest advancements and trends. Anal. Chem. 2006, 78, 3887-3908.

4. Graf, T.; Stadtfeld, M. Heterogeneity of embryonic and adult stem cells. Cell Stem Cell 2008, 3, 480-483.

5. Zhou, L.; Shen, Y.; Jiang, L.; Yin, D.; Guo, J.; Zheng, H.; Sun, H.; Wu, R.; Guo, Y. Systems mapping for hematopoietic progenitor cell heterogeneity. PLoS ONE 2015, 10, e0126937.

6. Donati, G.; Watt, F.M. Stem cell heterogeneity and plasticity in epithelia. Cell Stem Cell 2015, 16, 465-476.

7. Perkins, T.J.; Swain, P.S. Strategies for cellular decision-making. Mol. Syst. Biol. 2009, $5,326$.

8. Yin, H.; Marshall, D. Microfluidics for single cell analysis. Curr. Opin. Biotechnol. 2012, 23, 110-119.

9. Wang, D.; Bodovitz, S. Single cell analysis: The new frontier in "omics". Trends Biotechnol. 2010, 28, 281-290.

10. Shah, P.; Zhu, X.; Chen, C.; Hu, Y.; Li, C.Z. Lab-on-chip device for single cell trapping and analysis. Biomed. Microdevices 2014, 16, 35-41.

11. Cheung, K.C.; di Berardino, M.; Schade-Kampmann, G.; Hebeisen, M.; Pierzchalski, A.; Bocsi, J.; Mittag, A.; Tarnok, A. Microfluidic impedance-based flow cytometry. Cytom. Part A J. Int. Soc. Anal. Cytol. 2010, 77, 648-666.

12. Ning, B.; Yihong, Z.; Chang, L. Microfluidic electroporative flow cytometry for studying single-cell biomechanics. Anal. Chem. 2008, 80, 7714-7719.

13. Shah, P.; Kaushik, A.; Zhu, X.; Zhang, C.; Li, C.Z. Chip based single cell analysis for nanotoxicity assessment. Analyst 2014, 139, 2088-2098.

14. Lindstrom, S.; Andersson-Svahn, H. Miniaturization of biological assays-Overview on microwell devices for single-cell analyses. Biochim. Biophys. Acta 2011, 1810, 308-316.

15. Le Gac, S.; van den Berg, A. Single cells as experimentation units in lab-on-a-chip devices. Trends Biotechnol. 2010, 28, 55-62.

16. Rival, A.; Jary, D.; Delattre, C.; Fouillet, Y.; Castellan, G.; Bellemin-Comte, A.; Gidrol, X. An EWOD-based microfluidic chip for single-cell isolation, mRNA purification and subsequent multiplex qPCR. Lab Chip 2014, 14, 3739-3749.

17. Shapiro, E.; Biezuner, T.; Linnarsson, S. Single-cell sequencing-based technologies will revolutionize whole-organism science. Nat. Rev. Genet. 2013, 14, 618-630.

18. Ullal, A.V.; Peterson, V.; Agasti, S.S.; Tuang, S.; Juric, D.; Castro, C.M.; Weissleder, R. Cancer cell profiling by barcoding allows multiplexed protein analysis in fine-needle aspirates. Sci. Transl. Med. 2014, 6, 219 ra219. 
19. Salehi-Reyhani, A.; Burgin, E.; Ces, O.; Willison, K.R.; Klug, D.R. Addressable droplet microarrays for single cell protein analysis. Analyst 2014, 139, 5367-5374.

20. Kalisky, T.; Quake, S.R. Single-cell genomics. Nat. Methods 2011, 8, 311-314.

21. Lindstrom, S.; Hammond, M.; Brismar, H.; Andersson-Svahn, H.; Ahmadian, A. PCR amplification and genetic analysis in a microwell cell culturing chip. Lab Chip 2009, 9, 3465-3471.

22. Lindstrom, S.; Eriksson, M.; Vazin, T.; Sandberg, J.; Lundeberg, J.; Frisen, J.; Andersson-Svahn, H. High-density microwell chip for culture and analysis of stem cells. PLoS ONE 2009, 4, e6997.

23. Jen, C.P.; Amstislavskaya, T.G.; Liu, Y.H.; Hsiao, J.H.; Chen, Y.H. Single-cell electric lysis on an electroosmotic-driven microfluidic chip with arrays of microwells. Sensors (Basel) 2012, 12, 6967-6977.

24. Jen, C.P.; Hsiao, J.H.; Maslov, N.A. Single-cell chemical lysis on microfluidic chips with arrays of microwells. Sensors (Basel) 2012, 12, 347-358.

25. Osada, K.; Hosokawa, M.; Yoshino, T.; Tanaka, T. Monitoring of cellular behaviors by microcavity array-based single-cell patterning. Analyst 2014, 139, 425-430.

26. Di Carlo, D.; Wu, L.Y.; Lee, L.P. Dynamic single cell culture array. Lab Chip 2006, 6, 1445-1449.

27. Skelley, A.M.; Kirak, O.; Suh, H.; Jaenisch, R.; Voldman, J. Microfluidic control of cell pairing and fusion. Nat. Methods 2009, 6, 147-152.

28. Lee, S.-W.; Kang, J.Y.; Lee, I.-H.; Ryu, S.-S.; Kwak, S.-M.; Shin, K.-S.; Kim, C.; Jung, H.-I.; Kim, T.-S. Single-cell assay on CD-like lab chip using centrifugal massive single-cell trap. Sens. Actuators A Phys. 2008, 143, 64-69.

29. Liu, W.; Dechev, N.; Foulds, I.G.; Burke, R.; Parameswaran, A.; Park, E.J. A novel permalloy based magnetic single cell micro array. Lab Chip 2009, 9, 2381-2390.

30. Remmerbach, T.W.; Wottawah, F.; Dietrich, J.; Lincoln, B.; Wittekind, C.; Guck, J. Oral cancer diagnosis by mechanical phenotyping. Cancer Res. 2009, 69, 1728-1732.

31. Guck, J.; Schinkinger, S.; Lincoln, B.; Wottawah, F.; Ebert, S.; Romeyke, M.; Lenz, D.; Erickson, H.M.; Ananthakrishnan, R.; Mitchell, D.; et al. Optical deformability as an inherent cell marker for testing malignant transformation and metastatic competence. Biophys. J. 2005, 88, 3689-3698.

32. Wang, M.M.; Tu, E.; Raymond, D.E.; Yang, J.M.; Zhang, H.; Hagen, N.; Dees, B.; Mercer, E.M.; Forster, A.H.; Kariv, I.; et al. Microfluidic sorting of mammalian cells by optical force switching. Nat. Biotechnol. 2005, 23, 83-87.

33. Yang, T.; Paie, P.; Nava, G.; Bragheri, F.; Martinez Vazquez, R.; Minzioni, P.; Veglione, M.; di Tano, M.; Mondello, C.; Osellame, R.; et al. An integrated optofluidic device for single-cell sorting driven by mechanical properties. Lab Chip 2015, 15, 1262-1266.

34. Taff, B.M.; Voldman, J. A scalable addressable positive-dielectrophoretic cell-sorting array. Anal. Chem. 2005, 77, 7976-7983. 
35. Hu, X.; Bessette, P.H.; Qian, J.; Meinhart, C.D.; Daugherty, P.S.; Soh, H.T. Marker-specific sorting of rare cells using dielectrophoresis. Proc. Natl. Acad. Sci. USA 2005, 102, 15757-15761.

36. Shah, G.J.; Ohta, A.T.; Chiou, E.P.; Wu, M.C.; Kim, C.J. EWOD-driven droplet microfluidic device integrated with optoelectronic tweezers as an automated platform for cellular isolation and analysis. Lab Chip 2009, 9, 1732-1739.

37. Park, S.; Wijethunga, P.A.; Moon, H.; Han, B. On-chip characterization of cryoprotective agent mixtures using an EWOD-based digital microfluidic device. Lab Chip 2011, 11, 2212-2221.

38. Rosenbluth, M.J.; Lam, W.A.; Fletcher, D.A. Analyzing cell mechanics in hematologic diseases with microfluidic biophysical flow cytometry. Lab Chip 2008, 8, 1062-1070.

39. Fan, S.K.; Huang, P.W.; Wang, T.T.; Peng, Y.H. Cross-scale electric manipulations of cells and droplets by frequency-modulated dielectrophoresis and electrowetting. Lab Chip 2008, 8, 1325-1331.

40. Di Carlo, D.; Jeong, K.H.; Lee, L.P. Reagentless mechanical cell lysis by nanoscale barbs in microchannels for sample preparation. Lab Chip 2003, 3, 287-291.

41. Zheng, S.; Lin, H.; Liu, J.Q.; Balic, M.; Datar, R.; Cote, R.J.; Tai, Y.C. Membrane microfilter device for selective capture, electrolysis and genomic analysis of human circulating tumor cells. J. Chromatogr. A 2007, 1162, 154-161.

42. Bao, N.; Kodippili, G.C.; Giger, K.M.; Fowler, V.M.; Low, P.S.; Lu, C. Single-cell electrical lysis of erythrocytes detects deficiencies in the cytoskeletal protein network. Lab Chip 2011, $11,3053-3056$.

43. Ginsberg, S.D. RNA amplification strategies for small sample populations. Methods 2005, 37, 229-237.

44. Feng, X.; Du, W.; Luo, Q.; Liu, B.F. Microfluidic chip: Next-generation platform for systems biology. Anal. Chim. Acta 2009, 650, 83-97.

45. Koster, S.; Angile, F.E.; Duan, H.; Agresti, J.J.; Wintner, A.; Schmitz, C.; Rowat, A.C.; Merten, C.A.; Pisignano, D.; Griffiths, A.D.; et al. Drop-based microfluidic devices for encapsulation of single cells. Lab Chip 2008, 8, 1110-1115.

46. Clausell-Tormos, J.; Lieber, D.; Baret, J.C.; El-Harrak, A.; Miller, O.J.; Frenz, L.; Blouwolff, J.; Humphry, K.J.; Koster, S.; Duan, H.; et al. Droplet-based microfluidic platforms for the encapsulation and screening of mammalian cells and multicellular organisms. Chem. Biol. 2008, 15, 427-437.

47. Brouzes, E.; Medkova, M.; Savenelli, N.; Marran, D.; Twardowski, M.; Hutchison, J.B.; Rothberg, J.M.; Link, D.R.; Perrimon, N.; Samuels, M.L. Droplet microfluidic technology for single-cell high-throughput screening. Proc. Natl. Acad. Sci. USA 2009, 106, 14195-14200.

48. Yu, L.; Chen, M.C.; Cheung, K.C. Droplet-based microfluidic system for multicellular tumor spheroid formation and anticancer drug testing. Lab Chip 2010, 10, 2424-2432.

49. Pekin, D.; Skhiri, Y.; Baret, J.C.; le Corre, D.; Mazutis, L.; Salem, C.B.; Millot, F.; El Harrak, A.; Hutchison, J.B.; Larson, J.W.; et al. Quantitative and sensitive detection of rare mutations using droplet-based microfluidics. Lab Chip 2011, 11, 2156-2166. 
50. Gossett, D.R.; Tse, H.T.; Lee, S.A.; Ying, Y.; Lindgren, A.G.; Yang, O.O.; Rao, J.; Clark, A.T.; di Carlo, D. Hydrodynamic stretching of single cells for large population mechanical phenotyping. Proc. Natl. Acad. Sci. USA 2012, 109, 7630-7635.

51. Guan, G.; Chen, P.C.Y.; Peng, W.K.; Bhagat, A.A.; Ong, C.J.; Han, J. Real-time control of a microfluidic channel for size-independent deformability cytometry. J. Micromech. Microeng. 2012, 22, 105037.

52. Guo, Q.; Reiling, S.J.; Rohrbach, P.; Ma, H. Microfluidic biomechanical assay for red blood cells parasitized by Plasmodium falciparum. Lab Chip 2012, 12, 1143-1150.

53. Jang, L.S.; Wang, M.H. Microfluidic device for cell capture and impedance measurement. Biomed. Microdevices 2007, 9, 737-743.

54. Malleo, D.; Nevill, J.T.; Lee, L.P.; Morgan, H. Continuous differential impedance spectroscopy of single cells. Microfluid. Nanofluid. 2010, 9, 191-198.

55. Han, K.H.; Han, A.; Frazier, A.B. Microsystems for isolation and electrophysiological analysis of breast cancer cells from blood. Biosens. Bioelectron. 2006, 21, 1907-1914.

56. Chung, J.; Issadore, D.; Ullal, A.; Lee, K.; Weissleder, R.; Lee, H. Rare cell isolation and profiling on a hybrid magnetic/size-sorting chip. Biomicrofluidics 2013, 7, 54107.

57. Kurz, C.M.; Buth, H.; Sossalla, A.; Vermeersch, V.; Toncheva, V.; Dubruel, P.; Schacht, E.; Thielecke, H. Chip-based impedance measurement on single cells for monitoring sub-toxic effects on cell membranes. Biosens. Bioelectron. 2011, 26, 3405-3412.

58. Lindstrom, S.; Mori, K.; Ohashi, T.; Andersson-Svahn, H. A microwell array device with integrated microfluidic components for enhanced single-cell analysis. Electrophoresis 2009, 30, 4166-4171.

59. Lindstrom, S.; Larsson, R.; Svahn, H.A. Towards high-throughput single cell/clone cultivation and analysis. Electrophoresis 2008, 29, 1219-1227.

60. Kobel, S.; Valero, A.; Latt, J.; Renaud, P.; Lutolf, M. Optimization of microfluidic single cell trapping for long-term on-chip culture. Lab Chip 2010, 10, 857-863.

61. Bow, H.; Pivkin, I.V.; Diez-Silva, M.; Goldfless, S.J.; Dao, M.; Niles, J.C.; Suresh, S.; Han, J. A microfabricated deformability-based flow cytometer with application to malaria. Lab Chip 2011, 11, 1065-1073.

62. Di Carlo, D.; Lee, L.P. Dynamic single-cell analysis for quantitative biology. Anal. Chem. 2006, 78, 7918-7925.

63. Lan, K.C.; Jang, L.S. Integration of single-cell trapping and impedance measurement utilizing microwell electrodes. Biosens. Bioelectron. 2011, 26, 2025-2031.

64. Huang, N.T.; Zhang, H.L.; Chung, M.T.; Seo, J.H.; Kurabayashi, K. Recent advancements in optofluidics-based single-cell analysis: Optical on-chip cellular manipulation, treatment, and property detection. Lab Chip 2014, 14, 1230-1245.

65. Rahman, A.R.A.; Price, D.T.; Bhansali, S. Effect of electrode geometry on the impedance evaluation of tissue and cell culture. Sens. Actuators B Chem. 2007, 127, 89-96.

66. Fan, S.K.; Chiu, C.P.; Hsu, C.H.; Chen, S.C.; Huang, L.L.; Lin, Y.H.; Fang, W.F.; Chen, J.K.; Yang, J.T. Particle chain display-An optofluidic electronic paper. Lab Chip 2012, 12, $4870-4876$. 
67. Fan, S.K.; Chiu, C.P.; Huang, P.W. Transmittance tuning by particle chain polarization in electrowetting-driven droplets. Biomicrofluidics 2010, 4, 43011.

68. Choi, H.; Kim, K.B.; Jeon, C.S.; Hwang, I.; Lee, S.; Kim, H.K.; Kim, H.C.; Chung, T.D. A label-free DC impedance-based microcytometer for circulating rare cancer cell counting. Lab Chip 2013, 13, 970-977.

69. Mernier, G.; Duqi, E.; Renaud, P. Characterization of a novel impedance cytometer design and its integration with lateral focusing by dielectrophoresis. Lab Chip 2012, 12, 4344-4349.

70. Fan, S.K.; Yang, H.; Hsu, W. Droplet-on-a-wristband: Chip-to-chip digital microfluidic interfaces between replaceable and flexible electrowetting modules. Lab Chip 2011, 11, 343-347.

71. Fan, S.K.; Hsieh, T.H.; Lin, D.Y. General digital microfluidic platform manipulating dielectric and conductive droplets by dielectrophoresis and electrowetting. Lab Chip 2009, 9, 1236-1242.

72. Fan, S.K.; Chen, W.J.; Lin, T.H.; Wang, T.T.; Lin, Y.C. Reconfigurable liquid pumping in electric-field-defined virtual microchannels by dielectrophoresis. Lab Chip 2009, 9, 1590-1595.

73. Fan, S.K.; Yang, H.; Wang, T.T.; Hsu, W. Asymmetric electrowetting-Moving droplets by a square wave. Lab Chip 2007, 7, 1330-1335.

74. Choi, K.; Ng, A.H.; Fobel, R.; Wheeler, A.R. Digital microfluidics. Annu. Rev. Anal. Chem. 2012, 5, 413-440.

75. Kumar, P.T.; Toffalini, F.; Witters, D.; Vermeir, S.; Rolland, F.; Hertog, M.L.A.T.M.; Nicolai, B.M.; Puers, R.; Geeraerd, A.; Lammertyn, J. Digital microfluidic chip technology for water permeability measurements on single isolated plant protoplasts. Sens. Actuators B Chem. 2014, 199, 479-487.

76. Shih, S.C.; Barbulovic-Nad, I.; Yang, X.; Fobel, R.; Wheeler, A.R. Digital microfluidics with impedance sensing for integrated cell culture and analysis. Biosens. Bioelectron. 2013, 42, $314-320$.

77. Witters, D.; Vergauwe, N.; Vermeir, S.; Ceyssens, F.; Liekens, S.; Puers, R.; Lammertyn, J. Biofunctionalization of electrowetting-on-dielectric digital microfluidic chips for miniaturized cell-based applications. Lab Chip 2011, 11, 2790-2794.

78. Vergauwe, N.; Witters, D.; Ceyssens, F.; Vermeir, S.; Verbruggen, B.; Puers, R.; Lammertyn, J. A versatile electrowetting-based digital microfluidic platform for quantitative homogeneous and heterogeneous bio-assays. J. Micromech. Microeng. 2011, 21, 054026.

79. Vergauwe, N.; Witters, D.; Atalay, Y.T.; Verbruggen, B.; Vermeir, S.; Ceyssens, F.; Puers, R.; Lammertyn, J. Controlling droplet size variability of a digital lab-on-a-chip for improved bio-assay performance. Microfluid. Nanofluid. 2011, 11, 25-34.

80. Barbulovic-Nad, I.; Yang, H.; Park, P.S.; Wheeler, A.R. Digital microfluidics for cell-based assays. Lab Chip 2008, 8, 519-526.

81. Shih, S.C.; Gach, P.C.; Sustarich, J.; Simmons, B.A.; Adams, P.D.; Singh, S.; Singh, A.K. A droplet-to-digital (D2D) microfluidic device for single cell assays. Lab Chip 2015, 15, $225-236$. 
82. Thompson, A.M.; Gansen, A.; Paguirigan, A.L.; Kreutz, J.E.; Radich, J.P.; Chiu, D.T. Self-digitization microfluidic chip for absolute quantification of mRNA in single cells. Anal. Chem. 2014, 86, 12308-12314.

83. Thompson, A.M.; Paguirigan, A.L.; Kreutz, J.E.; Radich, J.P.; Chiu, D.T. Microfluidics for single-cell genetic analysis. Lab Chip 2014, 14, 3135-3142.

84. Lee, T.M.; Hsing, I.M. DNA-based bioanalytical microsystems for handheld device applications. Anal. Chim. Acta 2006, 556, 26-37.

85. Sanders, R.; Huggett, J.F.; Bushell, C.A.; Cowen, S.; Scott, D.J.; Foy, C.A. Evaluation of digital PCR for absolute DNA quantification. Anal. Chem. 2011, 83, 6474-6484.

86. White, R.A., 3rd; Quake, S.R.; Curr, K. Digital PCR provides absolute quantitation of viral load for an occult RNA virus. J. Virol. Methods 2012, 179, 45-50.

87. Bennett, M.R.; Hasty, J. Microfluidic devices for measuring gene network dynamics in single cells. Nat. Rev. Genet. 2009, 10, 628-638.

88. Toriello, N.M.; Douglas, E.S.; Thaitrong, N.; Hsiao, S.C.; Francis, M.B.; Bertozzi, C.R.; Mathies, R.A. Integrated microfluidic bioprocessor for single-cell gene expression analysis. Proc. Natl. Acad. Sci. USA 2008, 105, 20173-20178.

89. Bontoux, N.; Dauphinot, L.; Vitalis, T.; Studer, V.; Chen, Y.; Rossier, J.; Potier, M.C. Integrating whole transcriptome assays on a lab-on-a-chip for single cell gene profiling. Lab Chip 2008, 8, 443-450.

90. Marcus, J.S.; Anderson, W.F.; Quake, S.R. Microfluidic single-cell mRNA isolation and analysis. Anal. Chem. 2006, 78, 3084-3089.

91. Schell, W.A.; Benton, J.L.; Smith, P.B.; Poore, M.; Rouse, J.L.; Boles, D.J.; Johnson, M.D.; Alexander, B.D.; Pamula, V.K.; Eckhardt, A.E.; et al. Evaluation of a digital microfluidic real-time PCR platform to detect DNA of Candida albicans in blood. Eur. J. Clin. Microbiol. Infect. Dis. 2012, 31, 2237-2245.

92. Hua, Z.; Rouse, J.L.; Eckhardt, A.E.; Srinivasan, V.; Pamula, V.K.; Schell, W.A.; Benton, J.L.; Mitchell, T.G.; Pollack, M.G. Multiplexed real-time polymerase chain reaction on a digital microfluidic platform. Anal. Chem. 2010, 82, 2310-2316.

93. Xue, Z.; Huang, K.; Cai, C.; Cai, L.; Jiang, C.Y.; Feng, Y.; Liu, Z.; Zeng, Q.; Cheng, L.; Sun, Y.E.; et al. Genetic programs in human and mouse early embryos revealed by single-cell RNA sequencing. Nature 2013, 500, 593-597.

94. Niakan, K.K.; Han, J.; Pedersen, R.A.; Simon, C.; Pera, R.A. Human pre-implantation embryo development. Development 2012, 139, 829-841.

95. Zeng, F.; Baldwin, D.A.; Schultz, R.M. Transcript profiling during preimplantation mouse development. Dev. Biol. 2004, 272, 483-496.

96. Swain, J.E.; Smith, G.D. Advances in embryo culture platforms: Novel approaches to improve preimplantation embryo development through modifications of the microenvironment. Hum. Reprod. Update 2011, 17, 541-557.

97. Smith, G.D.; Swain, J.E.; Bormann, C.L. Microfluidics for gametes, embryos, and embryonic stem cells. Semin. Reprod. Med. 2011, 29, 5-14. 
98. Huang, H.Y.; Shen, H.H.; Tien, C.H.; Li, C.J.; Fan, S.K.; Liu, C.H.; Hsu, W.S.; Yao, D.J. Digital microfluidic dynamic culture of mammalian embryos on an electrowetting on dielectric (EWOD) chip. PLoS ONE 2015, 10, e0124196.

99. Egozcue, J.; Santalo, J.; Gimenez, C.; Durban, M.; Benet, J.; Navarro, J.; Vidal, F. Preimplantation genetic screening and human implantation. J. Reprod. Immunol. 2002, 55, 65-72.

100. Yan, L.; Wei, Y.; Huang, J.; Zhu, X.; Shi, X.; Xia, X.; Yan, J.; Lu, C.; Lian, Y.; Li, R.; et al. Advances in preimplantation genetic diagnosis/screening. Sci. China Life Sci. 2014, 57, $665-671$.

101. Harper, J.C.; Harton, G. The use of arrays in preimplantation genetic diagnosis and screening. Fertil. Steril. 2010, 94, 1173-1177. 


\section{Numerical Analysis of Hydrodynamic Flow in Microfluidic Biochip for Single-Cell Trapping Application}

\section{Amelia Ahmad Khalili and Mohd Ridzuan Ahmad}

Abstract: Single-cell analysis has become the interest of a wide range of biological and biomedical engineering research. It could provide precise information on individual cells, leading to important knowledge regarding human diseases. To perform single-cell analysis, it is crucial to isolate the individual cells before further manipulation is carried out. Recently, microfluidic biochips have been widely used for cell trapping and single cell analysis, such as mechanical and electrical detection. This work focuses on developing a finite element simulation model of single-cell trapping system for any types of cells or particles based on the hydrodynamic flow resistance (Rh) manipulations in the main channel and trap channel to achieve successful trapping. Analysis is carried out using finite element ABAQUS-FEA ${ }^{\mathrm{TM}}$ software. A guideline to design and optimize single-cell trapping model is proposed and the example of a thorough optimization analysis is carried out using a yeast cell model. The results show the finite element model is able to trap a single cell inside the fluidic environment. Fluid's velocity profile and streamline plots for successful and unsuccessful single yeast cell trapping are presented according to the hydrodynamic concept. The single-cell trapping model can be a significant important guideline in designing a new chip for biomedical applications.

Reprinted from Int. J. Mol. Sci. Cite as: Khalili, A.A.; Ahmad, M.R. Numerical Analysis of Hydrodynamic Flow in Microfluidic Biochip for Single-Cell Trapping Application. Int. J. Mol. Sci. 2015, 16, 26770-26785.

\section{Introduction}

Lab on a Chip (LOC) and Micro Total Analysis Systems ( $\mu$ TAS) have attracted researchers' attention in the areas of biotechnology and biomedical engineering. The rise in interest is due to the utilization of these devices in a broad range of biological and biomedical application areas including genomics, enzymatic analysis, disease diagnosis, cell treatment, drug screening, single-cell analysis, and drug delivery. In cellular biology, single-cell analysis refers to the study of individual cells isolated from tissues in multi-cellular organisms. Conventionally, cell analyses are conducted with large populations of cells and data measurement can only represent the average values summed over the responses of many cells. Therefore, single-cell analysis is important to obtain more precise information and to reveal the properties of individual cells and cell-to-cell differences [1].

In order to perform single-cell analysis in microfluidic devices, trapping of a single cell is necessary. A variety of techniques have been employed to trap an individual cell. For example, microwell-based [2-6], dielectrophoresis-based [7-11], and hydrodynamic-based [12-24] microfluidic devices for single-cell trapping have been developed in response to an increasing demand for simple yet reliable tools for high-throughput cell manipulation at the single cell level. 
In microwell-based platforms, a precise geometry design is required to achieve a high trapping efficiency [4]. Dielectrophoresis-based cell trapping applied a non-uniform AC field to manipulate polarized particles in suspension and is an effective technique to efficiently manipulate a single cell. However, it appears to damage the trapped cells, thus affecting the cell proliferation. Hydrodynamic trapping uses the altered fluidic resistance created by microstructures on a fluid path, such as sieve-like traps [23-25] or small trapping sites [12-17,26,27], to control the movement of cells in a microchannel. For straight or serpentine-shaped channels with trapping sites, the fluidic resistances of these channels are carefully calculated so that the fluid and cells in the main channel will preferentially flow into the trapping sites when they are empty, but bypass them when they are occupied with a cell. The main challenge in hydrodynamic trapping is that it requires a precise microfluidic control of multiple streams. Further investigation and optimization of cells' trapping efficiencies are still required [20].

The concept of hydrodynamic trapping for small trapping sites was originally proposed by Tan and Takeuchi [26]. However, a proof of concept is performed by experimental work only and no prior optimization of the microfluidic design through simulation works has been reported. From our point of view, this could probably involve high fabrication costs and it might be time consuming to find the optimized geometry through devices fabricated by trial and error. Therefore, our work is focused on developing the single-cell trapping model to produce a finite element simulation system that could be used to optimize a channel's geometry for any type of cells or particles. The model is developed based on hydrodynamic flow resistance $(R h)$ manipulation in the main channel and trap channel to achieve successful trapping. This study provides a proof of concept demonstration for a cell positioning platform to trap single cells and a guideline for designing and optimizing single-cell trapping channel is proposed. The example of a thorough optimization study is presented using a 5- $\mu \mathrm{m}$ yeast cell model. Microchannels' geometrical size optimization is carried out by manipulating the geometry of the trap channel, trap hole, and main channel. Numerical simulations are conducted to evaluate the cells' trapping efficiencies for a variety of geometrical parameters. Fluid's velocity profile and streamline plots are studied to explain the fluid's stream direction according to the hydrodynamic principles. The single-cell trapping system is dependent on the cell's size, as different cells require different optimized trapping channel sizes, trap hole's sizes, and main channel lengths (LMain). Therefore, it is important for us to optimize the channel's geometry before fabricating the real device to reduce time and fabrication costs.

\section{Results and Discussion}

\subsection{Verification of Hydrodynamic Trapping Concept}

The purpose of this finite element analysis is to verify the hydrodynamic trapping concept in the proposed model and to perform geometry optimization for efficient single-cell trapping. According to the hydrodynamic trapping concept proposed by Tan and Takeuchi [26], single-cell/particle trapping is achievable when the flow rate of trap channel to main channel $\left(Q_{\text {Trap }} / Q_{\text {Main }}\right)$ ratio is above 1 . To verify the concept, the cell trapping model with trap hole's width $\left(W_{\text {Hole }}\right) 2.0 \mu \mathrm{m}$ is used to study the appropriate flow resistance of main channel to trap channel ( $\left.R h_{\text {Main }} / R h_{\text {Trap }}\right)$ ratio. 
Main channel's length $\left(L_{\text {Main }}\right)$ is manipulated to create an $R h_{\text {Main }} / R h_{\text {Trap }}$ ratio ranging from 1 to 6 . Increasing the $R h_{\text {Main }} / R h_{\text {Trap }}$ ratio is proportional with the increase in the main channel's (loop path) length. A yeast cell model is successfully trapped when a $R h_{\text {Main }} / R h_{\text {Trap }}$ ratio of 3.5 or higher is used (Figure 1C,D). Furthermore, results show that an $R h_{\text {Main }} / R h_{\text {Trap }}$ ratio ranging between 1.0 and 3.0 caused the cell to bypass the trap channel (Figure 1A,B).

From the simulation result, an $R h_{\text {Main }} / R h_{\text {Trap }}$ ratio of 3.5 or above is found to be able to trap single cells via the hydrodynamic trapping concept. To further verify the principle of the hydrodynamic trapping, the fluid's velocity inside the main channel and trap channel before and after trapping is analyzed. The velocity of the fluid at two points is analyzed (Figure 2A) to represent the fluid's velocity before and after trapping for a cell trapping model with an $R h_{\text {Main }} / R h_{\text {Trap }}$ ratio of 3.5 or 4.5 (Figure $2 \mathrm{~B}$ ). The fluid's velocity in the main channel before cell trapping is found to be lower than the velocity in the trap channel (Figure 2B). However, after the cell is trapped inside the trap channel, the fluid's velocity inside the trap channel decreases instantly and the fluid's velocity at the main channel increases dramatically. This finding supports the principle of hydrodynamic trapping in which when the trapping side is empty, the trap channel will have lower flow resistance compared to the bypass channel (main channel). When the velocity of fluid in the trap channel is higher, it leads to a lower hydrodynamic resistance in the trapping site, which creates a trapping stream that will direct cells into the trap channel. When a cell has been trapped inside the trap channel, it blocks the trap hole and drastically decreases the fluid's velocity inside the trap channel. The direction of fluid flow diverges from the trap channel to the loop path (main channel). Therefore, subsequent cells will be directed to the loop path. The simulation results are found to be in good agreement with the reported experimental results. 


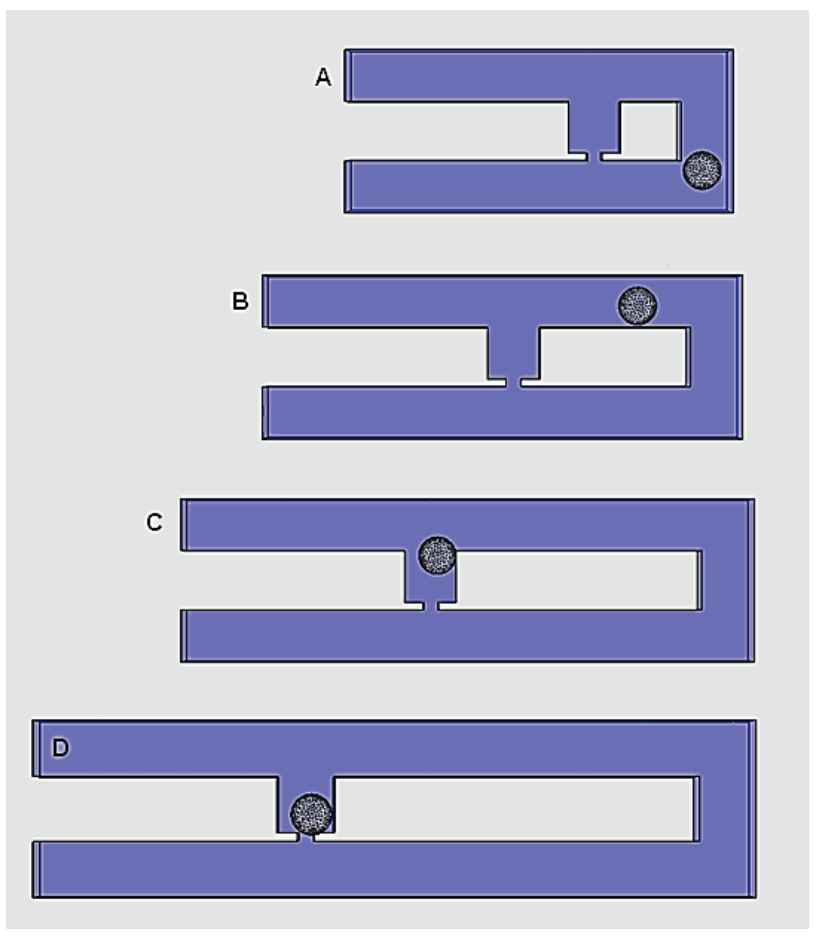

Figure 1. Cell trapping results at simulation time of $28 \mathrm{~s}$ for cell trapping model with $W_{\text {Hole }}$ of $2 \mu \mathrm{m}$ for different $R h_{\text {Main }} / R h_{\text {Trap }}$ ratio of (A) 1.5 ; (B) 2.5 ; (C) 3.5; and (D) 4.5.

\subsection{Effects of $R h_{\text {Main }} / R h_{\text {Trap }}$ Ratio in Cell Trapping}

Subsequent simulation is carried out to study the effects of the $R h_{\text {Main }} / R h_{\text {Trap }}$ ratio on cell trapping using a model with trap hole widths of 1.0 or $1.5 \mu \mathrm{m}$. The main channel's length has to be manipulated to comply with the desired $R h_{\text {Main }} / R h_{\text {Trap }}$ ratio. Similar trapping behavior is obtained when the $W_{\text {Hole }}$ is decreased to $1.5 \mu \mathrm{m}$. The hydrodynamic concept works accordingly and the yeast cell is able to be directed towards the trap channel by the fluid stream when the $R h_{\mathrm{Main}} / R h_{\mathrm{Trap}}$ ratio is 3.5 and above. However, for models with a trap holes width of $1.0 \mu \mathrm{m}$, a cell is not able to be trapped even though the $R h_{\text {Main }} / R h_{\text {Trap }}$ ratio is above 3.5. The cell is found to not be moving to the trap channel and bypasses it (data not shown). This result shows that a $W_{\text {Hole }}$ of $1 \mu \mathrm{m}$ is not suitable for the specified trap channel dimension $(7 \mu \mathrm{m}$ width, height, and length). The design fails to follow the hydrodynamic trapping concept, probably due to the small trap hole $(<1 / 5$ of trap channel's width $\left.\left(W_{\text {Trap }}\right)\right)$. The small $W_{\text {Hole }}$ probably cause a very low fluid velocity distribution and produce low pressure drop that unable to capture cells into the trap channel. [28]. A simulation study performed by Khalili et al. [28] showed the same trend of results when a very small $W_{\text {Hole }} / W_{\text {Trap }}$ is used $(<1 / 5)$. For designing a single-cell trapping channel, we suggest for the $W_{\text {Hole }}$ to

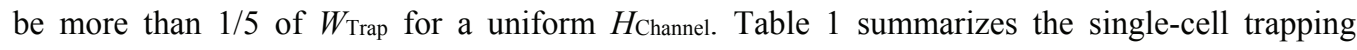

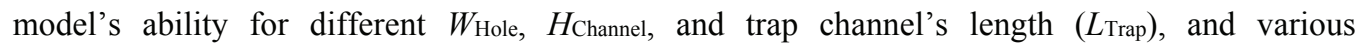
$R h_{\text {Main }} / R h_{\text {Trap }}$ ratios. 
A

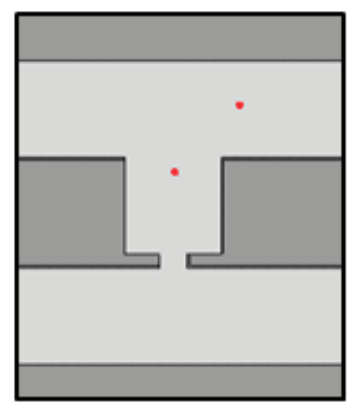

B

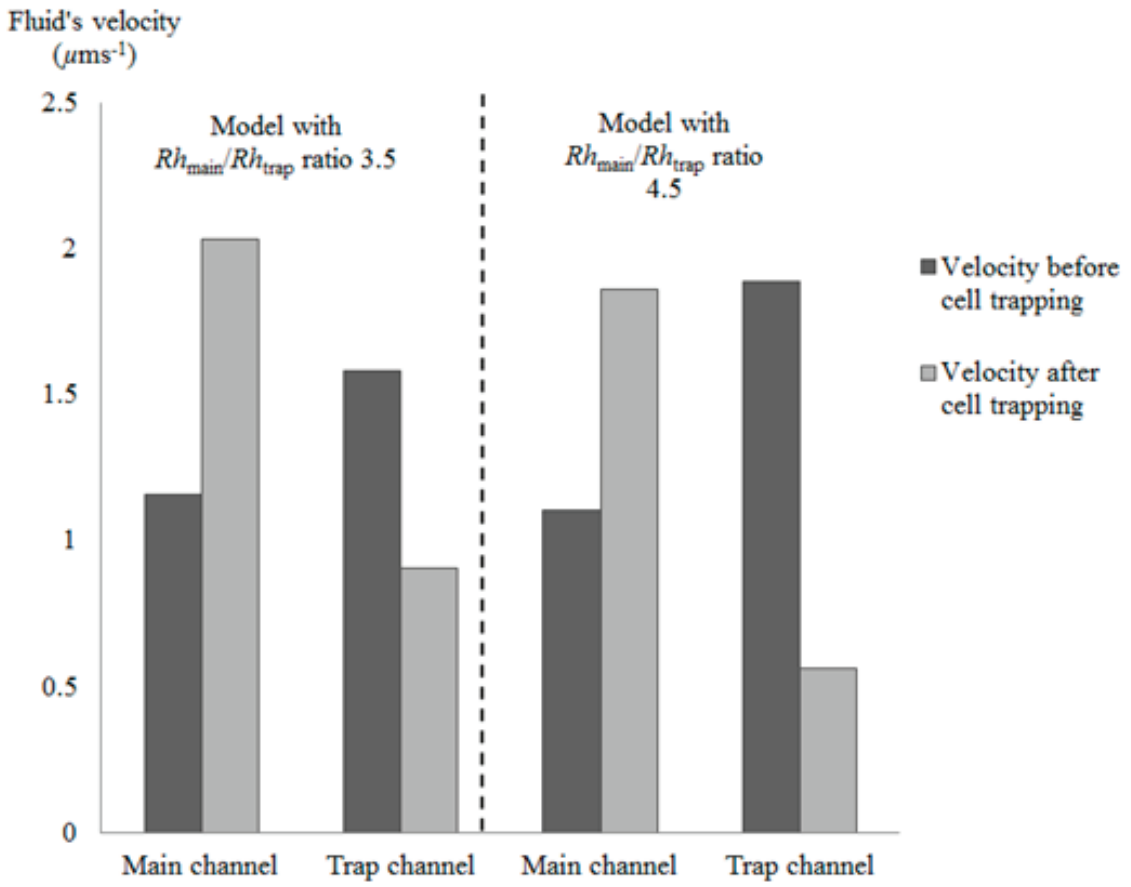

Figure 2. (A) Points representing velocity of fluid in the trap channel (left side) and main channel (right side); (B) graph representing velocity of fluid in trap channel and main channel for cell trapping model with $R h_{\text {Main }} / R h_{\text {Trap }}$ ratio of 3.5 or 4.5 before and after cell trapping. 


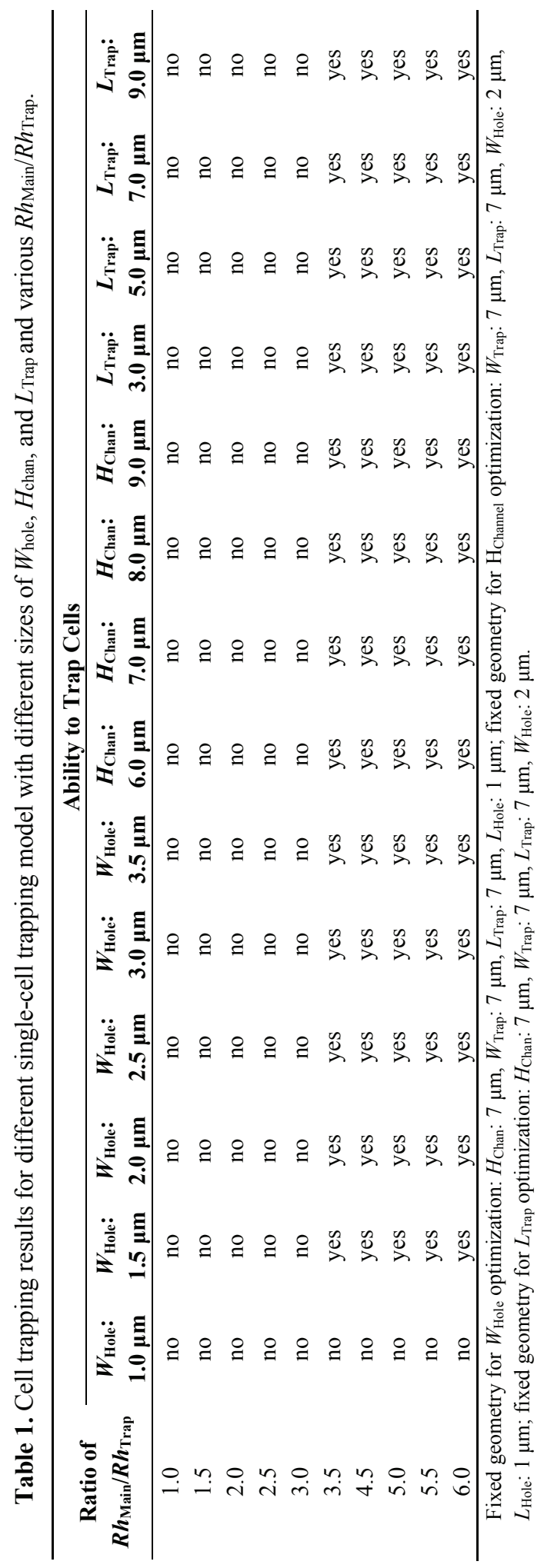


The fluid velocity profile and velocity streamline field of the cell trapping model are analyzed to understand the hydrodynamic trapping mechanism. Fluid velocity streamlines present the direction the fluid streams are heading, while velocity profiles represent the velocity value in the channel by the contour color. The velocity streamlines produced by the cell trapping model with an $R h_{\text {Main }} / R h_{\text {Trap }}$ ratio below 3.5 (Figure $3 \mathrm{~A}, \mathrm{~B}$ ), are found to be not fully directed to the trap channel and the portions of the streamlines that passed through the trap channel are directed to the loop. The produced fluid streams unable to direct the cell into the trap channel. This finding is in agreement with the fluid's velocity distribution produced by the same model (Figure 4A,B). Results show that the main channel's (loop path) fluid velocity for the single-cell trapping model with an $R h_{\text {Main }} / R h_{\text {Trap }}$ ratio of 1.5 and 2.5 is higher compared to the trap channel's fluid velocity. Therefore the main stream will direct the yeast cell to flow into the main channel's path and bypass the trap channel.

In contrast with the cell trapping model with an $\mathrm{RhMain} / \mathrm{Rh}_{\mathrm{Trap}}$ ratio of 3.5 and above (Figure 3C,D), the streamlines profiles show the fluid flow diverging from the main channel to the trap channel and directed towards the trap channel. For models with an $\mathrm{Rh}$ Main $/ \mathrm{Rh}_{\text {Trap }}$ ratio of 3.5 or 4.5 (Figure 4C,D), the fluid's velocity distribution from the trap hole to the trap channel is higher compared to the fluid's velocity in the main channel. These results show that the trap channel produces lower hydrodynamic resistance than the main channel and the mainstream will direct the yeast cell into the trap channel. Both models with an $\mathrm{Rh}$ Main $/ \mathrm{Rh}_{\text {Trap }}$ ratio of 3.5 and 4.5 produce almost similar fluid velocity patterns that will produce appropriate pressure drop for the cell to be trapped.

The hydrodynamic trapping concept is found to be ineffective for a cell trapping model with a $W_{\text {Hole }}$ of $1.0 \mu \mathrm{m}$. Subsequently, the $L_{\text {Main }}$ has been increased to obtain an $R h_{\text {Main }} / R h_{\text {Trap }}$ ratio between 3.5 and 6.0; however, the cell trapping is not successful. The fluid velocity streamlines produced by this model show different profiles compared to the streamlines produced by models with $W_{\text {Hole }}$ of $1.5 \mu \mathrm{m}$ and $2.0 \mu \mathrm{m}$ (Figure 5). The streamlines profile for the model shows that the flow direction is not fully focused into the trap channel but diverted to both the trap channel and the loop path directions (Figure 5A). The behavior of the fluid before cell bypass trap channel represents same trend of velocity profile and streamlines as models with unsuccessful trapping (Figure 3A,B). From the simulation results, the minimum main channel length needed to perform successful trapping is the length which produces an $R h_{\text {Main }} / R h_{\text {Trap }}$ ratio of 3.5 (with the exception of the model with $W_{\text {Hole }}$ of $\left.1.0 \mu \mathrm{m}\right)$.

Both cell trapping models with a trap hole width of $1.5 \mu \mathrm{m}$ or $2.0 \mu \mathrm{m}$ are found to be able to trap the yeast cell model with almost similar velocity profile. However, there are variations in the complete cell trapping time (time when the cell touches the surface of the trap channel) between different $R h_{\text {Main }} / R h_{\text {Trap }}$ ratios. A higher ratio requires a shorter time for the trapping process compared to a lower ratio. The graph in Figure 6 shows the results of trapping time for cell trapping models with $W_{\text {hole }}$ of 1.5 and $2.0 \mu \mathrm{m}$ for $R h_{\text {Main }} / R h_{\text {Trap }}$ ranging from 3.5 to 6.0 . From the graph, it is evident that the trapping time decreases with increasing $R h_{\text {Main }} / R h_{\text {Trap. This is probably }}$ due to the higher $R h_{\text {Main }} / R h_{\text {Trap }}$ ratio being able to perform velocity distribution in a shorter time compared to the lower $R h_{\text {Main }} / R h_{\text {Trap. A }}$ A greater $R h_{\text {Main }} / R h_{\text {Trap }}$ ratio could provide a lower hydrodynamic resistance in the trap channel and could transfer the fluid at a faster rate. The 
velocity distribution produces different pressure from the main channel to the trap hole, making the flow resistance inside the trap channel lower than the main channel. Therefore, together with the fluid, cells will flow to the lower flow resistance area and be trapped. A bigger $W$ hole value is able to produce shorter trapping time compared to the smaller height. Analyses are conducted for $W_{\text {hole }}$ of $2.5,3.0$, and $3.5 \mu \mathrm{m}$ and similar results are obtained where the cell is able to be trapped with $R h_{\text {Main }} / R h_{\text {Trap }}$ ratio of 3.5 and above (refer Table 1).

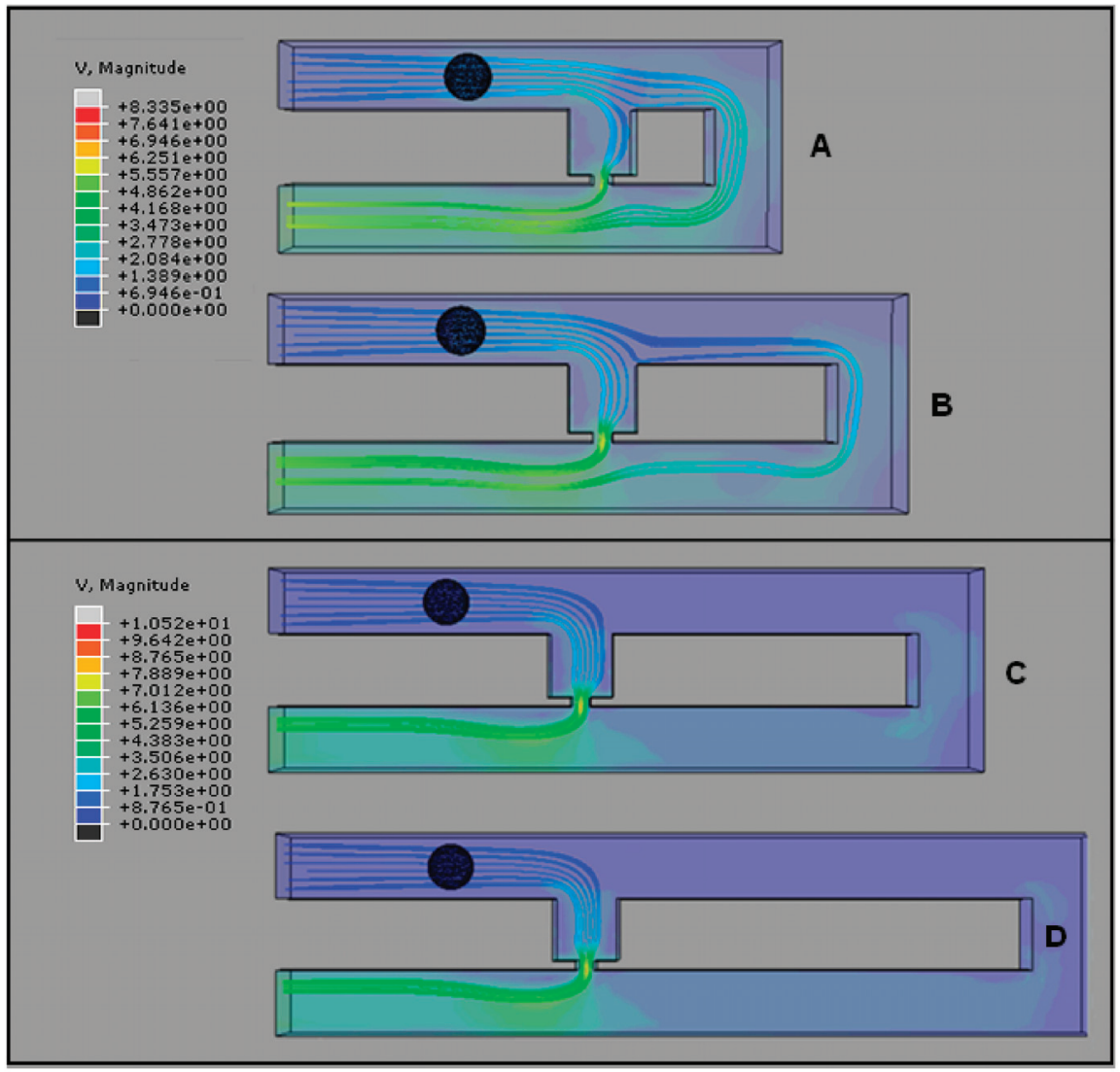

Figure 3. Velocity streamlines before cell trapping (top view) for cell trapping model with $W_{\text {Hole }}$ of $2 \mu \mathrm{m}$ for different $R h_{\text {Main }} / R h_{\text {Trap }}$ ratios of (A) 1.5 ; (B) 2.5 ; (C) 3.5; and (D) 4.5. V represents the fluid's velocity in $\mu \mathrm{ms}^{-1}$. 


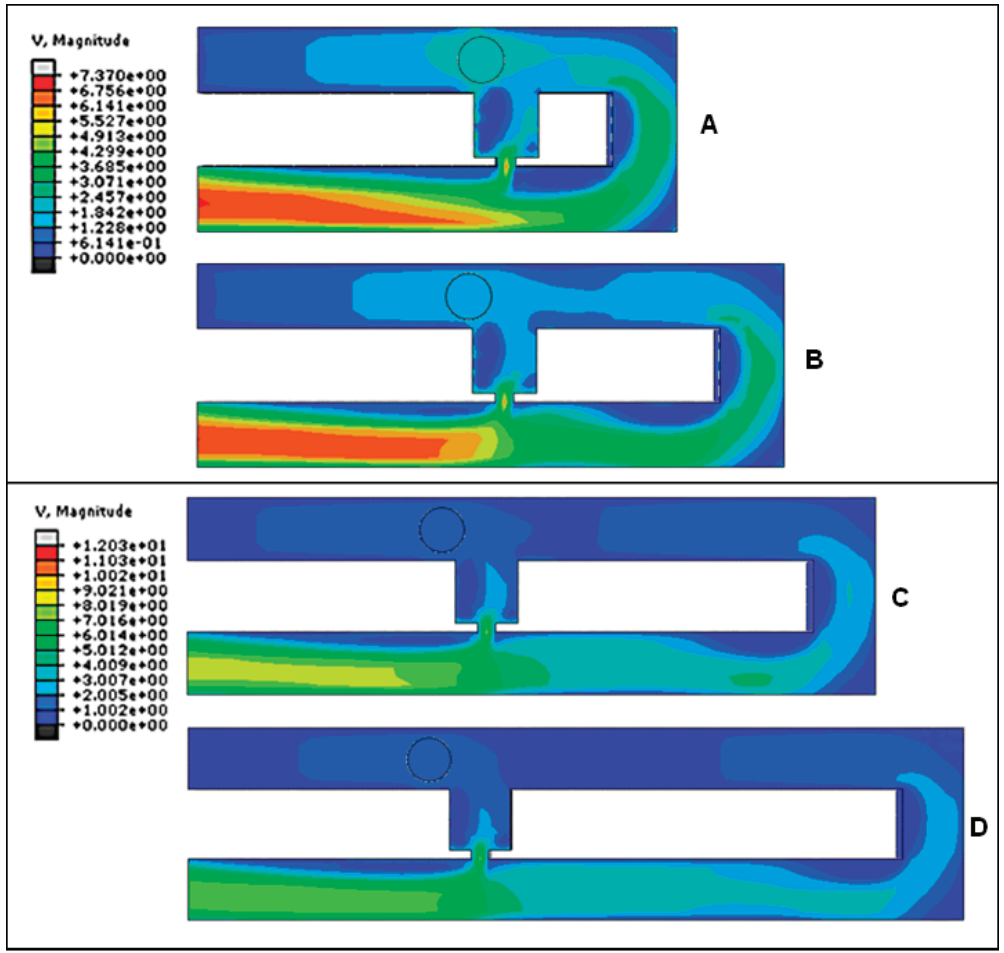

Figure 4. Velocity of fluid before cell trapping for single-cell trapping model with trapping hole width of $2 \mu \mathrm{m}$ for $R h_{\text {Main }} / R h_{\text {Trap }}$ ratios of (A) 1.5 ; (B) 2.5 ; (C) 3.5 ; and (D) 4.5. V represents the fluid's velocity in $\mu \mathrm{ms}^{-1}$.

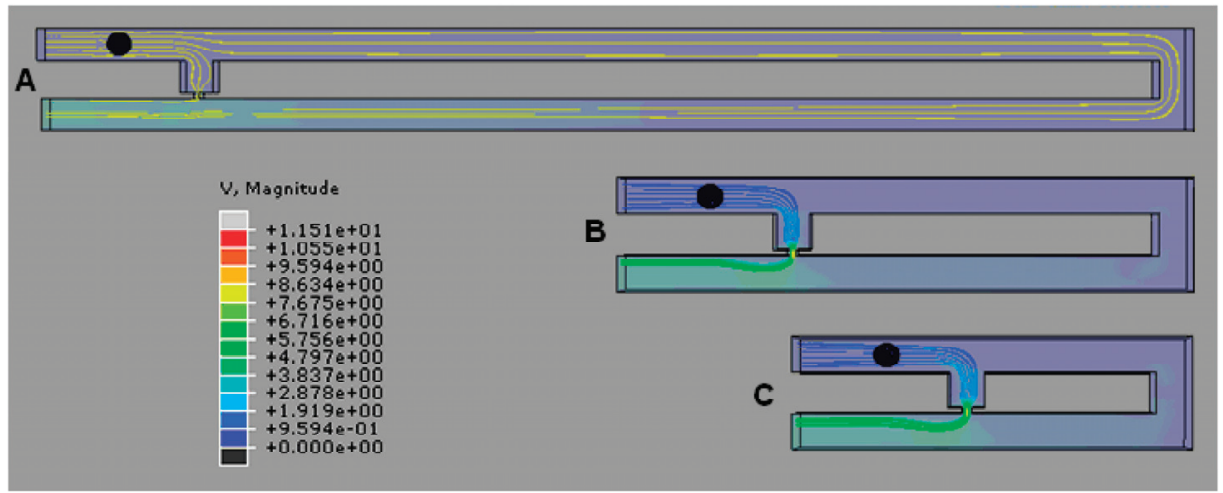

Figure 5. Velocity streamlines for the cell trapping model (top view) with $R h_{\text {Main }} / R h_{\text {Trap }}$ ratio of 3.5 for model with trap hole width of (A) $1.0 \mu \mathrm{m}$; (B) $1.5 \mu \mathrm{m}$; or (C) $2.0 \mu \mathrm{m}$. V represents the fluid's velocity in $\mu \mathrm{ms}^{-1}$. 


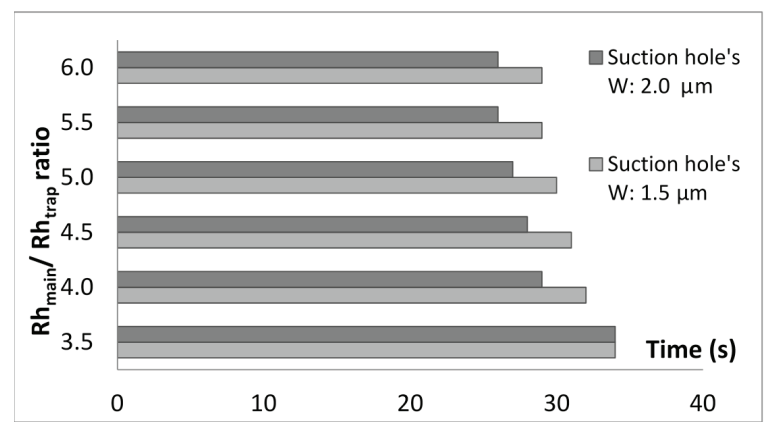

Figure 6. Cell trapping time for model with different $R h_{\text {Main }} / R h_{\text {Trap }}$ ranging from 3.5 to 6.0 for single-cell trapping model with trapping hole widths of 1.5 and $2.0 \mu \mathrm{m}$.

\subsection{Optimization of Trap Channel's Length}

After investigating the effects of $R h_{\text {Main }} / R h_{\text {Trap }}$ ratio for the single cell trapping model, the efficiency of the single-cell trapping is enhanced by optimizing the trap channel's length $\left(L_{\mathrm{Trap}}\right)$ (refer Figure 2A). Using yeast cell and four different $L_{\text {Trap }}$, the behavior of cell trapping is observed. A model with $L_{\text {Trap }}$ of $3 \mu \mathrm{m}$ is able to trap single cells; however, after a cell is trapped, both of the paths to the loop and the outlet will eventually be blocked, causing clogging of subsequent cells at the main channel and thus preventing the smooth movement of cells towards the outlet (Figure 7A). Therefore the length is not suitable for efficient cell trapping. From the results, a model with $L$ Trap of $5 \mu \mathrm{m}$ is found to be the most suitable length to trap a 5 - $\mu \mathrm{m}$ yeast cell as it could allow the subsequent cell to flow to the loop and heading to the outlets (Figure 7B). For a model with $L$ Trap of 7 or $9 \mu \mathrm{m}$, results show that two cells are able to enter the trap channel during cell trapping (Figure 7C,D). The aim for the cell trapping model development is to trap a single cell; therefore the $L_{\text {Trap }}$ of 7 and $9 \mu \mathrm{m}$ are not suitable for efficient single-cell trapping.

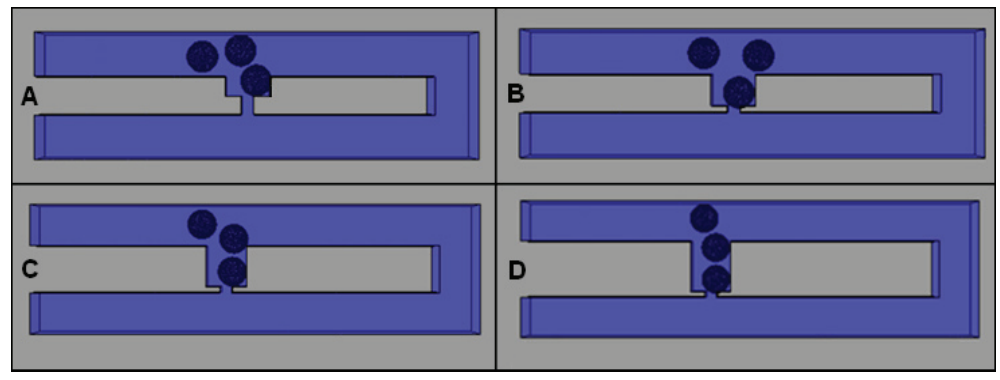

Figure 7. Cell trapping results for the optimization of different $L_{\text {Trap }}$ values: (A) $3 \mu \mathrm{m}$; (B) $5 \mu \mathrm{m}$; (C) $7 \mu \mathrm{m}$; and (D) $9 \mu \mathrm{m}$. 


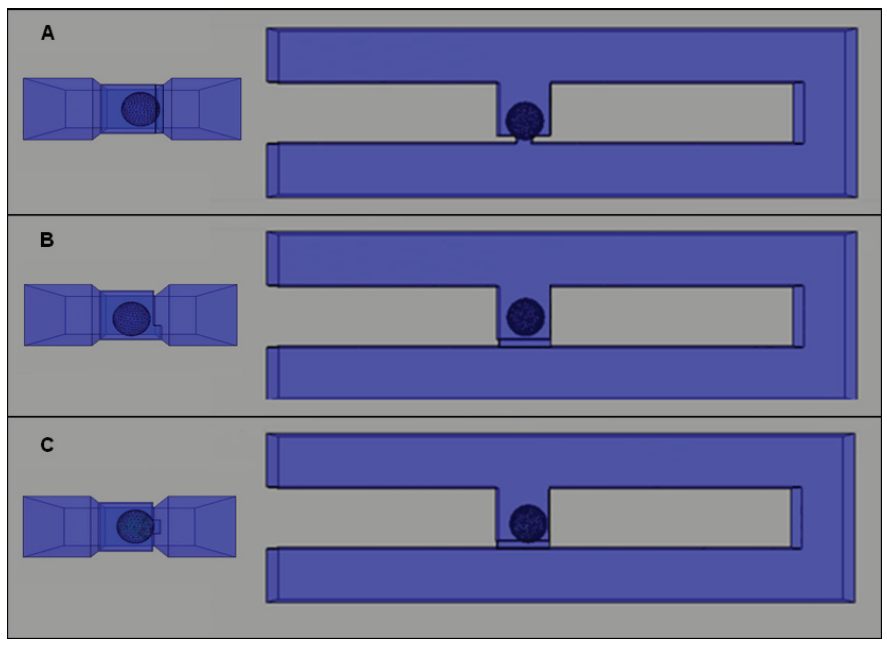

Figure 8. Cell trapping results at simulation time of $34 \mathrm{~s}$ for cell trapping model with the same trap hole size (2, 7 and $7 \mu \mathrm{m}$ of $W, L$ and $H$, respectively) for an $R h_{\text {Main }} / R h_{\text {Trap }}$ ratio of 3.5 at three different positions: $(\mathbf{A})$ model A; (B) model B; and $(\mathbf{C})$ model C. (Left) side view; (right) top view of the model.

\subsection{Effects of Different Trap Hole Positions}

The final analysis is carried out to study the effects of the trap hole's position on the cell trapping. Analysis is carried out using a cell trapping model with an $R h_{\text {Main }} / R h_{\text {Trap }}$ ratio of 3.5 and trap hole dimensions of 1,7 , and $2 \mu \mathrm{m}$ in length, height, and width, respectively. Three different trap hole positions with similar dimensions are analyzed as illustrated in Figure 12, namely models A-C. Cell trapping results demonstrate that all of the models are able to trap cells with an $R h_{\text {Main }} / R h_{\text {Trap }}$ ratio of 3.5 (Figure 8 ). The streamlines velocity fields produced by the models before cell trapping are focused towards the trap channel. The streamlines show that the fluid stream produced is fully directed toward the trap channel with a similar pattern. The only difference in the streamlines pattern between the models is the position of streams towards the trap hole (Figure 9B,D). The differences could be observed by viewing the streamlines at three different views (Figure 9A). For model A, the streamlines' focusing could be clearly seen from the top view, where the streamlines' direction focused on the center of the trap channel (Figure 9A(i)). For models B and $\mathrm{C}$, the streamline focusing could be clearly differentiated by viewing from the front and side (Figure 9C,D(i,ii)), where model B streamlines are focused at the base of the channel while model $\mathrm{C}$ are focused at the middle of the trap channel. The findings suggest that a cell trapping model with similar $R h_{\text {Main }} / R h$ Trap ratio produces similar trapping behavior despite the different trap hole's positions. 
A
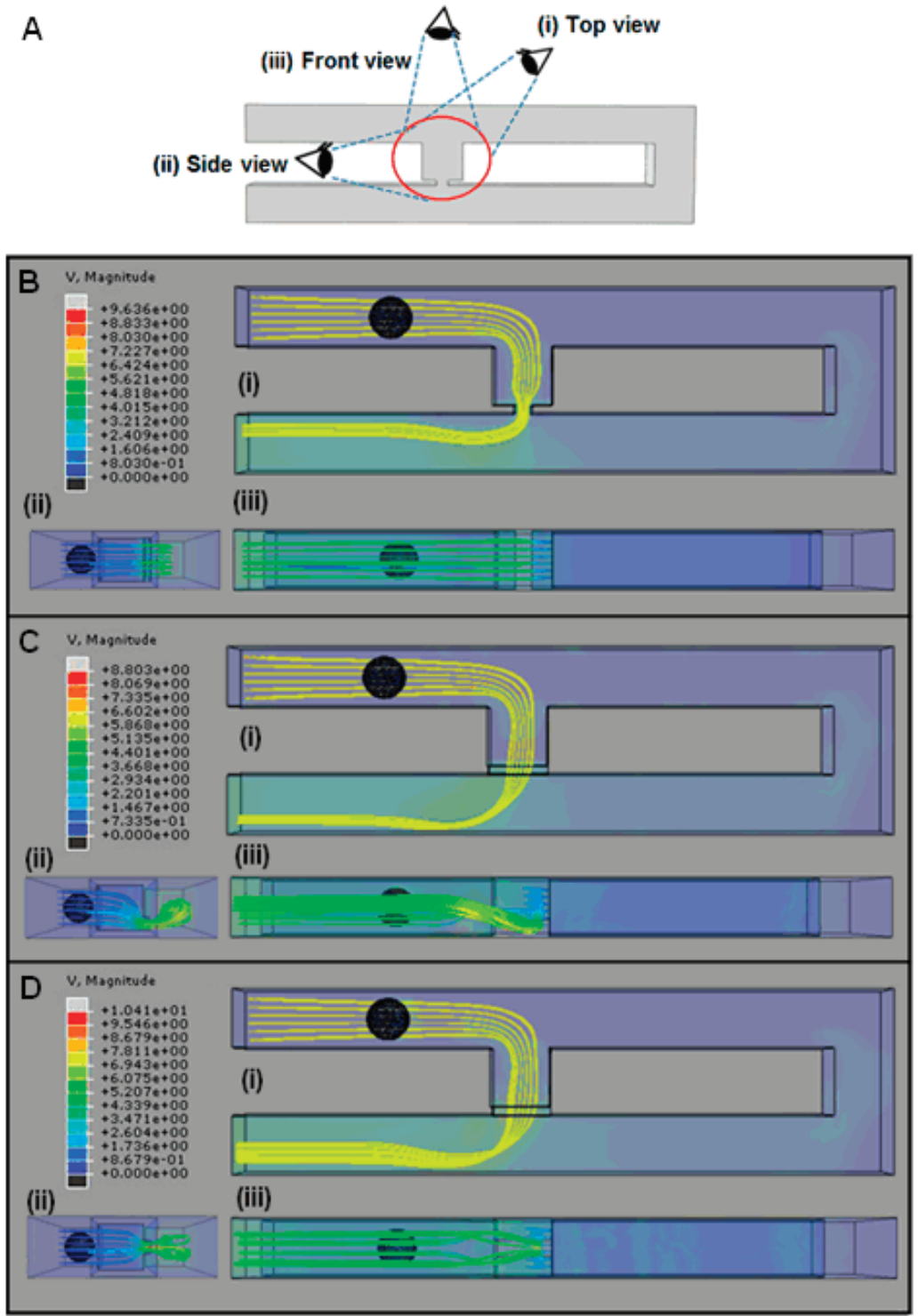

Figure 9. Streamline velocity for three different cell trapping models with the same trap hole size ( $2 \mu \mathrm{m}, 7 \mu \mathrm{m}$, and $7 \mu \mathrm{m}$ of $W, L$, and $H$, respectively) for an $R h_{\text {Main }} / R h_{\text {Trap }}$ ratio of 3.5 at three different positions: (A) model A; (B) model B; and (C) model C. (Left) side view; (right) top view (top) and front view (bottom) of the model. V represents the fluid's velocity in $\mu \mathrm{ms}^{-1}$.

\section{The Concept of the Model}

The hydrodynamic trapping concept can be summarized as follows: (a) the trapping channel has a lower $R h$ than the by-passing channel when a trapping site is empty, and will make the 
particles/cells flow into the trapping stream and directed into the trap; (b) when a bead/cell is trapped, it will act as a plug and will increase the $R h$ along the trap channel drastically; and (c) the main flow will change from the trap channel to the by-pass channel (main channel) and the next particles/cells will be directed to the by-pass stream, passing by the filled trapping site [29]. Figure 10 shows a schematic explanation of the hydrodynamic trapping concept with $R h_{\text {Trap }}$ and $R h_{\text {Main }}$ representing the flow resistance of trap channel and main channel, respectively. The yellow circle denotes a yeast cell that needs to be trapped.

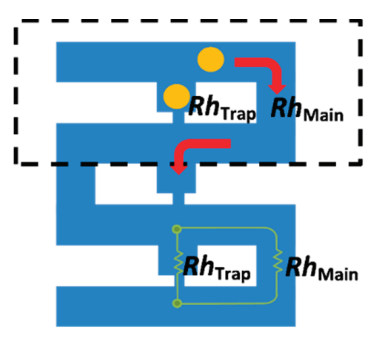

Figure 10. Simple schematic of single-cell trapping channel with the hydrodynamic resistance.

The Darcy-Weisbach equation is used to determine the pressure drop or pressure difference in a microchannel and solve the continuity and momentum equations for the Hagen-Poiseuille flow problem. From the Hagen-Poiseuille equation, the flow rate $(Q)$ can be defined by the following equation:

$$
\Delta P=Q \times R h=Q \times\left(\frac{C \mu L P^{2}}{A^{3}}\right)
$$

where $\Delta P$ is the pressure drop, $R h$ is the flow resistance of the rectangular channels, $C$ is a constant that depends on the aspect ratio (ratio between height and width of the channel), $\mu$ is the fluid's viscosity, and $L, P$, and $A$ are the length, perimeter, and cross-sectional area of the channel, respectively.

From Equation (1), by approximating that the pressure drop across the trap channel and the main channel are the same $\left(\Delta P_{\text {Trap }}=\Delta P_{\text {Main }}\right)$, the flow rate ratio $\left(Q_{\text {Trap }} / Q_{\text {main }}\right)$ or flow resistance ratio ( $R h_{\text {Main }} / R h_{\text {Trap }}$ ) between the trap channel and the main channel can be given as follows [30]:

$$
\frac{Q_{\text {Trap }}}{Q_{\text {Main }}}=\frac{R h_{\text {Main }}}{R h_{\text {Trap }}}=\left(\frac{C_{\text {Main }}}{C_{\text {Trap }}}\right)\left(\frac{L_{\text {Main }}}{L_{\text {Trap }}}\right)\left(\frac{P_{\text {Main }}}{P_{\text {Trap }}}\right)^{2}\left(\frac{A_{\text {Trap }}}{A_{\text {Main }}}\right)^{3}
$$

By using a relationship of $A=W \times H$ and $P=2(W+H)$, where $W$ and $H$ are the width and height of the channel, respectively, Equation (2) can be defined as:

$$
\frac{Q_{\text {Trap }}}{Q_{\text {Main }}}=\frac{R h_{\text {Main }}}{R h_{\text {Trap }}}=\left(\frac{C_{\text {Main }}}{C_{\text {Trap }}}\right)\left(\frac{L_{\text {Main }}}{L_{\text {Trap }}}\right)\left(\frac{W_{\text {Main }}+H_{\text {Main }}}{W_{\text {Trap }}+H_{\text {Trap }}}\right)^{2}\left(\frac{W_{\text {Trap }} H_{\text {Trap }}}{W_{\text {Main }} H_{\text {Main }}}\right)^{3}
$$

From Equations (2) and (3), it is noted that the flow rates of the trap channel ( $\left.Q_{\text {Trap }}\right)$ and the main channel ( $Q_{\text {Main }}$ ) are distributed depending on the corresponding $R h$. For the trap to work, the flow rate along the trap channel must be greater than that of main channel ( $\left.Q_{\text {Trap }}>Q_{\text {Main }}\right)$. In other 
words, the flow resistance along the main channel must be greater than that of the trap channel $\left(R h_{\text {Main }}>R h_{\text {Trap }}\right)$. Therefore, a single cell can be trapped by manipulating the flow resistance ratio ( $R h_{\text {Main }} / R h_{\text {Trap }}$ ), which is determined by the geometric parameters of the channels.

A single-cell trapping model is developed to produce a finite element single-cell trapping system in which the optimization of a channel's geometry, dependent on the desired cell size, could be performed. The geometry of the trapping channel is a variable for optimization (see Equation (3)) and subject to the size of cells and the application that will be carried out in the channel after the cells are trapped. An example of a thorough optimization study is presented in this paper using a $5-\mu \mathrm{m}$ yeast cell model. For other cell sizes, a guideline for designing and optimizing the cell trapping channel is proposed. Firstly the diameter of the viable cells in suspension (floating cells) before cell adhesion occur (for adherent type of cells) should be determined. This is important to determine the range of suitable trap hole sizes. We suggest that the $W_{\text {Hole }}$ to be less than one third of the cell's size due to the ability of cells to deform and the flexibility to enter the trap hole instead of being trapped in the trap channel [30]. This could happen, especially to cells that have no cell wall such as human cells. Next, after determining $W_{\text {Hole }}$, the $H_{\text {Channel }}$ and $W_{\text {Trap }}$ have to be optimized. $H_{\text {Channel }}$ should be bigger than the diameter of the cells to reduce friction between the cell surface and the channel's wall and to avoid cell squeezing (for applications that do not require cells to be squeezed, e.g., cell culturing, drugs treatment, and cell adhesion study). The optimization of the $L_{\text {Trap }}$, is dependent on the application of cells after being trapped. Long $L_{\text {Trap }}$ could cause more than one cell to be trapped if cells in suspension are very near to each other. However, for long-term monitoring of cell behavior for Tetrahymena thermophila, a long trap channel is needed to avoid cell from swimming back to the main channel [30]. The trap channel's geometry size choices are dependent on the application of the trapping platform after the cells/particles are trapped. For example, if adherence cells are used and need to be cultured inside the trapping platform, the $W_{\text {Trap }}$ should be bigger than the diameter of the cell (viable cells in suspension before adhesion). This is because cells need space for cell adhesion and spreading as the diameter of cells after adhesion will increase depending on culture time. In different applications, individual ciliate protozoan, Tetrahymena thermophila [30] need to be trapped and maintained in the trap channel for long-term monitoring of cell behavior. Therefore, no expansion in size is expected after the trapping process and the trap channel's width does not require space for expansion. In summary, the geometry of channels is a variable ( $L, H$, and $W$; see Equation (3)) for optimization, subject to the size of cells used and the application that will be carried out after the cells are trapped.

In this single-cell trapping model, cells are introduced into the device through the inlet with an appropriate flow rate and directed to the trap channel by optimizing the channel's geometry. Trap hole and trap channel geometry are optimized and $L$ Main is manipulated to produce an appropriate $R h$ ratio that leads to successful trapping (see Equation (3)). The excess and remaining cells will be directed out through the channel's outlet by injecting cell's culture medium. The appropriate channel's geometry to trap a 5- $\mu \mathrm{m}$ single yeast cell in the specified design is studied. The finite element single-cell trapping model is focusing only on a single trap channel (see dashed box in Figure 1) for geometry optimization due to the complexity and high processing time required for the analysis. 


\section{Simulation Setup}

The analysis is carried out using finite element ABAQUS-FEA ${ }^{\mathrm{TM}}$ analysis software, which can perform multiphysics analyses. The single-cell trapping model consists of two different parts, the Eulerian part as the fluid channel and a three-dimensional (3D) deformable part as the sphereshaped elastic yeast cell model (Figure 11A,B). The fluid consists of two microchannels, the main channel (loop channel) and a trap channel with a rectangular trap hole placed in the center, at the edge of the trap channel. The microchannel is modeled as 3D Eulerian explicit EC3DR and an eight-node linear Eulerian brick element part assigned with water properties (density, equation of state, and viscosity). A sphere-shaped yeast cell ( $5 \mu \mathrm{m}$ in diameter) is modeled as an elastic 3D standard solid deformable C3D8R and an eight-node linear brick 3D part with the yeast properties (Young's modulus, Poisson's ratio, and density) obtained from literature [31-38].

Figure 11C shows the assembly setup with a yeast cell positioned in the main channel, near the channel's inlet (left). The parts are assembled to develop the finite element model for the proposed system (Figure 11C). The initial position of the cell is fixed (same distance between cell and trap channel) for all models. Interaction between cell and water is set as general contact with rough tangential behavior and the interaction between cell surface and channel's wall is set as frictionless. The fluid channel and cell are meshed using hexahedron mesh types. Total mesh elements for the cell trapping model ranged from 10,627 to 22,485 elements. No-inflow and non-reflecting outflow Eulerian boundary conditions are applied to the channel's wall. A constant inflow velocity of $0.5 \mu \mathrm{ms}^{-1}$ is applied to the inlet and atmosphere pressure is applied to the outlet of the channel.

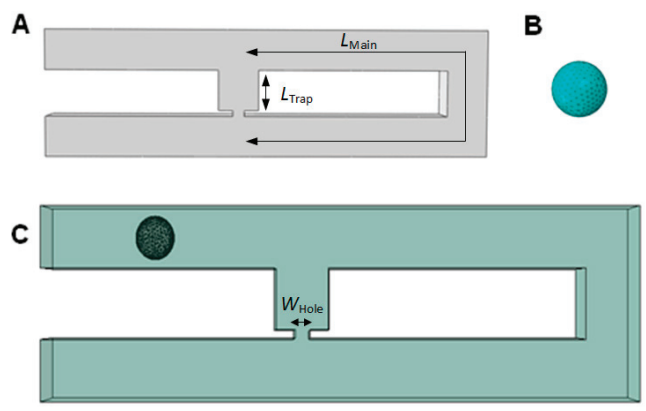

Figure 11. Construction of the finite element model of single-cell trapping system and parts involved: (A) Eulerian part (fluid channel's top view) $L_{\text {Main }}$ represents the main channel's length and $L$ Trap represents the trap channel's length; (B) 3D deformable part (yeast cell model); (C) simulation's assembly setup (cell is positioned between inlet and trap channel as initial position). $W_{\text {Hole }}$ represents trap hole's width.

The simulation analysis could be divided into four parts: the verification of the hydrodynamic trapping concept, the effects of $R h_{\text {Main }} / R h_{\text {Trap }}$ ratio in cell trapping, the optimization of the trap channel's length, and the effects of the trap hole's position. For the verification of the hydrodynamic trapping concept, a model with a trap hole's width of $2.0 \mu \mathrm{m}$ is used for the analysis. To study the effects of $R h_{\text {Main }} / R h_{\text {Trap }}$ ratio in cell trapping, various $L$ Main ranging from 46 to $268 \mu \mathrm{m}$ 
(Figure $11 \mathrm{~A}$ ) and $W_{\text {Hole }}$ ranging from 1.0 to $3.5 \mu \mathrm{m}$ (Figure $2 \mathrm{C}$ ) with fixed $L_{\text {Hole }}$ of $1 \mu \mathrm{m}$ are applied to obtain the appropriate $R h_{\text {Main }} / R h_{\text {Trap }}$ ratio for cell trapping. The height of main channel, trap channel, and trap hole are uniform ( $\left.H_{\text {Channel }}\right)$ and were tested in the range of 6-9 $\mu \mathrm{m}$ and set to be $7 \mu \mathrm{m}$ throughout the analysis. For trap channel length $\left(L_{\text {Trap }}\right)$ optimization, various trap channel lengths from 3 to $9 \mu \mathrm{m}$ and a fixed trap channel width of $7 \mu \mathrm{m}$ are used, with three yeast cells in the analysis. Lastly, to study the effects of trap hole's position, three different positions for similar trap hole's dimensions are studied to observe the ability of the model for cell trapping. Figure 12 shows the different views for the three different positions, represented by models A, B, and C.
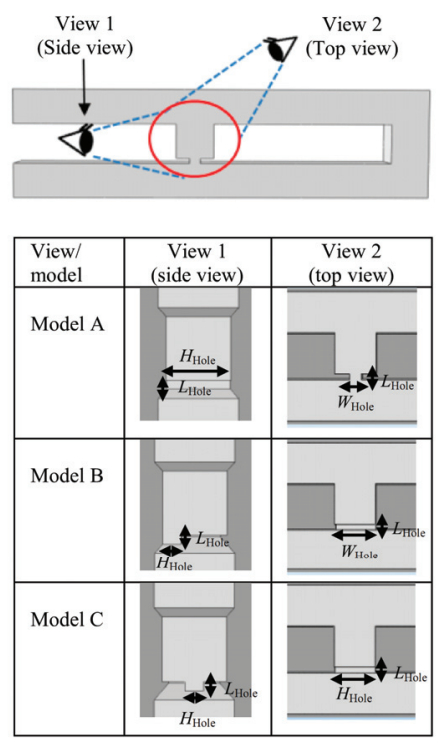

Figure 12. The views for three cell trapping models with the same trap hole size (2, 7 and $7 \mu \mathrm{m}$ for $W_{\text {Hole }}, L_{\text {Hole, }}$ and $H_{\text {Hole }}$, respectively) at different positions.

\section{Conclusions}

This study presents the finite element model of single-cell trapping inside microfluidic channel. This single-cell trapping system is constructed using Abaqus-FEA ${ }^{\mathrm{TM}}$ software. A guideline to design and optimize single-cell trapping model is proposed and the example of a thorough optimization analysis is carried out using a yeast cell model. The results show that the finite element model is able to trap a single cell inside the fluidic environment. The fluid velocity profile and streamline plots of successful and unsuccessful single yeast cell trapping are presented according to the hydrodynamic concept. This cell trapping model is able to isolate an individual yeast cell inside a fluidic environment, thus providing a platform for further single-cell mechanical or biological study. Single-cell manipulation such as chemical and biophysical treatments and also mechanical characterization could be performed inside the microfluidic channel using this system. The single-cell trapping model can be a significant important guideline in designing a new chip for biomedical applications. 
Acknowledgments: The research was supported by the Ministry of Higher Education of Malaysia (grant Nos. 4L640 and 4F351), and Universiti Teknologi Malaysia (grant Nos. 02G46, 03H82, and 03H80); we thank them for funding this project and for their endless support. The authors would like to express their heartiest appreciation to Mohd Ariffanan Mohd Basri from the Faculty of Electrical Engineering, Universiti Teknologi Malaysia for his contribution of ideas and valuable discussion during the theoretical development of this study.

Author Contributions: Amelia Ahmad Khalili and Mohd Ridzuan Ahmad wrote and edited the article, respectively.

Conflicts of Interest: The authors declare no conflict of interest.

\section{References}

1. Johann, R.M. Cell Trapping in Microfluidic Chips. Anal. Bioanal. Chem. 2006, 385, 408-412.

2. Lee, G.-H.; Kim, S.-H.; Kang, A.; Takayama, S.; Lee, S.-H.; Park, J.Y. Deformable L-shaped microwell array for trapping pairs of heterogeneous cells. J. Micromech. Microeng. 2015, 25, doi:10.1088/0960-1317/25/3/035005.

3. Sun, T.; Kovac, J.; Voldman, J. Image-based single-cell sorting via dual-photopolymerized microwell arrays. Anal. Chem. 2014, 86, 977-981.

4. Rettig, J.R.; Folch, A. Large-scale single-cell trapping and imaging using microwell arrays. Anal. Chem. 2005, 77, 5628-5634.

5. Tang, J.; Peng, R.; Ding, J. The Regulation of Stem cell differentiation by cell-cell contact on micropatterned material surfaces. Biomaterials 2010, 31, 2470-2476.

6. Doh, J.; Kim, M.; Krummel, M.F. Cell-laden microwells for the study of multicellularity in lymphocyte fate decisions. Biomaterials 2010, 31, 3422-3428.

7. Chen, N.-C.; Chen, C.-H.; Chen, M.-K.; Jang, L.-S.; Wang, M.-H. Single-cell trapping and impedance measurement utilizing dielectrophoresis in a parallel-plate microfluidic device. Sens. Actuators B Chem. 2014, 190, 570-577.

8. Sen, M.; Ino, K.; Ramon-Azcon, J.; Shiku, H.; Matsue, T. Cell pairing using a dielectrophoresis-based device with interdigitated array electrodes. Lab Chip 2013, 13, 3650-3652.

9. Voldman, J.; Gray, M.L.; Toner, M.; Schmidt, M.A. A Microfabrication-based dynamic array cytometer. Anal. Chem. 2002, 74, 3984-3990.

10. Thomas, R.S.; Morgan, H.; Green, N.G. Negative DEP traps for single cell immobilisation. Lab Chip 2009, 9, 1534-1540.

11. Gray, D.S.; Tan, J.L.; Voldman, J.; Chen, C.S. Dielectrophoretic registration of living cells to a microelectrode array. Biosens. Bioelectron. 2004, 19, 771-780.

12. Chen, Y.-C.; Allen, S.G.; Ingram, P.N.; Buckanovich, R.; Merajver, S.D.; Yoon, E. Single-cell migration chip for chemotaxis-based microfluidic selection of heterogeneous cell populations. Sci. Rep. 2015, 5, 1-13. 
13. Jin, D.; Deng, B.; Li, J.X.; Cai, W.; Tu, L.; Chen, J.; Wu, Q.; Wang, W.H. A Microfluidic device enabling high-efficiency single cell trapping. Biomicrofluidics 2015, 9, doi:10.1063/1.4905428.

14. Benavente-Babace, A.; Gallego-Pérez, D.; Hansford, D.J.; Arana, S.; Pérez-Lorenzo, E.; Mujika, M. Single-cell trapping and selective treatment via co-flow within a microfluidic platform. Biosens. Bioelectron. 2014, 61, 298-305.

15. Kim, J.; Erath, J.; Rodriguez, A.; Yang, C. A High-efficiency microfluidic device for size-selective trapping and sorting. Lab Chip 2014, 14, 2480-2490.

16. Lee, P.J.; Hung, P.J.; Shaw, R.; Jan, L.; Lee, L.P. Microfluidic application-specific integrated device for monitoring direct cell-cell communication via gap junctions between individual cell pairs. Appl. Phys. Lett. 2005, 86, doi:10.1063/1.1938253.

17. Frimat, J.-P.; Becker, M.; Chiang, Y.-Y.; Marggraf, U.; Janasek, D.; Hengstler, J.G.; Franzke, J.; West, J. A microfluidic array with cellular valving for single cell co-culture. Lab Chip 2011, 11, 231-237.

18. Kim, H.; Lee, S.; Kim, J. Hydrodynamic trap-and-release of single particles using dual-function elastomeric valves: design, fabrication, and characterization. Microfluid. Nanofluid. 2012, 13, 835-844.

19. Arakawa, T.; Noguchi, M.; Sumitomo, K.; Yamaguchi, Y.; Shoji, S. High-throughput single-cell manipulation system for a large number of target cells. Biomicrofluidics 2011, 5, doi:10.1063/1.3567101.

20. Kobel, S.; Valero, A.; Latt, J.; Renaud, P.; Lutolf, M. Optimization of microfluidic single cell trapping for long-term on-chip culture. Lab Chip 2010, 10, 857-863.

21. Hong, S.; Pan, Q.; Lee, L.P. Single-cell level co-culture platform for intercellular communication. Integr. Biol. 2012, 4, 374-380.

22. Shi, W.; Qin, J.; Ye, N.; Lin, B. Droplet-based microfluidic system for individual caenorhabditis elegans assay. Lab Chip 2008, 8, 1432-1435.

23. Di Carlo, D.; Aghdam, N.; Lee, L.P. Single-cell enzyme concentrations, kinetics, and inhibition analysis using high-density hydrodynamic cell isolation arrays. Anal. Chem. 2006, 78, 4925-4930.

24. Skelley, A.M.; Kirak, O.; Suh, H.; Jaenisch, R.; Voldman, J. Microfluidic control of cell pairing and fusion. Nat. Methods 2009, 6, 147-152.

25. Di Carlo, D.; Wu, L.Y.; Lee, L.P. Dynamic single cell culture array. Lab Chip 2006, 6, $1445-1449$.

26. Tan, W.-H.; Takeuchi, S. A Trap-and-release Integrated microfluidic system for dynamic microarray applications. Proc. Natl. Acad. Sci. USA 2007, 104, 1146-1151.

27. Chung, K.; Rivet, C.A.; Kemp, M.L.; Lu, H.; States, U. Imaging single-cell signaling dynamics with a deterministic high-density single-cell trap array. Anal. Chem. 2011, 83, 7044-7052.

28. Khalili, A.A.; Basri, M.A.M.; Ahmad, M.R. Simulation of single cell trapping via hydrodynamic manipulation. J. Teknol. 2014, 69, 121-126. 
29. Teshima, T.; Ishihara, H.; Iwai, K.; Adachi, A.; Takeuchi, S. A dynamic microarray device for paired bead-based analysis. Lab Chip 2010, 10, 2443-2448.

30. Kumano, I.; Hosoda, K.; Suzuki, H.; Hirata, K.; Yomo, T. Hydrodynamic trapping of tetrahymena thermophila for the long-term monitoring of cell behaviors. Lab Chip 2012, 12, 3451-3457.

31. Gervais, T.; El-Ali, J.; Günther, A.; Jensen, K.F. Flow-induced deformation of shallow microfluidic channels. Lab Chip 2006, 6, 500-507.

32. Bryan, A.K.; Goranov, A.; Amon, A.; Manalis, S.R. Measurement of mass, density, and volume during the cell cycle of yeast. Proc. Natl. Acad. Sci. USA 2010, 107, 999-1004.

33. Ahmad, M.R.; Nakajima, M.; Kojima, S.; Homma, M.; Fukuda, T. The Effects of cell sizes, environmental conditions, and growth phases on the strength of individual W303 yeast cells inside ESEM. IEEE Trans. Nanobiosci. 2008, 7, 185-193.

34. Smith, A.E.; Zhang, Z.; Thomas, C.R.; Moxham, K.E.; Middelberg, A.P. The Mechanical properties of saccharomyces cerevisiae. Proc. Natl. Acad. Sci. USA 2000, 97, 9871-9874.

35. Stenson, J.D.; Thomas, C.R.; Hartley, P. Modelling the mechanical properties of yeast cells. Chem. Eng. Sci. 2009, 64, 1892-1903.

36. Stenson, J.D.; Hartley, P.; Wang, C.; Thomas, C.R. Determining the mechanical properties of yeast cell walls. Biotechnol. Prog. 2011, 27, 505-512.

37. Burg, T.P.; Godin, M.; Knudsen, S.M.; Shen, W.; Carlson, G.; Foster, J.S.; Babcock, K.; Manalis, S.R. Weighing of Biomolecules, Single Cells and Single Nanoparticles in Fluid. Nature 2007, 446, 1066-1069.

38. Lee, J.; Chunara, R.; Shen, W.; Payer, K.; Babcock, K.; Burg, T.P.; Manalis, S.R. Suspended microchannel resonators with piezoresistive sensors. Lab Chip 2011, 11, 645-651. 


\title{
Get to Understand More from Single-Cells: Current Studies of Microfluidic-Based Techniques for Single-Cell Analysis
}

\section{Shih-Jie Lo and Da-Jeng Yao}

\begin{abstract}
This review describes the microfluidic techniques developed for the analysis of a single cell. The characteristics of microfluidic (e.g., little sample amount required, high-throughput performance) make this tool suitable to answer and to solve biological questions of interest about a single cell. This review aims to introduce microfluidic related techniques for the isolation, trapping and manipulation of a single cell. The major approaches for detection in single-cell analysis are introduced; the applications of single-cell analysis are then summarized. The review concludes with discussions of the future directions and opportunities of microfluidic systems applied in analysis of a single cell.
\end{abstract}

Reprinted from Int. J. Mol. Sci. Cite as: Lo, S.-J.; Yao, D.-J. Get to Understand More from Single-Cells: Current Studies of Microfluidic-Based Techniques for Single-Cell Analysis. Int. J. Mol. Sci. 2015, 16, 16763-16777.

\section{Introduction}

A single cell is the smallest functional unit within an organism and maintains the functions of tissues through mutual cooperation. Biological research has extended from cell physiology and mechanics to gene expression. Accompanied by numerous achievements of biological research over hundreds of years, biology has progressed to becoming a data-rich subject; omic biology thus is integral to large-scale study (i.e., genome, proteome, connectome etc.). Apart from the rise of omic biology, in single-cell biology there have been investigations of the effect of one or a few genes in thousands of single cells over a long period of time. Single-cell biology utilizes speed-limited approaches (i.e., fluorescence in situ hybridization; FISH) to study functions in a single cell, whereas omic biology employs high-throughput approaches (i.e., whole genome sequencing) to collect much data rapidly. Note, omic biology passes over variations between cells. In recent years, molecular biotechnology and the related techniques have progressed toward quantitative analysis of a single cell. In this way, omic biology moves in the same direction as single-cell biology, namely studying many genes in many single cells [1]. No matter in which direction biological research evolves, single-cell analysis hence remains an important and fundamental topic in the biological field.

Although analysis of a single-cell plays an important role in biological search for an understanding of functions in an organism, analytical techniques face diverse challenges: (1) the analytical tool should work well with a single cell as the size of a cell is below the micrometer scale, and the target in a single cell of interest is thus on a sub-micrometer or even nanometer scale; (2) the analysis is conducted using little sample; (3) the analytical approach could be handled with high throughput so as to acquire sufficient data for significant statistics; and (4) the analytical tool is universally affordable for laboratories. Microfluidic systems thus conform to these requirements 
and have exhibited great performance in recent decades [2]. Microfluidic systems transport and manipulate a liquid volume ranging from microlitres to femtolitres in microfluidic channels of which the dimensions are on a micrometer scale. A living biological specimen has typically a liquid form; as a result microfluidics is favorable for single-cell analysis. Moreover, microfluidic chips are made of inexpensive materials; the chips can certainly be designed for high-throughput use. Microfluidic chips have hitherto been fabricated from silicon or glass, elastomer, thermosets, hydrogel, thermoplastics and paper [3]. Microfluidic chips are sometimes constituted as multiple layers of varied materials for a specific purpose [4,5], i.e., an elastomer bound to glass for a product with increased rigidity. Polydimethylsiloxane (PDMS) is an elastomer of one kind that has become a popular material for the fabrication of microfluidic chips because of its low cost, gas permeability and biocompatibility; most microfluidic devices related to biological research are consequently made of PDMS. In this review, we hence focus on the microfluidic-based techniques that have been applied in single-cell analysis and highlight some new developments.

\section{Isolation, Trapping and Manipulation of Single Cells}

Before initiating a single-cell analysis, cells of interest must be selected for further treatment. One advantage of a microfluidic device is that one can generate droplets with tiny volumes (nL to $\mathrm{fL)}$ as a separated environment for growth or chemical reaction of a single cell. Table 1 summarizes the microfluidic techniques for single-cell analysis and Figure 1 shows an overview of various approaches for the isolation, trapping and manipulation of a single cell. The concept of droplet-based analysis is similar to that of micro-wells, significantly decreasing the possibility of cross-contamination. The generated droplets are then selected with a flow cytometer. Flow cytometry is a historic and commonly used technique in biological laboratories for high-throughput sorting of cells of interest; it can be used for analysis of the cell properties according to various parameters, i.e., cell morphology, cell viability, protein expression etc. Accompanying the development of microfluids, flow cytometry is unrestricted to a cumbersome instrument, and can be operated in a pocket chip. The selection of cells of interest in a microfluidic-based flow cytometer can be classified into optical sorting and magnetic sorting. In optical sorting, the design of a flow cytometer is generally constituted by switching valves with a branched microfluidic channel. The switching valve can be mechanically based or bubble-based [6]. The width and height of a fluidic channel are sufficient for a single cell but not two cells concurrently passing through. The fluorescent signal of each cell is the selection criterion of optical sorting, which is excited by a microfluidic integrated laser system and emitted from fluorescein-linked antibodies or fluorescent probes; the cells of interest become guided to the reservoir via the function of a switching valve. This optical sorting is also named fluorescence-activated cell sorting (FACS) [7]. The cell selection of interest might thus be achieved with a flow cytometer via a specific antibody-antigen conjugation and labeling with a fluorescent probe. Secondly, according to the magnetic sorting approach, magnetic-affinity cell sorting (MACS) [8], also employs the specificity of an antibody-antigen conjugation, but the difference is a magnetic-bead-linked antibody rather than a fluorescein-linked one. To select a cell in magnetic sorting involves applying a magnetic field at a cell-collection reservoir, through which a fluid flows, to capture the magnetic-bead-labeled cells. A droplet-based 
microfluidic device with the function of flow cytometry can thus achieve single-cell analysis of various types [9]. A droplet-based microfluidic device can also be integrated with various sorting techniques, introduced in what follows, to select the target cells or compounds for analysis.

To date, varied trap techniques are commonly utilized and combined with microfluidic systems for observation or manipulation. If enduring manipulation is required, the trapped single cells also can be cultivated on a chip with nutrition supplied in a continuous flow. In a flow cytometer, the cells of interest are selected. In what follows we introduce the commonly used techniques of cell separation and manipulation for advanced analysis of single cells.

Table 1. Comparison of various microfluidic techniques for single-cell analysis.

\begin{tabular}{|c|c|c|c|}
\hline Approaches & Main Applications & Major Advantage & Major Disadvantage \\
\hline Droplet-based microfluidics & $\begin{array}{l}\text { - Single cell isolation } \\
\text { - Cell culture in droplet }\end{array}$ & $\begin{array}{l}\text { High throughput screening } \\
\text { for specific single-cells }\end{array}$ & $\begin{array}{l}\text { Challenge to encapsulate } \\
\text { single-cells in each droplet }\end{array}$ \\
\hline Hydrodynamic trap & $\begin{array}{l}\text { - Single cell isolation } \\
\text { - Cell culture on chip }\end{array}$ & $\begin{array}{l}\text { Multifunction in one } \\
\text { device }\end{array}$ & $\begin{array}{c}\text { Complicated fabricating } \\
\text { process }\end{array}$ \\
\hline Magnetic trap & - Specific cell trapping & $\begin{array}{c}\text { Efficient trapping of } \\
\text { labeled cells }\end{array}$ & $\begin{array}{c}\text { Requires antibodies or } \\
\text { primers for magnetic label }\end{array}$ \\
\hline Acoustic trap & - Cell manipulation & Good for cell positioning & $\begin{array}{c}\text { May have negative effect } \\
\text { to cells }\end{array}$ \\
\hline Dielectrophoretic trap & $\begin{array}{l}\text { - Cell manipulation } \\
\text { - Cell selection }\end{array}$ & $\begin{array}{c}\text { Easily select target cells via } \\
\text { alternating frequency of } \\
\text { AC }\end{array}$ & $\begin{array}{c}\text { Heat problem during } \\
\text { long-term manipulation }\end{array}$ \\
\hline Optical trap & $\begin{array}{l}\text { - Cell manipulation } \\
\text { - Cell mechanics }\end{array}$ & $\begin{array}{l}\text { Applicable in many } \\
\text { fields of study }\end{array}$ & $\begin{array}{c}\text { Requires expensive } \\
\text { optical system }\end{array}$ \\
\hline
\end{tabular}

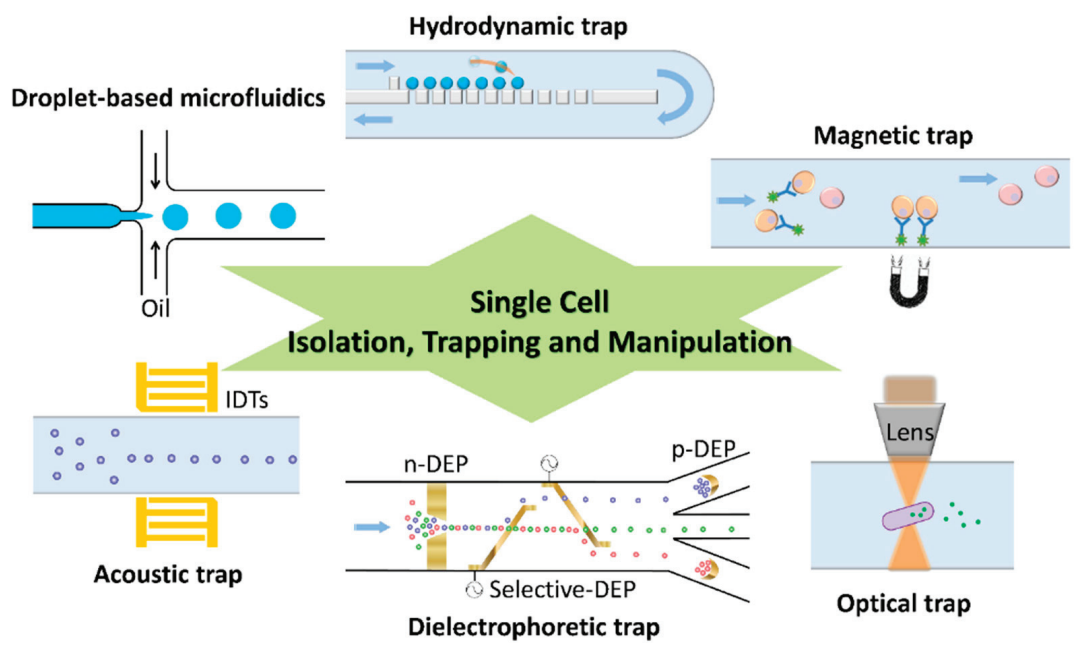

Figure 1. Schematic diagram of diverse approaches for the isolation, trapping and manipulation of single cells in a microfluidic device. 


\subsection{Hydrodynamic Trap}

Hydrodynamic trap utilizes specific microstructures and valves in a microfluidic channel to control the fluid flow so as to collect single cells without the use of other apparatus. This method is simple, but the fabrication of a microfluidic channel might be complicated because of microstructures. The structure of a microfluidic channel acts like a filter to trap cells within the fluid flow so as to acquire many single cells, but the number of cells lost is considerable, which should be considered in applications. Long et al. [10] fabricated a microfluidic chip containing two large feeding channels connected with multiple trapping or growth channels on a sub-micrometer scale. This ladder-like microfluidic chip was used to study the population of $E$. coli responding to dynamic changes in their environment that was achieved on varying the composition of growth media in feeding channels. Lin et al. [11] demonstrated sieve-like trap arrays in a microfluidic channel to trap and to position single cells on a glass substrate for their interactive study. Various paired configurations to trap cells were efficiently investigated and discussed in this work, providing an alternative approach for cell patterning. Secondly, there is another kind of hydrodynamic trap which employs the characteristics of fluidics via alternating the flow rate, causing either laminar flows or vortex flows, so as to achieve a specific purpose such as locating targets at the desired micro-structure. Sochol et al. [12] demonstrated a resettable hydrodynamic arraying system for trapping and releasing the target single cells. Although the performance of target trapping is important, the efficiency of target releasing is also a major concern in device development. In their work, the loading efficiency of the device was finally $99.8 \%$ and $78 \%$ for bead-based and cell-based experiments, accordingly. Wang et al. [13] developed a microfluidic hydrodynamic trapping system with the capability of long-term monitoring the cellular dynamics. The microfluidic device has a special bypass structure, which alternates the hydrodynamics in flow channel, and traps single-cells at the desired locations. The microfluidic trapping array has single cell trapping efficiency of $\sim 90 \%$ and used as a tool for evaluating the efficiency of chemotherapeutic reagents.

\subsection{Optical Trap}

Optical trap is also called optical tweezers, which is a highly precise technique for manipulating micro-scale objects. The optical trap utilizes laser beam to generate a force sub-pN in a microfluidic channel [14], which is sufficient to trap single cells. From a combination of an optical system and a microfluidic system, a new research field called optofluidics has emerged. On altering the focus of laser beams in a microfluidic channel, an object (i.e., blood cells, PS beads, etc.) can be manipulated according to its shape, size and refractive index. The cost of using an optical trap to separate single cells is great because of the optically controlled system to locate precisely the focus of laser beams. Moreover, prolonged manipulation in an optical trap might cause a problem because the cells become damaged through the heat generated from the laser energy. Liberale et al. [15] developed an integrated microfluidic device containing a micro-prism structure, which was fabricated with two-photon photolithography and allowed light from an optical fiber to trap a single cell. The integrated microfluidic device is capable of on-chip manipulation, Raman and fluorescence spectra 
of single cells. An optical trap has been developed to alter the shape of an aperture to improve the trapping efficiency, such as a rectangle, a double nanohole $(\mathrm{DNH})$ and a coaxial aperture. The DNH optical trap has been utilized to study protein-protein interaction [16] and protein-DNA interaction [17], and also to determine the size and concentration of nanoparticles in solution [18].

\subsection{Magnetic Trap}

The isolating technique based on magnetic force functions through an action of immunomagnetic labeling or a hybridization of a nucleic-acid probe modified with magnetic beads. The objects of interest contain antigens that can be recognized by specific antibodies; the antibodies are linked with dextran-coated magnetic particles. The magnetically labeled objects can hence be captured in a microfluidic device treated with a magnetic field. The separation can be implemented through positive selection (i.e., collect objects linked with magnetic beads) or negative selection (i.e., collect objects without magnetic beads linked). The only disadvantage is the specificity of a magnetic trap depending on the antibodies and the primer design. Lai et al. [19] developed a microfluidic chip integrated with a magnetic trap for the screening of aptamers specific to influenza A virus; the aptamer screening, also called systematic evolution of ligands and exponential enrichment (SELEX), was shortened to $60 \mathrm{~min}$ with this micro fluidic chip, to be compared with a conventional process that requires at least $160 \mathrm{~min}$. Chen et al. [20] developed a mobile magnetic trap array, which was integrated with a droplet-generating microfluidic device, to encapsulate magnetically selected single cells as a powerful analytical tool for a single cell. Nawarathna et al. [21] developed an integrated nanoscale magnetic trap within a plastic microfluidic device; the magnetic field gradients therein were significantly increased to trap magnetic beads efficiently.

\subsection{Dielectrophoretic Trap}

Dielectrophoresis (DEP) is a phenomenon that involves a motion of polarizable particles under a non-uniform electric field. The types of DEP can be briefly classified into positive DEP ( $p$-DEP) and negative DEP (n-DEP) [22,23], depending on the permittivity of the polarizable particles and the surrounding medium. When the permittivity of particles is greater than that of the medium, the particles have polarized opposite charges in the electric field; the particles move to the direction of a strong electric field, which is called p-DEP. n-DEP exhibits conversely that the particles move to the direction of a weak electric field when the permittivity of particles is less than that of a medium. The particles have polarized identical charges in the electric field because the polarized charges of the particles surface become greater than that of the particle inside. The electrical property of a particles surface and the interior is opposite as a result of the particles being induced with identical charges in the electric field. DEP is readily incorporated into a microfluidic device via a fabricated microelectrode. Cell patterning in microfluidic device is achievable via a DEP approach with an electrode array [24]. A dielectrophoretic trap has also a smaller heat problem than an optical trap during a protracted manipulation, because the heat generated is less and is removed adequately with the fluid flow. Bhattacharya et al. [25] employed insulator-based dielectrophoresis 
(iDEP) to trap single mammalian breast-cancer cells (MCF-7) from mixtures with mammalian peripheral blood mononuclear cells (PBMC). Simulation and experimental results of their tear-drop structure in a microfluidic channel indicate the weakly metastatic cancers cell (MCF-7) could also be selectively trapped from a mixture containing the highly invasive cancer cells (MDA-MB-231), so that this iDEP based microfluidic device might be applied as a diagnostic tool for breast cancer. Huang et al. [26] also used a DEP-integrated microfluidic device for single MCF-7 cell trapping, and investigated the effects on a trapped cell under a varied applied electric field, suggesting that an applied electric field less than $2 \mathrm{kV} / \mathrm{cm}$ was safe for cell viability. An alternative material, a carbon electrode, has been used as a function of electrode and insulator structure for DEP applications [27].

\subsection{Acoustic Trap}

A surface-acoustic wave can be used as a non-contact approach to sense a specific analyte or to confine single cells in a microfluidic channel. The principle of an acoustic trap is that an ultrasonic standing wave, produced with a pair of interdigital transducers (IDT) while applying electricity, traps or agglomerates cells. The IDT structure is deposited on a piezoelectric substrate in a parallel or orthogonal arrangement for varied cell patterning [28]. The negative effect of acoustic manipulation has been an issue and discussed [29,30], but little evidence verifies that exposure to ultrasound decreases the survival rate of cells. Chen et al. [31] developed a microfluidic device based on a standing surface-acoustic wave (SSAW) for continuous enrichment of a limited cell sample. The limited sample can be concentrated within the standing waves in a microfluidic channel and be eventually collected for further cellular study. The systems of acoustic trap and optical tweezers are currently combined as optoacoustic tweezers in a microfluidic device with the characteristics of biocompatibility and ease of fabrication for dynamically concentrating and patterning particles and cells [32].

\section{Single-Cell Analysis in a Microfluidic Device}

As varied techniques to trap a single are applicable in a microfluidic device, techniques of microanalysis are equally important in the analysis of a single cell. Electrophoresis is a commonly used technique to separate small molecules via varied electrophoretic mobility of charged molecules in an electric field. A major difference between electrophoresis and dielectrophoresis (DEP) depends on the objects being charged, or not. The separation principle of electrophoresis is based on the electric charge of an analyte, whereas DEP is based on an induced dipole inside an analyte. Accompanying the development of microfluidics, electrophoresis can be implemented within a narrow capillary, named capillary electrophoresis (CE), and be integrated to the microfluidic chip. Because of a small amount of single-cell analysis, CE has been applied as a treatment before an analysis and has the advantage of concentrating the analyte for the next step in the analysis.

In addition to lyse cells for $\mathrm{CE}$ approach, various non-invasive methods are applicable for live-cell detection. Investigation of live-cells has more restrictions than analyzing cell lysates 
because of the cell structure (e.g., cell membrane or nuclear membrane) that hinders the interactions between the labeling probes and targets of cells. Optical measurement is the most used non-invasive method for biological research; furthermore, fluorescence detection currently is simple and convenient for many kinds of research application because of the plentiful commercialized fluorescent probes. Fluorescent proteins, nanoparticles and quantum dots all can be utilized as the fluorescent source of probes, the specificity of which depend on the antibodies or DNA sequence (aptamers). Li et al. [33] developed a highly sensitive detection system for membrane protein of living single-cells by employing aptamer and enzyme assisted fluorescence amplification, which successfully carried out high throughput single-cell analysis of the lowabundance biomarker (PTK7). In what follows we introduce the major detection approaches for single-cell analysis (Figure 2).

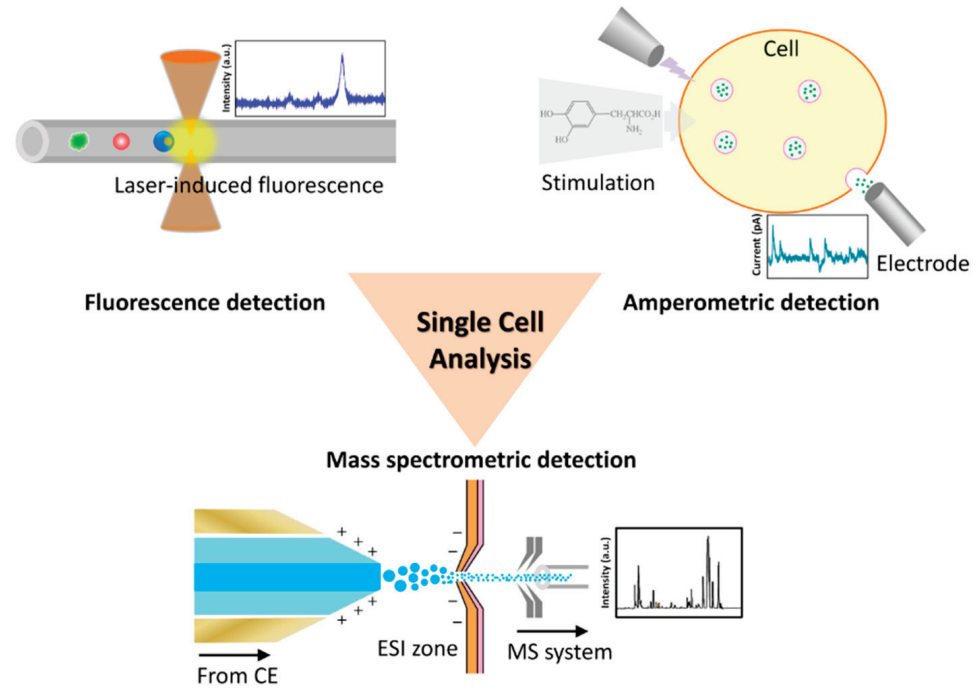

Figure 2. Schematic diagram of major approaches for the analysis of single cells with a microfluidic device.

\subsection{Fluorescence Detection}

Rough information about cellular contents can be acquired by lysing a trapped single cell and performing capillary electrophoresis, but such rough information to understand an organism in detail is limited. Capillary electrophoresis and laser-induced fluorescence (CE-LIF) provide scientists with more sub-cellular information about the interior of the cells. LIF employs a laser to excite fluorescence from a specific molecular moiety, and has been integrated with $\mathrm{CE}$ in a microfluidic device that provides a powerful technique with great sensitivity and a small detection limit for use in single-cell analysis. The fluorescein tagged substance is an analytical target of the LIF approach; the structure of the compound in the cell that signals transmission typically contains aromatic rings exhibiting self-fluorescence that is an effective candidate for LIF detection. 
Keithley et al. [34] developed a capillary electrophoresis system with a three-color laser-induced fluorescence detector to measure BODIPY fluorophores conjugated glycophingolipids. Three fluorophores (BODIPY-FL, BODIPY-TMR and BODIPY-650/665) were prepared in a chemoenzymatic synthesis and excited with diode-pumped solid-state lasers and a diode laser at wavelengths 473, 532 and $633 \mathrm{~nm}$ sequentially. Neuronal-like dPC12 cells were incubated with the fluorophores; their metabolic products were studied with the three-color CE-LIF system. Metto et al. [35] demonstrated an integrated microfluidic device to measure nitrogen oxide (NO) produced in single T-lymphocytes (Jurkat cells). The functions of cell transport, lysis, injection, electrophoretic separation and fluorescence detection were integrated in that microfluidic device. Two fluorescent probes, 4-amino-5-methylamino-2',7'-difluorofluorescein diacetate (DAFFM DA) and 6-carboxyfluorescein diacetate (6-CFDA), were used to label NO and Jurkat cells sequentially. NO production in the cells can be stimulated on applying lipopolysaccharide (LPS) via inducible nitrogen oxide synthase (iNOS) in immune cells. The ultimate results of NO measurement from single-cell analysis compared well with the bulk cell level. Ban et al. employed a CE-LIF system to detect micro-RNA in cardiomyoblast cells [36] and lung-cancer cell lines [37]. Micro-RNA was captured via hybridization with DNA probes labeled with 6-FAM- or Cy5 in a separate sequence, and detected with a dual laser system. Their results showed that CE-LIF could be completed within $13 \mathrm{~min}$ and evaluated several endogenous miRNA.

\subsection{Amperometric Detection}

Although much subcellular information can be acquired via fluorescently based single-cell analysis, such as with fluorescein conjugated antibodies to capture proteins or fluorescein labeled probes to hybridize nucleic acids (DNA, mRNA), another approach that applied amperometry has been used to study specific proteins, especially in neurobiological research. Amperometry combined with capillary electrophoresis for electrochemical analysis of single cells becomes an effective tool to analyze chemical messengers (e.g., neurotransmitters, neuropeptides and neurohormones) in neurons; this method is also called capillary electrophoresis with electrochemical detection (CE-ED) [38]. The microfluidic device is integrated with an amperometric detecting system. After applying a stimulation of a trapped neuron (e.g., electric stimulation or chemical stimulation), chemical messengers in vesicles are released via exocytosis of a neuron of which the response can be measured. Moreover, the released chemical compounds can be collected concurrently in a microfluidic channel. Omiatek et al. [39] used this technique to measure the total vesicular content from single neuronal cells (PC12 cells), and found that a vesicle released only $40 \%$ of their transmitter load from a comparison of the single neuron measurement and a cell-free mode. They found also a phenomenon in which the release of a vesicular neurotransmitter and a hormone is not certainly all or none. In addition, the background noise decides the limit of amperometric detection, especially in a biosensor application. Larsen et al. [40] investigated the current noise caused by various electrode materials with varied capacitive properties, and concluded that low- capacitive materials and small electrodes have a small current noise, which benefits the design of a low-noise amperometric sensing device. Other than lowering the current noise, they investigated also the physical and electrochemical properties of poly(3,4-ethylenedioxythiophene):tosylate (PEDOT:tosylate) as a microelectrode 
material for neurochemical detection [41]. From the results, the capacitance of this conductive polymer is greater than of other thin-film materials, which limits the low-noise amperometric sensing, but this shortage can be overcome through sufficiently small fabrication. The neuron transmitter release of PC12 cells was measured with this chip-based device with microelectrodes made of PEDOT:tosylate that have the advantages of cheap and easy fabrication in all polymer devices.

\subsection{Mass Spectrometric Detection}

Mass spectrometer (MS) is a powerful tool to measure chemical components, whether used for qualitative or quantitative purpose, and is capable of providing an analysis with information about concentration, chemical structure and elemental composition. As the great sensitivity of a MS combined with $\mathrm{CE}$ has rapid separation and efficiency, the CE-MS analytical method becomes a suitable approach for single-cell analysis. The $\mathrm{CE}$ within a microfluidic device functions to selectively collect components of interest in a single cell and to concentrate the components before analyzing via a MS; the CE processed sampling approach could significantly simplify analyses entering a MS, in order to achieve a reduction of noisy signals from undesired components via the mass spectrometric analysis. With a period of CE-MS technological development, various instruments have been used for sample-ionization interfacing between a CE and a MS, such as electrospray ionization (ESI), matrix-assisted laser desorption or ionization (MALDI), laser-ablation electrospray ionization (LAESI) or inductively coupled plasma (ICP). A compatible ionizing interface is important because it affects the sensitivity of CE-MS [42]. To date, ESI and MALDI are the most popular methods for a sample-ionizing interface, but they both have advantages and disadvantages. For instance, ESI is suitable for online interfacing of a microfluidic device to a MS because of the compatible small flow rate [43]. ESI generates highly charged ions directly from a micro-fluid, which assists easy coupling between a CE and a MS, but ion suppression due to the large concentration of salts within samples becomes a challenge of analysis. In contrast, MALDI has a greater tolerance to salts, but matrix ions of MALDI limit the spectral analysis to molecular mass less than $500 \mathrm{Da}$. Aerts et al. [44] applied entire-cell patch-clamp recording and a CE-MS to establish the cytoplasm metabolomics of a single neuron. The physiological activity of glutamatergic thalamocortical neurons of a rat were recorded via the entire-cell patch-clamp technique, of which the metabolomics were analyzed via a CE-MS. Approximately 60 metabolites were detected and determined via a CE-MS from a tiny volume $(\sim 3 \mathrm{pL})$ of neuronal cytoplasm. This combined technique provides a new measurement tool to study the changes of cellular metabolome-related neuronal activity. Mellors et al. [45] developed an integrated microfluidic device that used a CE and an ESI-MS to analyze single cells automatically in real time. Human erythrocytes were lysed with this microfluidic device, of which the components were separated with the function of CE. The separated components were then ionized via an electrospray emitter and characterized in a MS. This device can verify the heme group and subunits of hemoglobin from individual erythrocytes with a throughput rate 12 cells per minute. The sensitivity of this device can ultimately be improved in optimizing the channel dimensions to decrease the possible flow rate. Smith et al. [46] developed an ESI-MS- integrated droplet-based microfluidic device for study of single cells with high 
throughput. Four populations of droplets, containing cytochrome $\mathrm{C}, \alpha$-chymotrypsinogen A, carbonic anhydrase or chicken lysozyme, were generated with a surfactant-stabilized reagent in a microfluidic device. The droplets containing sub-femtomolar quantities of an analyte were collected and then reinjected into the main channel of a microfluidic device for further characterization via an ESI-MS. This technique provides an alternative approach for analysis of single cells with high throughput.

\section{Application Summary of Single-Cell Analysis}

To date, microfluidic-based single-cell analysis has been applied in cellular research of diverse aspects and with multiple functions, for example, polymerase chain reaction (PCR) for gene analysis or cell differentiation for regenerative medicine. As mentioned at the beginning of this review, single-cell biology begins to investigate the functional mechanism of an organism from a single cell, the goal of which is the same as omic biology and provides more information about cellular heterogeneity. Many data of single-cell analysis can now be catalogued as single-cell genomics, single-cell transcriptomics and single-cell proteomics. Single-cell genomics can be established using single-cell qPCR [47], nanopore-based DNA sequencing [48] etc., and provides researchers a complete understanding in order to verify whether a specific gene is constantly expressed in the same cell population or selectively expressed. Single-cell transcriptomics is thus generally established using a reverse transcriptase polymerase chain reaction (RT-PCR) [49,50], real-time PCR [51] or transcriptome sequencing (RNA-Seq) [52]. The study of single-cell transcriptomics provides intracellular variability of RNA profiles under varied environmental conditions. Compared with the omic biology of nucleic-acid-based methods, the construction of single-cell proteomics is much more difficult than single-cell genomics and transcriptomics because the small amount of protein in a single cell requires highly sensitive detection. A CE-integrated microfluidic device combined with MS-related techniques enables the convenient establishment of single-cell proteomics. The single-cell proteomics helps researchers in studying diverse post-translational modifications [53], translocations [54], and activity-correlating protein conformations [55]. Besides the above mentioned omic biology, epigenomics currently is a frontier in single-cell analysis [56]. Epigenomics investigates epigenetic modulations (e.g., DNA methylation or histone modification) of DNA, which is heritable and affects gene expressions in each single cell, although these single-cells have the same DNA sequence. Various bio-techniques now can be applied in establishing epigenomics [57], such as Chromatin immunoprecipitation followed by sequencing (ChIP-seq) [58] or bisulfite sequencing (BS-seq) [59], which have been used for locating histone modification.

Although the literature about microfluidic-based single-cell analysis is enormous, the contributions that matter most are in fundamental cellular research to provide new insights into the mechanisms of life. Single-cell analysis applications are now moving in the direction of diagnostic use and personalized medicine. The detection of circulating tumor cells (CTC) is a useful example of using a microfluidic device for diagnostic use. CTC are generated on metastasis of a primary tumor and are found in the peripheral blood from cancer patients. The variant number of CTC indicates the severity of the cancer and reducing it represents success of cancer therapies. The challenge of CTC detection is that CTC are rare in a heterogeneous sample (blood); high throughput and sensitive techniques are needed. A microfluidic device thus becomes an ideal tool 
to capture, separate, count and further analyze CTC efficiently [60-63]. Drug discovery for personalized medicine is also a prominent issue within single-cell analysis, to understand the mechanism of a drug-treated single cell, which exhibits a complicated response (i.e., gene expression, metabolic alteration). The single-cell transcriptome analysis can provide a variation of RNA profiles after a drug treatment; the proteomic analysis can rapidly screen the candidates of antigen-specific antibodies for potential drug use [64].

\section{Conclusions}

In summary, various techniques are now available for the analysis of a single cell; with the aid of these techniques, many biological questions can be answered. A microfluidic device is perhaps now a suitable technique for single-cell analysis because a microfluidic system can be manipulated with high throughput, and the amount of sample from a single cell is limited. A microfluidic device might, however, be replaced with the further development of tools through the future efforts of physicists and engineers to answer other interesting biological questions. Although devices ready to use for diagnosis at a point of care are rare at present, most techniques have been applied in basic research. We believe that from the combination of the versatile design of microfluidic devices, flexible choice of analytical technique and increased knowledge, a portable device for personalized medical use can ultimately emerge.

\section{Acknowledgments}

The authors would like to thank the financial support from National Science Council of Taiwan under Contract No. NSC 101-2221-E-007-099-MY3.

\section{Conflicts of Interest}

The authors declare no conflict of interest.

\section{References}

1. Junker, J.P.; van Oudenaarden, A. Every cell is special: Genome-wide studies add a new dimension to single-cell biology. Cell 2014, 157, 8-11.

2. Yin, H.B.; Marshall, D. Microfluidics for single cell analysis. Curr. Opin. Biotechnol. 2012, 23, 110-119.

3. Ren, K.N.; Zhou, J.H.; Wu, H.K. Materials for microfluidic chip fabrication. Acc. Chem. Res. 2013, 46, 2396-2406.

4. Villar, G.; Graham, A.D.; Bayley, H. A tissue-like printed material. Science 2013, 340, 48-52.

5. Chen, C.C.; Chen, Y.A.; Liu, Y.J.; Yao, D.J. A multilayer concentric filter device to diminish clogging for separation of particles and microalgae based on size. Lab Chip 2014, 14, 1459-1468. 
6. Mosadegh, B.; Kuo, C.H.; Tung, Y.C.; Torisawa, Y.S.; Bersano-Begey, T.; Tavana, H.; Takayama, S. Integrated elastomeric components for autonomous regulation of sequential and oscillatory flow switching in microfluidic devices. Nat. Phys. 2010, 6, 433-437.

7. Cho, S.H.; Godin, J.M.; Chen, C.H.; Qiao, W.; Lee, H.; Lo, Y.H. Review Article: Recent advancements in optofluidic flow cytometer. Biomicrofluidics 2010, 4, 43001.

8. Gao, Y.; Li, W.J.; Pappas, D. Recent advances in microfluidic cell separations. Analyst 2013, $138,4714-4721$.

9. Wang, B.L.; Ghaderi, A.; Zhou, H.; Agresti, J.; Weitz, D.A.; Fink, G.R.; Stephanopoulos, G. Microfluidic high-throughput culturing of single cells for selection based on extracellular metabolite production or consumption. Nat. Biotechnol. 2014, 32, 473-478.

10. Long, Z.; Nugent, E.; Javer, A.; Cicuta, P.; Sclavi, B.; Lagomarsino, M.C.; Dorfman, K.D. Microfluidic chemostat for measuring single cell dynamics in bacteria. Lab Chip 2013, 13, 947-954.

11. Lin, L.Y.; Chu, Y.S.; Thiery, J.P.; Lim, C.T.; Rodriguez, I. Microfluidic cell trap array for controlled positioning of single cells on adhesive micropatterns. Lab Chip 2013, 13, 714-721.

12. Sochol, R.D.; Dueck, M.E.; Li, S.; Lee, L.P.; Lin, L.W. Hydrodynamic resettability for a microfluidic particulate-based arraying system. Lab Chip 2012, 12, 5051-5056.

13. Wang, Y.; Tang, X.L.; Feng, X.J.; Liu, C.; Chen, P.; Chen, D.J.; Liu, B.F. A microfluidic digital single-cell assay for the evaluation of anticancer drugs. Anal. Bioanal. Chem. 2015, 407, 1139-1148.

14. Ramser, K.; Hanstorp, D. Optical manipulation for single-cell studies. J. Biophotonics 2010, 3 , 187-206.

15. Liberale, C.; Cojoc, G.; Bragheri, F.; Minzioni, P.; Perozziello, G.; la Rocca, R.; Ferrara, L.; Rajamanickam, V.; di Fabrizio, E.; Cristiani, I. Integrated microfluidic device for single-cell trapping and spectroscopy. Sci. Rep. 2013, 3, 1258.

16. Zehtabi-Oskuie, A.; Jiang, H.; Cyr, B.R.; Rennehan, D.W.; Al-Balushi, A.A.; Gordon, R. Double nanohole optical trapping: Dynamics and protein-antibody co-trapping. Lab Chip 2013, 13, 2563-2568.

17. Kotnala, A.; Gordon, R. Double nanohole optical tweezers visualize protein p53 suppressing unzipping of single DNA-hairpins. Biomed. Opt. Express 2014, 5, 1886-1894.

18. Kotnala, A.; DePaoli, D.; Gordon, R. Sensing nanoparticles using a double nanohole optical trap. Lab Chip 2013, 13, 4142-4146.

19. Lai, H.C.; Wang, C.H.; Liou, T.M.; Lee, G.B. Influenza A virus-specific aptamers screened by using an integrated microfluidic system. Lab Chip 2014, 14, 2002-2013.

20. Chen, A.; Byvank, T.; Chang, W.J.; Bharde, A.; Vieira, G.; Miller, B.L.; Chalmers, J.J.; Bashir, R.; Sooryakumar, R. On-chip magnetic separation and encapsulation of cells in droplets. Lab Chip 2013, 13, 1172-1181.

21. Nawarathna, D.; Norouzi, N.; McLane, J.; Sharma, H.; Sharac, N.; Grant, T.; Chen, A.; Strayer, S.; Ragan, R.; Khine, M. Shrink-induced sorting using integrated nanoscale magnetic traps. Appl. Phys. Lett. 2013, 102, 63504. 
22. Jubery, T.Z.; Srivastava, S.K.; Dutta, P. Dielectrophoretic separation of bioparticles in microdevices: A review. Electrophoresis 2014, 35, 691-713.

23. Gossett, D.R.; Weaver, W.M.; Mach, A.J.; Hur, S.C.; Tse, H.T.K.; Lee, W.; Amini, H.; di Carlo, D. Label-free cell separation and sorting in microfluidic systems. Anal. Bioanal. Chem. 2010, 397, 3249-3267.

24. Taff, B.M.; Voldman, J. A scalable addressable positive-dielectrophoretic cell-sorting array. Anal. Chem. 2005, 77, 7976-7983.

25. Bhattacharya, S.; Chao, T.C.; Ariyasinghe, N.; Ruiz, Y.; Lake, D.; Ros, R.; Ros, A. Selective trapping of single mammalian breast cancer cells by insulator-based dielectrophoresis. Anal. Bioanal. Chem. 2014, 406, 1855-1865.

26. Huang, C.J.; Liu, C.X.; Stakenborg, J.L.T.; Lagae, L. Single cell viability observation in cell dielectrophoretic trapping on a microchip. Appl. Phys. Lett. 2014, 104, 013703.

27. Martinez-Duarte, R.; Camacho-Alanis, F.; Renaud, P.; Ros, A. Dielectrophoresis of lambda-DNA using 3D carbon electrodes. Electrophoresis 2013, 34, 1113-1122.

28. Shi, J.J.; Ahmed, D.; Mao, X.; Lin, S.C.S.; Lawit, A.; Huang, T.J. Acoustic tweezers: Patterning cells and microparticles using standing surface acoustic waves (SSAW). Lab Chip 2009, 9, 2890-2895.

29. Evander, M.; Johansson, L.; Lilliehorn, T.; Piskur, J.; Lindvall, M.; Johansson, S.; Almqvist, M.; Laurell, T.; Nilsson, J. Noninvasive acoustic cell trapping in a microfluidic perfusion system for online bioassays. Anal. Chem. 2007, 79, 2984-2991.

30. Hultstrom, J.; Manneberg, O.; Dopf, K.; Hertz, H.M.; Brismar, H.; Wiklund, M. Proliferation and viability of adherent cells manipulated by standing-wave ultrasound in a microfluidic chip. Ultrasound Med. Biol. 2007, 33, 145-151.

31. Chen, Y.; Li, S.; Gu, Y.; Li, P.; Ding, X.; Wang, L.; McCoy, J.P.; Levine, S.J.; Huang, T.J. Continuous enrichment of low-abundance cell samples using standing surface acoustic waves (SSAW). Lab Chip 2014, 14, 924-930.

32. Xie, Y.L.; Zhao, C.L.; Zhao, Y.H.; Li, S.X.; Rufo, J.; Yang, S.K.; Guo, F.; Huang, T.J. Optoacoustic tweezers: A programmable, localized cell concentrator based on opto-thermally generated, acoustically activated, surface bubbles. Lab Chip 2013, 13, 1772-1779.

33. Li, L.; Wang, Q.; Feng, J.; Tong, L.L.; Tang, B. Highly sensitive and homogeneous detection of membrane protein on a single living cell by aptamer and nicking enzyme assisted signal amplification based on microfluidic droplets. Anal. Chem. 2014, 86, 5101-5107.

34. Keithley, R.B.; Rosenthal, A.S.; Essaka, D.C.; Tanaka, H.; Yoshimura, Y.; Palcic, M.M.; Hindsgaul, O.; Dovichi, N.J. Capillary electrophoresis with three-color fluorescence detection for the analysis of glycosphingolipid metabolism. Analyst 2013, 138, 164-170.

35. Metto, E.C.; Evans, K.; Barney, P.; Culbertson, A.H.; Gunasekara, D.B.; Caruso, G.; Huvey, M.K.; da Silva, J.A.F.; Lunte, S.M.; Culbertson, C.T. An integrated microfluidic device for monitoring changes in nitric oxide production in single T-lymphocyte (jurkat) cells. Anal. Chem. 2013, 85, 10188-10195.

36. Ban, E.; Chae, D.K.; Song, E.J. Determination of micro-RNA in cardiomyoblast cells using CE with LIF detection. Electrophoresis 2013, 34, 598-604. 
37. Ban, E.; Chae, D.K.; Song, E.J. Simultaneous detection of multiple microRNAs for expression profiles of microRNAs in lung cancer cell lines by capillary electrophoresis with dual laser-induced fluorescence. J. Chromatogr. A 2013, 1315, 195-199.

38. Omiatek, D.M.; Cans, A.S.; Heien, M.L.; Ewing, A.G. Analytical approaches to investigate transmitter content and release from single secretory vesicles. Anal. Bioanal. Chem. 2010, 397, 3269-3279.

39. Omiatek, D.M.; Dong, Y.; Heien, M.L.; Ewing, A.G. Only a fraction of quantal content is released during exocytosis as revealed by electrochemical cytometry of secretory vesicles. ACS Chem. Neurosci. 2010, 1, 234-245.

40. Larsen, S.T.; Heien, M.L.; Taboryski, R. Amperometric noise at thin film band electrodes. Anal. Chem. 2012, 84, 7744-7749.

41. Larsen, S.T.; Vreeland, R.F.; Heien, M.L.; Taboryski, R. Characterization of poly(3,4ethylenedioxythiophene):tosylate conductive polymer microelectrodes for transmitter detection. Analyst 2012, 137, 1831-1836.

42. Kleparnik, K. Recent advances in the combination of capillary electrophoresis with mass spectrometry: From element to single-cell analysis. Electrophoresis 2013, 34, 70-85.

43. Gao, D.; Liu, H.X.; Jiang, Y.Y.; Lin, J.M. Recent advances in microfluidics combined with mass spectrometry: Technologies and applications. Lab Chip 2013, 13, 3309-3322.

44. Aerts, J.T.; Louis, K.R.; Crandall, S.R.; Govindaiah, G.; Cox, C.L.; Sweedler, J.V. Patch clamp electrophysiology and capillary electrophoresis-mass spectrometry metabolomics for single cell characterization. Anal. Chem. 2014, 86, 3203-3208.

45. Mellors, J.S.; Jorabchi, K.; Smith, L.M.; Ramsey, J.M. Integrated microfluidic device for automated single cell analysis using electrophoretic separation and electrospray ionization mass spectrometry. Anal. Chem. 2010, 82, 967-973.

46. Smith, C.A.; Li, X.; Mize, T.H.; Sharpe, T.D.; Graziani, E.I.; Abell, C.; Huck, W.T.S. Sensitive, high throughput detection of proteins in individual, surfactant-stabilized picoliter droplets using nanoelectrospray ionization mass spectrometry. Anal. Chem. 2013, 85, 3812-3816.

47. Livak, K.J.; Wills, Q.F.; Tipping, A.J.; Datta, K.; Mittal, R.; Goldson, A.J.; Sexton, D.W.; Holmes, C.C. Methods for qPCR gene expression profiling applied to 1440 lymphoblastoid single cells. Methods 2013, 59, 71-79.

48. Min, S.K.; Kim, W.Y.; Cho, Y.; Kim, K.S. Fast DNA sequencing with a graphene-based nanochannel device. Nat. Nanotechnol. 2011, 6, 162-165.

49. Petriv, O.I.; Kuchenbauer, F.; Delaney, A.D.; Lecault, V.; White, A.; Kent, D.; Marmolejo, L.; Heuser, M.; Berg, T.; Copley, M.; et al. Comprehensive microRNA expression profiling of the hematopoietic hierarchy. Proc. Natl. Acad. Sci. USA 2010, 107, 15443-15448.

50. White, A.K.; VanInsberghe, M.; Petriv, O.I.; Hamidi, M.; Sikorski, D.; Marra, M.A.; Piret, J.; Aparicio, S.; Hansen, C.L. High-throughput microfluidic single-cell RT-qPCR. Proc. Natl. Acad. Sci. USA 2011, 108, 13999-14004. 
51. Sanchez-Freire, V.; Ebert, A.D.; Kalisky, T.; Quake, S.R.; Wu, J.C. Microfluidic single-cell real-time PCR for comparative analysis of gene expression patterns. Nat. Protoc. 2012, 7 , 829-838.

52. Streets, A.M.; Zhang, X.N.; Cao, C.; Pang, Y.H.; Wu, X.L.; Xiong, L.; Yang, L.; Fu, Y.S.; Zhao, L.; Tang, F.C.; et al. Microfluidic single-cell whole-transcriptome sequencing. Proc. Natl. Acad. Sci. USA 2014, 111, 7048-7053.

53. Faley, S.L.; Copland, M.; Reboud, J.; Cooper, J.M. Cell chip array for microfluidic proteomics enabling rapid in situ assessment of intracellular protein phosphorylation. Biomicrofluidics 2011, 5, 24106.

54. Zhan, Y.H.; Martin, V.A.; Geahlen, R.L.; Lu, C. One-step extraction of subcellular proteins from eukaryotic cells. Lab Chip 2010, 10, 2046-2048.

55. Bockenhauer, S.; Furstenberg, A.; Yao, X.J.; Kobilka, B.K.; Moerner, W.E. Conformational dynamics of single $\mathrm{G}$ protein-coupled receptors in solution. J. Phys. Chem. B 2011, 115, 13328-13338.

56. Bheda, P.; Schneider, R. Epigenetics reloaded: The single-cell revolution. Trends Cell Biol. 2014, 24, 712-723.

57. Hyun, B.R.; McElwee, J.L.; Soloway, P.D. Single molecule and single cell epigenomics. Methods 2015, 72, 41-50.

58. Giannopoulou, E.G.; Elemento, O. Inferring chromatin-bound protein complexes from genome-wide binding assays. Genome Res. 2013, 23, 1295-1306.

59. Brinkman, A.B.; Gu, H.C.; Bartels, S.J.J.; Zhang, Y.Y.; Matarese, F.; Simmer, F.; Marks, H.; Bock, C.; Gnirke, A.; Meissner, A.; et al. Sequential ChIP-bisulfite sequencing enables direct genome-scale investigation of chromatin and DNA methylation cross-talk. Genome Res. 2012, 22, $1128-1138$.

60. Thege, F.I.; Lannin, T.B.; Saha, T.N.; Tsai, S.; Kochman, M.L.; Hollingsworth, M.A.; Rhim, A.D.; Kirby, B.J. Microfluidic immunocapture of circulating pancreatic cells using parallel EpCAM and MUC1 capture: Characterization, optimization and downstream analysis. Lab Chip 2014, 14, 1775-1784.

61. Bichsel, C.A.; Gobaa, S.; Kobel, S.; Secondini, C.; Thalmann, G.N.; Cecchini, M.G.; Lutolf, M.P. Diagnostic microchip to assay 3D colony-growth potential of captured circulating tumor cells. Lab Chip 2012, 12, 2313-2316.

62. Ozkumur, E.; Shah, A.M.; Ciciliano, J.C.; Emmink, B.L.; Miyamoto, D.T.; Brachtel, E.; Yu, M.; Chen, P.I.; Morgan, B.; Trautwein, J.; et al. Inertial focusing for tumor antigen-dependent and -independent sorting of rare circulating tumor cells. Sci. Transl. Med. 2013, 5, 179ra47.

63. Powell, A.A.; Talasaz, A.H.; Zhang, H.Y.; Coram, M.A.; Reddy, A.; Deng, G.; Telli, M.L.; Advani, R.H.; Carlson, R.W.; Mollick, J.A.; et al. Single cell profiling of circulating tumor cells: Transcriptional heterogeneity and diversity from breast cancer cell lines. PLoS ONE 2012, 7, e33788.

64. Golkowski, M.; Brigham, J.L.; Perera, B.G.K.; Romano, G.S.; Maly, D.J.; Ong, S.E. Rapid profiling of protein kinase inhibitors by quantitative proteomics. Medchemcomm 2014, 5, 363-369. 


\title{
Single Cell Electrical Characterization Techniques
}

\section{Muhammad Asraf Mansor and Mohd Ridzuan Ahmad}

\begin{abstract}
Electrical properties of living cells have been proven to play significant roles in understanding of various biological activities including disease progression both at the cellular and molecular levels. Since two decades ago, many researchers have developed tools to analyze the cell's electrical states especially in single cell analysis (SCA). In depth analysis and more fully described activities of cell differentiation and cancer can only be accomplished with single cell analysis. This growing interest was supported by the emergence of various microfluidic techniques to fulfill high precisions screening, reduced equipment cost and low analysis time for characterization of the single cell's electrical properties, as compared to classical bulky technique. This paper presents a historical review of single cell electrical properties analysis development from classical techniques to recent advances in microfluidic techniques. Technical details of the different microfluidic techniques are highlighted, and the advantages and limitations of various microfluidic devices are discussed.
\end{abstract}

Reprinted from Int. J. Mol. Sci. Cite as: Mansor, M.A.; Ahmad, M.R. Single Cell Electrical Characterization Techniques. Int. J. Mol. Sci. 2015, 16, 12686-12712.

\section{Introduction}

Study of the cell has emerged as a distinct new field, and acknowledged to be one of the fundamental building blocks of life. Moreover, the cells have unique biophysical and biochemical properties to maintain and sense the physiological surrounding environment to fulfill its specific functions [1,2]. Cellular biophysical properties analysis, such as the electrical, mechanical, optical and thermal characterization of cells, provides critical knowledge to diagnostics, clinical science and pharmaceutical industry [1]. Biophysical properties of cells provide early signals of disease or abnormal condition to the human body, which make it them valuable as potential markers for identifying cancers [3-7], bacteria [8-10], toxin detection [11] and the status of tissues [12,13]. Furthermore rapid growing technologies (e.g., conventional patch-clamp, dual nanoprobe-ESEM (environmental scanning electron microscope) and microfluidics) to investigate the biophysical properties of cells have been invented and developed by the researchers in the last decades. The technologies are continually improved make substantial contributions to biology and the clinical research community $[14,15]$.

Single cell analysis (SCA) has become a trend and major topic to engineers and scientists in the last 20 years to develop the experimental tools and technologies able to carry out single cell measurement. In addition, in depth analysis and more fully described activities of cell differentiation and cancer can only be accomplished with single cell analysis [16]. In conventional methods of cellular analysis, population based studies have been utilized for cellular processes such as metabolism, motility, cell growth and proliferation. Population methods use averages of cell properties to measure and predict the biophysical and biochemical parameters of cell. However, this method suffers from inaccurate measurements and often overlooks the essential information 
available in the cell due to the heterogeneity of cells (e.g., specific gene expression levels) [17]. For this reason, single cell studies have been emphasized to provide biologists and scientists to peer into the molecular machinery of individual cells. Single cell analysis has also been essential to our understanding of some fundamental questions, such as what makes single cells different biophysically, biochemically and functionally. Single cell analysis has been a key in probing of cancer $[4,18]$, and thus helps doctors to develop a prognosis and design a treatment plan for particular patients.

Electrical properties of cells provide some insight and vital information to aid the understanding of complex physiological states of the cell. Cells that experience abnormalities or are infected by bacteria may have altered ion channel activity [19], cytoplasm conductivity and resistance [20,21] and deformability [22]. For instance, red blood cells (RBCs) infected by Plasmodium falciparum, which cause malaria in humans, reduce deformability of the $\mathrm{RBC}$ by producing cytoadherence-related neoantigens that increase the rigidity and internal viscosity of the cytomembrane [23,24]. Each RBC which experiences the deformation process, has difference resistance, where the average resistance value of normal RBCs and rigidified RBCs are 14.2 and 19.6 $\Omega$ respectively [23]. Since electrical properties of cells have several advantages in cells analysis such as counting, separating, trapping and characterizing of single cell, development of suitable devices for single cell electrical analysis in term of accuracy prediction, portable, and user friendly are very important. In this review, we present an overview of classical technique and microfluidics technique in single cell electrical properties analysis.

\section{Classical Platforms}

The classical technique for a cell's electrical properties analysis was originated in 1791, when Luigi Galvani conducted the first experiment for measuring electrical activity in animals, which is evoking muscular contractions in frog nerve muscle preparations by electrical stimulation with metal wires [25]. From that study, tools for analyzing a cell's electrical properties have development over the years. Conventional patch clamp and probing were have been the classical platform tools for characterization of single cell electrical properties.

\subsection{Conventional Patch Clamp}

The patch clamp technique is unique in enabling high-resolution recording of the ionic currents flowing through a cell's plasma membrane. Since the introduction of the patch-clamp technique by Neher and Sakmann in 1976, patch-clamp was adopted by researchers in cellular and molecular biology research areas for studying and providing valuable information of biological cell electrical properties [26,27]. The patch-clamp technique is also capable to analyze ionic currents in the cell membrane under conditions of complete control over transmembrane voltage and ionic gradients. Figure 1a illustrates the basic principle of patch clamp technique. A glass micropipette is used as a probe to suck a cell membrane into a micropipette to form a high electrical resistance or also called as giga-seals (e.g., normally between 10 and $100 \mathrm{G} \Omega$ [28]. Thus, the ion current that flows through the pipette (containing an electrode) is measured through an amplifier. Patch clamp can be operated in two modes, which are voltage and current mode. Voltage mode is used to measure voltage specific 
activity of an ionic channel, while current mode is used to measure the potential change in membrane when a current pulse has been injected into the cell [29]. Furthermore, the patch clamp technique has five basic measurement configurations such as cell-attached patch (CAP), whole-cell (WC), inside-out patch (IOP), outside-out patch (OOP) and permeabilized-patch WC-configuration (ppWC) [30,31]. More detail on the working principle of the patch clamp technique has been described $[32,33]$.

The work of Hamill et al. sparked an approach for obtaining information about the characteristics and distribution of ion channels in living cells [28]. They used frog muscle fibres and rat myoballs as cell samples to detail several variants of this technique to create complete electrical isolation of the patched membrane for a variety of cells. This whole cell configuration is the most often utilized mode of the patch clamp technique. Zhang et al. combined the whole-cell patch clamp with fluorescence ratio imaging for measuring the electric properties of a cell membrane [34]. Fluorescence dye was used to monitor the transmembrane potential change of the cell in the long term without seriously perturbing the intracellular milieu. Both techniques combined have been successfully used to distinguish between differentiated and undifferentiated N1E-115 neuroblastoma cells according to the values of the resting potentials.

The conventional patch clamp technique has several disadvantages. First, the patch clamp technique is time consuming process $[29,35]$. The entire dish of cells needs to be replaced after the extracellular fluid has been manipulated, before continue the recording. Second, the quality of the cell and suspension must remain in good condition for channel expression to be homogenous [29]. Third, an experienced operator is required to move the glass pipette over the single cell for measuring current and voltage changes across the membrane through ion channels without damaging the whole cell. Other issues arise such as recoding quality and temperature control. Nevertheless, the patch clamp technique offers high sensitivity (pA resolution) and allows low noise measurement of the currents passing through the low conductance $(\mathrm{pS})$ ion channels [25]. The evolution of upgraded modifications of the patch clamp technique can be found elsewhere [36].

\subsection{Nanoprobe}

Nanoprobes could potentially be used to perform single cell's electrical characterization. The nanoprobe capable to measure direct electrical properties of single cell and quantitatively determine the viability of single cells. M. R. Ahmad et al. developed a dual nanoprobe integrated with nanomanipulator units inside environmental scanning electron microscope (ESEM) to perform electrical probing on single cells for novel single cell viability detection [37]. Figure 1b illustrated the working principle of dual nanoprobe for single cell electrical measurement. Based on Ohm's law, current flow passing through the intracellular area of the cell was measured when a dual nanoprobe penetrated the intracellular area. ESEM was used for high resolution observation while preserving the cell's native state even when the cell is moving out of its buffer [38]. This technique successfully differentiated the live and dead cells of W303 wild yeast cells based on the electrical properties of the cell [37]. Recently, electrostatic force microscopy (EFM) was utilized to quantify the electric polarization response of single bacterial cells with high accuracy and reproducibility [39]. They demonstrated effective dielectric constants obtained from the different bacterial types (Salmonella 
typhimurium, Escherichia coli, Lactobacillus sakei and Listeria innocua), which were well correlated with the hydration state of bacteria. Figure 1c illustrated the working procedure of effective dielectric constant measurement using EFM. The electric polarization force between a bacterium and a nanometric-conducting tip mounted on a force-sensing cantilever was measured at different positions. Topographic images were used to obtain the geometry of the bacterium and finite element numerical simulations of a homogeneous bacterium were utilized to measure the effective dielectric constants of the cell $[39,40]$. A simple single cell electrical model was used in order to measure electrical properties of yeast cells. However, this technique requires a skilled operator to perform the measurement and is time consuming. The device is bulky system, which can only be performed in a restricted area, e.g., clean room [40].

\section{Microfluidics Platforms}

An advance in microfabrication technique, such as soft lithography, creates new opportunities for producing structures at micrometer scale inexpensively and rapidly [41]. For this reason, we have witnessed rapid development of microfluidics system for more than a decade ago for biology and medical research [1,14,42]. Microfluidics systems are a science and technology of manipulating fluids at the submillimetre length scale in the microscale fluidic channel. Microfluidics recognized as micro total analysis systems ( $\mu \mathrm{TASs}$ ) [43] or lab-on-a-chip (LoC) technologies have attracted attention because of the potential to improve diagnostics and biology research. Microfluidic systems have shown a potential to become widely adopted in modern clinical diagnosis and biology research (e.g., DNA analysis [44] and cell analysis [45]) because they are reproducible, have low power consumption, less sample and reagent consumption, are economical, amenable to modifications and can be integrated with other technologies $[46,47]$. The ability of microfluidics system to perform early cancer detection and address some problems in cellular analysis, make them suitable to replace the classical technique in single cell electrical analysis. Several microfluidic systems have been developed for single cell electrical properties analysis, such as electrorotation, impedance flow cytometry and microelectrical impedance spectroscopy ( $\mu$ EIS).

\subsection{Electrorotation}

A cell shows a rotated ability when it is placed into a rotating electric field within a medium with a non-uniform electric field. Analysis of these phenomena called an electrorotation (ROT), is commonly used for measuring the dielectric properties of cells without invasion. ROT measurement theory is based on rotational speed of cells/particles when the cell and the suspending medium have different electric polarizability, by referring to the frequency of a rotational electric field. This electric field is generated by quadrupole (arranged in a crisscross pattern) electrodes and each electrode is connected to an AC signal with a $90^{\circ}$ phase difference from each other. 


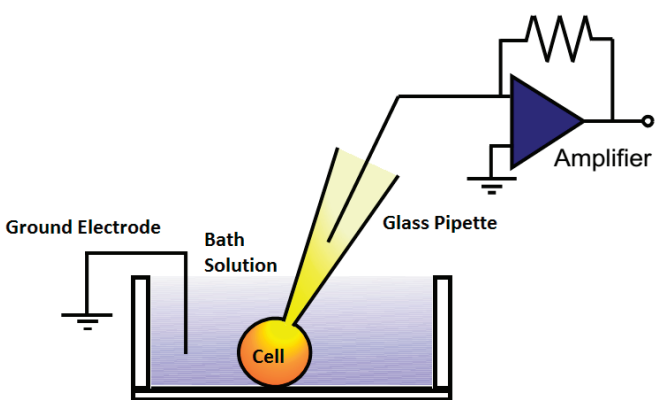

(a)

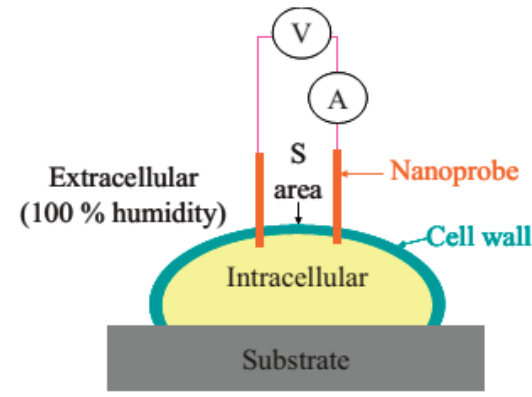

(b)

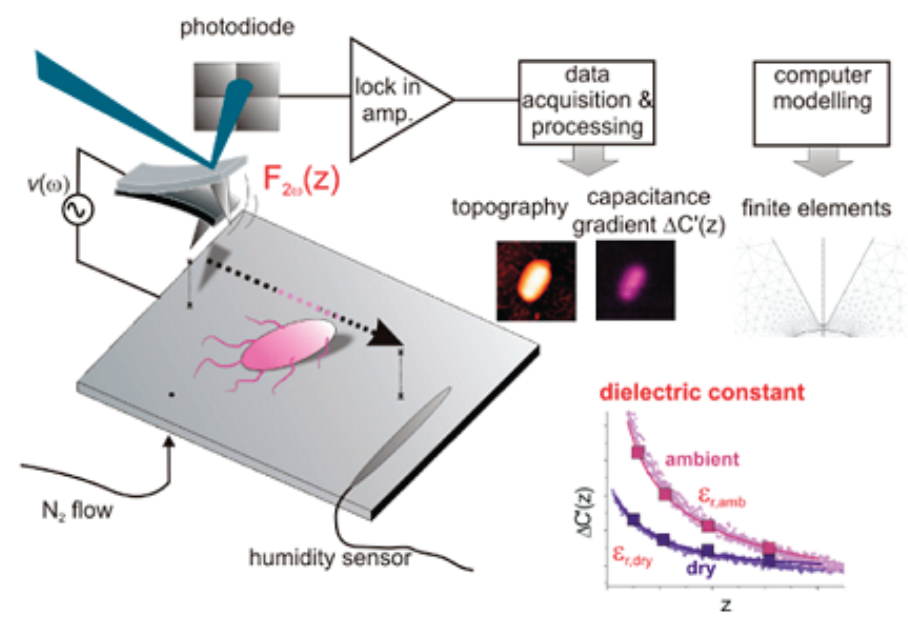

(c)

Figure 1. (a) Schematic diagram of conventional patch clamp technique; (b) Single cell electrical measurement using dual nanoprobes incorporated with ESEM. Reprinted with permission from [37]; (c) Schematic of measurement of the effective dielectric constant of a single bacterium using electrostatic force microscopy. Reprinted with permission from [39].

The quadrupole electrodes connected to sine wave was a famous design in ROT technique [48-50]. Figure 2a shows a working principle of ROT, four electrodes were energized by sinusoidal signal generator created rotating electrical field, E. Laser tweezers were used to drag a single cell to the center of a four-electrode chamber, then a single cell, $\mathrm{P}$ will rotate in either the same direction (co-field) or in the opposite direction (anti-field) to the rotating field [51]. The direction was taken by the cell depending to the dielectric properties of the cell and suspending medium along with the frequency of the electric field. The dielectric properties of a single cell can then be extracted by utilizing Maxwell's mixture theory, to associate the complex permittivity of the suspension to the complex permittivity of the cell [49]. More detail on theory and working principle of electrorotation can be found in other articles [52-54] and a book [55]. 


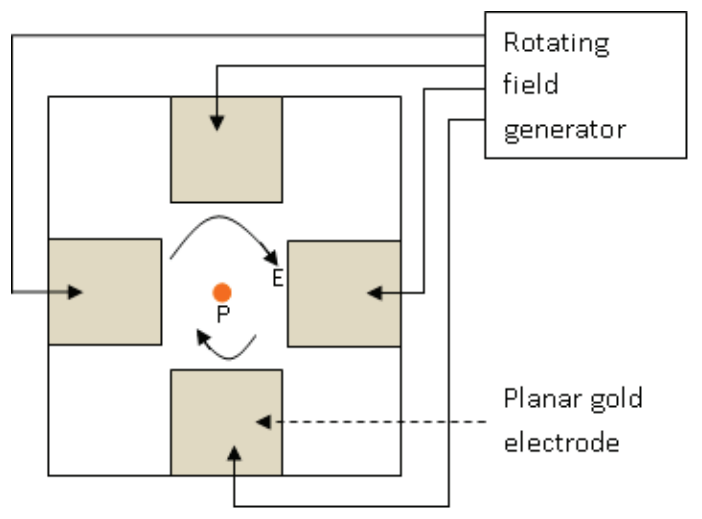

(a)

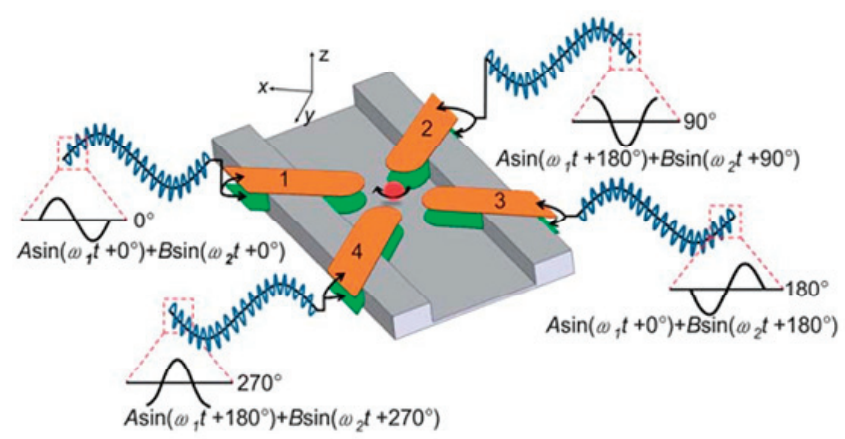

(b)

Figure 2. (a) An illustration of the working principle of electrorotation to analyse single cells; (b) The electrorotation (ROT)-microchip incorporated with the 3D octode. nQDEP (negative quadrupole dielectrophoresis) signal, Asin $\left(\omega_{1} t+0^{\circ}\right)$ and Asin $\left(\omega_{1} t+180^{\circ}\right)$ are used for a single cell trapping, while the ROT signals, Bsin $\left(\omega_{2} t+0^{\circ}\right)$, Bsin $\left(\omega_{2} t+90^{\circ}\right), B \sin \left(\omega_{2} t+180^{\circ}\right)$ and Bsin $\left(\omega_{2} t+270^{\circ}\right)$ are used to simultaneously generate torque. Reprinted with permission from [56].

In the ROT technique, the amplitude of the electric field remains unchanged because the cells are only rotated at a certain position in an electric field [57]. Therefore, it is suitable for fitting the rotation spectra at frequency range from $1 \mathrm{kHz}$ to around $200 \mathrm{MHz}$ to determine the intrinsic electrical properties of single cells such as cytoplasm conductivity, cytoplasm permittivity and specific membrane capacitance $[48,58,59]$. Electrorotation spectra are referred to cellular rotation rate versus frequency of the applied field. Jun Yang et al. [48] used frequency range between $1 \mathrm{kHz}$ and $120 \mathrm{MHz}$, to fitting the rotation spectra in order to extract dielectric properties (membrane capacitance) of four main leukocyte subpopulations, i.e., T- and B-lymphocytes, monocytes, and granulocytes. From this experiment, ROT was capable to characterize the dielectric properties of cell subpopulations within a cell mixture. In addition, ROT was utilized to determine the cell viability at real time assessment $[51,60,61]$. C. Dalton demonstrated that electrorotation technique can be used to determine the viability of two intestinal parasites, i.e., Giardia Intestinalis and Cyclospora 
Cayetanensis [51]. An ellipsoidal two-shell model [58] was utilized to analyse the data and estimate the electrical parameter value.

Recently, the concept of negative quadrupole dielectrophoresis (nQDEP) and ROT signals superposed on each other in electrorotation technique was reported (Figure 2b). An accurate ROT spectrum was measured without any other disturbances because repulsive force by the nQDEP signal is stronger [56]. Specific membrane capacitance and cytoplasm conductivity of human leukocyte subpopulations (T-lymphocytes, B-lymphocytes, granulocytes, and monocytes) and metastatic cancer cell lines ( $\mathrm{SkBr} 3$ and $\mathrm{A} 549)$ were well achieved. Although the electrorotation technique is powerful tool, capable of extracting the electrical properties of the cell, such as cytoplasm conductivity and membrane permittivity, ROT technique has several drawbacks. Time consumption is a major factor for why the ROT technique has been unable to enter the modern clinical disease diagnosis as an analysis tool. G. De Gasperis et al. and M. Cristofanilli et al., utilized ROT technique to analyze single cells and it took approximately $30 \mathrm{~min}$ to test a single cell $[62,63]$. These reports indicate that electrorotaion is a slow technique. Electrorotation also requires a skilled operator to position a single cell in the middle of a rotating electric field and also count the number of revolutions made by particles [64]. Nevertheless, electrorotation is a noninvasive technique which allows it to be used in sequential investigations. ROT also operates at a single-organism level and does not require extensive cell preparations [59]. Table 1 shows a summary of a microfluidic device using electrorotation technique for single cell electrical characterization.

\subsection{Impedance Flow Cytometry}

Flow cytometry is a fundamental and powerful analytical tool in cell biology and cellular disease diagnosis for many years. Flow cytometry has an ability to address some problems in single cell analysis such as identifiying, counting and sorting cells $[68,69]$. Based on laser-induced fluorescence detection in flow cytometry for single-cell studies within cell populations of relatively large sizes [70], flow cytometry creates an ideal scenario to analyze single cell electrical properties from a cell population. Coulter [71] developed the first flow cytometry tool having capability to measure the electrical properties of single particles, which is known as the microfluidic Coulter counter. A Coulter counter measures the changing of DC resistance between two electrically isolated fluid-filled chambers when microparticles act as an insulating layer at DC pass through a small connecting orifice. Figure 3 illustrates the working principle of the Coulter counter, where two large electrodes are placed on connecting chamber. When a particles or biological cells flow through a sensing aperture which has current flow, it will displace the conductive fluid and alters the resistance. The current flow was decreased as a particle passes through and for this reason, individual cells can be counted and sized [72]. The Coulter counter is limited to counting cells and classifying cell types based on size due to challenging of selecting electrode design and channel geometry [73]. The Microfluidic Coulter counter is incapable of characterizing electrical properties of cell. In order to determine the electrical properties of cells, Sohn et al. [74] developed flow cytometry based on capacitance principle to measure the DNA content of fixed eukaryotic cells. Electrical properties of individual cells were referred to distinct peaks measured by a capacitance bridge at $1 \mathrm{kHz}$ frequency. 


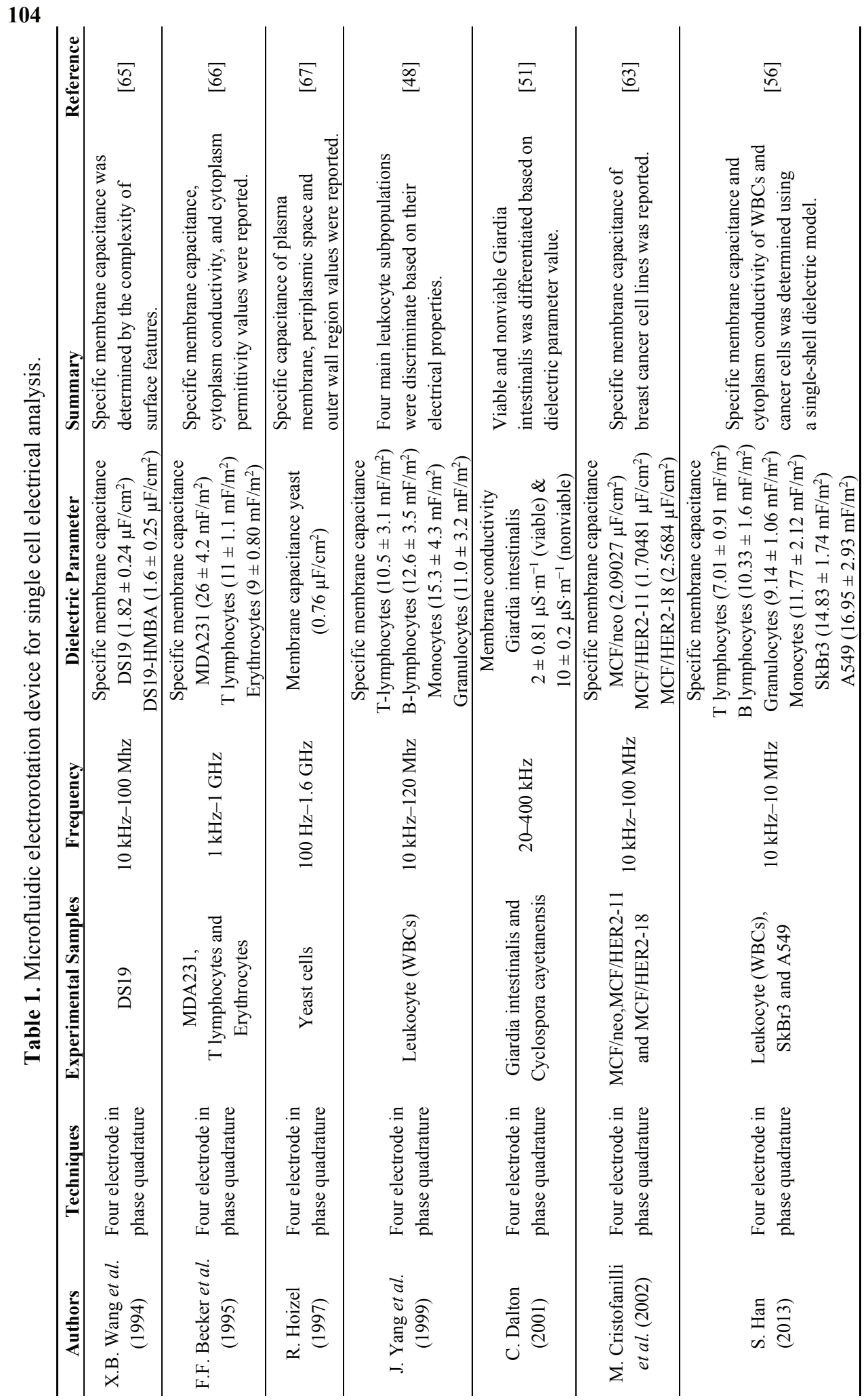




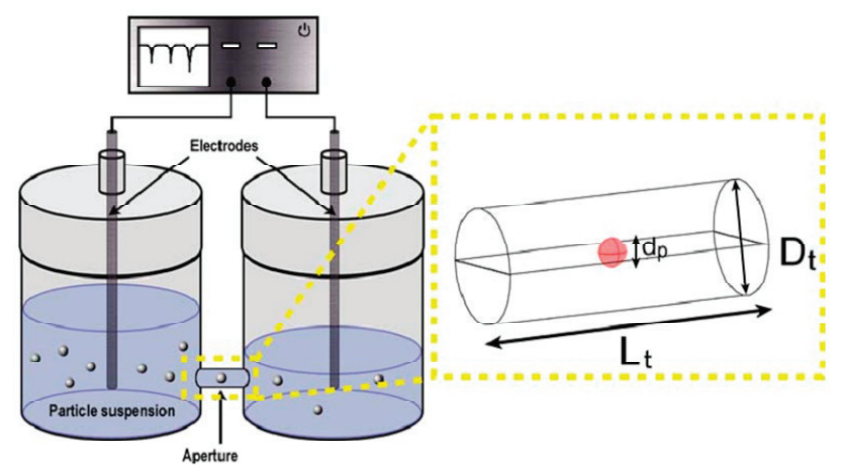

Figure 3. Schematic diagram of the Coulter counter working principle. Reprinted with permission from [72].

Gawad et al. [75] developed a significant device in single cell impedance technology, which is known as the impedance flow cytometry (IPC). This device used coplanar electrodes to measure clear differentiation of beads and also erythrocytes and ghost cells (ghosts are RBCs that have been lysed in hypotonic buffer, leaving behind a membrane sack filled with ionic solution). As shown in Figure $4 \mathrm{a}$, three microelectrodes were fabricated on the bottom of a microfluidic channel. An AC voltage was supplied to energise the electrodes for generating a non-uniform electric field within the channel. The impedance value within channel was changed, when a single cell was flowing through the detection area. This impedance value was used to characterize the electrical properties of single cell. However, this electrode configuration may affect impedance measurement when single cell was at variation position. To address this issue, K. Cheung et al. [76] designed parallel facing electrodes in a microfluidic channel (Figure $4 b$ ). One pair of parallel electrodes was used to detect cells and measure electric current fluctuation, whereas the other one was acted as a reference. Then, the difference between the two signals was measured. The device has the ability to measure electrical properties of normal RBCs and glutaraldehyde-fixed RBCs. More details for the derivation of the electric field distribution for two different electrode configurations, based on Schwarz-Christoffel Mapping (SCM) have been described [77]. In addition, a similar system (parallel facing electrodes) was used by Kampmann et al. [78] to monitor frequency effect during conducted measurement processes. The result showed that the cell can be accurately sized at around $500 \mathrm{kHz}$, where low frequency behaviour is dominated by the electrical double layer (EDL). Meanwhile, at intermediate frequencies behaviour is dominated by the membrane capacitance and at high frequencies, the cell cytoplasm becomes important. High frequency $(8.7 \mathrm{Mhz})$ measurements were used to detect infection of RBCs with the parasite Babesia bovis based on the changes in the electrical properties of the cell cytoplasm [79]. Recently, an impedance flow cytometry that covers frequency range from DC up to $500 \mathrm{Mhz}$ was developed by Niels Haandbæk et al. [80]. The device has a capability of dielectric characterization of subcellular components of yeast cells, such as vacuoles and cell nuclei, and can be used for discriminating wild-type yeast from a mutant. 


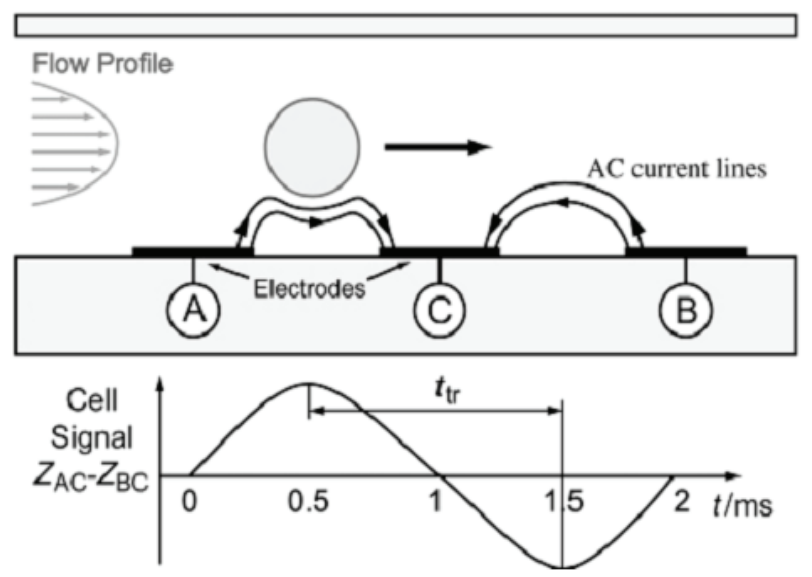

(a)
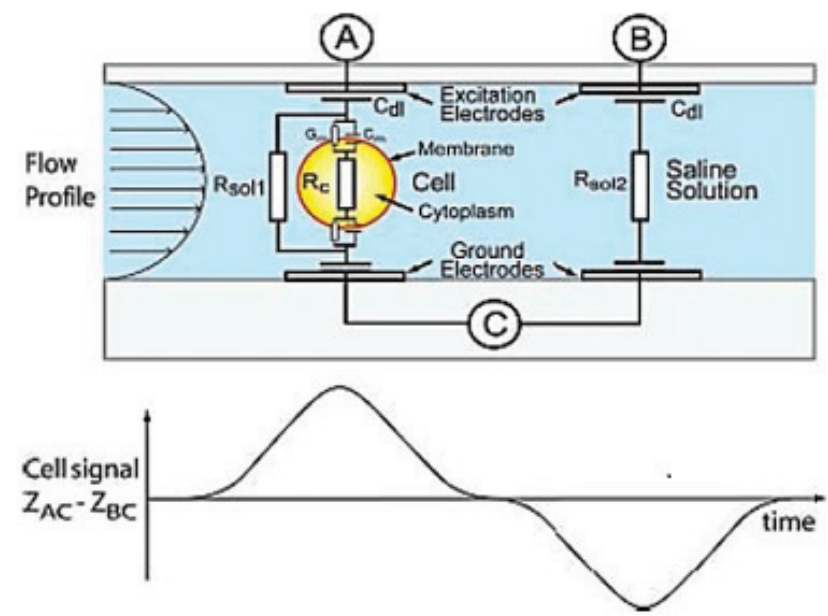

(b)

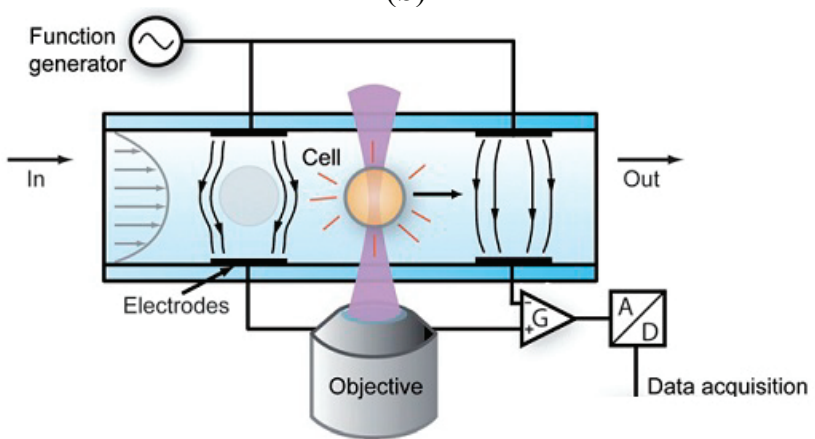

(c)

Figure 4. Cont. 


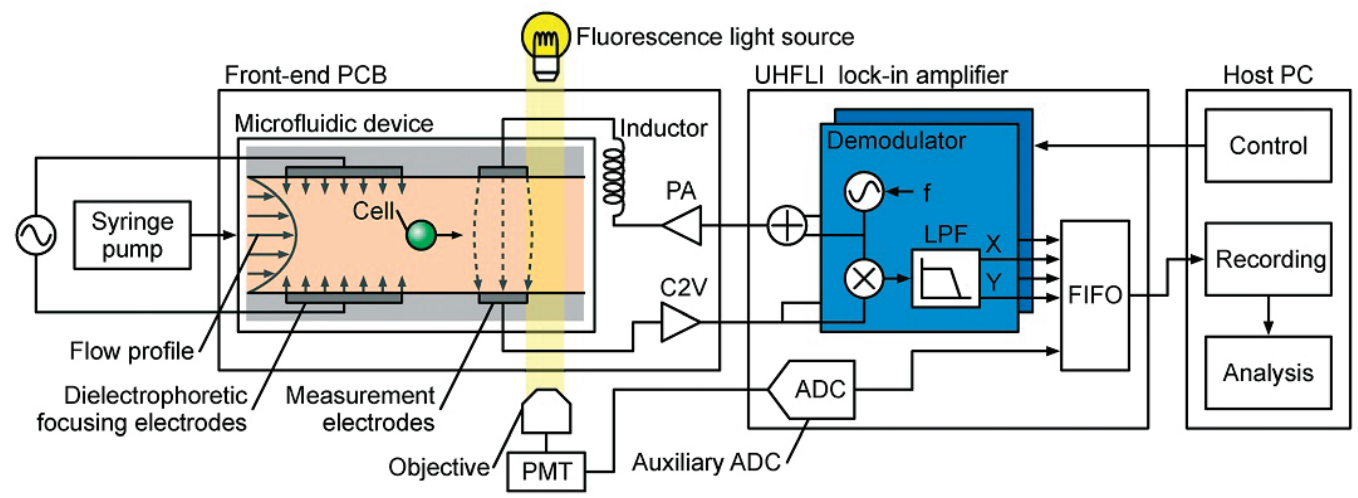

(d)

Figure 4. (a) Illustration of a particle flowing over three electrodes inside a microfluidic channel, and a typical impedance signal for a single particle. Reprinted with permission from [75]; (b) A single cell flowing over one pair of electrode and second pair used as reference is shown. Reprinted with permission from [76]; (c) Schematic diagram of the micro impedance cytometer system, including the confocal-optical detection. Reprinted with permission from [81]; (d) Schematic of the complete microfluidic cytometer. The lock-in amplifier drives the series resonance circuit, formed by the discrete inductor and the impedance between the measurement electrodes, with an alternating current (AC) signal at a frequency close to resonance. Reprinted with permission from [82].

Holmes et al. [81] demonstrated measurement and differential of single cells at a high speed level by using microfluidic flow cytometry with an attached fluorescence measurement unit (Figure 4c). The device accurately identified T-lymphocytes, monocytes and neutrophils of WBC and a full three-part differential count of whole human blood was achieved. Despite single cells being measured, the data represented the average of the population and was not accurately measured on an individual cell. In addition, similar research groups developed an integrated microfluidic impedance flow cytometry system with haemoglobin concentration measurement unit [83] and RBC lysis [84]. In order to increase the signal-to-noise ratio relative to a single-phase, unfocused stream, while to avoid large shear forces on cells, Mikael Evander [85] developed a microfluidic impedance cytometer that utilizes dielectrophoretic focusing technique. This technique was used to center cells in a fluid stream, thus forms the core of a two-phase flow. Then, this flow will pass between electrodes for analysis of cells at various frequencies from range $280 \mathrm{kHz}$ to $4 \mathrm{MHz}$. As a result, this technique is able to distinguish between red blood cells and platelets and between resting and activated platelets.

A label-free cell cytometry based on electrophysiological response to stimulus was reported [86]. This method recorded a cell's functionality rather than its expression profile or physical characteristics. In order to distinguish different cells types, they used nature electrically excitable cells that are activated by sufficient transmembrane electric fields. During this activation, the extracellular field potential (FP) signal from cells was produced and detected by electrode inside 
microchannel. Human induced pluripotent stem cell-derived cardiomyocyte (iPSC-CM) clusters from undifferentiated iPSC clusters were differentiated by using these signals. A contactless measurement method to perform single cell impedance cytometry using a disposable biochip integrated with a printed circuit board that has reusable electrodes was reported [87]. The device can detect and measure impedance of biological cells in a real biological sample (e.g., whole blood (sheep)) and also significantly reduces the manufacturing costs. Recently, a microfluidic impedance cytometer, incorporated with an electrical resonator was reported. This device is high sensitive and capable to measure at high frequencies. Figure $4 \mathrm{~d}$ showed microfluidic system integrated with a resonant circuit which consists of a discrete inductor in series with the impedance between the measurement electrodes [82]. The cells detection principle is based on the resonance- enhanced phase shift of the measurement current induced by cells or particles passing through the microfluidic channel. Discrimination based on the differences in dielectric properties of E. coli and B. subtilis was well achieved. T. Sun et al. extends impedance measurements from one dimension to two or three dimensions by utilized electrical impedance tomography (EIT) [88]. A circular 16-electrode array with equal spacing was fabricated and images of Physarum polycephalum were reconstructed by measuring the voltages across sequential electrode-pair combinations. Human fibroblast cells were used to differentiate between an environment of growth medium with and without cells using EIT [89]. Table 2 shows a summary of microfluidic impedance flow cyometry techniques for single cell electrical characterization.

\subsection{Micro Electrical Impedance Spectroscopy ( $\mu$-EIS)}

Micro electrical impedance spectroscopy ( $\mu$-EIS) is a technique where dielectric properties in a frequency domain of a cell is measured to characterize and differentiate the various types of cell. Mainly this technique analyzed the current response when a single cell was trapped in a trapping system where an alternating current (AC) was applied across the trapping zone. A trapping system is a major contribution and significant part in $\mu$-EIS device. For this reason, development of a trapping system is very crucial and varieties of the trapping system have been developed, such as hydrodynamic traps, negative pressure traps and DEP traps.

First development of micro electrical impedance spectroscopy ( $\mu$-EIS) was reported in 2006 [97]. They developed microfluidic device which utilized the negative pressure to capture the single cell into the analysis cavity (Figure 5a). This device was used to measure the electrical impedance of human breast cancer cell lines of different pathological stages (MCF-7, MDA-MB-231, and MDA-MB-435) [18]. However this device has a disadvantage to monitor the cell capturing process using a microscope because the contrast difference in the silicon nitride membrane composing the cell traps area and the surroundings. The same group, Cho et al. [4] developed an array of horizontal cell traps of an $\mu$-EIS device to overcome the limitation of the previous device. Negative pressure was used to capture single cells and impedance measurement was performed to obtain the electrical impedance spectra of metastatic head and neck cancer (HNC) cell lines. This device also can minimize the leakage current due to the position of cells formed in direct contact between cells and electrodes. 


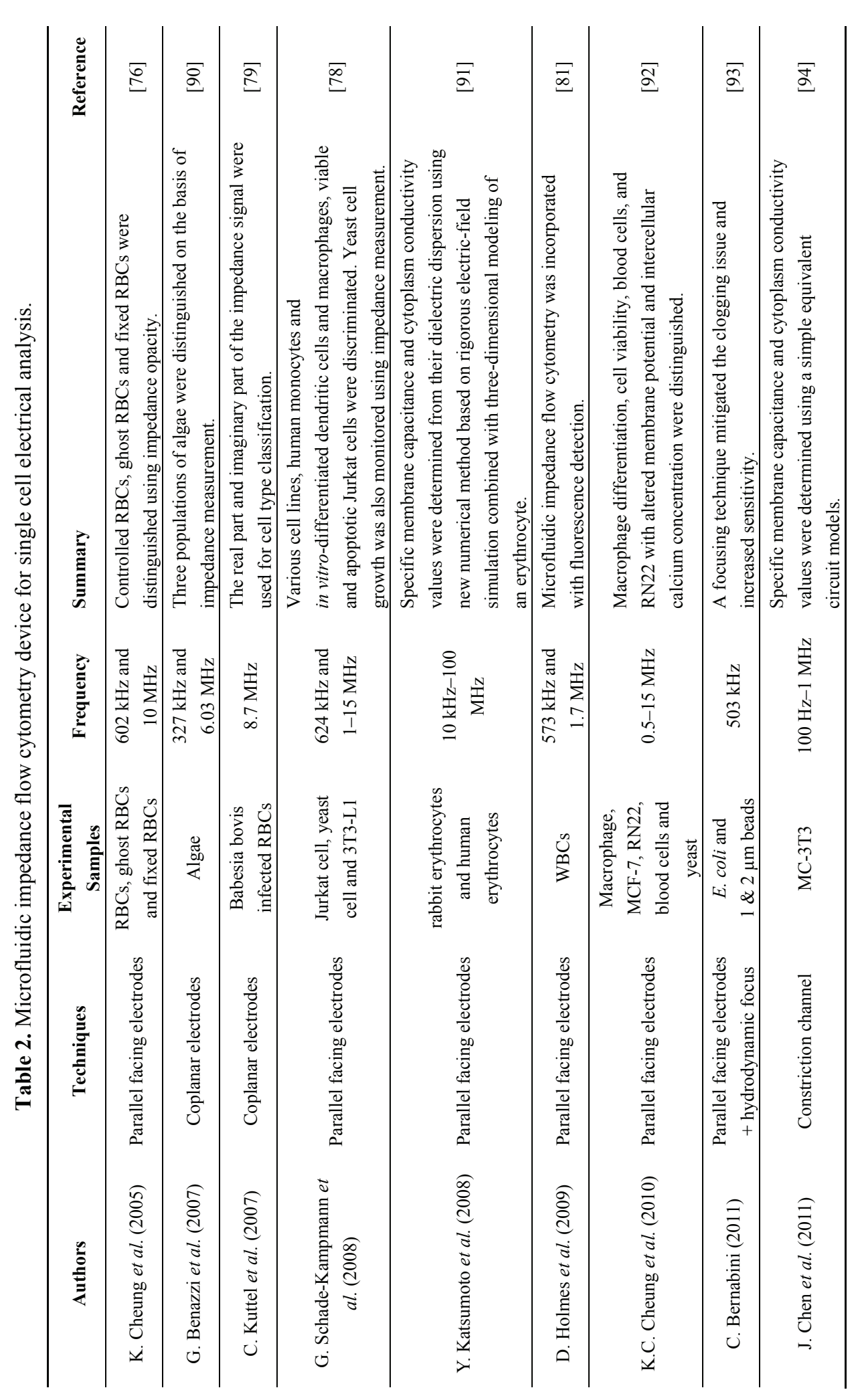




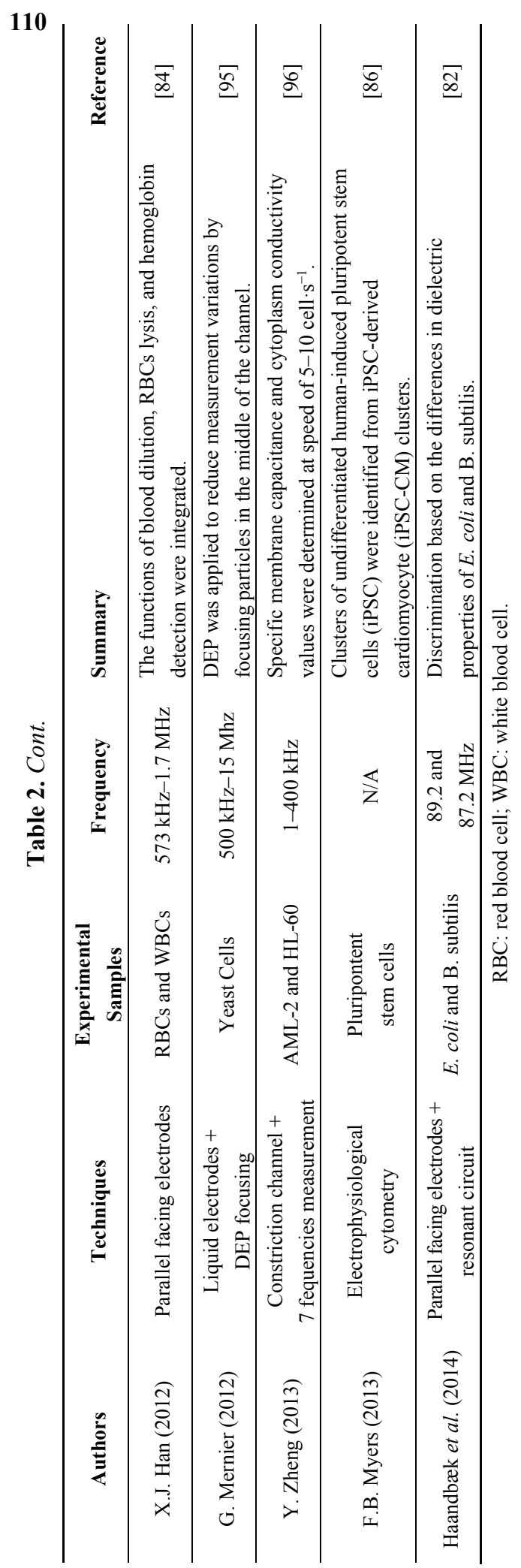


Furthermore, the concept of vertical trapping system in $\mu$-EIS has been used to monitor the dynamic change of single cell electrical properties over a period of time [98,99]. Hydrodynamic trapping system (e.g., micropillars) within a microfluidic channel was developed by Jang et al. [21]. Figure $5 \mathrm{~b}$ showed the micropillars structure inside microfluidic channel and capable to capture physically single cells. A single human cervical epithelioid carcinoma (HeLa) was successfully captured by the micro pillars and its impedance was measured. Mondal et al. performed impedance measurement of HeLa cell based on two geometry structures of micropillars trapping system, namely, parallel and elliptical geometry [100]. Malleo et al. demonstrated a hydrodynamic trapping device which has a differential electrode arrangement that measures multiple signals from multiple trapping sites. Measurements was performed by recording the current from two electrode pairs, one empty (reference) and one containing HeLa cells [101]. The device continuously monitored the toxin activity at the single cell level.

Recently, the concept of dielectrophoresis (DEP) for trapping system was reported [102]. The non-uniform electric field distribution between the top and bottom electrodes caused the red blood cells (RBCs) to experience positive dielectrophoresis at $80 \mathrm{kHz}$ frequency [103]. As a result, the red blood cells have been trapped inside microwells, thus the impedance of RBCs was measured [102]. Another DEP trapping technique was developed by Tsai et al. to capture a single HeLa cell, then impedance measurement was performed [104]. Figure 5c illustrated trapping system using DEP [104]. Despite that microelectrical impedance spectroscopy ( $\mu$-EIS) has several advantages such as label free, real time measurement and non invasive, $\mu$-EIS also has some drawbacks. For example, $\mu$-EIS requires theoretical model for data analysis [105] and time consuming (trapping and releasing process take time to be completed) [106]. Table 3 shows a summary of microelectrical impedance spectroscopy technologies for analyzing the single cell's electrical properties.

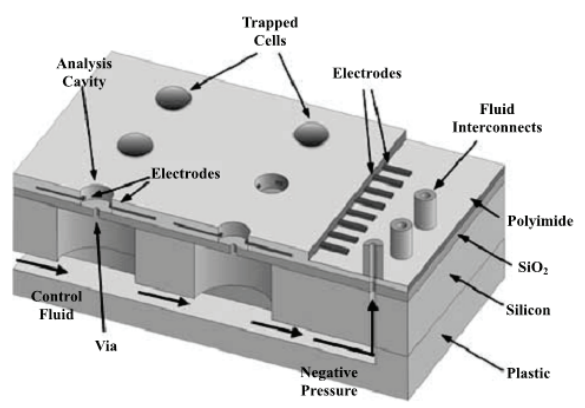

(a)

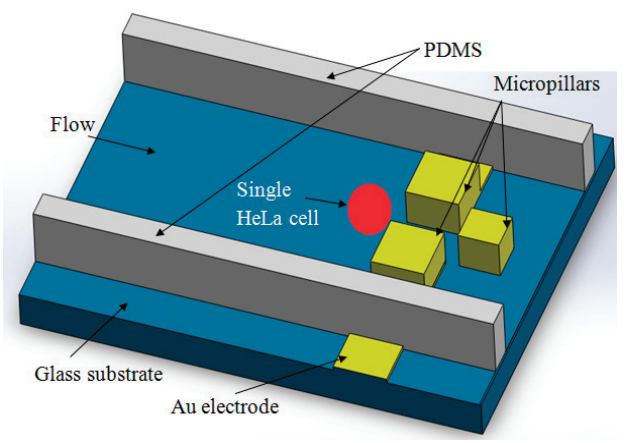

(b)

Figure 5. Cont. 


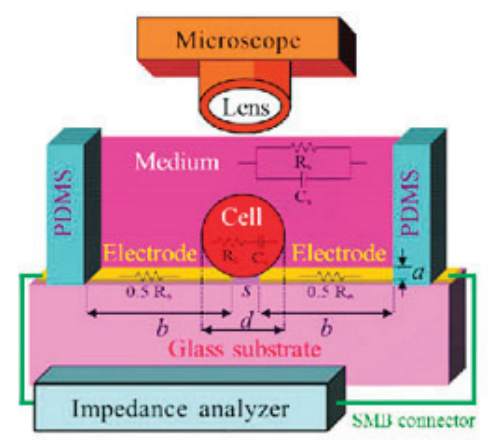

(c)

Figure 5. (a) Illustrated a micro electrical impedance spectroscopy system using multielectrode configurations within an analysis cavity. Reprinted with permission from [97]; (b) Shown 3D schematic of the $\mu$-EIS device incorporated with micropillars structure for capture the single cells; (c) Schematic diagram of cell measurement using DEP cell trapping technique. Reprinted with permission from [104]. 


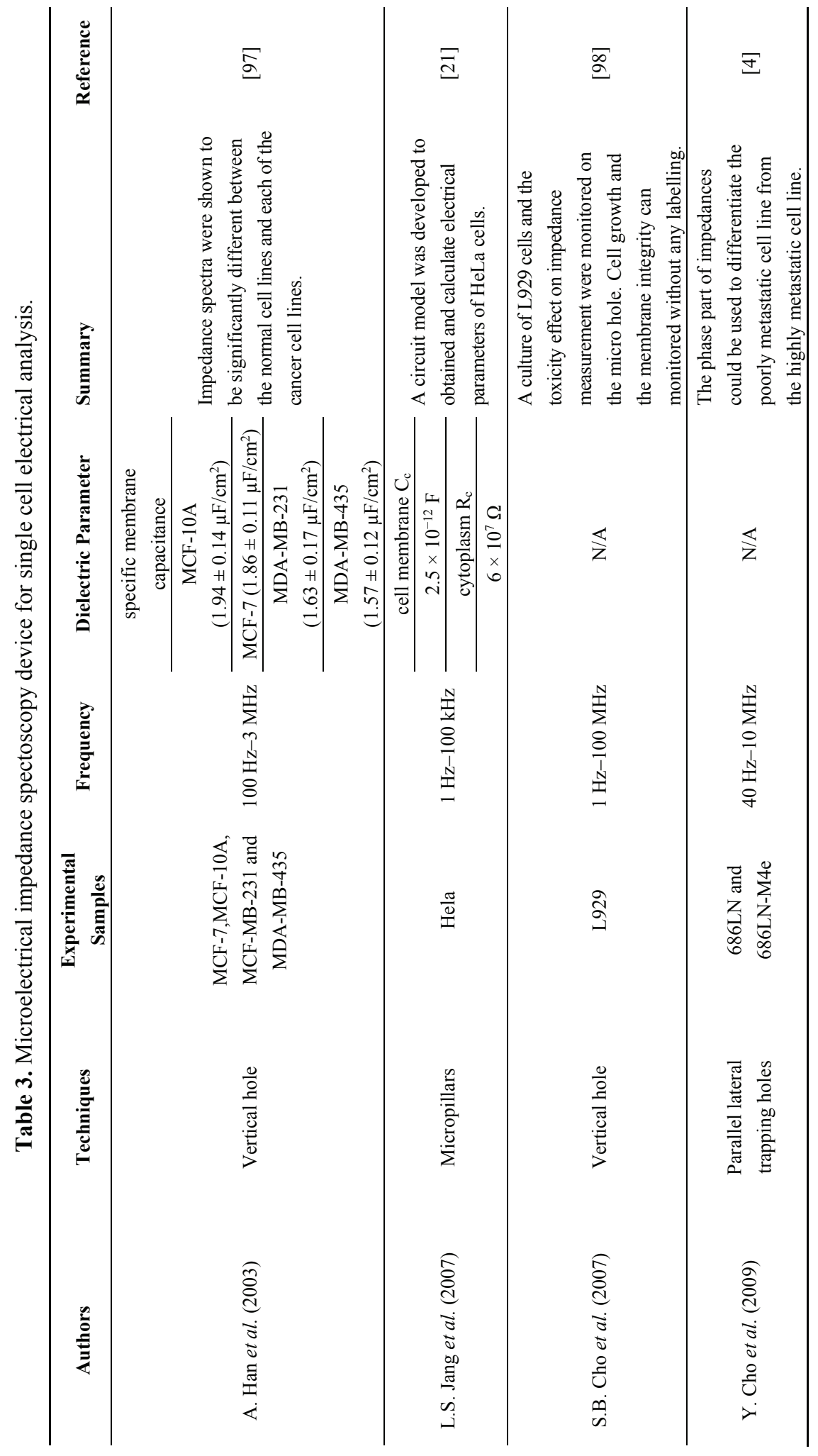

113 


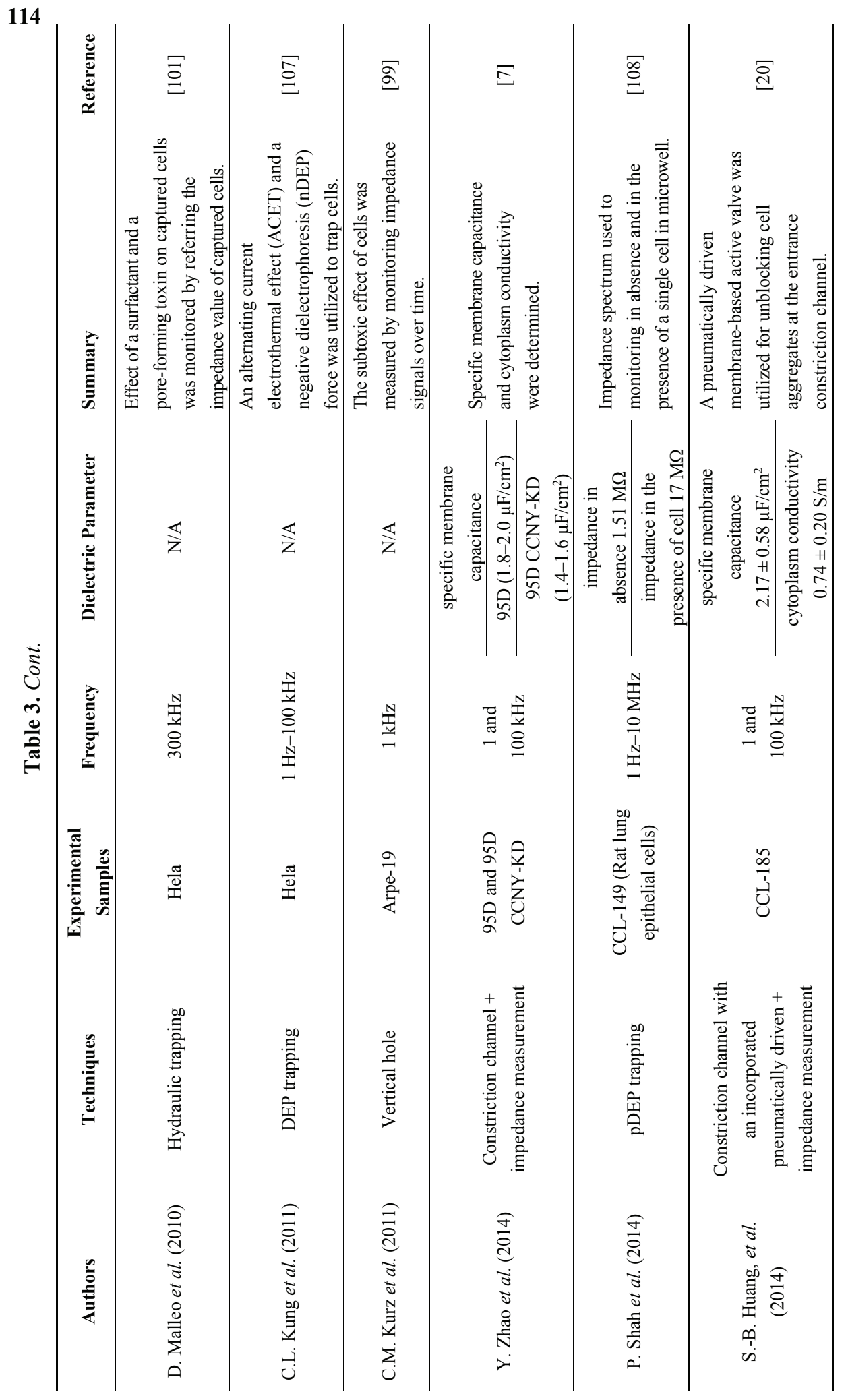




\section{Discussion}

The rapid development of single cell analysis tools (e.g., biophysical and chemical characterizations) can be seen based on the hundreds of review and technical papers currently published every year [109]. Clearly, the growing interest in this research field demonstrates its practical value from the viewpoint of proof of concept and applications. The traditional platform is a basic foundation to provide the most straight-forward mechanisms to analyze electrical properties of individual cell. However this approach suffers from low throughput, delicate protocol, requires an experienced operator and bulky experimental set-up [110]. For instance, in clinical application, high throughput devices are significantly required to test a large number of cells (e.g., blood) in order to obtain low numbers of meaningful data (e.g., CTC cells) [57]. Nevertheless, traditional platforms have provided fundamental insights to the microfluidic development. The microfuidics device is a promising technique to understand the cellular heterogeneity and overcome the limitation of traditional technique. For that reason, three microfluidics techniques (electrorotation, impedance flow cytometry and microelectrical impedance spectroscopy) have been developed to analyze and characterize the single cell's electrical properties. Among these techniques, microfluidic impedance flow cytometry (IFC) is a technique used widely in clinical diagnosis because of high throughput during count and differentiation of the WBCs. For example, parallel facing electrodes device achieved $\sim 100$ cells $\cdot \mathrm{s}^{-1}$ and is capable of testing a large number of cells for obtain statistically meaningful data [81]. Microfluidic impedance flow cytometry has been demonstrated to distinguish various single cells (16 types of cell) based on the electrical properties conditions. Meanwhile, 13 types of cell were distinguished by electrorotation and microelectrical impedance spectroscopy.

Electrical measurements can also be incorporated with a cell sorting unit to collect cells having different physical properties for further biochemical assaying. AC dielectrophoretic (DEP) for sorting live cells from interfering particles of similar sizes by their polarizabilities under continuous flow was reported [111]. DEP forces induced by the AgPDMS electrodes were used to manipulate cells to move toward high or low electric field regions, depending on the relative polarizability between the cells and their suspending medium. Jun Yang et al. utilized magnetically activated cell sorting (MACS) for obtaining the subpopulations from human peripheral blood (B-lymphocytes and monocytes), thus performing the single cell electrical properties measurement by electrorotation techniques [48].

Microfluidic devices have demonstrated great potential in realizing electrical measurements on single cells at a higher testing speed and label free approach. Electrical measurements on single cells can be used to indicate possible diseases and it suitable for disease prescreening application. From prescreening processes, future examinations can be done to evaluate the disease condition. Table 4 shows a summary of comparisons between three microfluidic methods. The microfluidic techniques were discussed have some potential applications in biological and medical application $[18,44,92,101,112]$. 
Table 4. Comparisons between three microfluidic techniques.

\begin{tabular}{clll}
\hline $\begin{array}{c}\text { Approaches } \\
\text { Technique }\end{array}$ & Advantages & Disadvantages & Applications \\
\hline Electrorotation & $\begin{array}{l}\text { Capable to quantifying } \\
\text { a cell's intrinsic } \\
\text { electrical properties }\end{array}$ & $\begin{array}{l}\text { Low throughput and } \\
\text { limitation to low conductivity } \\
\text { sucrose buffer solution }\end{array}$ & Monitor parasite; Cell separation \\
\hline $\begin{array}{c}\text { Impedance flow } \\
\text { cytometry }\end{array}$ & High throughput & low specificity & $\begin{array}{l}\text { Cell sorting and counting; } \\
\text { Cell impedance variations; } \\
\text { DNA hybridization detection }\end{array}$ \\
\hline $\begin{array}{c}\text { Microelectrical } \\
\text { impedance } \\
\text { spectroscopy }\end{array}$ & $\begin{array}{l}\text { Characterizing ion } \\
\text { channel activity }\end{array}$ & $\begin{array}{l}\text { Low throughput and } \\
\text { size-independent parameters }\end{array}$ & $\begin{array}{l}\text { Cancerous stage screening; } \\
\text { Toxin detection }\end{array}$ \\
\hline
\end{tabular}

\section{Conclusions}

The presented review of selected research works on single cell electrical properties provides information on technological development in single cell electrical characterization from traditional approaches to current microfluidic approaches. Microfluidics technology opens a new paradigm in cellular and microbiology research for early disease detection and provides critical information needed by research scientists and clinicians for improved clinical diagnosis and patient outcome. The recent excellent achievements in microfabrication techniques have enabled the rapid development of microfluidic technologies for further practical applications for the benefit of mankind. Furthermore, microfluidic technological progress has provided additional advantages such as reduced complexity of experiment handling, lower voltage on the electrodes, faster heat dissipation, small volume of reagents used, and in situ observation of the cell response [113].

\section{Acknowledgments}

This work was supported by grants from the Ministry of Education Malaysia (grant No. 4L640 and 4F351) and Universiti Teknologi Malaysia (grant No. 02G46, 03H82 and 03H80).

\section{Author Contributions}

Muhammad Asraf Mansor and Mohd Ridzuan Ahmad wrote and edited the review.

\section{Conflicts of Interest}

The authors declare no conflict of interest.

\section{References}

1. El-Ali, J.; Sorger, P.K.; Jensen, K.F. Cells on Chips. Nature 2006, 442, 403-411.

2. Suresh, S. Biomechanics and Biophysics of Cancer Cells. Acta Biomater. 2007, 3, 413-438. 
3. Coley, H.M.; Labeed, F.H.; Thomas, H.; Hughes, M.P. Biophysical characterization of MDR breast cancer cell lines reveals the cytoplasm is critical in determining drug sensitivity. Biochim. Biophys. Acta 2007, 1770, 601-608.

4. Cho, Y.; Frazier, A.B.; Chen, Z.G.; Han, A. Whole-cell impedance analysis for highly and poorly metastatic cancer cells. J. Microelectromech. Syst. 2009, 18, 808-817.

5. Zhao, Y.; Chen, D.; Luo, Y.; Li, H.; Deng, B.; Huang, S.-B.; Chiu, T.-K.; Wu, M.-H.; Long, R.; Hu, H.; et al. A microfluidic system for cell type classification based on cellular size-independent electrical properties. Lab Chip 2013, 13, 2272-2277.

6. Byun, S.; Son, S.; Amodei, D.; Cermak, N.; Shaw, J.; Kang, J.H.; Hecht, V.C.; Winslow, M.M.; Jacks, T.; Mallick, P.; et al. Characterizing deformability and surface friction of cancer cells. Proc. Natl. Acad. Sci. USA 2013, 110, 7580-7585.

7. Zhao, Y.; Zhao, X.T.; Chen, D.Y.; Luo, Y.N.; Jiang, M.; Wei, C.; Long, R.; Yue, W.T.; Wang, J.B.; Chen, J. Tumor cell characterization and classification based on cellular specific membrane capacitance and cytoplasm conductivity. Biosens. Bioelectron. 2014, 57, 245-253.

8. Radke, S.M.; Alocilja, E.C. A high density microelectrode array biosensor for detection of E. coli O157:H7. Biosens. Bioelectron. 2005, 20, 1662-1667.

9. Bow, H.; Pivkin, I. V; Diez-Silva, M.; Goldfless, S.J.; Dao, M.; Niles, J.C.; Suresh, S.; Han, J. A microfabricated deformability-based flow cytometer with application to malaria. Lab Chip 2011, 11, 1065-1073.

10. Du, E.; Ha, S.; Diez-Silva, M.; Dao, M.; Suresh, S.; Chandrakasan, A.P. Electric impedance microflow cytometry for characterization of cell disease states. Lab Chip 2013, 13, 3903-3909.

11. Asphahani, F.; Zhang, M. Cellular impedance biosensors for drug screening and toxin detection. Analyst 2007, 132, 835-841.

12. Gabriel, C.; Gabriel, S.; Corthout, E. The dielectric properties of biological tissues: I. Lit. Surv. 1996, 41, 2231-2249.

13. Stoneman, M.R.; Kosempa, M.; Gregory, W.D.; Gregory, C.W.; Marx, J.J.; Mikkelson, W.; Tjoe, J.; Raicu, V. Correction of electrode polarization contributions to the dielectric properties of normal and cancerous breast tissues at audio/radiofrequencies. Phys. Med. Biol. 2007, 52, 6589-6604.

14. Yager, P.; Edwards, T.; Fu, E.; Helton, K.; Nelson, K.; Tam, M.R.; Weigl, B.H. Microfluidic diagnostic technologies for global public health. Nature 2006, 442, 412-418.

15. Whitesides, G.M. The origins and the future of microfluidics. Nature 2006, 442, 368-373.

16. Brehm-Stecher, B.F.; Johnson, E.A. Single-cell microbiology: tools, technologies, and applications. Microbiol. Mol. Biol. Rev. 2004, 68, 538-559.

17. Marcus, J.S.; Anderson, W.F.; Quake, S.R. Microfluidic single-cell mRNA isolation and analysis. Anal. Chem. 2006, 78, 3084-3089.

18. Han, A.; Yang, L.; Frazier, A.B. Quantification of the heterogeneity in breast cancer cell lines using whole-cell impedance spectroscopy. Clin. Cancer Res. 2007, 13, 139-143.

19. Kunzelmann, K. Ion Channels and Cancer. J. Membr. Biol. 2005, 205, 159-173. 
20. Huang, S.-B.; Zhao, Y.; Chen, D.; Lee, H.-C.; Luo, Y.; Chiu, T.-K.; Wang, J.; Chen, J.; Wu, M.-H. A clogging-free microfluidic platform with an incorporated pneumatically driven membrane-based active valve enabling specific membrane capacitance and cytoplasm conductivity characterization of single cells. Sens. Actuators B Chem. 2014, 190, 928-936.

21. Jang, L.-S.; Wang, M.-H. Microfluidic device for cell capture and impedance measurement. Biomed. Microdevices 2007, 9, 737-743.

22. Abdolahad, M.; Sanaee, Z.; Janmaleki, M.; Mohajerzadeh, S.; Abdollahi, M.; Mehran, M. Vertically aligned multiwall-carbon nanotubes to preferentially entrap highly metastatic cancerous cells. Carbon N. Y. 2012, 50, 2010-2017.

23. Katsumoto, Y.; Tatsumi, K.; Doi, T.; Nakabe, K. Electrical classification of single red blood cell deformability in high-shear microchannel flows. Int. J. Heat Fluid Flow 2010, 31, 985-995.

24. Dondorp, A.M.; Kager, P.A.; Vreeken, J.; White, N.J. Abnormal blood flow and red blood cell deformability in severe malaria. Parasitol. Today 2000, 16, 228-232.

25. Zhao, Y.; Inayat, S.; Dikin, D.A.; Singer, J.H.; Ruoff, R.S.; Troy, J.B. Patch clamp technique: review of the current state of the art and potential contributions from nanoengineering. Proc. Inst. Mech. Eng. Part N: J. Nanoeng. Nanosyst. 2008, 222, 1-11.

26. Sakmann, B.; Neher, E. Patch clamp techniques for studying ionic channels in excitable membranes. Annu. Rev. Physiol. 1984, 46, 455-472.

27. Neher, E.; Sakmann, B. The patch clamp technique. Sci. Am. 1992, 266, 44-51.

28. Hamill, O.P.; Marty, A.; Neher, E.; Sakmann, B.; Sigworth, F.J. Improved Patch-clamp techniques for high-resolution current recording from cells and cell-free membrane patches. Pflugers Arch. Eur. J. Physiol. 1981, 391, 85-100.

29. Jain, G.; Muley, N.; Soni, P.; Singh, J.N.; Sharma, S.S. Patch clamp technique: conventional to automated. Curr. Res. Inf. Pharm. Sci. 2009, 10, 9-15.

30. DeFelice, L.J. Electrical Properties of Cells: Patch Clamp for Biologists; Springer Science \& Business Media: New York, NY, USA, 1997.

31. Marty, A.; Neher, E. Tight-seal whole-cell recording. In Single-Channel Recording; Sakmann, B., Neher, E., Eds.; Springer US: Boston, MA, USA, 2009; pp. 31-52.

32. Ogden, D.; Stanfield, P. Patch Clamp techniques for single channel and whole-cell recording. In Microelectrode Techniques; The Company of Biologists Ltd.: Cambridge, UK, 1994; pp. 53-78.

33. Karmazínová, M.; Lacinová, L. Measurement of cellular excitability by whole cell patch clamp technique. Physiol. Res. 2010, 59, S1-S7.

34. Zhang, J.; Davidson, R.M.; Wei, M.D.; Loew, L.M. Membrane electric properties by combined patch clamp and fluorescence ratio imaging in single neurons. Biophys. J. 1998, 74, 48-53.

35. Willumsen, N.J.; Bech, M.; Olesen, S.-P.; Jensen, B.S.; Korsgaard, M.P.G.; Christophersen, P. High throughput electrophysiology: New perspectives for ion channel drug discovery. Recept. Channels 2003, 9, 3-12.

36. Bébarová, M. Advances in patch clamp technique: towards higher quality and quantity. Gen. Physiol. Biophys. 2012, 31, 131-140. 
37. Ahmad, M.R.; Nakajima, M. Single cells electrical characterizations using nanoprobe via ESEM-nanomanipulator system. IEEE Conf. Nanotechnol. 2009, 8, 589-592.

38. Hafiz, A.; Sulaiman, M.; Ahmad, M.R. Rigid and conductive dual nanoprobe for single cell analysis. J. Teknol. 2014, 8, 107-113.

39. Esteban-Ferrer, D.; Edwards, M.A; Fumagalli, L.; Juárez, A.; Gomila, G. Electric polarization properties of single bacteria measured with electrostatic force microscopy. ACS Nano 2014, 8 , 9843-9849.

40. Sulaiman, A.H.M.; Ahmad, M.R. Integrated dual nanoprobe-microfluidic system for single cell penetration. In Proceedings of the 2013 IEEE International Conference on Control System, Computing and Engineering (ICCSCE), Mindeb, 29 November-1 December 2013; pp. 568-572.

41. Unger, M.A.; Chou, H.P.; Thorsen, T.; Scherer, A.; Quake, S.R. Monolithic microfabricated valves and pumps by multilayer soft lithography. Science 2000, 288, 113-116.

42. Hansen, C.; Quake, S.R. Microfluidics in structural biology: Smaller, faster...better. Curr. Opin. Struct. Biol. 2003, 13, 538-544.

43. Reyes, D.R.; Iossifidis, D.; Auroux, P.-A.; Manz, A. Micro total analysis systems. 1. introduction, theory, and technology. Anal. Chem. 2002, 74, 2623-2636.

44. Javanmard, M.; Davis, R.W. A microfluidic platform for electrical detection of DNA hybridization. Sens. Actuators B Chem. 2011, 154, 22-27.

45. Yang, L.; Li, Y.; Griffis, C.L.; Johnson, M.G. Interdigitated microelectrode (IME) impedance sensor for the detection of viable salmonella typhimurium. Biosens. Bioelectron. 2004, 19, 1139-1147.

46. Valencia, P.M.; Farokhzad, O.C.; Karnik, R.; Langer, R. Microfluidic technologies for accelerating the clinical translation of nanoparticles. Nat. Nanotechnol. 2012, 7, 623-629.

47. Tian Wei-Cheng, E.F. Microfluidics for Biological Applications; Springer Science + Business Media, LLC: New York, NY, USA, 2008.

48. Yang, J.; Huang, Y.; Wang, X.; Wang, X.B.; Becker, F.F.; Gascoyne, P.R. Dielectric properties of human leukocyte subpopulations determined by electrorotation as a cell separation criterion. Biophys. J. 1999, 76, 3307-3314.

49. Cen, E.G.; Dalton, C.; Li, Y.; Adamia, S.; Pilarski, L.M.; Kaler, K.V.I.S. A Combined dielectrophoresis, traveling wave dielectrophoresis and electrorotation microchip for the manipulation and characterization of human malignant Cells. J. Microbiol. Methods 2004, 58, 387-401.

50. Arnold, W.M.; Zimmermann, U. Rotating-field-induced rotation and measurement of the membrane capacitance of single mesophyll cells of avena sativa sites. Z. Naturforsch $\mathbf{1 9 8 2}$, $37 c, 908-915$.

51. Dalton, C.; Goater, A.D.; Burt, J. P.H.; Smith, H.V. Analysis of parasites by electrorotation. J. Appl. Microbiol. 2004, 96, 24-32.

52. Huang, J.P.; Yu, K.W. First-principles approach to electrorotation assay. J. Phys. Condens. Matter 2002, 14, 1213-1221. 
53. Huang, J.; Yu, K.; Gu, G. Electrorotation of a pair of spherical particles. Phys. Rev. E 2002, 65, doi:org/10.1103/PhysRevE.65.021401.

54. Jones, T.B. Basic theory of dielectrophoresis and electrorotation. IEEE Eng. Med. Biol. Mag. 2003, 22, 33-42.

55. Morgan, H.; Green, N.G. AC Electrokinetics: Colloids and Nanoparticles; Microtechnologies and Microsystems Series; Research Studies Press: Hertfordshire, UK, 2003.

56. Han, S.-I.; Joo, Y.-D.; Han, K.-H. An electrorotation technique for measuring the dielectric properties of cells with simultaneous use of negative quadrupolar dielectrophoresis and electrorotation. Analyst 2013, 138, 1529-1537.

57. Zheng, Y.; Nguyen, J.; Wei, Y.; Sun, Y. Recent advances in microfluidic techniques for single-cell biophysical characterization. Lab Chip 2013, 13, 2464-2483.

58. Zhou, X.F.; Markx, G.H.; Pethig, R. Effect of biocide concentration on electrorotation spectra of yeast cells. Biochim. Biophys. Acta 1996, 1281, 60-64.

59. Hölzel, R. Non-invasive determination of bacterial single cell properties by electrorotation. Biochim. Biophys. Acta 1999, 1450, 53-60.

60. Dalton, C.; Goater, A.D.; Drysdale, J.; Pethig, R. Parasite viability by electrorotation. Colloids Surfaces A Physicochem. Eng. Asp. 2001, 195, 263-268.

61. Goater, A.D.; Burt, J.P. H.; Pethig, R. A combined travelling wave dielectrophoresis and electrorotation device: Applied to the concentration and viability determination of cryptosporidium. J. Phys. D Appl. Phys. 1997, 30, L65-L69.

62. De Gasperis, G.; Wang, X.; Yang, J.; Becker, F.F.; Gascoyne, P.R.C. Automated electrorotation: dielectric characterization of living cells by real-time motion estimation. Meas. Sci. Technol. 1998, 9, 518-529.

63. Cristofanilli, M.; de Gasperis, G.; Zhang, L.; Hung, M.C. Automated electrorotation to reveal dielectric variations related to HER-2/neu overexpression in MCF-7 sublines. Clin. Cancer Res. 2002, 8, 615-619.

64. Hughes, M.P. Computer-aided analysis of conditions for optimizing practical electrorotation. Phys. Med. Biol. 1998, 43, 3639-3648.

65. Wang, X.B.; Huang, Y.; Gascoyne, P.R.; Becker, F.F.; Hölzel, R.; Pethig, R. Changes in friend murine erythroleukaemia cell membranes during induced differentiation determined by electrorotation. Biochim. Biophys. Acta 1994, 1193, 330-344.

66. Becker, F.F.; Wang, X.B.; Huang, Y.; Pethig, R.; Vykoukal, J.; Gascoyne, P.R. Separation of human breast cancer cells from blood by differential dielectric affinity. Proc. Natl. Acad. Sci. USA 1995, 92, 860-864.

67. Hölzel, R. Electrorotation of single yeast cells at frequencies between $100 \mathrm{~Hz}$ and $1.6 \mathrm{GHz}$. Biophys. J. 1997, 73, 1103-1109.

68. Davey, H.M.; Kell, D.B. Flow cytometry and cell sorting of heterogeneous microbial populations: The Importance of single-cell analyses. Microbiol. Rev. 1996, 60, 641-696.

69. Shapiro, H.M. The evolution of cytometers. Cytom. Part A 2004, 58, 13-20.

70. Givan, A.L. Flow Cytometry: First Principles; John Wiley \& Sons, Inc.: New York, NY, USA, 2001. 
71. WH, C. High speed automatic blood cell counter and cell analyzer. Proc. Natl. Electron. Conf. 1956, 12, 1034-1040.

72. Sun, T.; Morgan, H. Single-cell microfluidic impedance cytometry: A review. Microfluid. Nanofluid. 2010, 8, 423-443.

73. Saleh, O.A.; Sohn, L.L. Quantitative sensing of nanoscale colloids using a microchip coulter counter. Rev. Sci. Instrum. 2001, 72, 4449.

74. Sohn, L.L.; Saleh, O.A.; Facer, G.R.; Beavis, A.J.; Allan, R.S.; Notterman, D.A. Capacitance cytometry: Measuring biological cells one by one. Proc. Natl. Acad. Sci. USA 2000, 97, 10687-10690.

75. Gawad, S.; Schild, L.; Renaud, P.H. Micromachined impedance spectroscopy flow cytometer for cell analysis and particle sizing. Lab Chip 2001, 1, 76-82.

76. Cheung, K.; Gawad, S.; Renaud, P. Impedance spectroscopy flow cytometry: On-chip label-free cell differentiation. Cytom. Part A 2005, 65, 124-132.

77. Sun, T.; Green, N.G.; Gawad, S.; Morgan, H. Analytical electric field and sensitivity analysis for two microfluidic impedance cytometer designs. IET Nanobiotechnol. 2007, 1, 69-79.

78. Schade-Kampmann, G.; Huwiler, A.; Hebeisen, M.; Hessler, T.; di Berardino, M. On-chip non-invasive and label-free cell discrimination by impedance spectroscopy. Cell Prolif. 2008, 41, 830-840.

79. Küttel, C.; Nascimento, E.; Demierre, N.; Silva, T.; Braschler, T.; Renaud, P.; Oliva, A.G. Label-free detection of babesia bovis infected red blood cells using impedance spectroscopy on a microfabricated flow cytometer. Acta Trop. 2007, 102, 63-68.

80. Haandbæk, N.; Bürgel, S.C.; Heer, F.; Hierlemann, A. Characterization of subcellular morphology of single yeast cells using high frequency microfluidic impedance cytometer. Lab Chip 2014, 14, 369-377.

81. Holmes, D.; Pettigrew, D.; Reccius, C.H.; Gwyer, J.D.; van Berkel, C.; Holloway, J.; Davies, D.E.; Morgan, H. Leukocyte analysis and differentiation using high speed microfluidic single cell impedance cytometry. Lab Chip 2009, 9, 2881-2889.

82. Haandbæk, N.; With, O.; Bürgel, S.C.; Heer, F.; Hierlemann, A. Resonance-enhanced microfluidic impedance cytometer for detection of single bacteria. Lab Chip 2014, 14, 3313-3324.

83. Van Berkel, C.; Gwyer, J.D.; Deane, S.; Green, N.G.; Green, N.; Holloway, J.; Hollis, V.; Morgan, H. Integrated systems for rapid point of care (PoC) blood cell analysis. Lab Chip 2011, 11, 1249-1255.

84. Han, X.; van Berkel, C.; Gwyer, J.; Capretto, L.; Morgan, H. Microfluidic lysis of human blood for leukocyte analysis using single cell impedance cytometry. Anal. Chem. 2012, 84, 1070-1075.

85. Evander, M.; Ricco, A.J.; Morser, J.; Kovacs, G.T.A; Leung, L.L.K.; Giovangrandi, L. Microfluidic impedance cytometer for platelet analysis. Lab Chip 2013, 13, 722-729.

86. Myers, F.B.; Zarins, C.K.; Abilez, O.J.; Lee, L.P. Label-free electrophysiological cytometry for stem cell-derived cardiomyocyte clusters. Lab Chip 2013, 13, 220-228. 
87. Emaminejad, S.; Javanmard, M.; Dutton, R.W.; Davis, R.W. Microfluidic diagnostic tool for the developing world: Contactless impedance flow cytometry. Lab Chip 2012, 12, 4499-4507.

88. Sun, T.S.T.; Tsuda, S.; Zauner, K.-P.; Morgan, H. Single cell imaging using electrical impedance tomography. In Proceedings of the 4th IEEE International Conference on Nano/Micro Engineered and Molecular Systems, Shenzhen, China, 5-8 January 2009; pp. 858-863.

89. Chai, K.T.C.; Davies, J.H.; Cumming, D.R.S. Electrical impedance tomography for sensing with integrated microelectrodes on a CMOS microchip. Sens. Actuators B Chem. 2007, 127, 97-101.

90. Benazzi, G.; Holmes, D.; Sun, T.; Mowlem, M.C.; Morgan, H. Discrimination and analysis of phytoplankton using a microfluidic cytometer. IET Nanobiotechnol. 2007, 1, 94-101.

91. Katsumoto, Y.; Hayashi, Y.; Oshige, I.; Omori, S.; Kishii, N.; Yasuda, A.; Asami, K. Dielectric cytometry with three-dimensional cellular modeling. Biophys. J. 2008, 95, 3043-3047.

92. Cheung, K.C.; di Berardino, M.; Schade-Kampmann, G.; Hebeisen, M.; Pierzchalski, A.; Bocsi, J.; Mittag, A.; Tárnok, A. Microfluidic impedance-based flow cytometry. Cytometry. A 2010, 77, 648-666.

93. Bernabini, C.; Holmes, D.; Morgan, H. Micro-impedance cytometry for detection and analysis of micron-sized particles and bacteria. Lab Chip 2011, 11, 407-412.

94. Chen, J.; Zheng, Y.; Tan, Q.; Zhang, Y.L.; Li, J.; Geddie, W.R.; Jewett, M.A.S.; Sun, Y. A microfluidic device for simultaneous electrical and mechanical measurements on single cells. Biomicrofluidics 2011, 5, 14113.

95. Mernier, G.; Duqi, E.; Renaud, P. Characterization of a novel impedance cytometer design and its integration with lateral focusing by dielectrophoresis. Lab Chip 2012, 12, 4344-4349.

96. Zheng, Y.; Shojaei-Baghini, E.; Wang, C.; Sun, Y. Microfluidic characterization of specific membrane capacitance and cytoplasm conductivity of single cells. Biosens. Bioelectron. 2013, 42, 496-502.

97. Han, A.; Frazier, A.B. Ion channel characterization using single cell impedance spectroscopy. Lab Chip. 2006, 6, 1412-1414.

98. Cho, S.; Thielecke, H. Micro Hole-based cell chip with impedance spectroscopy. Biosens. Bioelectron. 2007, 22, 1764-1768.

99. Kurz, C.M.; Büth, H.; Sossalla, A.; Vermeersch, V.; Toncheva, V.; Dubruel, P.; Schacht, E.; Thielecke, H. Chip-based impedance measurement on single cells for monitoring sub-toxic effects on cell membranes. Biosens. Bioelectron. 2011, 26, 3405-3412.

100. Mondal, D.; Roychaudhuri, C.; Das, L.; Chatterjee, J. Microtrap electrode devices for single cell trapping and impedance measurement. Biomed. Microdevices 2012, 14, 955-964.

101. Malleo, D.; Nevill, J.T.; Lee, L.P.; Morgan, H. Continuous differential impedance spectroscopy of single cells. Microfluid. Nanofluid. 2010, 9, 191-198.

102. Ameri, S.K.; Singh, P.K.; Dokmeci, M.R.; Khademhosseini, A.; Xu, Q.; Sonkusale, S.R. All electronic approach for high-throughput cell trapping and lysis with electrical impedance monitoring. Biosens. Bioelectron. 2014, 54, 462-467. 
103. Sano, M.B.; Caldwell, J.L.; Davalos, R.V. Modeling and development of a low frequency contactless dielectrophoresis (cDEP) platform to sort cancer cells from dilute whole blood samples. Biosens. Bioelectron. 2011, 30, 13-20.

104. Tsai, S.-L.; Chiang, Y.; Wang, M.-H.; Chen, M.-K.; Jang, L.-S. Battery-powered portable instrument system for single-cell trapping, impedance measurements, and modeling analyses. Electrophoresis 2014, 16, 2392-2400.

105. Sin, M.L.Y.; Mach, K.E.; Wong, P.K.; Liao, J.C. Advances and challenges in biosensor-based diagnosis of infectious diseases. Expert Rev. Mol. Diagn. 2014, 14, 225-244.

106. Han, K.-H.; Han, A.; Frazier, A.B. Microsystems for isolation and electrophysiological analysis of breast cancer cells from blood. Biosens. Bioelectron. 2006, 21, 1907-1914.

107. Lan, K.C.; Jang, L.S. Integration of single-cell trapping and impedance measurement utilizing microwell electrodes. Biosens. Bioelectron. 2011, 26, 2025-2031.

108. Shah, P.; Zhu, X.; Chen, C.; Hu, Y.; Li, C.-Z. Lab-on-chip device for single cell trapping and analysis. Biomed. Microdevices 2014, 16, 35-41.

109. Klepárník, K.; Foret, F. Recent advances in the development of single cell analysis-A Review. Anal. Chim. Acta 2013, 800, 12-21.

110. Ahmad, I.; Ahmad, M. Trends in characterizing single cell's stiffness properties. Micro Nano Syst. Lett. 2014, 2, doi:10.1186/s40486-014-0008-5.

111. Lewpiriyawong, N.; Kandaswamy, K.; Yang, C.; Ivanov, V.; Stocker, R. Microfluidic characterization and continuous separation of cells and particles using conducting poly(dimethyl siloxane) electrode induced alternating current-dielectrophoresis. Anal. Chem. 2011, 83, 9579-9585.

112. Goater, A.D.; Pethig, R. Electrorotation and dielectrophoresis. Parasitology 1998, 117, S177-S189.

113. Čemažar, J.; Damijan Miklavčič, T.K. Microfluidic devices for manipulation, modification and characterization of biological cells in electric Fields-A Review. J. Microelectron. Electron. Compon. Mater. 2013, 43, 143-161. 


\title{
Microfluidic Impedance Flow Cytometry Enabling High-Throughput Single-Cell Electrical Property Characterization
}

\author{
Jian Chen, Chengcheng Xue, Yang Zhao, Deyong Chen, Min-Hsien Wu and Junbo Wang
}

Abstract: This article reviews recent developments in microfluidic impedance flow cytometry for high-throughput electrical property characterization of single cells. Four major perspectives of microfluidic impedance flow cytometry for single-cell characterization are included in this review: (1) early developments of microfluidic impedance flow cytometry for single-cell electrical property characterization; (2) microfluidic impedance flow cytometry with enhanced sensitivity; (3) microfluidic impedance and optical flow cytometry for single-cell analysis and (4) integrated point of care system based on microfluidic impedance flow cytometry. We examine the advantages and limitations of each technique and discuss future research opportunities from the perspectives of both technical innovation and clinical applications.

Reprinted from Int J. Mol. Sci. Cite as: Chen, J.; Xue, C.; Zhao, Y.; Chen, D.; Wu, M.-H.; Wang, J. Microfluidic Impedance Flow Cytometry Enabling High-Throughput Single-Cell Electrical Property Characterization. Int. J. Mol. Sci. 2015, 16, 9804-9830.

\section{Introduction}

Single-cell electrical properties (e.g., membrane capacitance or cytoplasm resistance) can be utilized as cellular biophysical markers to evaluate cellular status in a label-free manner [1,2]. They have been demonstrated to classify various types of tumor cells [3-5], stem cells [6] and blood cells [7-11].

Conventionally there are mainly three techniques capable of characterizing single-cell electrical properties: dielectrophoresis, patch clamping and electrorotation [12]. Dielectrophoresis is demonstrated to quantify cellular electrical properties by curve fitting of the Clausius-Mossotti factor spectra or cell count spectra. However, since the spectra are not from the measurements of the same cells, only average electrical properties of a cell population can be obtained [3,4,12-17]. Patch-clamp devices characterize the activities of cellular ion channels by sucking a portion of cell membrane into a micropipette tip to form a high electrical resistance seal, enabling the quantification of specific membrane capacitance of single cells (an intrinsic size-independent electrical parameter of cells) [18-24]. In electro-rotation, a rotating electric field is exerted to rotate a suspended single cell as a result of Maxwell-Wagner polarization. By measuring the rotating rate as a function of the applied frequency, this method is capable of collecting membrane permittivity and cytoplasm conductivity of single cells [25-32]. However, patch clamping and electrorotation rely on the precise manipulation and positioning of pipettes (patch clamping) or cells (electrorotation) which is time-consuming and labor-intensive [12,33-36]. This could greatly affect the measurement efficiency and therefore hamper the wide application of using these techniques to acquire statistically-meaningful data. 
Microfluidics is the science and technology on the processing and manipulation of small amounts of fluids $\left(10^{-9}\right.$ to $10^{-18}$ liters $)$ in channels with dimensions of tens of micrometers [37-39]. The micrometer dimension well matches with the size of typical biological calls, making microfluidics an ideal platform for cell studies [40-44]. Based on the advantageous features of microfluidic technologies, microfluidics has been used for characterizing the biochemical (e.g., gene and protein) and/or biophysical properties (mechanical and electrical) of cells at the singlecell level [45-51].

Microfluidics-based devices for the characterization of single-cell electrical properties have been proposed, in which two major approaches, the micro electrical impedance spectroscopy ( $\mu$-EIS) and microfluidic impedance flow cytometry $[12,35,36]$, are commonly used. $\mu$-EIS is a non-invasive approach to characterize immobilized single cells between two electrodes relying on hydrodynamic fluid trapping [52-58], vacuum aspiration [59-65], dielectrophoretic forces [66-69] or surface modifications [70-72]. Although this technique can conduct spectroscopy sweeping on the trapped single cells, it normally suffers from limited throughput and thus might not be suitable for collecting data from large amounts of cells [12,33-36].

Meanwhile, microfluidic impedance flow cytometry has also been demonstrated where single cells are pushed to continuously flow through two microelectrodes in which the impedance data of cells at multiple frequencies are measured [35,36]. Compared to the conventional coulter counters which rely on DC or low-frequency signal for cell size characterization [73-76], the multiple-frequency-based impedance data obtained from the microfluidic impedance flow cytometry enable the characterization of cellular sizes, membrane capacitance and cytoplasm resistance in a high-throughput manner [35,36].

In this review, we focus on the recent advances of the four perspectives of microfluidics-based flow cytometers for single-cell electrical property characterization: (1) early developments of microfluidic impedance flow cytometry for single-cell electrical property characterization; (2) microfluidic impedance flow cytometry with enhanced sensitivity; (3) microfluidic impedance and optical flow cytometry for single-cell analysis and (4) integrated point of care system based on microfluidic impedance flow cytometry (see Table 1).

Table 1. Key developments in the field of microfluidic impedance flow cytometry enabling high-throughput cellular electrical property characterization.

\begin{tabular}{|c|c|c|c|}
\hline Techniques & Quantified Parameters & $\begin{array}{c}\text { Classified Objects } \\
\text { and Key Observations }\end{array}$ & References \\
\hline Coplanar microelectrodes & $\begin{array}{l}\text { Two-frequency impedance data } \\
\qquad(1.7 \text { and } 15.0 \mathrm{MHz})\end{array}$ & $\begin{array}{c}\text { Polymer beads of } 5 \text { and } 8 \mu \mathrm{m} \text {, normal } \\
\text { erythrocytes and their ghost } \\
\text { counterparts }\end{array}$ & {$[77]$} \\
\hline Coplanar microelectrodes & $\begin{array}{c}\text { One-frequency impedance } \\
\text { data }(100 \mathrm{kHz})\end{array}$ & $\begin{array}{c}\text { Liver tumor cells at normal, apoptotic } \\
\text { and necrotic status, leukemia cells }\end{array}$ & {$[78]$} \\
\hline Coplanar microelectrodes & $\begin{array}{l}\text { One-frequency impedance } \\
\text { data }(2.0 \mathrm{MHz})\end{array}$ & $\begin{array}{c}\text { Different stages of } P \text {. falciparum } \\
\text { infected red blood cells and uninfected } \\
\text { red blood cells }\end{array}$ & [9] \\
\hline
\end{tabular}


Table 1. Cont.

\begin{tabular}{|c|c|c|c|}
\hline Techniques & Quantified Parameters & $\begin{array}{c}\text { Classified Objects } \\
\text { and Key Observations }\end{array}$ & References \\
\hline Parallel microelectrodes & $\begin{array}{c}\text { Two-frequency impedance } \\
\text { opacity }\left|Z_{\text {high }} /\right| Z_{\text {ref }} \mid\left(f_{\text {ref }}=602\right. \\
\mathrm{kHz}, f_{\text {high }}=350 \mathrm{kHz}-20.0 \\
\mathrm{MHz})\end{array}$ & $\begin{array}{l}\text { Polymer beads of } 5,6 \mu \mathrm{m} \text {, red blood } \\
\text { cells and their fixed counterparts }\end{array}$ & [79] \\
\hline Parallel microelectrodes & $\begin{array}{c}\text { Two-frequency impedance } \\
\text { opacity }\left|\mathrm{Z}_{\text {high }} / / \mathrm{Z}_{\text {ref }}\right|\left(\mathrm{f}_{\text {ref }}=500\right. \\
\left.\mathrm{kHz}, \mathrm{f}_{\text {high }}=0.5-250.0 \mathrm{MHz}\right)\end{array}$ & $\begin{array}{l}\text { Wild-type yeasts and a mutant with } \\
\text { different sizes and distribution of } \\
\text { vacuoles in the intracellular fluid }\end{array}$ & [80] \\
\hline $\begin{array}{l}\text { Parallel microelectrodes }+ \\
\text { insulating fluid focusing }\end{array}$ & $\begin{array}{l}\text { One-frequency impedance } \\
\text { data }(503 \mathrm{kHz})\end{array}$ & Polymer beads of $1,2 \mu \mathrm{m}$, and $E$ coli & [81] \\
\hline $\begin{array}{c}\text { Parallel microelectrodes }+ \\
\text { resonance }\end{array}$ & $\begin{array}{c}\text { Two-frequency impedance data } \\
(87.2 \text { and } 89.2 \mathrm{MHz})\end{array}$ & $\begin{array}{l}\text { E. coli, B. subtilis and } \\
\text { polymer beads of } 2 \mu \mathrm{m}\end{array}$ & [82] \\
\hline Constriction channel & $\begin{array}{c}\text { One-frequency } \\
\text { impedance data }(100 \mathrm{kHz})\end{array}$ & $\begin{array}{l}\text { Size-comparable tumor cells and } \\
\text { their more malignant counterparts }\end{array}$ & [83] \\
\hline Constriction channel & $\begin{array}{c}\text { One-frequency } \\
\text { impedance data }(100 \mathrm{kHz})\end{array}$ & $\begin{array}{l}\text { Adult red blood cells and } \\
\text { neonatal red blood cells }\end{array}$ & [84] \\
\hline Constriction channel & $\begin{array}{c}\text { Four-frequency impedance } \\
\text { data }(50 \mathrm{kHz}, 250 \mathrm{kHz}, \\
500 \mathrm{kHz} \text { and } 1.0 \mathrm{MHz})\end{array}$ & $\begin{array}{c}\text { Polymer beads of } 20 \mu \mathrm{m} \text {, } \\
\text { undifferentiated stem cells and } \\
\text { differentiated stem cells }\end{array}$ & [6] \\
\hline $\begin{array}{l}\text { Constriction channel }+ \\
\text { equivalent circuit model }\end{array}$ & $\begin{array}{l}\text { Specific membrane capacitance } \\
\text { and cytoplasm conductivity }\end{array}$ & $\begin{array}{l}\text { Characterization of size-independent } \\
\text { intrinsic cellular electrical properties } \\
\text { from hundreds of single cells }\end{array}$ & {$[85]$} \\
\hline $\begin{array}{l}\text { Constriction channel + } \\
\text { equivalent circuit model }\end{array}$ & $\begin{array}{l}\text { Specific membrane capacitance } \\
\text { and cytoplasm conductivity }\end{array}$ & $\begin{array}{l}\text { Paired high- and low-metastatic cancer } \\
\text { cells, and tumor cells with single } \\
\text { oncogenes under regulation }\end{array}$ & [5] \\
\hline $\begin{array}{l}\text { Parallel microelectrodes }+ \\
\text { optical lens }\end{array}$ & $\begin{array}{c}\text { Two-frequency impedance data } \\
\text { (503 kHz and } 1.7 \mathrm{MHz} \text { ) and } \\
\text { fluorescent signals }\end{array}$ & $\begin{array}{l}\text { lymphocytes, monocytes } \\
\text { and neutrophils }\end{array}$ & [10] \\
\hline $\begin{array}{l}\text { Parallel microelectrodes }+ \\
\text { optical lens }\end{array}$ & $\begin{array}{c}\text { Two-frequency impedance data } \\
(503 \mathrm{kHz} \text { and } 10.0 \mathrm{MHz}) \text { and } \\
\text { fluorescent signals }\end{array}$ & $\begin{array}{c}\text { Lymphocytes, lymphocytes }+\mathrm{CD} 4 \\
\text { beads, granulocytes, monocytes and } \\
\text { monocytes }+\mathrm{CD} 4\end{array}$ & [11] \\
\hline $\begin{array}{l}\text { Parallel microelectrodes }+ \\
\text { on-chip optical fibers }\end{array}$ & $\begin{array}{c}\text { One-frequency impedance data } \\
(1.0 \mathrm{MHz}) \text {, fluorescent signals, } \\
\text { and side scattered light }\end{array}$ & $\begin{array}{c}\text { Microbeads (10 and } 15 \mu \mathrm{m} \\
\text { diameter fluorescent, } 20 \text { and } 25 \mu \mathrm{m} \\
\text { diameter plain) }\end{array}$ & [86] \\
\hline $\begin{array}{l}\text { Parallel microelectrodes }+ \\
\text { on-chip waveguides }\end{array}$ & $\begin{array}{l}\text { Two-frequency impedance data } \\
\text { (500 kHz and } 2.0 \mathrm{MHz}), \\
\text { fluorescent signals, } \\
\text { and side scattered light }\end{array}$ & $\begin{array}{l}\text { Lymphocytes, granulocytes, } \\
\text { monocytes, neutrophils and CD4 } \\
\text { labelled white blood cells }\end{array}$ & [87] \\
\hline $\begin{array}{l}\text { Parallel microelectrodes }+ \\
\text { sample pretreatment module }\end{array}$ & $\begin{array}{c}\text { Two-frequency impedance data } \\
(500 \mathrm{kHz} \text { and } 1.7 \mathrm{MHz})\end{array}$ & $\begin{array}{l}\text { Lymphocytes, monocytes, neutrophils, } \\
\text { red blood cells and platelets }\end{array}$ & {$[88]$} \\
\hline $\begin{array}{l}\text { Parallel microelectrodes }+ \\
\text { sample pretreatment module }\end{array}$ & $\begin{array}{c}\text { Two-frequency impedance data } \\
(303 \mathrm{kHz} \text { and } 1.7 \mathrm{MHz})\end{array}$ & $\mathrm{CD}^{+}$and $\mathrm{CD} 8^{+}$lymphocytes & [7] \\
\hline
\end{tabular}




\section{Early Development of Microfluidic Flow Cytometry for Single-Cell Electrical Property Characterization}

Renaud et al. are the pioneers in the field of microfluidic impedance flow cytometry [77,79,89-93]. In 2001, Renaud et al. proposed the first microfluidics-based impedance flow cytometry for high-throughput single-cell electrical property characterization [77]. As shown in Figure 1a, a microfluidic chip with channels integrated with a differential pair of coplanar microelectrodes was used to characterize electrical properties of single cells. The cells were flushed through the measurement area in a high-throughput manner with the impedance data measured at two given frequencies. In this study, an equivalent circuit model for microfluidic impedance flow cytometry was developed where $\mathrm{C}_{\mathrm{m}}, \mathrm{R}_{\mathrm{c}}, \mathrm{R}_{\mathrm{sol}}$ and $\mathrm{C}_{\mathrm{dl}}$ represent cell membrane capacitance, cytoplasm resistance, buffer solution resistance and electrical double layer capacitance, respectively (see Figure 1a).

In addition, complex impedance spectrum of a cell as simulated using an equivalent circuit model was shown in Figure 1b. Based on simulation results, the authors suggested that the impedance data for frequencies lower than $100 \mathrm{kHz}$, between $100 \mathrm{kHz}-1 \mathrm{MHz}, 2-5 \mathrm{MHz}$ and $10-100 \mathrm{MHz}$ reflect the electrical double layer, cellular size, membrane capacitance and cytoplasm resistance, respectively. Note that this impedance spectrum has served as the guiding rule of frequency choice in the subsequent development of microfluidic impedance flow cytometry.

To demonstrate its applications, the microfluidic device was used to differentiate latex beads of 5 and $8 \mu \mathrm{m}$ at $1.72 \mathrm{MHz}$. The result confirmed that impedance data at $\sim 1 \mathrm{MHz}$ does reflect particle sizes (see Figure 1c). Furthermore, normal erythrocytes and erythrocyte ghost cells (namely the erythrocytes with cytoplasm replaced with phosphate buffer solution) were characterized and differentiated. The impedance data for these two types of cells were found similar at $1.72 \mathrm{MHz}$ indicating comparable cell sizes whereas, significantly different at $15 \mathrm{MHz}$ suggesting differences in cytoplasm conductivity (see Figure 1d).

In 2005, Renaud et al. proposed the second-generation microfluidic impedance flow cytometry [79] where the parallel overlap microelectrodes were used to replace the previously reported coplanar microelectrode, enabling the production of more homogeneous current density around the single cells under measurement (see Figure 2a). Furthermore, systematic experiments were conducted to classify polystyrene beads ( 5 and $6 \mu \mathrm{m})$, normal red blood cells and fixed red blood cells based on the impedance data at the frequency of $602 \mathrm{kHz}$ and $10 \mathrm{MHz}$ (see Figure $2 \mathrm{~b}$ ). 


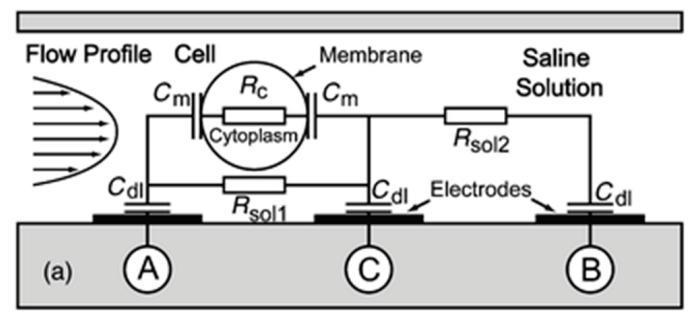

(a)

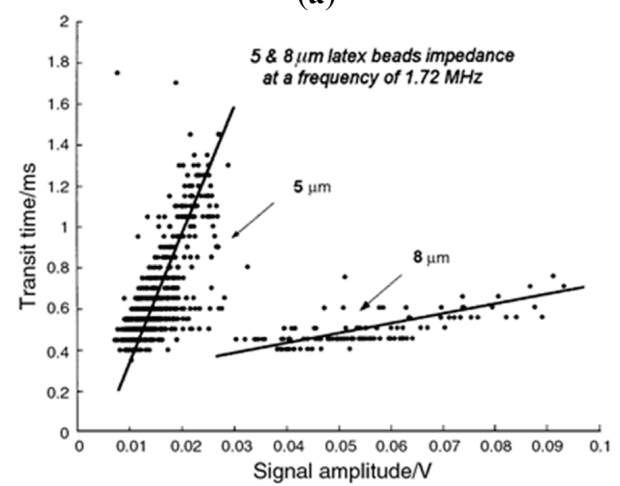

(c)

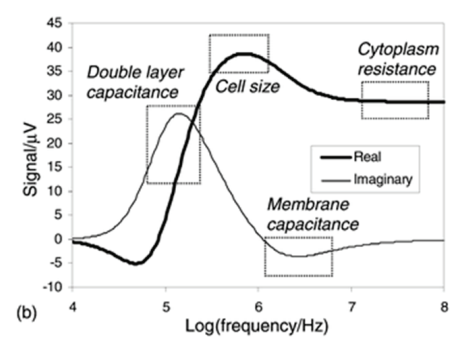

(b)

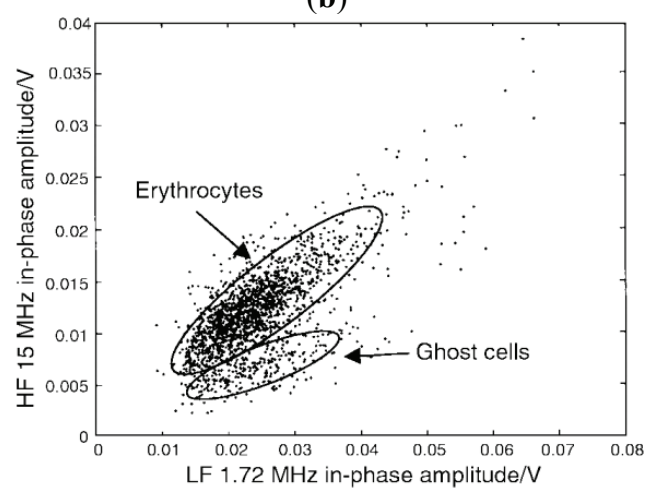

(d)

Figure 1. (a) The first-generation microfluidic impedance flow cytometry where a microfluidic chip with integrated channels and a differential pair of coplanar microelectrodes were proposed to quantify two-frequency impedance data of single cells flushed through the measurement area in a high-throughput manner; (b) The complex impedance spectrum of a cell is simulated using an equivalent circuit model where impedance data at various frequency domains indicate the electrical double layer, cellular size, membrane capacitance and cytoplasm resistance, respectively; (c) Impedance amplitude difference of 5 and $8 \mu \mathrm{m}$ latex beads, confirming that impedance data at $\sim 1 \mathrm{MHz}$ can reflect particle sizes. Note that "transit time" indicates the traveling velocity of latex beads which were also obtained from impedance data; (d) Normal erythrocytes and erythrocyte ghost cells were characterized, with comparable low-frequency impedance data indicating size comparability and significant differences at high-frequency impedance data suggesting cytoplasm conductivity differences [77]. 


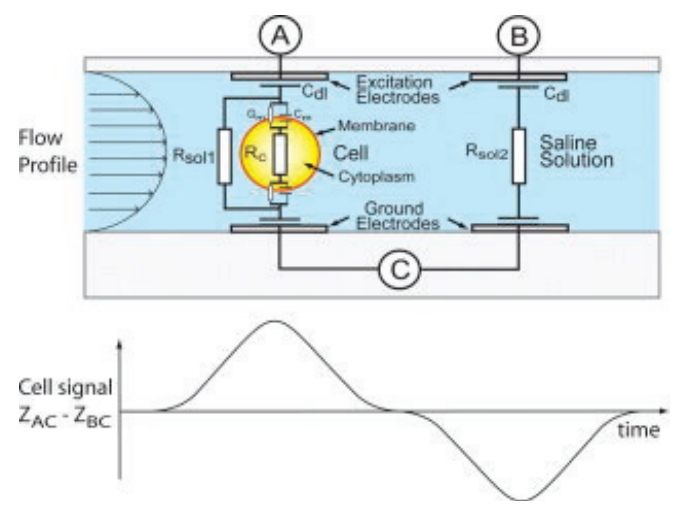

(a)

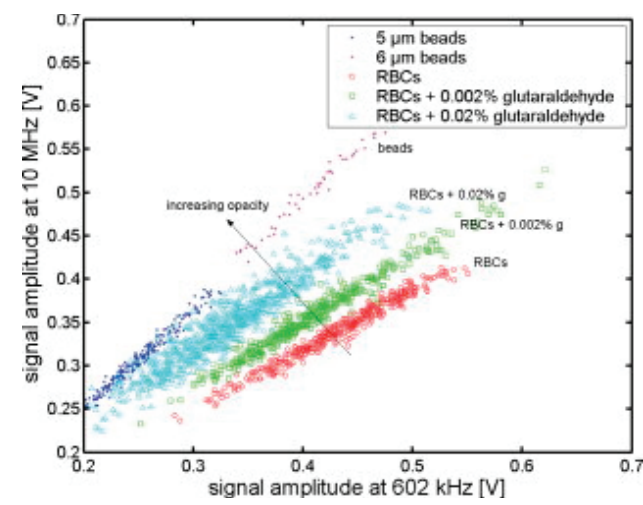

(b)

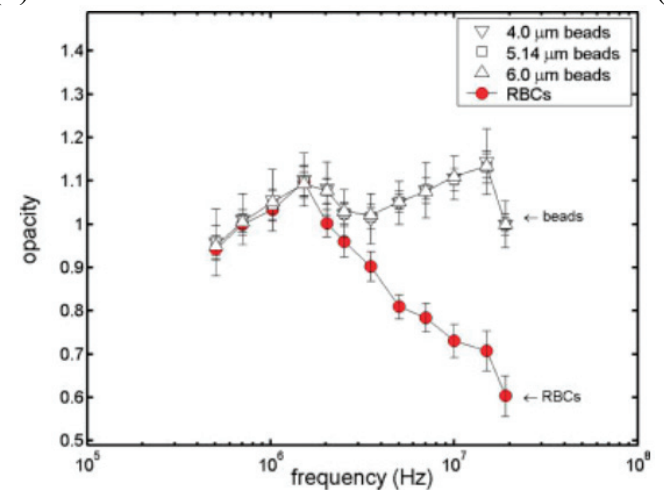

(c)

Figure 2. (a) The second-generation microfluidic impedance flow cytometry where the parallel overlap micro electrodes were used to replace the previously reported coplanar micro electrodes; (b) Two-frequency impedance data of polystyrene beads, normal red blood cells and fixed red blood cells, which can be classified to an extent based on opacity defined as $\left|Z_{\text {high }}\right|\left|Z_{\text {ref }}\right|$; (c) Opacity spectrum of red blood cells and polystyrene beads where no significant difference was noticed among the opacity spectra for polystyrene beads of different diameters, confirming that opacity can be used to normalize the particle size. In addition, a decrease in opacity at the high frequency domain of red blood cells compared to polystyrene beads was observed, confirming that the cytoplasm of red blood cells is more conductive than polystyrene beads [79].

In this study, opacity was defined as $\left|Z_{\text {high }}\right| /\left|Z_{\text {ref }}\right|$ to partially remove the dependence of the impedance data on particle sizes. As shown Figure 2c, no significant difference was noticed among the opacity spectrum $\left(f_{\mathrm{ref}}=602 \mathrm{kHz}\right)$ ) for polystyrene beads of 4.0, 5.1, and $6.0 \mu \mathrm{m}$ diameters, confirming that, to an extent, opacity is insensitive to particle sizes. In addition, a decrease in opacity at the high frequency domain of red blood cells compared to polystyrene beads was observed, confirming that the cytoplasm of red blood cells is more conductive than polystyrene beads. As a valuable impedance parameter, opacity has been commonly used in the subsequent 
development of microfluidic impedance flow cytometry to evaluate electrical properties of single cells.

\section{Microfluidic Impedance Flow Cytometry with Enhanced Sensitivity}

The drawback of the microfluidic impedance flow cytometry reported by Renaud et al. is the lack of close contact between cells and electrodes when the cells were continuously flushed to flow through the detection area between two electrodes. This issue could lead to current leakage where electric signals circumvent the cells under measurement by travelling through solutions surrounding the cells. In addition, the relative positions of travelling single cells between two facing electrodes (i.e., in the middle of two facing electrodes $v$ s. alongside the boundary of one detecting electrode) can also lead to issues of low detection stability and repeatability. In order to address these issues, the detection area of the microfluidic impedance flow cytometry needs to be further reduced. Two approaches have been developed to this end: sandwiching cells between two insulating fluid layers (e.g., insulating fluid flow [81,94]) or confining cells within solid constriction channels (cross sectional area smaller than biological cells) $[63,83,84]$.

As the first demonstration, Morgan et al. developed a microfluidic impedance flow cytometer which utilized an insulating fluid to hydrodynamically focus a sample stream of cells suspended in electrolyte through the sensing area between two microelectrodes [81] (see Figure 3a). The focusing technique enhanced the measurement sensitivity without reducing the dimensions of the microfluidic channels so that channel blockage can be avoided. This microfluidic platform was used to successfully classify polystyrene beads with diameters of 1 and $2 \mu \mathrm{m}$ based on impedance amplitudes at the frequency of $503 \mathrm{kHz}$ (see Figure 3b). As to the classification of $2 \mu \mathrm{m}$ diameter polystyrene beads and $E$. coli $(\sim 2 \mu \mathrm{m}$ in length and $0.5 \mu \mathrm{m}$ in width), a significant overlap in the impedance amplitude histogram was observed, which may result from the comparable sizes between $2 \mu \mathrm{m}$ beads and $E$. coli (see Figure $3 b$ ). In this study, only one frequency at $503 \mathrm{kHz}$ was used, which was previously demonstrated as the frequency enabling particle size quantification [77,79]. More frequencies higher than $503 \mathrm{kHz}$ may be further used to characterize the electrical properties of E. coli.

Although this technique can, to an extent, address the current leakage problem by sandwiching the detection solution between two insulating fluid flows, this type of sandwiching is only one dimensional and produces a vertical conductive sheet of cells (see Figure 3a, top view and side view), which still suffers from the current leakage. In addition, the impedance data of cells can also be affected by the z-direction position of single cells during the measurement [95], which leads to additional concerns on the measurement accuracy in this microfluidic system.

To tackle the technical hurdle, a constriction channel design was put forward to further decrease the current leakage and enable single-cell electrical property characterization $[63,65,83,84]$. The constriction channel design with a cross-section area smaller than that of biological cells was initially used for the characterization of cellular mechanical properties (e.g., red blood cells [84,96-98], white blood cells [99] and tumor cells [83,100-102]), by which single cells were aspirated into the constriction channel with their entry times adopted as a biophysical marker. 

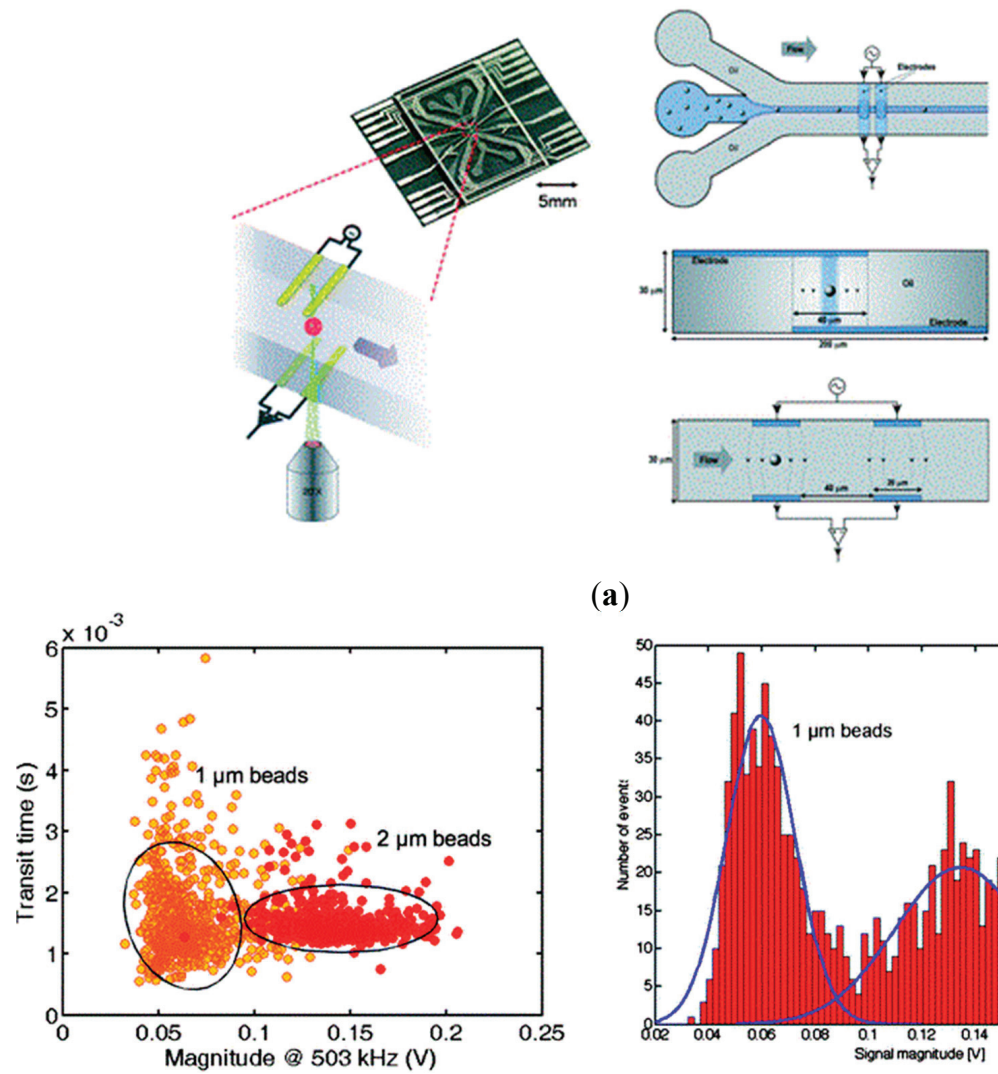

(a)

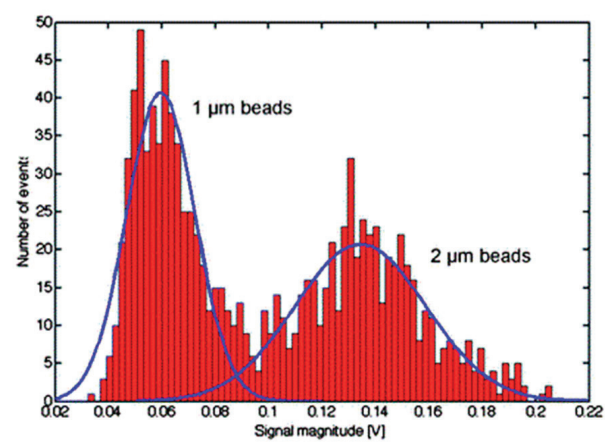

(b)
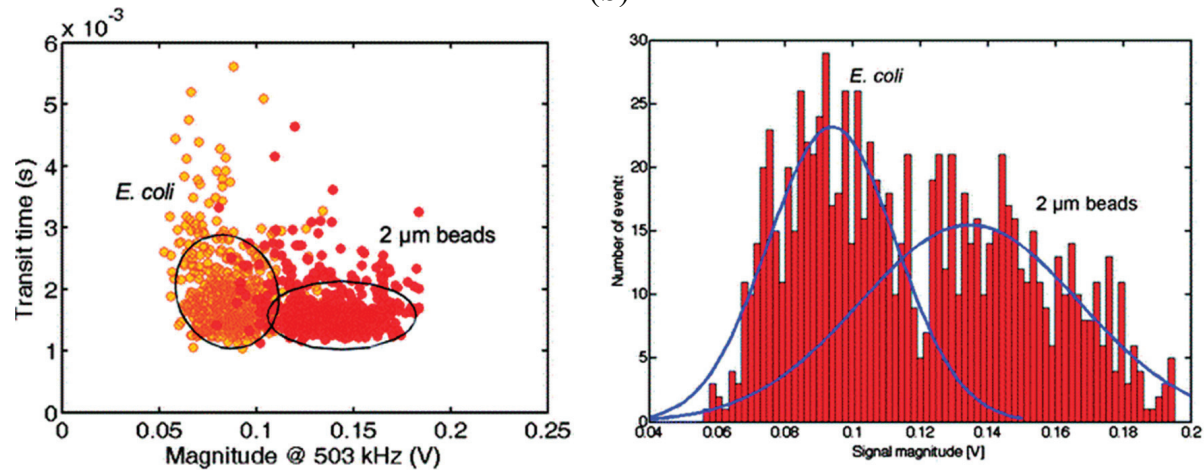

(c)

Figure 3. (a) A microfluidic impedance flow cytometer uses an insulating fluid to hydrodynamically focus a sample stream of cells suspended in electrolyte through the sensing area of two microelectrodes; (b) Successful classification of 1 and $2 \mu$ m diameter polystyrene beads based on impedance amplitudes at $503 \mathrm{kHz}$; (c) As to the classification of $2 \mu \mathrm{m}$ diameter polystyrene beads and $E$ coli ( $\sim 2 \mu \mathrm{m}$ in length and $0.5 \mu \mathrm{m}$ in width), a significant overlap in the impedance amplitude histogram at $503 \mathrm{kHz}$ was observed [81]. 
In 2011, Sun et al. proposed the first microfluidic impedance flow cytometry based on the constriction channel design, where single cells were continuously aspirated through the constriction channel while cell elongations and single-frequency impedance profiles were measured simultaneously (see Figure 4a) [83]. When a cell is aspirated through the constriction channel, it blocks electric fields and leads to a higher impedance amplitude value, which is used as an indicator of cellular electrical properties (see Figure $4 \mathrm{~b}$ ). This technique was used to classify two types of bone cells (osteoblasts $v$ s. osteocytes) using a constriction channel of $6 \mu \mathrm{m} \times 6 \mu \mathrm{m}$ in dimensions (at $100 \mathrm{kHz}$ ). To quantify the overall impedance of the cell, an impedance amplitude ratio was adopted which is defined as the ratio between the highest impedance amplitude value captured while cells are squeezed through the constriction channel and the background impedance amplitude value without cells. Compared with osteocytes, osteoblasts were found to have a larger cell elongation length and a higher impedance amplitude ratio (see Figure 4c).

The constriction channel design $(8 \mu \mathrm{m} \times 8 \mu \mathrm{m}$ at $100 \mathrm{kHz})$ was then used to characterize tumor cells EMT6 and their more malignant counterparts EMT6/AR 1.0, revealing a linear trend between the cell elongation length and the impedance amplitude ratio with different slopes and different $y$-axis intersections (see Figure 4d).

Furthermore, based on equivalent circuit models and two-frequency measurements, these raw impedance data were translated to intrinsic cellular electrical parameters including specific membrane capacitance $\left(\mathrm{C}_{\text {specific membrane }}\right)$ and cytoplasm conductivity $\left(\sigma_{\text {cytoplasm}}\right)$ [5,85,103,104]. As shown in Figure 5a, when a cell is squeezed into the constriction channel, there is an increase in amplitude and a decrease in phase for the impedance data at the frequency of 1 and $100 \mathrm{kHz}$. The 1 $\mathrm{kHz}$ impedance data was used to evaluate cellular sealing properties with constriction channel walls to obtain Rleak while $100 \mathrm{kHz}$ impedance data was used to quantify $\mathrm{C}_{\text {membrane }}$ and $\mathrm{R}_{\text {cytoplasm, }}$, which were then translated to $C_{\text {specific membrane }}$ and $\sigma_{\text {cytoplasm }}$ (see Figure 5a) [85]. Based on the above translations, the $\mathrm{C}_{\text {specific membrane }}$ and $\sigma_{\text {cytoplasm }}$ of tumor cells with different types were quantified [5].

For paired high- and low-metastatic carcinoma strains 95D and 95C cells, significant differences in both $\mathrm{C}_{\text {specific membrane }}$ and $\sigma_{\text {cytoplasm }}$ were observed (see Figure $5 \mathrm{~b}$ ). In addition, a statistically

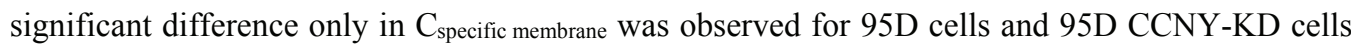
with single oncogene $\mathrm{CCNY}$ down regulation (CCNY is a membrane-associated protein) (see Figure 5c). Furthermore, a statistically significant difference only in $\sigma_{\text {cytoplasm }}$ was observed for A549 cells and A549 CypA-KD cells with single oncogene CypA down regulation (CypA is a cytosolic protein) (see Figure 5d).

Although the combination of impedance flow cytometry with the constriction channel design can adequately tackle the current leakage issue, the use of constriction channel could reduce the detection throughput and may lead to channel blockage. Thus, the detection throughput in the constriction channel-based microfluidic impedance flow cytometry is normally lower than its conventional counterparts $[77,79,81]$. 


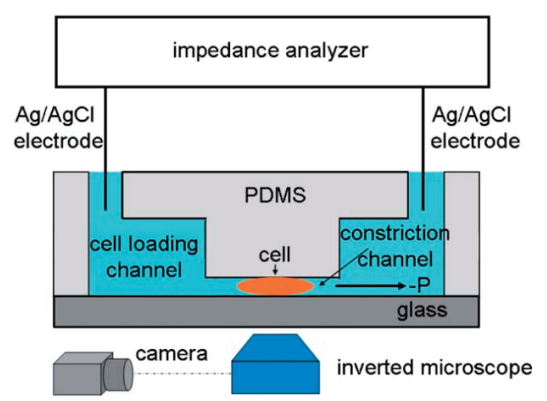

(a)

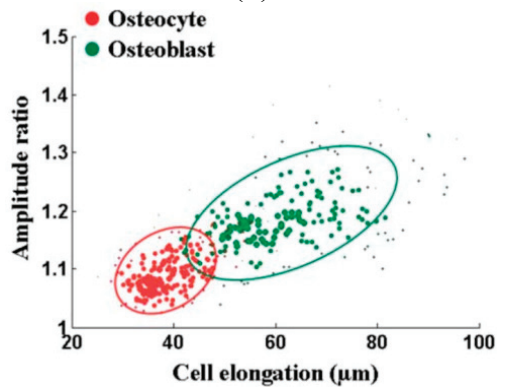

(c)

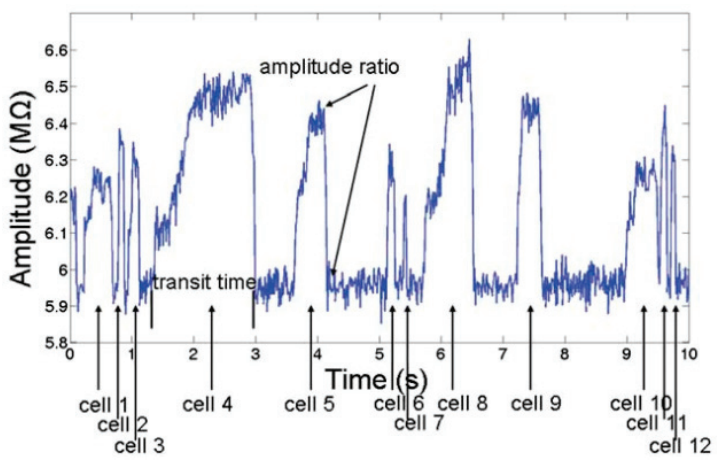

(b)

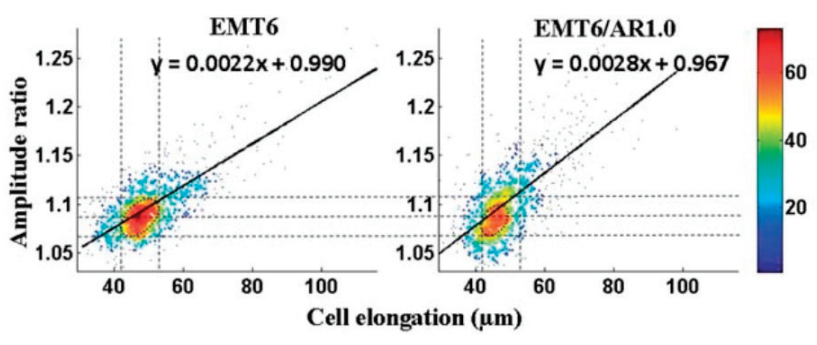

(d)

Figure 4. (a) The constriction channel based microfluidic impedance flow cytometry where single cells were aspirated through a constriction continuously while cell elongations and single frequency impedance profiles are measured simultaneously; (b) Raw impedance data of single cells, recording higher impedance amplitudes during cellular squeezing through the constriction channel; (c) The scatter plot of impedance amplitude ratio vs. cell elongation length for osteocytes and osteoblasts. Compared with osteocytes, osteoblasts have a larger cell elongation length and a higher impedance amplitude ratio; (d) The scatter plot of impedance amplitude ratio $v$ s. cell elongation length for tumor cell EMT6 and their more malignant counterparts EMT6/AR 1.0, revealing a linear trend between cell elongation length and impedance amplitude ratio with different slopes and different $y$-axis intersections Reproduced by permission of the Royal Scoeity of Chemistry [83]. 


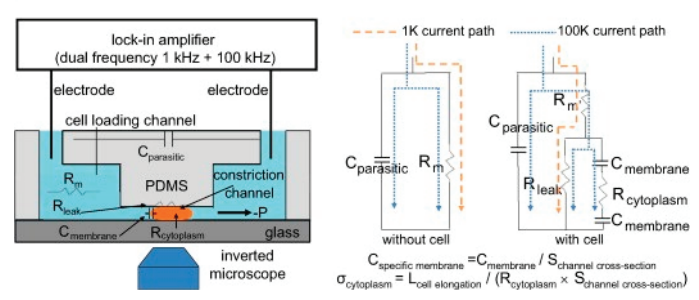

(a)

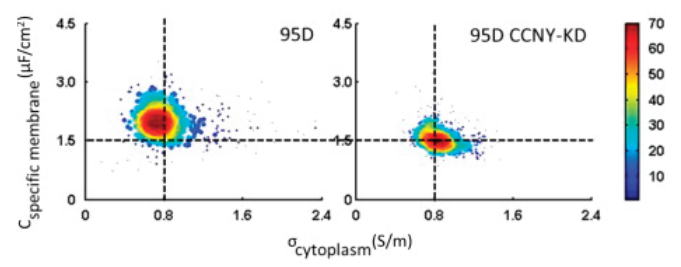

(c)

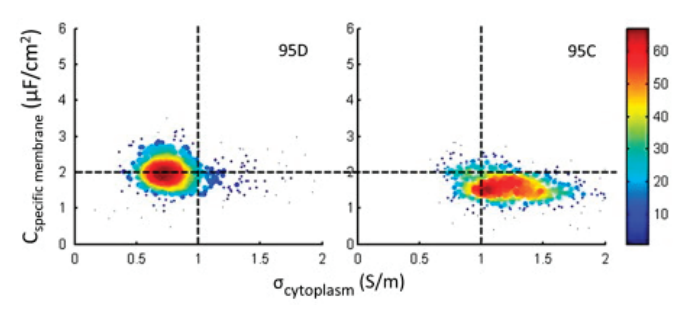

(b)

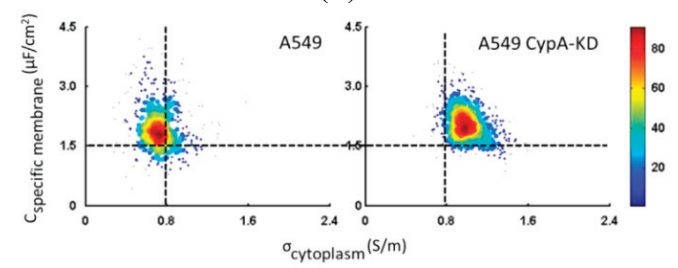

(d)

Figure 5. (a) The microfluidic impedance flow cytometry for continuous characterization of specific membrane capacitance ( $\left.\mathrm{C}_{\text {specific membrane }}\right)$ and cytoplasm conductivity $\left(\sigma_{\text {cytoplasm}}\right)$ of single cells. Cells are aspirated continuously through the constriction channel with impedance data at 1 and $100 \mathrm{kHz}$ measured simultaneously where $1 \mathrm{kHz}$ impedance data were used to evaluate cellular sealing properties with constriction channel walls while $100 \mathrm{kHz}$ impedance data were used to quantify $\mathrm{C}_{\text {specific }}$ membrane and $\sigma_{\text {cytoplasm }}[85]$; (b) For paired high- and low-metastatic carcinoma strains 95D and $95 \mathrm{C}$ cells, significant differences in both $\mathrm{C}_{\text {specific membrane }}$ and $\sigma_{\text {cytoplasm were observed; (c) }}$

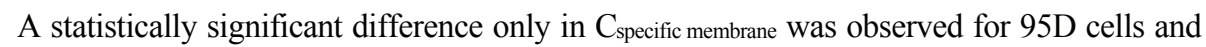
95D CCNY-KD cells with single oncogene CCNY down regulation (CCNY is a membrane-associated protein); (d) A statistically significant difference only in $\sigma_{\text {cytoplasm }}$ was observed for A549 cells and A549 CypA-KD cells with single oncogene CypA down regulatio $\mathrm{n}$ (CypA is a cytosolic protein) [5].

\section{Microfluidic Impedance and Fluorescent Flow Cytometry for Single-Cell Analysis}

In order to enhance the functionality of microfluidic impedance flow cytometry and provide a more comprehensive understanding on cellular biophysical and biochemical properties, Morgan et al. integrated the functions of impedance measurement and fluorescence detection in a microfluidic impedance and fluorescent flow cytometry [8,10,11,35,81,86-88,95,105-117]. Figure 6a shows the first-generation microfluidic platforms capable of characterizing both cellular electrical and optical property consisting of dual laser excitation, three color detection and dual frequency impedance measurement [10]. As the first demonstration, whole blood cells were successfully classified by the microfluidic impedance and fluorescent flow cytometry (see Figure 6b) [10]. In this study, the lymphocytes were differentiated from monocytes and neutrophils due to their significantly smaller cell sizes based on impedance data at $503 \mathrm{kHz}$. In addition, the neutrophils were differentiated from 
monocytes due to their significant differences in membrane capacitance based on impedance data at $1.707 \mathrm{MHz}$.

Furthermore, the whole blood cells mixed with CD4 antibody coated beads were successfully characterized by the microfluidic impedance (frequency: $503 \mathrm{kHz}$ and $10 \mathrm{MHz}$ ) and fluorescent flow cytometry. In this work, the lymphocytes, lymphocytes + CD4 beads, granulocytes \& monocytes and monocytes $+\mathrm{CD} 4$ beads were classified. This method was found useful for CD4 T-lymphocyte counting (see Figure 6c) [11]. Note that these impedance based cell type classification were confirmed by the simultaneous fluorescent measurement.

In the first-generation microfluidics-based impedance and fluorescent flow cytometry, laser excitation and fluorescent collection was implemented by using optical lens and thus only fluorescent signals can be obtained while other optical parameters such as side scattered light cannot be acquired. In order to address the limitation, Morgan et al. proposed the secondgeneration impedance and fluorescent flow cytometry where optical fibers were integrated into the microfluidic platform enabling the simultaneous measurement of impedance signals (at two frequencies) and optical signals (e.g., side scattered light and fluorescence) [86,87]. Figure 7a shows the microfluidic impedance flow cytometry with on-chip optical components. More specifically, a groove in SU-8 material holds a fiber to launch incident light perpendicular to the channel, which is focused into a sheet across the width of the channel using an air compound lens. Fluorescent emission is then collected with the fibers placed in two grooves on the same side as the incident light (at $135^{\circ}$ ). A $7^{\circ}$ fiber is used to measure the optical extinction signal and the light loss due when a particle passes through an incident beam. Furthermore, two more collection fibers placed at $22.5^{\circ}$ and $45^{\circ}$ were designed to measure side scattered light [86]. The reported microfluidic platform was used to classify a mixture of beads (the fluorescent beads with 10 and 15 $\mu \mathrm{m}$ in diameter, and the plain beads with 20 and $25 \mu \mathrm{m}$ in diameter). Figure $7 \mathrm{~b}$ shows that the beads with four different sizes can be distinguished from optical side scattered light, but the impedance signals provide much better discrimination between the populations. The fluorescence signals from the 10 and $15 \mu \mathrm{m}$ beads provide easy discrimination in this platform.

In 2014, Spencer and Morgan proposed a novel microfluidics-based impedance and fluorescent flow cytometry capable of measuring four different parameters, namely fluorescence, large angle side scattered light and dual frequency electrical impedance (electrical volume and opacity) (see Figure 7c) [87]. In this study, on-chip waveguides were used to replace the inserted fibers described in the previous study, which can effectively address the issues of optical fiber misalignments and incident light scattering from multiple interfaces. In addition, a sheath-less particle focusing technique was used and thus hydrodynamic focusing is no longer required. Figure 7d shows a 3-D scatter plot for a CD4 labelled white blood cell sample based on parameters of side scattered light, fluorescence, and two-frequency impedance data. Both side scattered light and low frequency impedance data at $0.5 \mathrm{MHz}$ provide information on cell sizes, which separate smaller lymphocytes from granulocytes. High-frequency impedance data at $2 \mathrm{MHz}$ separate monocytes from neutrophils due to differences in cell membrane capacitance while CD4 labelled white blood cells were distinguished from white blood cells without CD4 labelling based on fluorescent data. 


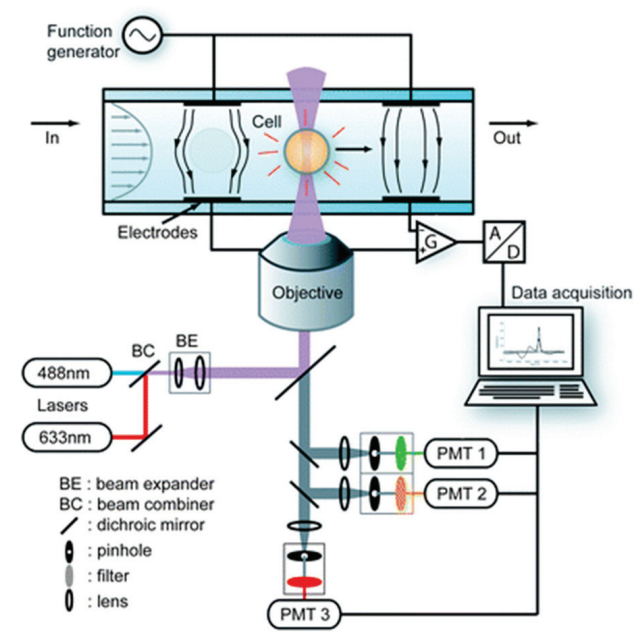

(a)

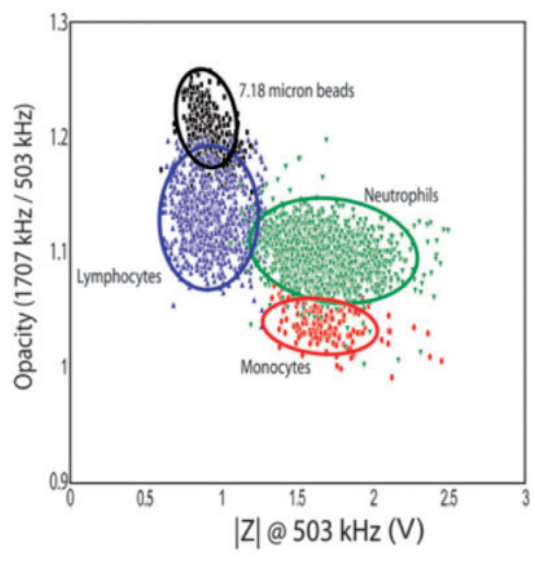

(b)

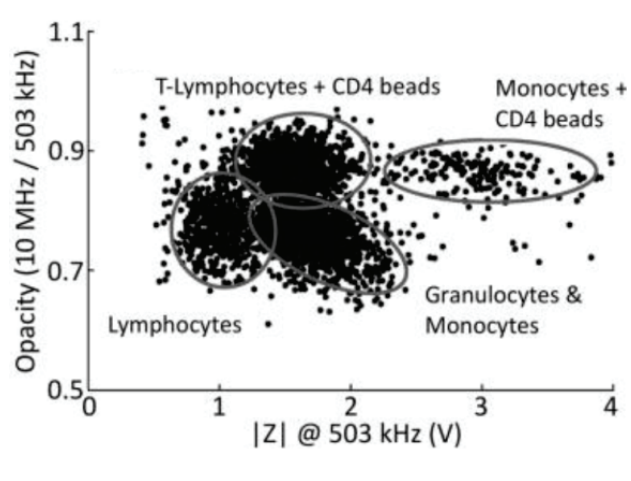

(c)

Figure 6. (a) The first-generation microfluidic impedance and fluorescent flow cytometry where a cell flows between two pairs of electrodes and the optical detection region composed of dual laser excitation, three color detection and dual frequency impedance measurement; (b) Impedance and fluorescent measurement results. Based on low frequency impedance amplitudes, lymphocytes can be differentiated from monocytes and neutrophils due to significantly smaller cell sizes. High frequency impedance amplitudes were used to differentiate neutrophils from monocytes due to significant differences in membrane capacitance. Note that these impedance based classification were validated by the simultaneous fluorescent detection by fluorescently labelling whole blood cells; (c) Whole blood cells mixing with CD4 antibody coated beads were characterized by the microfluidic impedance and fluorescent flow cytometry where lymphocytes, lymphocytes + CD4 beads, granulocytes \& monocytes and monocytes + CD4 beads were successfully classified and confirmed by simultaneous fluorescent characterization $[10,11]$. 

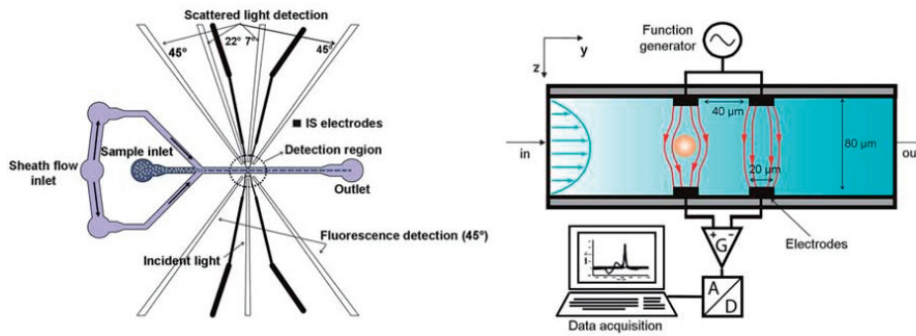

(a)
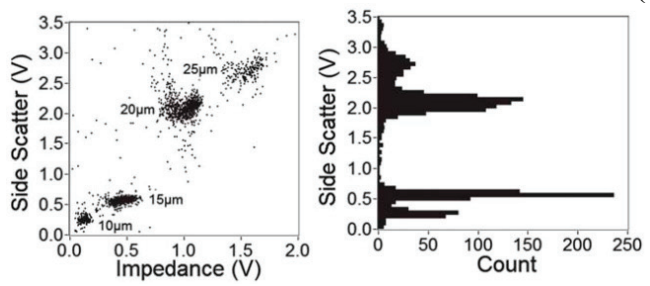

(b)

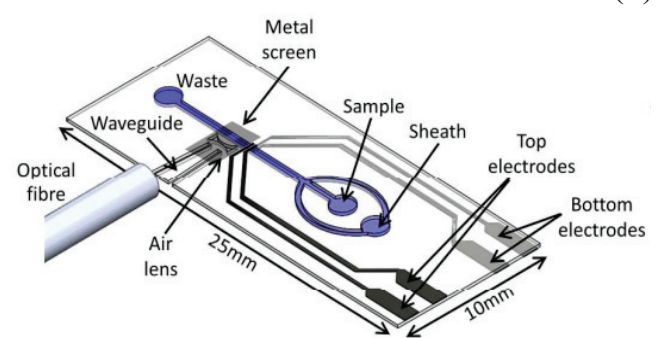

(c)

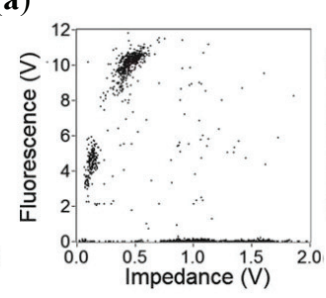

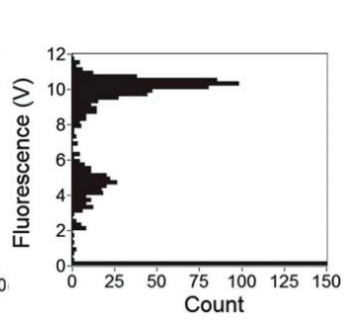

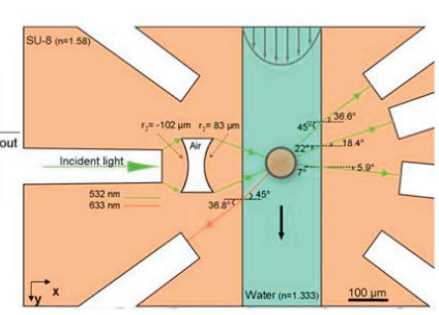

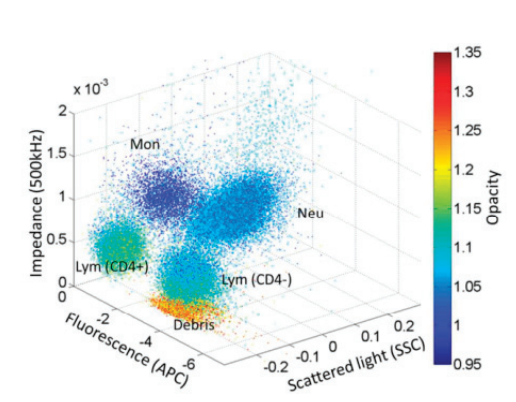

(d)

Figure 7. (a) The second-generation microfluidic impedance and fluorescent flow cytometry with on-chip optical components where a groove in SU-8 holds a fiber to launch incident light, which is then focused into the channel using an air compound lens. Fibers at various angles are used to collect fluorescence emission, optical extinction signal loss, and side scattered light, respectively; (b) Side scattered light, fluorescence and impedance data based classification of a mixture of different beads (10 and $15 \mu \mathrm{m}$ diameter fluorescent, 20 and $25 \mu \mathrm{m}$ diameter plain); (c) A new microfluidic impedance and fluorescent flow cytometry with on-chip waveguides in a sheath-less manner, which can effectively address misalignment of the optical fibers, incident light scatter from multiple interfaces and signal dependent on particle positions; (d) The 3-D scatter plot for CD4 labelled white blood cells based on parameters of side scatter light, fluorescence, and two-frequency impedance data. Both side scattered light and low frequency impedance data provide information on cell sizes, which discriminate smaller lymphocytes from granulocytes. High-frequency impedance data discriminates monocytes from neutrophils due to differences in cell membrane capacitance while CD4 labelled white blood cells were distinguished from white blood cells without CD4 labelling based on fluorescent data $[86,87]$. 


\section{Integrated Point of Care System Based on Microfluidic Impedance Flow Cytometry}

Diagnostic testing at or near the site of patient care is often termed as "near-patient" or "point-of-care" (POC) testing, which can be facilitated by microfluidic technologies [118-123]. Blood cell counting, as the most common clinical indicator of patient health, is one area where microfluidics based POC systems are expected to bring significant advancements [124-126]. Due to the advantages of compactness, low cost and no requirement for optical interfaces, microfluidic impedance flow cytometry has been integrated with sample pretreatment components to enable whole blood cell counting in the POC manner [7,88].

In 2011, Morgan et al. proposed an integrated microfluidic platform based on impedance flow cytometry, enabling the counting of 3-part differential leukocytes (granulocyte, lymphocyte and monocyte), as well as erythrocytes and platelets from raw blood samples [8,88]. As shown in Figure $8 \mathrm{a}$, the integrated system consists of two parts: an impedance detection chip and a microfluidic sample preparation block. The microfluidic sample preparation block performs whole blood loading, pre-treatment and dilution into two separate fluid channels for impedance characterization, respectively. The bottom arm performs analysis of white blood cells with erythrocytes lysed while the upper arm performs counting of red blood cells and platelets.

Figure $8 \mathrm{~b}$ shows the impedance scatter plot of cell membrane opacity (the ratio of impedance measured at 1.7 to $0.5 \mathrm{MHz}$ ) vs. the electrical cell volume (impedance magnitude at $0.5 \mathrm{MHz}$ ) for white blood cells. Consistent with previous studies [10,11], the three main subpopulations (lymphocytes, monocytes and neutrophils) are clearly separated while the top left region represents red blood ghost cells and other debris that are not completely eliminated by the on-chip lysis. Counting of red blood cells and platelets was performed using a single frequency of $0.5 \mathrm{MHz}$, where the cells are easily differentiated by sizes (see Figure 8c). Due to the relatively low number of platelets, platelet concordance was conducted, and the results showed an excellent linearity between the absolute platelet counts obtained from the impedance cytometry system and the hematology analyzer in hospitals.

In 2013, Bashir et al. proposed a microfluidic CD4 ${ }^{+}$and $\mathrm{CD} 8^{+} \mathrm{T}$ Lymphocyte counter for point-of-care HIV diagnostics targeting raw whole blood samples [7,127,128]. As shown in Figure $9 \mathrm{a}$, the integrated microfluidic device is based on differential electrical counting and relies on five on-chip modules that, in sequence, chemically lyses erythrocytes, quenches lysis to preserve leukocytes, enumerates cells electrically, depletes the target cells (CD4 or CD8) with antibodies, and enumerates the remaining cells electrically. Target cell depletion was accomplished through shear stress-based immunocapture, and antibody-coated microposts were used to increase the contact surface areas and enhance the depletion efficiency. Based on the differential electrical counting method, which relies on two-frequency impedance data to classify lymphocytes, monocytes and neutrophils, $\mathrm{CD}^{+}$and $\mathrm{CD}^{+}$cell difference before and after the target cell depletion region was quantified (see Figure $9 b$ ).

Figure 9c,d show $\mathrm{CD}^{+}$and $\mathrm{CD} 8^{+} \mathrm{T}$ cell count results between chip and flow cytometry control with a close match using healthy $(n=18)$ and HIV-infected patient $(n=32)$ blood samples, respectively. By providing accurate cell counts in less than $20 \mathrm{~min}$, this approach can be potentially 
implemented as a handheld, battery-powered instrument that would deliver simple HIV diagnostics to patients anywhere in the world, regardless of geography or socioeconomic status.

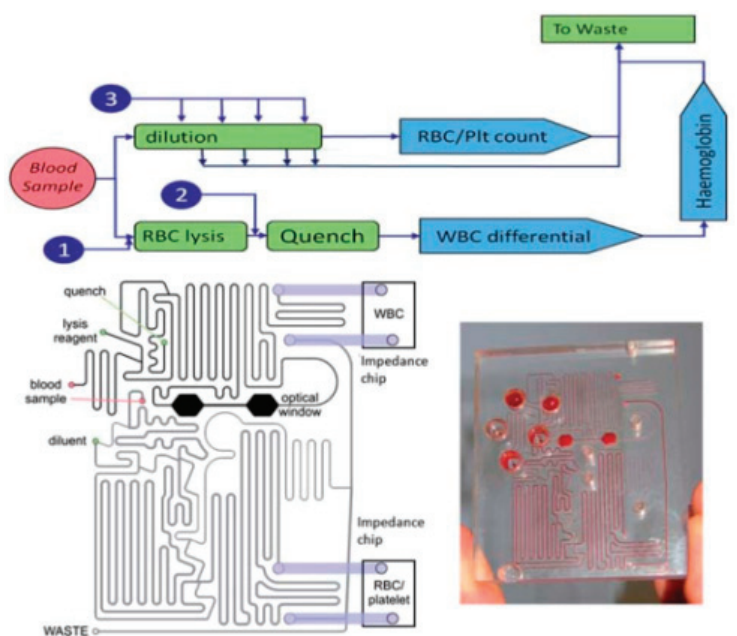

(a)

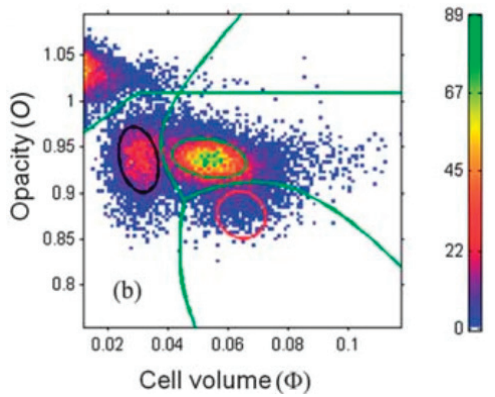

(b)

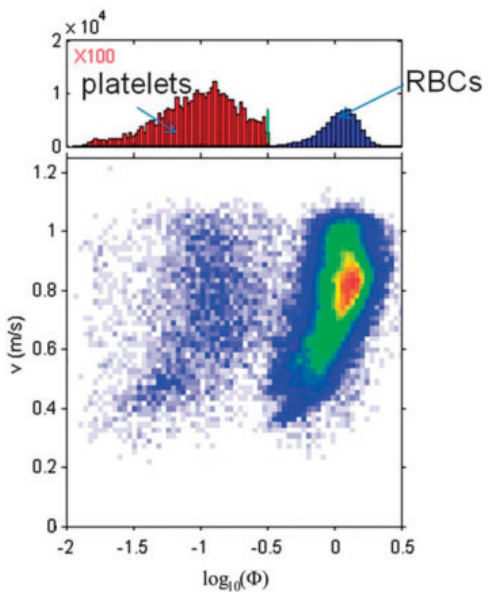

(c)

Figure 8. (a) The integrated point of care system based on microfluidic impedance flow cytometry enabling whole blood cell counting. The integrated system consists of two parts, an impedance measuring chip and a microfluidic sample preparation block. The bottom arm performs analysis of white blood cells with erythrocytes lysed while the upper arm performs counting of red blood cells and platelets; (b) The impedance scatter plot of cell membrane opacity $v s$. the electrical cell volume for classification of three main subpopulations of white blood cells (lymphocytes, monocytes and neutrophils); (c) Counting of red blood cells and platelets was performed based on single-frequency impedance data, where the cells are easily differentiated by sizes [88]. 


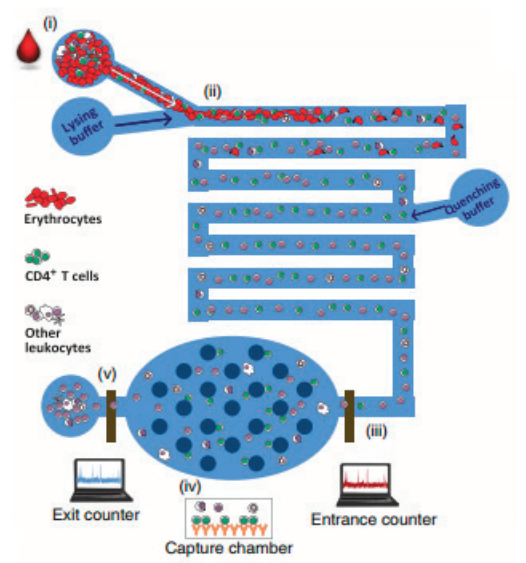

(a)
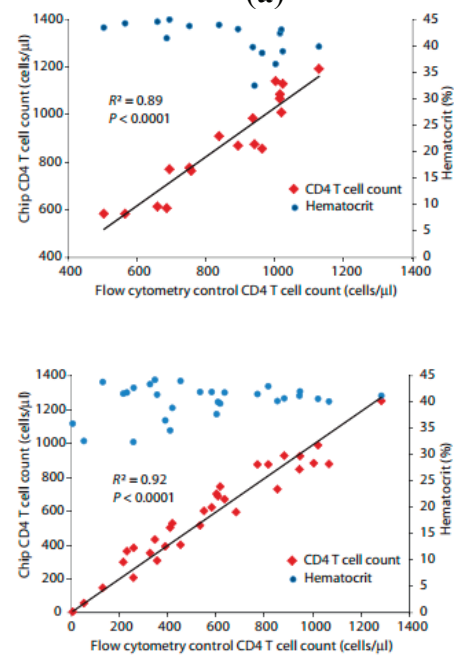

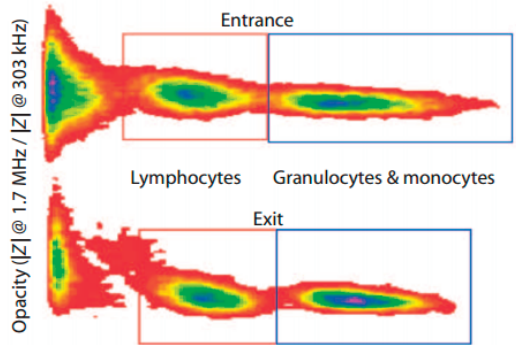

$|z| @ 303$ kHz (V)

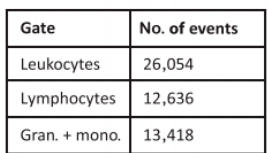

\begin{tabular}{|l|l|}
\hline Gate & No. of events \\
\hline Leukocytes & 10,073 \\
\hline Lymphocytes & 5,614 \\
\hline Gran. + mono. & 4,459 \\
\hline
\end{tabular}

(b)

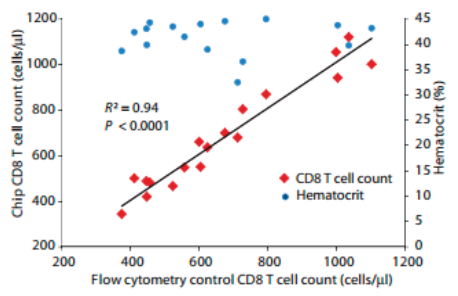

(c)

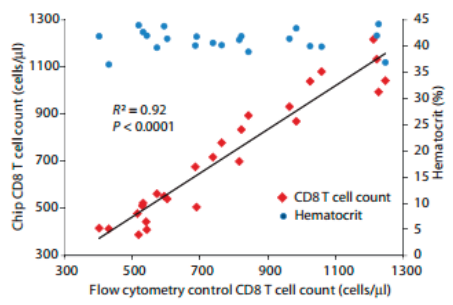

(d)
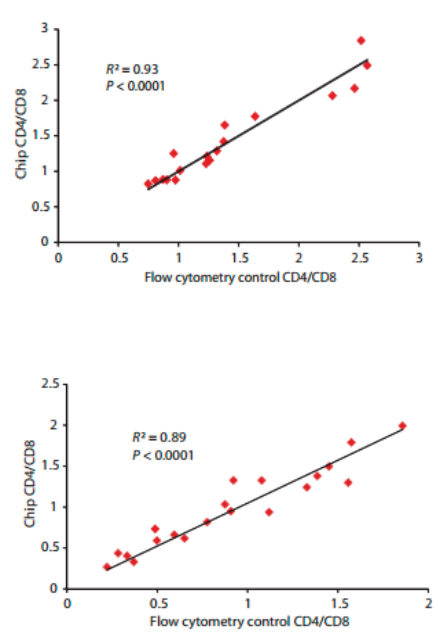

Figure 9. (a) The integrated point of care system based on microfluidic impedance flow cytometry enabling $\mathrm{CD}^{+}$and $\mathrm{CD}^{+} \mathrm{T}$ Lymphocyte counting. The integrated microfluidic device relies on five on-chip modules that are, in sequence, chemically lyses erythrocytes, quenches lysis to preserve leukocytes, enumerates cells electrically, depletes the target cells (CD4 or CD8) with antibodies, and enumerates the remaining cells electrically. Target cell depletion was accomplished through shear stress-based immunocapture; (b) Scatter plots of opacity vs. the low-frequency impedance amplitude for white blood cells before and after CD4 and CD8 depletion; CD4 ${ }^{+}$and $\mathrm{CD}^{+} \mathrm{T}$ cell count results between chip and flow cytometry control with a close match using healthy $(n=18)(\mathbf{c})$ and HIV-infected patient $(n=32)(\mathbf{d})$ blood samples, respectively [7]. 


\section{Conclusions and Outlook}

In this review, recent developments in the field of microfluidic impedance flow cytometry have been discussed from four perspectives: (1) early developments of microfluidic impedance flow cytometry for single-cell electrical property characterization; (2) microfluidic impedance flow cytometry with enhanced sensitivity; (3) microfluidic impedance and optical flow cytometry for single-cell analysis and (4) integrated point of care system based on microfluidic impedance flow cytometry.

From the aspect of technical development, microfluidic impedance flow cytometry enabling high-throughput characterization of size-independent intrinsic cellular electrical properties (e.g., specific membrane capacitance, a throughput of $\sim 1000$ cells per second) should be under intensive research. The majority of reported microfluidic impedance flow cytometry can collect cellular electrical properties in a high-throughput manner, which, however, are only capable of reporting size-dependent electrical properties (e.g., impedance values at several specific frequencies). Although these parameters can indicate membrane capacitance and cytoplasm resistance, they are dependent on cell sizes and specific experimental conditions (e.g., channel geometries and electrode dimensions). Since these parameters do not directly reflect intrinsic cellular electrical properties, it would be difficult to evaluate cellular status and classify cell types based on these parameters.

Recently, impedance spectroscopy and the constriction channel design were combined, enabling the quantification of $\mathrm{C}_{\text {specific membrane }}$ and $\sigma_{\text {cytoplasm from hundreds of cells }}$ [85,103]. In addition, a microfluidic platform was developed where the cross-sectional area of the constriction channel is under regulation, effectively addressing the issue of constriction channel blockage [104]. However, the throughput of such microfluidic devices is roughly one cell per second, which is still low as compared to the conventional flow cytometry ( $\sim 1000$ cells per second). Thus, further technical development should focus on microfluidic impedance flow cytometry enabling high-throughput size-independent intrinsic electrical property characterization of single cells.

From the perspectives of clinical applications, microfluidic impedance flow cytometry can be used to classify tumor cells, stem cells, and blood cells in a label-free manner. In the field of tumor cell classification $[5,64,83]$, paired high- and low-metastatic carcinoma strains and tumor cells as well as their counterparts with single oncogenes under regulation were successfully classified based on cellular electrical properties [5]. Further studies should be conducted to characterize electrical properties of human tumor samples and evaluate the feasibility of tumor classification based on cellular electrical properties.

As to the stem cell classification, undifferentiated and differentiated mouse embryonic carcinoma cells (P19) based on impedance data at $50 \mathrm{kHz}, 250 \mathrm{kHz}, 500 \mathrm{kHz}$ and $1 \mathrm{MHz}$ were differentiated [6] where it was speculated that the capacitance of stem cells can vary as they experience various stages of differentiation. These results provide some preliminary data along this direction but more data are needed for a decisive conclusion. For example, during stem cell differentiation, impedance data should be collected at multiple time points. This can help sketch the trend in how electrical properties of stem cells evolve as they differentiate into adult cells. 
Furthermore, electrical properties of human rather than mouse stem cells should be characterized to further evaluate the possibility of stem cell classification based on cellular electrical properties.

In the field of red blood cell classification based on cellular electrical properties [9,84], in 2013, Chandrakasan et al. developed a microfluidic impedance flow cytometry capable of differentiating $P$. falciparum infected red blood cells from uninfected red blood cells based on amplitude and phase data at $2 \mathrm{MHz}$. However, multiple-frequency impedance data are suggested to further evaluate the electrical properties of various types of red blood cells. For white blood cell differentiation, since it has a close relationship with point of care applications, intensive research efforts have been devoted [7,8,10,11,88] (e.g., CD4 ${ }^{+} \mathrm{T}$ lymphocyte counting [7]). Further studies should compare these approaches with other point of care methods and test a large number of human samples with statistical significance.

\section{Acknowledgments}

The authors would like to acknowledge financial support from National Natural Science Foundation of China (Grant No. 61201077), National Basic Research Program of China (973 Program, Grant No. 2014CB744600), National Natural Science Foundation of China (Grant No. 81261120561 and 61431019), National High Technology Research and Development Program of China (863 Program, Grant No. 2014AA093408), Chang Gung Memorial Hospital in Taiwan (Grant No. CMRPD2C0102 and CMRPD2D0041) and Beijing NOVA Program of Science and Technology. In addition, the authors would like to thank Rong Long for beneficial discussions.

\section{Author Contributions}

Jian Chen reviewed the early developments of microfluidic impedance flow cytometry for single-cell electrical property characterization. Chengcheng Xue and Yang Zhao reviewed the microfluidic impedance flow cytometry with enhanced sensitivity. Deyong Chen and Min-Hsien Wu reviewed microfluidic impedance and optical flow cytometry for single-cell analysis and Junbo Wang reviewed integrated point of care systems based on microfluidic impedance flow cytometry.

\section{Conflicts of Interest}

The authors declare no conflict of interest.

\section{References}

1. Morgan, H.; Sun, T.; Holmes, D.; Gawad, S.; Green, N.G. Single cell dielectric spectroscopy. J. Phys. D-Appl. Phys. 2007, 40, 61-70.

2. Valero, A.; Braschler, T.; Renaud, P. A unified approach to dielectric single cell analysis: Impedance and dielectrophoretic force spectroscopy. Lab Chip 2010, 10, 2216-2225.

3. Liang, X.; Graham, K.A.; Johannessen, A.C.; Costea, D.E.; Labeed, F.H. Human oral cancer cells with increasing tumorigenic abilities exhibit higher effective membrane capacitance. Integr. Biol. 2014, 6, 545-554. 
4. Coley, H.M.; Labeed, F.H.; Thomas, H.; Hughes, M.P. Biophysical characterization of mdr breast cancer cell lines reveals the cytoplasm is critical in determining drug sensitivity. Biochim. Biophys. Acta 2007, 1770, 601-608.

5. Zhao, Y.; Zhao, X.T.; Chen, D.Y.; Luo, Y.N.; Jiang, M.; Wei, C.; Long, R.; Yue, W.T.; Wang, J.B.; Chen, J. Tumor cell characterization and classification based on cellular specific membrane capacitance and cytoplasm conductivity. Biosens. Bioelectron. 2014, 57, 245-253.

6. Song, H.; Wang, Y.; Rosano, J.M.; Prabhakarpandian, B.; Garson, C.; Pant, K.; Lai, E. A microfluidic impedance flow cytometer for identification of differentiation state of stem cells. Lab Chip 2013, 13, 2300-2310.

7. Watkins, N.N.; Hassan, U.; Damhorst, G.; Ni, H.; Vaid, A.; Rodriguez, W.; Bashir, R. Microfluidic CD4+ and CD8+ t lymphocyte counters for point-of-care hiv diagnostics using whole blood. Sci. Trans. Med. 2013, 5, 214 ra170.

8. Han, X.; van Berkel, C.; Gwyer, J.; Capretto, L.; Morgan, H. Microfluidic lysis of human blood for leukocyte analysis using single cell impedance cytometry. Anal. Chem. 2012, 84, 1070-1075.

9. Du, E.; Ha, S.; Diez-Silva, M.; Dao, M.; Suresh, S.; Chandrakasan, A.P. Electric impedance microflow cytometry for characterization of cell disease states. Lab Chip 2013, 13, 3903-3909.

10. Holmes, D.; Pettigrew, D.; Reccius, C.H.; Gwyer, J.D.; van Berkel, C.; Holloway, J.; Davies, D.E.; Morgan, H. Leukocyte analysis and differentiation using high speed microfluidic single cell impedance cytometry. Lab Chip 2009, 9, 2881-2889.

11. Holmes, D.; Morgan, H. Single cell impedance cytometry for identification and counting of CD4 t-cells in human blood using impedance labels. Anal. Chem. 2010, 82, 1455-1461.

12. Zheng, Y.; Nguyen, J.; Wei, Y.; Sun, Y. Recent advances in microfluidic techniques for single-cell biophysical characterization. Lab Chip 2013, 13, 2464-2483.

13. Labeed, F.H.; Coley, H.M.; Thomas, H.; Hughes, M.P. Assessment of multidrug resistance reversal using dielectrophoresis and flow cytometry. Biophys. J. 2003, 85, 2028-2034.

14. Broche, L.M.; Labeed, F.H.; Hughes, M.P. Extraction of dielectric properties of multiple populations from dielectrophoretic collection spectrum data. Phys. Med. Biol. 2005, 50, 2267-2274.

15. Duncan, L.; Shelmerdine, H.; Hughes, M.P.; Coley, H.M.; Hubner, Y.; Labeed, F.H. Dielectrophoretic analysis of changes in cytoplasmic ion levels due to ion channel blocker action reveals underlying differences between drug-sensitive and multidrug-resistant leukaemic cells. Phys. Med. Biol. 2008, 53, N1-N7.

16. Vykoukal, D.M.; Gascoyne, P.R.; Vykoukal, J. Dielectric characterization of complete mononuclear and polymorphonuclear blood cell subpopulations for label-free discrimination. Integr. Biol. 2009, 1, 477-484.

17. Wu, L.; Lanry Yung, L.Y.; Lim, K.M. Dielectrophoretic capture voltage spectrum for measurement of dielectric properties and separation of cancer cells. Biomicrofluidics 2012, 6, 14113.

18. Bebarova, M. Advances in patch clamp technique: Towards higher quality and quantity. Gen. Physiol. Biophys. 2012, 31, 131-140. 
19. Kornreich, B.G. The patch clamp technique: Principles and technical considerations. J. Vet. Cardiol. 2007, 9, 25-37.

20. Dale, T.J.; Townsend, C.; Hollands, E.C.; Trezise, D.J. Population patch clamp electrophysiology: A breakthrough technology for ion channel screening. Mol. Biosyst. 2007, 3, 714-722.

21. Liem, L.K.; Simard, J.M.; Song, Y.; Tewari, K. The patch clamp technique. Neurosurgery 1995, 36, 382-392.

22. Cahalan, M.; Neher, E. Patch clamp techniques: An overview. Methods Enzymol. 1992, 207, 3-14.

23. Sakmann, B.; Neher, E. Patch clamp techniques for studying ionic channels in excitable membranes. Annu. Rev. Physiol. 1984, 46, 455-472.

24. Auerbach, A.; Sachs, F. Patch clamp studies of single ionic channels. Annu. Rev. Biophys. Bioeng. 1984, 13, 269-302.

25. Rohani, A.; Varhue, W.; Su, Y.H.; Swami, N.S. Electrical tweezer for highly parallelized electrorotation measurements over a wide frequency bandwidth. Electrophoresis 2014, 35, 1795-1802.

26. Lei, U.; Sun, P.H.; Pethig, R. Refinement of the theory for extracting cell dielectric properties from dielectrophoresis and electrorotation experiments. Biomicrofluidics 2011, 5, 044109.

27. Voyer, D.; Frenea-Robin, M.; Buret, F.; Nicolas, L. Improvements in the extraction of cell electric properties from their electrorotation spectrum. Bioelectrochemistry 2010, 79, 25-30.

28. Jones, T.B. Basic theory of dielectrophoresis and electrorotation. IEEE Eng. Med. Biol. Mag. 2003, 22, 33-42.

29. Goater, A.D.; Pethig, R. Electrorotation and dielectrophoresis. Parasitology 1998, 117 (Suppl.), S177-S189.

30. Fuhr, G.; Glaser, R.; Hagedorn, R. Rotation of dielectrics in a rotating electric high-frequency field-model experiments and theoretical explanation of the rotation effect of living cells. Biophys. J. 1986, 49, 395-402.

31. Egger, M.; Donath, E.; Ziemer, S.; Glaser, R. Electrorotation--a new method for investigating membrane events during thrombocyte activation. Influence of drugs and osmotic pressure. Biochim. Biophys. Acta 1986, 861, 122-130.

32. Fuhr, G.; Hagedorn, R.; Goring, H. Separation of different cell-types by rotating electric-fields. Plant Cell Physiol. 1985, 26, 1527-1531.

33. Yobas, L. Microsystems for cell-based electrophysiology. J. Micromech. Microeng. 2013, 23, 083002.

34. Sabuncu, A.C.; Zhuang, J.; Kolb, J.F.; Beskok, A. Microfluidic impedance spectroscopy as a tool for quantitative biology and biotechnology. Biomicrofluidics 2012, 6, 34103.

35. Sun, T.; Morgan, H. Single-cell microfluidic impedance cytometry: A review. Microfluid. Nanofluid. 2010, 8, 423-443.

36. Cheung, K.C.; di Berardino, M.; Schade-Kampmann, G.; Hebeisen, M.; Pierzchalski, A.; Bocsi, J.; Mittag, A.; Tarnok, A. Microfluidic impedance-based flow cytometry. Cytom. A 2010, 77, 648-666. 
37. Wootton, R.C.; Demello, A.J. Microfluidics: Exploiting elephants in the room. Nature 2010, 464, 839-840.

38. Whitesides, G.M. The origins and the future of microfluidics. Nature 2006, 442, 368-373.

39. Squires, T.M.; Quake, S.R. Microfluidics: Fluid physics at the nanoliter scale. Rev. Mod. Phys. 2005, 77, 977.

40. Sackmann, E.K.; Fulton, A.L.; Beebe, D.J. The present and future role of microfluidics in biomedical research. Nature 2014, 507, 181-189.

41. El-Ali, J.; Sorger, P.K.; Jensen, K.F. Cells on chips. Nature 2006, 442, 403-411.

42. Meyvantsson, I.; Beebe, D.J. Cell culture models in microfluidic systems. Annu. Rev. Anal. Chem. 2008, 1, 423-449.

43. Paguirigan, A.L.; Beebe, D.J. Microfluidics meet cell biology: Bridging the gap by validation and application of microscale techniques for cell biological assays. BioEssays News Rev. Mol. Cell. Dev. Biol. 2008, 30, 811-821.

44. Velve-Casquillas, G.; Le Berre, M.; Piel, M.; Tran, P.T. Microfluidic tools for cell biological research. Nano Today 2010, 5, 28-47.

45. Thompson, A.M.; Paguirigan, A.L.; Kreutz, J.E.; Radich, J.P.; Chiu, D.T. Microfluidics for single-cell genetic analysis. Lab Chip 2014, 14, 3135-3142.

46. Yin, H.; Marshall, D. Microfluidics for single cell analysis. Curr. Opin. Biotechnol. 2012, 23, 110-119.

47. Lecault, V.; White, A.K.; Singhal, A.; Hansen, C.L. Microfluidic single cell analysis: From promise to practice. Curr. Opin. Chem. Biol. 2012, 16, 381-390.

48. Ryan, D.; Ren, K.; Wu, H. Single-cell assays. Biomicrofluidics 2011, 5, 21501.

49. Zare, R.N.; Kim, S. Microfluidic platforms for single-cell analysis. Annu. Rev. Biomed. Eng. 2010, 12, 187-201.

50. Sims, C.E.; Allbritton, N.L. Analysis of single mammalian cells on-chip. Lab Chip 2007, 7, 423-440.

51. Di Carlo, D.; Lee, L.P. Dynamic single-cell analysis for quantitative biology. Anal. Chem. 2006, 78, 7918-7925.

52. Cho, Y.H.; Yamamoto, T.; Sakai, Y.; Fujii, T.; Kim, B. Development of microfluidic device for electrical/physical characterization of single cell. J. Microelectromech. Syst. 2006, $15,287-295$.

53. Jang, L.S.; Wang, M.H. Microfluidic device for cell capture and impedance measurement. Biomed. Microdevices 2007, 9, 737-743.

54. Senez, V.; Lennon, E.; Ostrovidov, S.; Yamamoto, T.; Fujita, H.; Sakai, Y.; Fujii, T. Integrated 3-d silicon electrodes for electrochemical sensing in microfluidic environments: Application to single-cell characterization. IEEE Sens. J. 2008, 8, 548-557.

55. Hua, S.Z.; Pennell, T. A microfluidic chip for real-time studies of the volume of single cells. Lab Chip 2009, 9, 251-256.

56. Wang, M.H.; Jang, L.S. A systematic investigation into the electrical properties of single hela cells via impedance measurements and comsol simulations. Biosens. Bioelectron. 2009, 24, 2830-2835. 
57. Jang, L.S.; Li, H.H.; Jao, J.Y.; Chen, M.K.; Liu, C.F. Design and fabrication of microfluidic devices integrated with an open-ended mems probe for single-cell impedance measurement. Microfluid. Nanofluid. 2010, 8, 509-519.

58. Malleo, D.; Nevill, J.T.; Lee, L.P.; Morgan, H. Continuous differential impedance spectroscopy of single cells. Microfluid. Nanofluid. 2010, 9, 191-198.

59. Han, K.H.; Han, A.; Frazier, A.B. Microsystems for isolation and electrophysiological analysis of breast cancer cells from blood. Biosens. Bioelectron. 2006, 21, 1907-1914.

60. Cho, S.B.; Thielecke, H. Micro hole-based cell chip with impedance spectroscopy. Biosens. Bioelectron. 2007, 22, 1764-1768.

61. James, C.D.; Reuel, N.; Lee, E.S.; Davalos, R.V.; Mani, S.S.; Carroll-Portillo, A.; Rebeil, R.; Martino, A.; Apblett, C.A. Impedimetric and optical interrogation of single cells in a microfluidic device for real-time viability and chemical response assessment. Biosens. Bioelectron. 2008, 23, 845-851.

62. Cho, Y.; Kim, H.S.; Frazier, A.B.; Chen, Z.G.; Shin, D.M.; Han, A. Whole-cell impedance analysis for highly and poorly metastatic cancer cells. J. Microelectromech. Syst. 2009, 18, 808-817.

63. Chen, J.; Zheng, Y.; Tan, Q.; Zhang, Y.L.; Li, J.; Geddie, W.R.; Jewett, M.A.; Sun, Y. A microfluidic device for simultaneous electrical and mechanical measurements on single cells. Biomicrofluidics 2011, 5, 14113.

64. Kang, G.; Kim, Y.-J.; Moon, H.-S.; Lee, J.-W.; Yoo, T.-K.; Park, K.; Lee, J.-H. Discrimination between the human prostate normal cell and cancer cell by using a novel electrical impedance spectroscopy controlling the cross-sectional area of a microfluidic channel. Biomicrofluidics 2013, 7, 044126.

65. Tan, Q.; Ferrier, G.A.; Chen, B.K.; Wang, C.; Sun, Y. Quantification of the specific membrane capacitance of single cells using a microfluidic device and impedance spectroscopy measurement. Biomicrofluidics 2012, 6, 034112.

66. Jang, L.S.; Huang, P.H.; Lan, K.C. Single-cell trapping utilizing negative dielectrophoretic quadrupole and microwell electrodes. Biosens. Bioelectron. 2009, 24, 3637-3644.

67. Lan, K.C.; Jang, L.S. Integration of single-cell trapping and impedance measurement utilizing microwell electrodes. Biosens. Bioelectron. 2011, 26, 2025-2031.

68. Hong, J.-L.; Lan, K.-C.; Jang, L.-S. Electrical characteristics analysis of various cancer cells using a microfluidic device based on single-cell impedance measurement. Sens. Actuators $B$ Chem. 2012, 173, 927-934.

69. Chen, N.-C.; Chen, C.-H.; Chen, M.-K.; Jang, L.-S.; Wang, M.-H. Single-cell trapping and impedance measurement utilizing dielectrophoresis in a parallel-plate microfluidic device. Sens. Actuators B Chem. 2014, 190, 570-577.

70. Thein, M.; Asphahani, F.; Cheng, A.; Buckmaster, R.; Zhang, M.Q.; Xu, J. Response characteristics of single-cell impedance sensors employed with surface-modified microelectrodes. Biosens. Bioelectron. 2010, 25, 1963-1969. 
71. Asphahani, F.; Wang, K.; Thein, M.; Veiseh, O.; Yung, S.; Xu, J.A.; Zhang, M.Q. Single-cell bioelectrical impedance platform for monitoring cellular response to drug treatment. Phys. Biol. 2011, 8, 015006.

72. Asphahani, F.; Zheng, X.H.; Veiseh, O.; Thein, M.; Xu, J.; Ohuchi, F.; Zhang, M.Q. Effects of electrode surface modification with chlorotoxin on patterning single glioma cells. Phys. Chem. Chem. Phys. 2011, 13, 8953-8960.

73. Wu, Y.; Han, X.; Benson, J.D.; Almasri, M. Micromachined coulter counter for dynamic impedance study of time sensitive cells. Biomed. Microdevices 2012, 14, 739-750.

74. Wu, Y.; Benson, J.D.; Critser, J.K.; Almasri, M. Note: Microelectromechanical systems coulter counter for cell monitoring and counting. Rev. Sci. Instrum. 2010, 81, 076103.

75. Swanton, E.M.; Curby, W.A.; Lind, H.E. Experiences with the coulter counter in bacteriology. Appl. Microbiol. 1962, 10, 480-485.

76. Bryan, A.K.; Engler, A.; Gulati, A.; Manalis, S.R. Continuous and long-term volume measurements with a commercial coulter counter. PLOS ONE 2012, 7, e29866.

77. Gawad, S.; Schild, L.; Renaud, P. Micromachined impedance spectroscopy flow cytometer for cell analysis and particle sizing. Lab Chip 2001, 1, 76-82.

78. Gou, H.-L.; Zhang, X.-B.; Bao, N.; Xu, J.-J.; Xia, X.-H.; Chen, H.-Y. Label-free electrical discrimination of cells at normal, apoptotic and necrotic status with a microfluidic device. J. Chromatogr. A 2011, 1218, 5725-5729.

79. Cheung, K.; Gawad, S.; Renaud, P. Impedance spectroscopy flow cytometry: On-chip label-free cell differentiation. Cytom. Part A 2005, 65A, 124-132.

80. Haandbaek, N.; Burgel, S.C.; Heer, F.; Hierlemann, A. Characterization of subcellular morphology of single yeast cells using high frequency microfluidic impedance cytometer. Lab Chip 2014, 14, 369-377.

81. Bernabini, C.; Holmes, D.; Morgan, H. Micro-impedance cytometry for detection and analysis of micron-sized particles and bacteria. Lab Chip 2011, 11, 407-412.

82. Haandbaek, N.; With, O.; Burgel, S.C.; Heer, F.; Hierlemann, A. Resonance-enhanced microfluidic impedance cytometer for detection of single bacteria. Lab Chip 2014, 14, 3313-3324.

83. Chen, J.; Zheng, Y.; Tan, Q.; Shojaei-Baghini, E.; Zhang, Y.L.; Li, J.; Prasad, P.; You, L.; Wu, X.Y.; Sun, Y. Classification of cell types using a microfluidic device for mechanical and electrical measurement on single cells. Lab Chip 2011, 11, 3174-3181.

84. Zheng, Y.; Shojaei-Baghini, E.; Azad, A.; Wang, C.; Sun, Y. High-throughput biophysical measurement of human red blood cells. Lab Chip 2012, 12, 2560-2567.

85. Zhao, Y.; Chen, D.; Li, H.; Luo, Y.; Deng, B.; Huang, S.B.; Chiu, T.K.; Wu, M.H.; Long, R.; $\mathrm{Hu}, \mathrm{H}$; et al. A microfluidic system enabling continuous characterization of specific membrane capacitance and cytoplasm conductivity of single cells in suspension. Biosens. Bioelectron. 2013, 43C, 304-307.

86. Barat, D.; Spencer, D.; Benazzi, G.; Mowlem, M.C.; Morgan, H. Simultaneous high speed optical and impedance analysis of single particles with a microfluidic cytometer. Lab Chip 2012, 12, 118-126. 
87. Spencer, D.C.; Elliott, G.; Morgan, H. A sheath-less combined optical and impedance micro-cytometer. Lab Chip 2014, 14, 3064-3073.

88. Van Berkel, C.; Gwyer, J.D.; Deane, S.; Green, N.G.; Holloway, J.; Hollis, V.; Morgan, H. Integrated systems for rapid point of care (poc) blood cell analysis. Lab Chip 2011, 11, 1249-1255.

89. Gawad, S.; Cheung, K.; Seger, U.; Bertsch, A.; Renaud, P. Dielectric spectroscopy in a micromachined flow cytometer: Theoretical and practical considerations. Lab Chip 2004, 4, 241-251.

90. Mernier, G.; Piacentini, N.; Tornay, R.; Buffi, N.; Renaud, P. Cell viability assessment by flow cytometry using yeast as cell model. Sens. Actuators B Chem. 2011, 154, 160-163.

91. Meissner, R.; Joris, P.; Eker, B.; Bertsch, A.; Renaud, P. A microfluidic-based frequency-multiplexing impedance sensor (fmis). Lab Chip 2012, 12, 2712-2718.

92. Mernier, G.; Hasenkamp, W.; Piacentini, N.; Renaud, P. Multiple-frequency impedance measurements in continuous flow for automated evaluation of yeast cell lysis. Sens. Actuators B Chem. 2012, 170, 2-6.

93. Shaker, M.; Colella, L.; Caselli, F.; Bisegna, P.; Renaud, P. An impedance-based flow microcytometer for single cell morphology discrimination. Lab Chip 2014, 2014, 2548-2555.

94. Choi, H.; Jeon, C.S.; Hwang, I.; Ko, J.; Lee, S.; Choo, J.; Boo, J.-H.; Kim, H.C.; Chung, T.D. A flow cytometry-based submicron-sized bacterial detection system using a movable virtual wall. Lab Chip 2014, 14, 2327-2333.

95. Spencer, D.; Morgan, H. Positional dependence of particles in microfludic impedance cytometry. Lab Chip 2011, 11, 1234-1239.

96. Shelby, J.P.; White, J.; Ganesan, K.; Rathod, P.K.; Chiu, D.T. A microfluidic model for single-cell capillary obstruction by plasmodium falciparum infected erythrocytes. Proc. Natl. Acad. Sci. USA 2003, 100, 14618-14622.

97. Guo, Q.; Reiling, S.J.; Rohrbach, P.; Ma, H. Microfluidic biomechanical assay for red blood cells parasitized by plasmodium falciparum. Lab Chip 2012, 12, 1143-1150.

98. Kwan, J.M.; Guo, Q.; Kyluik-Price, D.L.; Ma, H.; Scott, M.D. Microfluidic analysis of cellular deformability of normal and oxidatively damaged red blood cells. Am. J. Hematol. 2013, 88, 682-689.

99. Rosenbluth, M.J.; Lam, W.A.; Fletcher, D.A. Analyzing cell mechanics in hematologic diseases with microfluidic biophysical flow cytometry. Lab Chip 2008, 8, 1062-1070.

100. Hou, H.W.; Li, Q.S.; Lee, G.Y.H.; Kumar, A.P.; Ong, C.N.; Lim, C.T. Deformability study of breast cancer cells using microfluidics. Biomed. Microdevices 2009, 11, 557-564.

101. Byun, S.; Son, S.; Amodei, D.; Cermak, N.; Shaw, J.; Kang, J.H.; Hecht, V.C.; Winslow, M.M.; Jacks, T.; Mallick, P.; et al. Characterizing deformability and surface friction of cancer cells. Proc. Natl. Acad. Sci. USA 2013, 110, 7580-7585.

102. Luo, Y.N.; Chen, D.Y.; Zhao, Y.; Wei, C.; Zhao, X.T.; Yue, W.T.; Long, R.; Wang, J.B.; Chen, J. A constriction channel based microfluidic system enabling continuous characterization of cellular instantaneous young's modulus. Sens. Actuators B Chem. 2014, 202, 1183-1189. 
103. Zhao, Y.; Chen, D.; Luo, Y.; Li, H.; Deng, B.; Huang, S.-B.; Chiu, T.-K.; Wu, M.-H.; Long, R.; Hu, H.; et al. A microfluidic system for cell type classification based on cellular size-independent electrical properties. Lab Chip 2013, 13, 2272-2277.

104. Huang, S.B.; Zhao, Z.; Chen, D.Y.; Lee, H.C.; Luo, Y.N.; Chiu, T.K.; Wang, J.B.; Chen, J.; $\mathrm{Wu}$, M.H. A clogging-free microfluidic platform with an incorporated pneumatically-driven membrane-based active valve enabling specific membrane capacitance and cytoplasm conductivity characterization of single cells. Sens. Actuators B Chem. 2014, 190, 928-936.

105. Morgan, H.; Holmes, D.; Green, N.G. High speed simultaneous single particle impedance and fluorescence analysis on a chip. Curr. Appl. Phys. 2006, 6, 367-370.

106. Benazzi, G.; Holmes, D.; Sun, T.; Mowlem, M.C.; Morgan, H. Discrimination and analysis of phytoplankton using a microfluidic cytometer. Iet Nanobiotechnol. 2007, 1, 94-101.

107. Gawad, S.; Sun, T.; Green, N.G.; Morgan, H. Impedance spectroscopy using maximum length sequences: Application to single cell analysis. Rev. Sci. Instrum. 2007, 78, 054301.

108. Holmes, D.; She, J.K.; Roach, P.L.; Morgan, H. Bead-based immunoassays using a micro-chip flow cytometer. Lab Chip 2007, 7, 1048-1056.

109. Sun, T.; Gawad, S.; Bernabini, C.; Green, N.G.; Morgan, H. Broadband single cell impedance spectroscopy using maximum length sequences: Theoretical analysis and practical considerations. Meas. Sci. Technol. 2007, 18, 2859-2868.

110. Sun, T.; Green, N.G.; Gawad, S.; Morgan, H. Analytical electric field and sensitivity analysis for two microfluidic impedance cytometer designs. Iet Nanobiotechnol. 2007, 1, 69-79.

111. Sun, T.; Holmes, D.; Gawad, S.; Green, N.G.; Morgan, H. High speed multi-frequency impedance analysis of single particles in a microfluidic cytometer using maximum length sequences. Lab Chip 2007, 7, 1034-1040.

112. Sun, T.; van Berkel, C.; Green, N.G.; Morgan, H. Digital signal processing methods for impedance microfluidic cytometry. Microfluid. Nanofluid. 2009, 6, 179-187.

113. Barat, D.; Benazzi, G.; Mowlem, M.C.; Ruano, J.M.; Morgan, H. Design, simulation and characterisation of integrated optics for a microfabricated flow cytometer. Opt. Commun. 2010, 283, 1987-1992.

114. Gawad, S.; Holmes, D.; Benazzi, G.; Renaud, P.; Morgan, H. Impedance spectroscopy and optical analysis of single biological cells and organisms in microsystems. Methods Mol. Biol. 2010, 583, 149-182.

115. Sun, T.; Bernabini, C.; Morgan, H. Single-colloidal particle impedance spectroscopy: Complete equivalent circuit analysis of polyelectrolyte microcapsules. Langmuir 2010, 26, 3821-3828.

116. Sun, T.; Swindle, E.J.; Collins, J.E.; Holloway, J.A.; Davies, D.E.; Morgan, H. On-chip epithelial barrier function assays using electrical impedance spectroscopy. Lab Chip 2010, 10, 1611-1617.

117. Sun, T.; Tsuda, S.; Zauner, K.P.; Morgan, H. On-chip electrical impedance tomography for imaging biological cells. Biosens. Bioelectron. 2010, 25, 1109-1115. 
118. Kumar, S.; Kumar, S.; Ali, M.A.; Anand, P.; Agrawal, V.V.; John, R.; Maji, S.; Malhotra, B.D. Microfluidic-integrated biosensors: Prospects for point-of-care diagnostics. Biotechnol. J. 2013, 8, 1267-1279.

119. Chin, C.D.; Linder, V.; Sia, S.K. Commercialization of microfluidic point-of-care diagnostic devices. Lab Chip 2012, 12, 2118-2134.

120. Kiechle, F.L.; Holland, C.A. Point-of-care testing and molecular diagnostics: Miniaturization required. Clin. Lab. Med. 2009, 29, 555-560.

121. Sorger, P.K. Microfluidics closes in on point-of-care assays. Nat. Biotechnol. 2008, 26, 1345-1346.

122. Sia, S.K.; Kricka, L.J. Microfluidics and point-of-care testing. Lab Chip 2008, 8, 1982-1983.

123. Linder, V. Microfluidics at the crossroad with point-of-care diagnostics. Analyst 2007, 132, 1186-1192.

124. Yu, Z.T.; Aw Yong, K.M.; Fu, J. Microfluidic blood cell sorting: Now and beyond. Small 2014, 10, 1687-1703.

125. van der Meer, A.D.; Poot, A.A.; Duits, M.H.; Feijen, J.; Vermes, I. Microfluidic technology in vascular research. J. Biomed. Biotechnol. 2009, 2009, 823148.

126. Toner, M.; Irimia, D. Blood-on-a-chip. Annu. Rev. Biomed. Eng. 2005, 7, 77-103.

127. Hassan, U.; Bashir, R. Electrical cell counting process characterization in a microfluidic impedance cytometer. Biomed. Microdevices 2014, 16, 697-704.

128. Hassan, U.; Watkins, N.N.; Edwards, C.; Bashir, R. Flow metering characterization within an electrical cell counting microfluidic device. Lab Chip 2014, 14, 1469-1476. 


\title{
Detecting Antigen-Specific T Cell Responses: From Bulk Populations to Single Cells
}

\section{Chansavath Phetsouphanh, John James Zaunders and Anthony Dominic Kelleher}

\begin{abstract}
A new generation of sensitive T cell-based assays facilitates the direct quantitation and characterization of antigen-specific $T$ cell responses. Single-cell analyses have focused on measuring the quality and breadth of a response. Accumulating data from these studies demonstrate that there is considerable, previously-unrecognized, heterogeneity. Standard assays, such as the ICS, are often insufficient for characterization of rare subsets of cells. Enhanced flow cytometry with imaging capabilities enables the determination of cell morphology, as well as the spatial localization of the protein molecules within a single cell. Advances in both microfluidics and digital PCR have improved the efficiency of single-cell sorting and allowed multiplexed gene detection at the single-cell level. Delving further into the transcriptome of single-cells using RNA-seq is likely to reveal the fine-specificity of cellular events such as alternative splicing (i.e., splice variants) and allele-specific expression, and will also define the roles of new genes. Finally, detailed analysis of clonally related antigen-specific T cells using single-cell TCR RNA-seq will provide information on pathways of differentiation of memory $\mathrm{T}$ cells. With these state of the art technologies the transcriptomics and genomics of Ag-specific T cells can be more definitively elucidated.
\end{abstract}

Reprinted from Int. J. Mol. Sci. Cite as: Phetsouphanh, C.; Zaunders, J.J.; Kelleher, A.D. Detecting Antigen-Specific T Cell Responses: From Bulk Populations to Single Cells. Int. J. Mol. Sci. 2015, 16, 18878-18893.

\section{Introduction}

A wide variety of assays can be used to characterize $T$ cell responses in order to determine the state and capability of the immune system. Such studies can reveal fundamental mechanisms underlying immunity aid the design of clinical diagnostics, help develop intervention therapies, and determine signatures of effective immune responses [1]. Primarily, research on antigen (Ag)-specific CD4+ T cells has been done using bulk-sorted populations by focusing on small sets of cells defined by selected markers that were hypothesized to identify homogenous sub-populations; for example, central versus effector memory cells defined by surface markers such as CD45RA/RO isoforms and CCR7 or CD62L expression [2-4]. However, accumulating data demonstrate that there is considerable heterogeneity within the Ag-specific population on the basis of genomic differences, cytokine secretion profiles, function, and trafficking markers [1,5-8].

Standard analytical technologies historically have measured the average response from highly heterogeneous populations. Common assays that detected cell proliferation, cytolytic activity, and cytokine expression have yielded valuable insights into disease pathogenesis and immunity to microbes such as viruses and tumour or self-antigens. However, these assays examined multiple parameters at the population level, where the implicit averaging of many measurements may mask the specific involvement of individual cells and the interactions that can occur between neighbouring 
cells. These technologies made it difficult to infer the characteristics of rare subsets of cells, such as Ag-specific T cell responses, without first purifying subsets of T cells. Even when purified, the T cell subsets were generally identified on the basis of a relatively small number of markers, compared to the much larger number of cell surface proteins expressed by T cells [9-11]. Single cell analyses are beginning to show that these approaches have underestimated heterogeneity.

Recently, single-cell analyses have focused on measuring the quality and breadth of a response. Variations in the expression of molecules between individual cells are thought to play an important role in functionally diversifying an immune response at the population level and also determining the diverse anatomical locations of individual cells. Advances in genome-wide quantitative analysis of single cells can provide an important vehicle that allows the investigator to make further insights into the variation between individual cells and to determine how these impact on the fine specificity of the nature and regulation of the immune response.

The challenge of understanding heterogeneity between cells, particularly tumour cells [12-14] has driven many of the major technological advances, resulting in the design of powerful instruments, protocols, and analysis protocols that enable the elucidation of DNA, RNA, and protein expression at the single-cell level. Flow cytometry has been widely adopted as the cornerstone of highthroughput analysis of specific protein expression and phosphorylation states of single cells within complex populations. Cell sorting has typically been used to purify up to six populations at a time from these mixtures of cells. The recent coupling of this technology with microfluidics and genomewide deep sequencing at the single cell level has enabled further insights into cell biology. Singlecell genomics provides the basis for unbiased investigations into the molecular and functional consequences of cellular variability. In this review, the advantages and disadvantages of standard T cell response detection assays will be discussed. Newer technologies to more comprehensively define $\mathrm{T}$ cell responses at the single-cell level will be examined and the advances in single-cell genomics will be highlighted.

\section{Standard Assays Are Insufficient for the Detection of T Cell Responses at the Single Cell Level}

Measuring T lymphocyte proliferation after antigenic or mitogenic stimulation is an important parameter used in diagnosis of various immuno-deficiencies and in the monitoring a variety of immune responses. Measurement of the incorporation of tritiated thymidine $\left[{ }^{3} \mathrm{H}\right]$ into lymphocyte DNA is a common approach used to determine the extent of antigen- or mitogen-driven cell proliferation [15]. Disadvantages of this assay include: The response of individual populations cells cannot be delineated without cell sorting; the inherent variability of the assay; the limitations and safety of handling radioactive material; and the labour-intensive nature of the protocols (i.e., multiple wash steps involved, PBMC isolation, and inability to determine which cells are proliferating) [16].

More recent approaches that overcome some of these problems include the use of the cytoplasmic dyes, such as carboxyfluorescein succinimidyl ester (CFSE), to track lymphocyte proliferation [17]. CFSE covalently binds to amino groups on intracellular macromolecules that anchor the dye. CFSE is inherited equally by daughter cells after division, resulting in the halving of mean fluorescence with each generation. Disadvantages of this assay are: The time required for proliferation detection is usually 
4-6 days; and high concentrations can cause cell toxicity and impair expression of activation markers $[17,18]$. However cell subpopulations can be tracked if markers are expressed stably. The use of multi-parameter flow cytometry allows other information about the proliferating cells and their phenotype to be accumulated. Further, the stability, or otherwise, of markers during the process of proliferation in response to antigen can be definitively determined.

ELISPOT (enzyme-linked immunospot) and intracellular cytokine staining (ICS) are widely used assays for the detection and enumeration of antigen-specific $\mathrm{T}$ cells. ELISPOT assays detect antigen-induced secretion of cytokines (usually IFN- $\gamma$ ) trapped by an immobilized antibody on a nitrocellulose membrane and visualized by an enzyme-coupled secondary antibody. ICS also detects cytokines produced by antigen-stimulated $\mathrm{T}$ cells, via the detection of the cytokines, such as IFN- $\gamma$, TNF, and IL-2, trapped within golgi/ER bodies in the cytoplasm through inactivation of granule secretion by brefeldin A or monensin, and visualized via fluorochrome-labelled monoclonal antibodies $(\mathrm{mAb})$ after cell permeabilisation followed by flow cytometry.

The use of both assays is associated with implicit a priori assumptions regarding the importance of certain cytokines in the responses of interest (i.e., IFN- $\gamma$ ) and do not take into account the other aspects of the $\mathrm{T}$ cell response; most often used to measure Th1 or Th2 responses, but can also be used to measure effector molecules such as Granzyme B. ELISPOT can only measure one or two parameters at a time and, without depletion or purification of cell subsets, the source of the cytokine cannot be determined. Multiple parameters in the ICS need to be optimized for each model system, such as stimulation, fixation/permeabilization, and appropriate controls need to be considered [19]. These assays are typically restricted to Th1 subset identification, as Th2 cytokine detection works poorly in these assays, and Th17 responses are usually only detectable when mitogens are used. This is due to a combination of factors including limitations in availability of $\mathrm{mAb}$ and the lower levels of cytokine production. Further, these assays do not allow live isolation of cells for downstream functional or molecular analysis and bulk populations with high cell numbers are required for each assay [20,21].

Detailed information of responding single cells in ELISPOT assays is limited to the level of markers used to purify cells prior to the assay, while ICS assays are limited by the number of additional fluorescence markers that can added to the assay. Each assay comes with issues of assay validation and quality control, with advantages and disadvantages depending on the nature of the responses detected. For example, standardization of assay requires the removal of differences in confounding variables when comparing experiments performed by the same or different users under the same conditions. Issues, such as choice of starting material, coating techniques, incubation and washing steps, and personal preference in reading spot development, need to be taken into account [22]. Additionally, currently-available flow cytometric analysis is generally limited to a maximum of 16 fluorochromes, but the use of up to 29 parameters has been recently reported $[1,23,24]$. The use of $m A$ bs labeled with rare-earth isotopes, combining flow cytometric and mass cytometric technologies $\left(\mathrm{CyTOF}^{\circledR}\right)$, also greatly increases the number of parameters able to be defined on each cell $[25,26]$. A caveat with the $\mathrm{CyTOF}^{\circledR}$ platform is that it still lacks the sensitivity of standard fluorescences cytometry, up to three-fold less sensitive and requires more cells for staining. 
Nevertheless, the available number of parameters still does not approach the number of proteins that are variably expressed by T cells [27,28].

\subsection{Using MHC-Multimers to Identify Antigen-Specific T Cells}

Recombinant multimeric complexes of soluble recombinant MHC molecules often referred to as "tetramers" have emerged as a key tool for elucidation of the frequency of antigen-specific T cells in vitro, particularly in viral infections and post-vaccinations. Since 1996, there has been a revolution in the characterization of antigen-specific $\mathrm{T}$ cells, due to the development of reagents consisting of soluble multimerized MHC-I peptide complexes to detect epitope specific CD8+ T cells using flow cytometry through their increased avidity for TCR [29,30]. MHC-I tetramers combined with other staining techniques have been used to examine detailed information about antigen specific $\mathrm{T}$ cells, such as levels of activation, effector function, proliferation, and apoptosis [31], but importantly because they bind to the surface of T cells based only on their expression of TCR which recognize the incorporated epitope cells which are anergic, "stunned" or "exhausted" can be identified by this technology [32-36]. Further, because only surface staining is required, the cells can be sorted and used for functional assays and/or RNA profiling. This has led to the colossal expansion of information on antigen-specific CD8 $+\mathrm{T}$ cells and to a lesser extent antigen-specific CD4+ $\mathrm{T}$ cells, because of the more limited availability of MHC-II tetramers [37].

MHC-I-peptide complexes consist of an invariant light chain ( $\beta_{2}$-microglobulin), a polymorphic heavy chain and a specific $8-10$ amino acid peptide. The presence of a cognate peptide in the antigen-binding groove is essential for the formation of MHC-I molecules. This association is based on specific complimentary interactions between amino acid side chains at the anchor positions of the peptide and allele-specific pockets [38]. This helps ensure that the recombinant MHC-I heterodimers that refold are conformationally correct. Typically the formation of these soluble MHC-I multimers is dependent upon the specific biotinylation of a tail engineered to replace the transmembrane and cytoplasmic domains of the heavy chain. The biotinylated heterodimers are then bound to each of the four binding sites of fluorescently labeled streptavidin, forming a tetrameric complex. However, other strategies for multimeriziation have been employed to achieve the necessary increase in avidity between MHC-I and TCR to allow interactions stable enough for robust staining of $\mathrm{T}$ cells and subsequent identification by flow cytometry [39-42]. A possible drawback from this technique is that individual tetramers have to be designed for each epitope of interest, and then are only applicable to individuals carrying a particular class I allele. The shelf life of the constructs are variable, and positive and negative controls need to be carefully identified to ensure staining is specific, thus making the process very labour intensive, especially if multiple epitope-specific responses are to be studied simultaneously [37]. To overcome these limitations, dextramers (multimers with a dextran backbone) were developed, each dextramer molecule bears multiple fluorescein and streptavidin components. These dextramers were able to identify low frequency antigen-specific $\mathrm{T}$ cells and produced stronger signals than their tetramer counterpart [43].

In contrast to the relative ease of production MHC class I tetramers, the development of MHC class II tetramers has been more difficult. There are multiple reasons for this. Firstly, the peptide-binding grove depends on correct association of variable alpha and beta chains making the 
synthesis of some alleles inefficient. The characteristics determining binding of antigenic peptide to MHC-II are different to MHC-I in several ways. Secondly, the stability of the class II molecule is not dependent on the binding of peptide in the groove. The groove is open-ended at either end allowing longer peptides to bind and these may bind in different registers within the groove. Further, efficient loading of peptide occurs at acid $\mathrm{pH}$, is dependent upon molecules such as HLA-DM and DO catalysing the efficient exchange of peptide for the CLIP peptide and the peptide receptive state of the class II molecule is rapidly lost. These conditions are difficult to reproduce in vitro. In addition, there is much greater promiscuity of peptide binding to class II than class I, which has resulted in much lower rates of definitive description of class II restricted epitopes [44,45]. Many methods have been explored in an attempt solve these problems. One of these methods is producing class II proteins by co-expressing the $\alpha$ and $\beta$ subunits in mammalian or insect cells and relying on in vivo, rather than in vitro, re-folding, as is the case with proteins produced from E. coli [44]. Unfortunately, much of the material bound to the binding groove is not the epitope of interest and the exchange of peptide into the binding groove is often inefficient. To overcome this constructs expressing a fusion protein, consisting of peptide fused to MHC-II $\beta$ N-terminus via a flexible linker region have been trialled. This covalently attached peptide has preferential access to the peptide-binding site, thus increasing the rate of the correct peptide occupying the binding groove $[46,47]$. Other modifications used to enhance the assembly of the subunits have included the use of leucine zippers and chimeric IgG Fc domains to promote assembly and stability of the heterodimer $[48,49]$. Combined these approaches have resulted in a slow, but steady, increase in the availability of the pool of reliable MHC-II multimers, though their availability is still far more limited than MHC-I equivalents. With these methods of MHC-II production, it is now theoretically possible to identify a subset of antigen-specific CD4+ T cells with multimeric complexes [37,50]. This will facilitate the isolation of single antigen-specific CD4+ T cells for downstream analysis [51,52]. MHC/peptide tetramers, termed streptamers, were developed for this purpose. Streptamers can be reversibly dissociated from binding to antigen-specific CD4+ T cells, this feature allows these cells to remain functionally active, contrary to conventional tetramers that impairs lytic function and proliferation of the bound cells [53].

\subsection{Cell Surface Detection of Antigen-Specific T Cells}

Apart from ICS and multimeric MHC-II complexes, other approaches have been reported to use flow cytometry to identify Ag-specific T cells, including cell surface trapping of cytokines using magnetic bead technology (Miltenyi cytokine-capture system ${ }^{\circledR}$ ) [54], or the use of activation-induced trafficking of some intracellular markers to the cell surface such as CD40L expressed on CD4+ T cells $[55,56]$. However, both these assays are limited by their detection of only one effector molecule at a time and we know that many important responses, such as Tregs and CTL, are not detected by either of these methods. 


\subsection{CD25/OX40 Assay}

CD4+ T cell responses are pivotal to the regulation of the immune system during viral infection. Due in great part to the difficulties associated with the synthesis and use of class II multimers and therefore an inability to identify and isolate epitope specific CD4+ T cells, our understanding of the molecular basis of CD4+ function has fallen behind that of CD8+ T cells. Thus an assay that allows live isolation of antigen specific $\mathrm{T}$ cells is necessary.

A recent flow cytometric assay, developed by Zaunders et al., employs the co-expression of CD25 (IL-2R $\alpha$ ) and CD134/OX40 (a TNF receptor family member) to identify antigen-specific CD4+ T cells. The co-expression of these two molecules in CD4+ T cells is very low in peripheral blood. However, with antigenic stimulation in vitro, their expression becomes up regulated over time, peaking at approximately $44 \mathrm{~h}$ post-stimulation. Advantages of this assay are that it detects a more global population of antigen-specific cells that appear to include all Th1, Th2, Th17, Tfh, and Treg lineages and is, therefore, not biased by detection of a particular cytokine or effector molecule, resulting in higher levels of antigen-specific T cells than ICC and ELISPOT. Further, it allows live isolation of individual cells for downstream functional and molecular characterization [7,57-62].

\section{Enhanced Flow Cytometry Techniques for Single-Cell Analysis}

Flow cytometry has been extensively used to analyse protein expression within cells. The relatively recent development combining microscopic imaging and fluorescence-activated flow cytometry has allowed a more in-depth examination of single cells. Each cell can be examined for morphology, as well as the spatial localization of the protein molecules within the cell. Instruments, such as the Imagestream ${ }^{\circledR}$, allow rapid acquisition and processing, enabling measurements of thousands of cells per second, which is an advantage over conventional microscopy platforms [63]. This combination technology has been useful for the determination of protein localization, cell morphology, observation of protein interactions during signalling cascades and cellular uptake of foreign particles [64,65]. Although highly useful for providing information on single cells, the measurements acquired are only obtained at a single snapshot in time.

Newer technologies that complement and augment the data generated from flow cytometry have been developed. These technologies can assess the functional and transcriptional dynamics of cells at the microliter to picolitre scale. The two types of techniques that have emerged as tools to detect immune responses are microfluidic systems and spatial arrays with nanolitre-scale wells. For a detailed review on nanolitre micro-well and micro-dense arrays please refer to a review by Lindstrom, et al. [66].

Micro-dense arrays contain up to 100,000 sub-nanolitre welled compartments that allow the isolation or distribution of single-cells and measurement of cellular function, protein secretion and mRNA in parallel. This platform provides the ability to examine cellular interaction between different cell types and provides highly-specific resolution not found when using bulk populations. These cells can be deposited into nanowells for co-culture by dispensing each cell onto the array sequentially and allowing cells to settle via gravity, these cells can then be used for further analyses [1]. Co-cultures of cells (e.g., one effector cell and one antigen-presenting cell) in individual 
wells can provide insight into intercellular signaling and interaction dynamics. This technology can be used to assess antigen-specific interactions between cognate $\mathrm{T}$ cells and their corresponding antigen presenting cell to detect activation, cytolysis and cytokine production [67]. This approach has demonstrated that HIV-specific CD8+ T cells responses initially involve either cytolysis or secretion of pro-inflammatory cytokines (i.e., IFN $\gamma$ ) $[68,69]$.

Nanolitre micro-well arrays enable the study of single-cell phenotyping of rare cells, such as antigen-specific T cells and B cells. Studies on these rare subsets have revealed structure-function relationships between molecular synapses and signalling cascades [70]. Co-culture studies together with barcoded antibody arrays enabled the examination of the influence of paracrine signalling molecules on tumour cell function and signalling networks via the multiplexed detection of both intracellular and secreted proteins/cytokines [71,72]. These nano-arrays have also been used to examine serial killing capacities of Natural Killer (NK) cells, whereby NK cells were able to kill MHC-I deficient tumour cells and also showed that simultaneous interaction with several target cells increases the cytotoxic responsiveness of NK cells [73]. This platform has been used to show to the extent of variability among homogeneous populations. Dura et al. investigated the heterogeneity of CD8 T cells upon antigen presentation and correlated this with early activation events. They discovered that the cells showed relatively homogeneous calcium mobilization with high antigen stimuli with uniform timings of activation. However, the response pattern became more heterogeneous with lower antigen concentrations. These cell populations could be grouped into distinct clusters. Measurement of early signalling events simultaneously revealed high heterogeneity in ERK phosphorylation in single-cells, despite uniform timing and stimulus strength [74]. This study demonstrates the potential of micro-scale tools to clarify the complex intercellular interactions initiating and regulating $\mathrm{T}$ cell activation through the measurement of multiple parameters over a substantial number of individual cells. However, there are some technical limitations in this system, which include the limited control on the fluidic microenvironment required to maintain the cells in culture, as well as the risk of crosscontamination between wells during the rinsing of the array chips [75].

\section{Microfluidics and Digital PCR at the Single-Cell Level}

Reverse transcription quantitative polymerase chain reaction (RT-PCR) has been extensively used to examine gene expression patterns in $\mathrm{T}$ cells and has been the basis for many systems biology approaches profiling cellular activity. It provides exceptional specificity and sensitivity and has been adapted for the measurement of gene expression in single cells [7,76]. The challenges of single-cell RT-PCR studies include the cumbersome and laborious steps in purifying mRNA from individual cells, and the difficulty in synthesizing and purifying cDNA from single cells [77]. These difficulties arise from the loss of starting material during cell isolation, lysis, and cDNA synthesis steps. The loss of material may be caused by mRNA degradation, adhesion to plastics, and inefficient reverse transcription [78,79]. Microfluidic devices have been developed as platforms to overcome these particular problems and to handle the low reaction volumes required for single-cell analyses, while allowing relatively high-throughput, whereby multiple samples can be run simultaneously on the same device under standardized conditions. 
One of the commercially-available valve-based microfluidic qPCR systems that have been successfully used for single cell studies is the Dynamic Array ${ }^{\mathrm{TM}}$ (Fluidigm). This is a low-volume (nanolitre) system that allows high-throughput with assessment of up to 96 parameters in 96 cells per run. It allows low copy detection while being used for large-scale studies [76]. The concordance of copy number detection between microfluidics and digital PCR compared to conventional PCR has been demonstrated [80]. This technology offers a higher level of precision and can be used to measure rare transcripts [81], as well as small RNA species (i.e., microRNA) [82,83].

Mingueneau et al. used this platform to examine early thymocyte differentiation of $\alpha \beta \mathrm{T}$ cells. Gene expression patterns during early $\mathrm{T}$ cell differentiation were measured. It was found that during transit through the $\mathrm{CD} 4+\mathrm{CD} 8+$ stage, these double-positive thymocytes showed a global repression of housekeeping genes, which is rare among other cells of the immune system and correlated highly with the expression of c-Myc. They also identified genetic signatures that distinguished cells destined for positive selection versus apoptosis [84]. Johnson et al. used the Fluidigm ${ }^{\circledR}$ platform to define transcriptional profiles of HIV-specific CD4 $+\mathrm{T}$ cells. In doing so they identified a distinct transcriptional signature of $\mathrm{HIV}$-specific cytolytic CD4+ T cells compared to Th1 cells, and these signatures were similar to features found in HIV-specific CD8+ T cells. These cytolytic CD4+ T cells also showed comparable killing activity to their CD8 counterpart and worked co-operatively to destroy virally infected target cells [85].

These examples demonstrate the innovative uses of these technologies; however, only a selected number of genes can be examine within one cell at a given time. Although this technology expands on the fairly outdated capabilities of traditional PCR, the elucidation of the entire transcriptome would be too cumbersome for this platform. Separation of single cells using microfluidics and the extraction of total DNA or RNA for next-generation sequencing would solve this issue. An example of this is the Fluidigm ${ }^{\circledR} \mathrm{C} 1$ single-cell isolation instrument which allows automated cell capture for RNA/DNA extraction and nucleic acid amplification that is highly useful for RNA-seq and downstream genomic analysis. Microtools such as these allow clinical samples consisting of very small cell numbers to be examined [86]. Examination of cells from cytobrushes and biopsies allows the comparison between cell phenotypes, cell-to-cell communication [87], and function at mucosal sites, which is important in many disease conditions, such as HIV-1 infection.

\section{Single-Cell Genomics Analysis via Next-Generation Deep Sequencing}

A new and important extension of bulk transcription technologies, single-cell RNA sequencing (RNA-seq) has emerged as a powerful tool for mRNA expression analysis and allows genome-wide transcriptomics to be explored [88]. The transcriptome encompasses an essential part of cell identity and function, as RNA is essential for regulation, house-keeping, effector and messenger roles. The state of a specific single-cell (e.g., antigen-specific T cells) can be assessed via the profiling of all coding and non-coding transcripts that eventually leads to genome-wide transcriptomics analysis. RNA-seq techniques involve the conversion of cellular RNA transcripts into cDNA and subsequent sequencing in parallel by using next-generation sequencing technologies [89]. This technology enables high-resolution analysis of single-cells, whereby important cellular events such as alternative 
splicing (i.e., splice variants) and allele-specific expression can be studied, that will also aid the discovery of new genes [90].

Single-cell RNA sequencing has been to investigate the role of de novo hormone synthesis in T helper cells and its role in T cell homeostasis. Mahata et al. demonstrated that that Th2 cells produce the steroid, pregnenolone, which inhibits $\mathrm{T}$ helper cell proliferation and B cell class switching. They proposed that this lympho-steroid is produced in an intrinsic manner by Th2 cells during allergic immune responses to restore immune homeostasis [91]. Shalek et al. used single-cell transcriptomics to assess heterogeneity of mouse bone marrow-derived dendritic cells (BMDCs) responding to lipopolysaccharides. They found bimodal variance in the RNA abundance and splicing patterns within responding cells. The observed splicing patterns displayed high levels of heterogeneity between cells. They identified 137 highly variable, but co-regulated, antiviral response genes that may be propagated through an interferon feedback loop involving the transcription factors Stat 2 and Irf7 [89]. These studies highlight the promise and power of single-cell RNA-seq. This technology can be used to uncover the functional diversity between cells, as well as, discovering new gene regulation circuits.

\subsection{Detecting Clonally-Related Ag-Specific T Cells Using TCR RNA-seq}

A single-cell method to assess $\mathrm{T}$ cell receptor beta chain (TRBV) and alpha chain (TRAV) sequence data of sorted CD4+ and CD8 $+\mathrm{T}$ cells has recently been published [92]. This method also describes a method allowing simultaneous measurement of mRNA transcripts for up to $34 \mathrm{CD} 4+\mathrm{T}$ cell lineage-defining transcription factors and cytokines. Three steps of nested PCR amplification from a single cell, including barcoding from each well of up to 20 separate 96 -well plates, followed by pooling and deep sequencing, allows high throughput TCR sequencing and RNA profiling in parallel in $1000 \mathrm{~s}$ of single cells, which will dramatically increase our ability to consider relationships between TCR repertoire and T cell phenotypic or functional subsets in a range of immune responses. Previous approaches using cloning and Sanger sequencing have not been feasible for this number of cells. Also, the combination with other genomic data on the transcription factors and cytokines, using this approach is an extremely favourable combination of cost-effectiveness and high throughput, for the first time definitively matching information on clonality and genotype. Detailed analysis of clonally-related antigen-specific $\mathrm{T}$ cells will finally provide information on pathways of differentiation of memory $\mathrm{T}$ cells not possible by other currently available techniques.

\section{Conclusions}

The final goal of complete genomics of the full range of single antigen-specific T cells is getting closer, due to very substantial and rapid improvements in identification of antigen-specific $\mathrm{T}$ cells, particularly CD4 $+\mathrm{T}$ cells, combined with advances in single cell mRNA technology (Figure 1). Finally, it is hoped that such studies will discriminate favourable outcomes after vaccination, or after pathogenic infection and provide a road map for rationale, rather than empiric development of vaccines and immunotherapeutics for chronic infections and drug resistant cancers. 

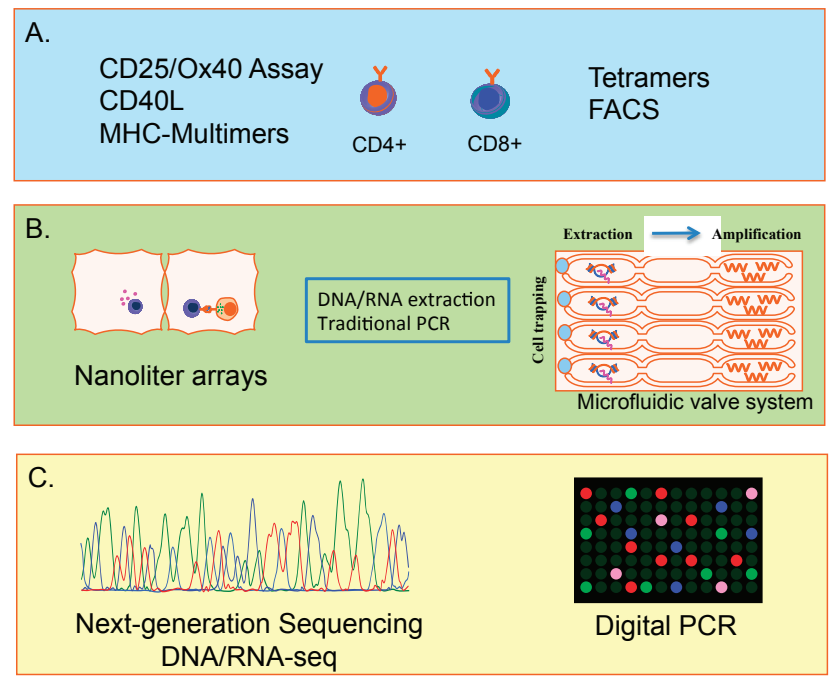

Figure 1. Analysis antigen-specific T cells at the single cell level pipeline. (A) Detection of antigen-specific CD4+ and CD8+ T cells using multiple assays and MHC-multimers; (B) Functional analysis of single Ag-specific cells via micro-well dense arrays and nanolitre arrays or single cell trapping using microfluidic valves for further molecular extraction/amplification; (C) Transcriptomic analysis using digital PCR and next-generation sequencing technologies (i.e., RNA-seq) (coloured lines and dots represent fluorescence signals).

\section{Acknowledgments}

Authors would like to thank the NHMRC (National Health and Medical Research Council) (Canberra, Australia) for grant funding.

\section{Author Contributions}

Chansavath Phetsouphanh wrote manuscript; John James Zaunders and Anthony Dominic Kelleher edited and finessed manuscript for publication.

\section{Conflicts of Interest}

The authors declare no conflict of interest.

\section{References}

1. Chattopadhyay, P.K.; Gierahn, T.M.; Roederer, M.; Love, J.C. Single-cell technologies for monitoring immune systems. Nat. Immunol. 2014, 15, 128-135.

2. Sallusto, F.; Lanzavecchia, A. Heterogeneity of CD4+ memory T cells: Functional modules for tailored immunity. Eur. J. Immunol. 2009, 39, 2076-2082. 
3. Sallusto, F.; Lenig, D.; Forster, R.; Lipp, M.; Lanzavecchia, A. Two subsets of memory T lymphocytes with distinct homing potentials and effector functions. Nature 1999, 401, 708-712.

4. Sallusto, F.; Geginat, J.; Lanzavecchia, A. Central memory and effector memory T cell subsets: Function, generation, and maintenance. Annu. Rev. Immunol. 2004, 22, 745-763.

5. Li, H.; Margolick, J.B.; Bream, J.H.; Nilles, T.L.; Langan, S.; Bui, H.T.; Sylwester, A.W.; Picker, L.J.; Leng, S.X. Heterogeneity of CD4+ and CD8+ T-cell responses to cytomegalovirus in HIV-infected and HIV-uninfected men who have sex with men. J. Infect. Dis. 2014, 210, 400-404.

6. Becattini, S.; Latorre, D.; Mele, F.; Foglierini, M.; de Gregorio, C.; Cassotta, A.; Fernandez, B.; Kelderman, S.; Schumacher, T.N.; Corti, D.; et al. T cell immunity. Functional heterogeneity of human memory $\mathrm{CD}^{+} \mathrm{T}$ cell clones primed by pathogens or vaccines. Science 2015, 347, 400-406.

7. Phetsouphanh, C.; Xu, Y.; Amin, J.; Seddiki, N.; Procopio, F.; Sekaly, R.P.; Zaunders, J.J.; Kelleher, A.D. Characterization of transcription factor phenotypes within antigen-specific CD4+ T cells using qualitative multiplex single-cell RT-PCR. PLOS ONE 2013, 8, e 74946.

8. Zaunders, J.; Jing, J.; Leipold, M.; Maecker, H.; Kelleher, A.D.; Koch, I. Computationally efficient multidimensional analysis of complex flow cytometry data using second order polynomial histograms. Cytometry A 2015, doi:10.1002/cyto.a.22704.

9. Evans, E.J.; Hene, L.; Sparks, L.M.; Dong, T.; Retiere, C.; Fennelly, J.A.; Manso-Sancho, R.; Powell, J.; Braud, V.M.; Rowland-Jones, S.L.; et al. The T cell surface-how well do we know it? Immunity 2003, 19, 213-223.

10. McMichael, A.J.; Ogg, G.; Wilson, J.; Callan, M.; Hambleton, S.; Appay, V.; Kelleher, T.; Rowland-Jones, S. Memory CD8+ T cells in HIV infection. Philos. Trans. R. Soc. Lond. B Biol. Sci. 2000, 355, 363-367.

11. McMichael, A.J.; Callan, M.; Appay, V.; Hanke, T.; Ogg, G.; Rowland-Jones, S. The dynamics of the cellular immune response to HIV infection: Implications for vaccination. Philos. Trans. R. Soc. Lond. B Biol. Sci. 2000, 355, 1007-1011.

12. Chen, Y.C.; Allen, S.G.; Ingram, P.N.; Buckanovich, R.; Merajver, S.D.; Yoon, E. Single-cell Migration Chip for Chemotaxis-based Microfluidic Selection of Heterogeneous Cell Populations. Sci. Rep. 2015, 5, 9980.

13. Li, S.; Zhu, X.; Liu, B.; Wang, G.; Ao, P. Endogenous molecular network reveals two mechanisms of heterogeneity within gastric cancer. Oncotarget 2015, 6, 13607-13627.

14. Norton, K.A.; Popel, A.S.; Pandey, N.B. Heterogeneity of chemokine cell-surface receptor expression in triple-negative breast cancer. Am. J. Cancer Res. 2015. 5, 1295-1307.

15. Cavanagh, B.L.; Walker, T.; Norazit, A.; Meedeniya, A.C. Thymidine analogues for tracking DNA synthesis. Molecules 2011, 16, 7980-7993.

16. Last'ovicka, J.; Budinsky, V.; Spisek, R.; Bartunkova, J. Assessment of lymphocyte proliferation: CFSE kills dividing cells and modulates expression of activation markers. Cell Immunol. 2009, 256, 79-85.

17. Parish, C.R. Fluorescent dyes for lymphocyte migration and proliferation studies. Immunol. Cell Biol. 1999, 77, 499-508. 
18. De Clerck, L.S.; Bridts, C.H.; Mertens, A.M.; Moens, M.M.; Stevens, W.J. Use of fluorescent dyes in the determination of adherence of human leucocytes to endothelial cells and the effect of fluorochromes on cellular function. J. Immunol. Methods 1994, 172, 115-124.

19. Karlsson, A.C.; Martin, J.N.; Younger, S.R.; Bredt, B.M.; Epling, L.; Ronquillo, R.; Varma, A.; Deeks, S.G.; McCune, J.M.; Nixon, D.F.; et al. Comparison of the ELISPOT and cytokine flow cytometry assays for the enumeration of antigen-specific T cells. J. Immunol. Methods 2003, 283, 141-153.

20. Pala, P.; Hussell, T.; Openshaw, P.J.M. Flow cytometric measurement of intracellular cytokines. J. Immunol. Methods 2000, 243, 107-124.

21. Streeck, H.; Frahm, N.; Walker, B.D. The role of IFN-gamma Elispot assay in HIV vaccine research. Nat. Protoc. 2009, 4, 461-469.

22. Janetzki, S.; Britten, C.M. The impact of harmonization on ELISPOT assay performance. Methods Mol. Biol. 2012, 792, 25-36.

23. Bendall, S.C.; Nolan, G.P.; Roederer, M.; Chattopadhyay, P.K. A deep profiler's guide to cytometry. Trends Immunol. 2012, 33, 323-332.

24. Chattopadhyay, P.K.; Roederer, M. A mine is a terrible thing to waste: High content, single cell technologies for comprehensive immune analysis. Am. J. Transplant. 2015, 15, 1155-1161.

25. Behbehani, G.K.; Bendall, S.C.; Clutter, M.R.; Fantl, W.J.; Nolan, G.P. Single-cell mass cytometry adapted to measurements of the cell cycle. Cytometry A 2012, 81, 552-566.

26. Nolan, G.P. Flow cytometry in the post fluorescence era. Best Pract. Res. Clin. Haematol. 2011, 24, 505-508.

27. Bendall, S.C.; Simonds, E.F.; Qiu, P.; Amir, E.-A.D.; Krutzik, P.O.; Finck, R.; Bruggner, R.V.; Melamed, R.; Trejo, A.; Ornatsky, O.I.; et al. Single-cell mass cytometry of differential immune and drug responses across a human hematopoietic continuum. Science 2011, 332, 687-696.

28. Blonder, J.; Issaq, H.J.; Veenstra, T.D. Proteomic biomarker discovery: It's more than just mass spectrometry. Electrophoresis 2011, 32, 1541-1548.

29. Altman, J.D.; Moss, P.A.; Goulder, P.J.; Barouch, D.H.; McHeyzer-Williams, M.G.; Bell, J.I.; McMichael, A.J.; Davis, M.M. Phenotypic analysis of antigen-specific T lymphocytes. Science 1996, 274, 94-96.

30. McHeyzer-Williams, M.G.; Altman, J.D.; Davis, M.M. Enumeration and characterization of memory cells in the TH compartment. Immunol. Rev. 1996, 150, 5-21.

31. Kurtulus, S.; Hildeman, D. Assessment of CD4(+) and CD8 (+) T cell responses using MHC class I and II tetramers. Methods Mol. Biol. 2013, 979, 71-79.

32. Yi, J.S.; Cox, M.A.; Zajac, A.J. T-cell exhaustion: Characteristics, causes and conversion. Immunology 2010, 129, 474-481.

33. Zajac, A.J.; Blattman, J.N.; Murali-Krishna, K.; Sourdive, D.J.; Suresh, M.; Altman, J.D.; Ahmed, R. Viral immune evasion due to persistence of activated T cells without effector function. J. Exp. Med. 1998, 188, 2205-2213.

34. Appay, V.; Rowland-Jones, S.L. The assessment of antigen-specific CD8+ T cells through the combination of MHC class I tetramer and intracellular staining. J. Immunol. Methods 2002, 268, 9-19. 
35. Appay, V.; Almeida, J.R.; Sauce, D.; Autran, B.; Papagno, L. Accelerated immune senescence and HIV-1 infection. Exp. Gerontol. 2007, 42, 432-437.

36. Huygens, A.; Lecomte, S.; Tackoen, M.; Olislagers, V.; Delmarcelle, Y.; Burny, W.; van Rysselberge, M.; Liesnard, C.; Larsen, M.; Appay, V.; et al. Functional Exhaustion Limits CD4+ and CD8+ T-Cell Responses to Congenital Cytomegalovirus Infection. J. Infect. Dis. 2015, 212, 484-494.

37. Sims, S.; Willberg, C.; Klenerman, P. MHC-peptide tetramers for the analysis of antigen-specific T cells. Expert Rev. Vaccines 2010, 9, 765-774.

38. Leisner, C.; Loeth, N.; Lamberth, K.; Justesen, S.; Sylvester-Hvid, C.; Schmidt, E.G.; Claesson, M.; Buus, S.; Stryhn, A. One-pot, mix-and-read peptide-MHC tetramers. PLoS ONE 2008, 3, e1678.

39. Bakker, A.H.; Schumacher, T.N. MHC multimer technology: Current status and future prospects. Curr. Opin. Immunol. 2005, 17, 428-433.

40. Knabel, M.; Franz, T.J.; Schiemann, M.; Wulf, A.; Villmow, B.; Schmidt, B.; Bernhard, H.; Wagner, H.; Busch, D.H. Reversible MHC multimer staining for functional isolation of T-cell populations and effective adoptive transfer. Nat. Med. 2002, 8, 631-637.

41. Bakker, A.H.; Hoppes, R.; Linnemann, C.; Toebes, M.; Rodenko, B.; Berkers, C.R.; Hadrup, S.R.; van Esch, W.J.; Heemskerk, M.H.; Ovaa, H.; et al. Conditional MHC class I ligands and peptide exchange technology for the human MHC gene products HLA-A1, -A3, -A11, and -B7. Proc. Natl. Acad. Sci. USA 2008, 105, 3825-3830.

42. Hadrup, S.R.; Toebes, M.; Rodenko, B.; Bakker, A.H.; Egan, D.A.; Ovaa, H.; Schumacher, T.N. High-throughput T-cell epitope discovery through MHC peptide exchange. Methods Mol. Biol. 2009, 524, 383-405.

43. Batard, P.; Peterson, D.A.; Devevre, E.; Guillaume, P.; Cerottini, J.C.; Rimoldi, D.; Speiser, D.E.; Winther, L.; Romero, P. Dextramers: New generation of fluorescent MHC class $\mathrm{I} /$ peptide multimers for visualization of antigen-specific CD8+ T cells. J. Immunol. Methods 2006, 310, 136-148.

44. Cameron, T.O.; Norris, P.J.; Patel, A.; Moulon, C.; Rosenberg, E.S.; Mellins, E.D.; Wedderburn, L.R.; Stern, L.J. Labeling antigen-specific CD4(+) T cells with class II MHC oligomers. J. Immunol. Methods 2002, 268, 51-69.

45. Crawford, F.; Kozono, H.; White, J.; Marrack, P.; Kappler, J. Detection of antigen-specific T cells with multivalent soluble class II MHC covalent peptide complexes. Immunity 1998, 8, 675-682.

46. Kozono, H.; White, J.; Clements, J.; Marrack, P.; Kappler, J. Production of soluble MHC class II proteins with covalently bound single peptides. Nature 1994, 369, 151-154.

47. Cunliffe, S.L.; Wyer, J.R.; Sutton, J.K.; Lucas, M.; Harcourt, G.; Klenerman, P.; McMichael, A.J.; Kelleher, A.D. Optimization of peptide linker length in production of MHC class II/peptide tetrameric complexes increases yield and stability, and allows identification of antigen-specific CD4+T cells in peripheral blood mononuclear cells. Eur. J. Immunol. 2002, 32, 3366-3375. 
48. Lebowitz, M.S.; O’Herrin, S.M.; Hamad, A.R.; Fahmy, T.; Marguet, D.; Barnes, N.C.; Pardoll, D.; Bieler, J.G.; Schneck, J.P. Soluble, high-affinity dimers of T-cell receptors and class II major histocompatibility complexes: Biochemical probes for analysis and modulation of immune responses. Cell Immunol. 1999, 192, 175-184.

49. Novak, E.J.; Liu, A.W.; Nepom, G.T.; Kwok, W.W. MHC class II tetramers identify peptide-specific human $\mathrm{CD} 4(+) \mathrm{T}$ cells proliferating in response to influenza A antigen. J. Clin. Investig. 1999, 104, R63-R67.

50. Kwok, W.W.; Gebe, J.A.; Liu, A.; Agar, S.; Ptacek, N.; Hammer, J.; Koelle, D.M.; Nepom, G.T. Rapid epitope identification from complex class-II-restricted T-cell antigens. Trends Immunol. 2001, 22, 583-588.

51. Long, H.M.; Chagoury, O.L.; Leese, A.M.; Ryan, G.B.; James, E.; Morton, L.T.; Abbott, R.J.; Sabbah, S.; Kwok, W.; Rickinson, A.B. MHC II tetramers visualize human CD4+ T cell responses to Epstein-Barr virus infection and demonstrate atypical kinetics of the nuclear antigen EBNA1 response. J. Exp. Med. 2013, 210, 933-949.

52. Petersen, S.H.; Odintsova, E.; Haigh, T.A.; Rickinson, A.B.; Taylor, G.S.; Berditchevski, F. The role of tetraspanin CD63 in antigen presentation via MHC class II. Eur. J. Immunol. 2011, $41,2556-2561$.

53. Neudorfer, J.; Schmidt, B.; Huster, K.M.; Anderl, F.; Schiemann, M.; Holzapfel, G.; Schmidt, T.; Germeroth, L.; Wagner, H.; Peschel, C.; et al. Reversible HLA multimers (Streptamers) for the isolation of human cytotoxic $\mathrm{T}$ lymphocytes functionally active against tumor- and virus-derived antigens. J. Immunol. Methods 2007, 320, 119-131.

54. Manz, R.; Assenmacher, M.; Pfluger, E.; Miltenyi, S.; Radbruch, A. Analysis and sorting of live cells according to secreted molecules, relocated to a cell-surface affinity matrix. Proc. Natl. Acad. Sci. USA 1995, 92, 1921-1925.

55. Chattopadhyay, P.K.; Yu, J.; Roederer, M. Live-cell assay to detect antigen-specific CD4+ T-cell responses by CD154 expression. Nat. Protoc. 2006, 1, 1-6.

56. Frentsch, M.; Arbach, O.; Kirchhoff, D.; Moewes, B.; Worm, M.; Rothe, M.; Scheffold, A.; Thiel, A. Direct access to CD4+ T cells specific for defined antigens according to CD154 expression. Nat. Med. 2005, 11, 1118-1124.

57. Hsu, D.C.; Kerr, S.J.; Iampornsin, T.; Pett, S.L.; Avihingsanon, A.; Thongpaeng, P.; Zaunders, J.J.; Ubolyam, S.; Ananworanich, J.; Kelleher, A.D.; et al. Restoration of CMV-specific-CD4 T cells with ART occurs early and is greater in those with more advanced immunodeficiency. PLOS ONE 2013, 8, e77479.

58. Zaunders, J.J.; Munier, M.L.; Seddiki, N.; Pett, S.; Ip, S.; Bailey, M.; Xu, Y.; Brown, K.; Dyer, W.B.; Kim, M.; et al. High levels of human antigen-specific CD4+ T cells in peripheral blood revealed by stimulated coexpression of CD25 and CD134 (OX40). J. Immunol. 2009, 183, $2827-2836$.

59. Seddiki, N.; Cook, L.; Hsu, D.C.; Phetsouphanh, C.; Brown, K.; Xu, Y.; Kerr, S.J.; Cooper, D.A.; Munier, C.M.; Pett, S.; et al. Human antigen-specific CD4(+) CD25(+) CD134(+) CD39(+) $\mathrm{T}$ cells are enriched for regulatory $\mathrm{T}$ cells and comprise a substantial proportion of recall responses. Eur. J. Immunol. 2014, 44, 1644-1661. 
60. Hsu, D.C.; Kerr, S.J.; Thongpaeng, P.; Iampornsin, T.; Pett, S.L.; Zaunders, J.J.; Avihingsanon, A.; Ubolyam, S.; Ananworanich, J.; Kelleher, A.D.; et al. Incomplete restoration of Mycobacterium tuberculosis-specific-CD4 T cell responses despite antiretroviral therapy. J. Infect. 2014, 68, 344-354.

61. Keoshkerian, E.; Helbig, K.; Beard, M.; Zaunders, J.; Seddiki, N.; Kelleher, A.; Hampartzoumian, T.; Zekry, A.; Lloyd, A.R. A novel assay for detection of hepatitis $\mathrm{C}$ virus-specific effector CD4(+) $\mathrm{T}$ cells via co-expression of CD25 and CD134. J. Immunol. Methods 2012, 375, 148-158.

62. Phetsouphanh, C.; Xu, Y.; Bailey, M.; Pett, S.; Zaunders, J.; Seddiki, N.; Kelleher, A.D. Ratios of effector to central memory antigen-specific CD4(+) T cells vary with antigen exposure in HIV+ patients. Immunol. Cell Biol. 2014, 92, 384-388.

63. Zuba-Surma, E.K.; Kucia, M.; Abdel-Latif, A.; Lillard, J.W.J.; Ratajczak, M.Z. The ImageStream System: A key step to a new era in imaging. Folia. Histochem. Cytobiol. 2007, 45, 279-290.

64. Beum, P.V.; Lindorfer, M.A.; Hall, B.E.; George, T.C.; Frost, K.; Morrissey, P.J.; Taylor, R.P. Quantitative analysis of protein co-localization on B cells opsonized with rituximab and complement using the ImageStream multispectral imaging flow cytometer. J. Immunol. Methods 2006, 317, 90-99.

65. George, T.C.; Basiji, D.A.; Hall, B.E.; Lynch, D.H.; Ortyn, W.E.; Perry, D.J.; Seo, M.J.; Zimmerman, C.A.; Morrissey, P.J. Distinguishing modes of cell death using the ImageStream multispectral imaging flow cytometer. Cytometry A 2004, 59, 237-245.

66. Lindstrom, S.; Andersson-Svahn, H. Overview of single-cell analyses: Microdevices and applications. Lab Chip 2010, 10, 3363-3372.

67. Torres, A.J.; Contento, R.L.; Gordo, S.; Wucherpfennig, K.W.; Love, J.C. Functional single-cell analysis of T-cell activation by supported lipid bilayer-tethered ligands on arrays of nanowells. Lab Chip 2013, 13, 90-99.

68. Varadarajan, N.; Julg, B.; Yamanaka, Y.J.; Chen, H.; Ogunniyi, A.O.; McAndrew, E.; Porter, L.C.; Piechocka-Trocha, A.; Hill, B.J.; Douek, D.C.; et al. A high-throughput single-cell analysis of human $\mathrm{CD} 8(+) \mathrm{T}$ cell functions reveals discordance for cytokine secretion and cytolysis. J. Clin. Investig. 2011, 121, 4322-4331.

69. Varadarajan, N.; Kwon, D.S.; Law, K.M.; Ogunniyi, A.O.; Anahtar, M.N.; Richter, J.M.; Walker, B.D.; Love, J.C. Rapid, efficient functional characterization and recovery of HIV-specific human CD8+ T cells using microengraving. Proc. Natl. Acad. Sci. USA 2012, 109, 3885-3890.

70. Dura, B.; Voldman, J. Spatially and temporally controlled immune cell interactions using microscale tools. Curr. Opin. Immunol. 2015, 35, 23-29.

71. Elitas, M.; Brower, K.; Lu, Y.; Chen, J.J.; Fan, R. A microchip platform for interrogating tumor-macrophage paracrine signaling at the single-cell level. Lab Chip 2014, 14, 3582-3588.

72. Kravchenko-Balasha, N.; Wang, J.; Remacle, F.; Levine, R.D.; Heath, J.R. Glioblastoma cellular architectures are predicted through the characterization of two-cell interactions. Proc. Natl. Acad. Sci. USA 2014, 111, 6521-6526. 
73. Vanherberghen, B.; Olofsson, P.E.; Forslund, E.; Sternberg-Simon, M.; Khorshidi, M.A.; Pacouret, S.; Guldevall, K.; Enqvist, M.; Malmberg, K.J.; Mehr, R.; et al. Classification of human natural killer cells based on migration behavior and cytotoxic response. Blood 2013, 121, 1326-1334.

74. Dura, B.; Dougan, S.K.; Barisa, M.; Hoehl, M.M.; Lo, C.T.; Ploegh, H.L.; Voldman, J. Profiling lymphocyte interactions at the single-cell level by microfluidic cell pairing. Nat. Commun. 2015, 6,5940 .

75. Lindstrom, S.; Mori, K.; Ohashi, T.; Andersson-Svahn, H. A microwell array device with integrated microfluidic components for enhanced single-cell analysis. Electrophoresis 2009, 30, 4166-4171.

76. Lecault, V.; White, A.K.; Singhal, A.; Hansen, C.L. Microfluidic single cell analysis: From promise to practice. Curr. Opin. Chem. Biol. 2012, 16, 381-390.

77. White, A.K.; VanInsberghe, M.; Petriv, O.I.; Hamidi, M.; Sikorski, D.; Marra, M.A.; Piret, J.; Aparicio, S.; Hansen, C.L. High-throughput microfluidic single-cell RT-qPCR. Proc. Natl. Acad. Sci. USA 2011, 108, 13999-14004.

78. Marcus, J.S.; Anderson, W.F.; Quake, S.R. Microfluidic single-cell mRNA isolation and analysis. Anal. Chem. 2006, 78, 3084-3089.

79. Marcus, J.S.; Anderson, W.F.; Quake, S.R. Parallel picoliter rt-PCR assays using microfluidics. Anal. Chem. 2006, 78, 956-958.

80. Teles, J.; Enver, T.; Pina, C. Single-cell PCR profiling of gene expression in hematopoiesis. Methods Mol. Biol. 2014, 1185, 21-42.

81. Whale, A.S.; Huggett, J.F.; Cowen, S.; Speirs, V.; Shaw, J.; Ellison, S.; Foy, C.A.; Scott, D.J. Comparison of microfluidic digital PCR and conventional quantitative PCR for measuring copy number variation. Nucleic Acids Res. 2012, 40, e82.

82. Moltzahn, F.; Hunkapiller, N.; Mir, A.A.; Imbar, T.; Blelloch, R. High throughput microRNA profiling: Optimized multiplex qRT-PCR at nanoliter scale on the fluidigm dynamic arrayTM IFCs. J. Vis. Exp. 2011, 54, 2552.

83. Jang, J.S.; Simon, V.A.; Feddersen, R.M.; Rakhshan, F.; Schultz, D.A.; Zschunke, M.A.; Lingle, W.L.; Kolbert, C.P.; Jen, J. Quantitative miRNA expression analysis using fluidigm microfluidics dynamic arrays. BMC Genom. 2011, 12, 144.

84. Mingueneau, M.; Kreslavsky, T.; Gray, D.; Heng, T.; Cruse, R.; Ericson, J.; Bendall, S.; Spitzer, M.H.; Nolan, G.P.; Kobayashi, K.; et al. The transcriptional landscape of alphabeta T cell differentiation. Nat. Immunol. 2013, 14, 619-632.

85. Johnson, S.; Eller, M.; Teigler, J.E.; Maloveste, S.M.; Schultz, B.T.; Soghoian, D.Z.; Lu, R.; Oster, A.F.; Chenine, A.L.; Alter, G.; et al. Cooperativity of HIV-specific cytolytic CD4+ T cells and CD8+ T cells in control of HIV viremia. J. Virol. 2015, 89, 7494-7505.

86. Yin, H.; Marshall, D. Microfluidics for single cell analysis. Curr. Opin. Biotechnol. 2012, 23, 110-119.

87. Shalek, A.K.; Satija, R.; Shuga, J.; Trombetta, J.J.; Gennert, D.; Lu, D.; Chen, P.; Gertner, R.S.; Gaublomme, J.T.; Yosef, N.; et al. Single-cell RNA-seq reveals dynamic paracrine control of cellular variation. Nature 2014, 510, 363-369. 
88. Saliba, A.E.; Westermann, A.J.; Gorski, S.A.; Vogel, J. Single-cell RNA-seq: Advances and future challenges. Nucleic Acids Res. 2014, 42, 8845-8860.

89. Shalek, A.K.; Satija, R.; Adiconis, X.; Gertner, R.S.; Gaublomme, J.T.; Raychowdhury, R.; Schwartz, S.; Yosef, N.; Malboeuf, C.; Lu, D.; et al. Single-cell transcriptomics reveals bimodality in expression and splicing in immune cells. Nature 2013, 498, 236-240.

90. Quake, T.K.S.R. Single-cell genomics. Nat. Methods 2011, 8, 311-314.

91. Mahata, B.; Zhang, X.; Kolodziejczyk, A.A.; Proserpio, V.; Haim-Vilmovsky, L.; Taylor, A.E.; Hebenstreit, D.; Dingler, F.A.; Moignard, V.; Gottgens, B.; et al. Single-cell RNA sequencing reveals $\mathrm{T}$ helper cells synthesizing steroids de novo to contribute to immune homeostasis. Cell Rep. 2014, 7, 1130-1142.

92. Han, A.; Glanville, J.; Hansmann, L.; Davis, M.M. Linking T-cell receptor sequence to functional phenotype at the single-cell level. Nat. Biotechnol. 2014, 32, 684-692. 


\title{
Evidence for P-Glycoprotein Involvement in Cell Volume Regulation Using Coulter Sizing in Flow Cytometry
}

\author{
Jennifer Pasquier, Damien Rioult, Nadine Abu-Kaoud, Jessica Hoarau-Véchot, \\ Matthieu Marin and Frank Le Foll
}

\begin{abstract}
The regulation of cell volume is an essential function that is coupled to a variety of physiological processes such as receptor recycling, excitability and contraction, cell proliferation, migration, and programmed cell death. Under stress, cells undergo emergency swelling and respond to such a phenomenon with a regulatory volume decrease (RVD) where they release cellular ions, and other osmolytes as well as a concomitant loss of water. The link between P-glycoprotein, a transmembrane transporter, and cell volume regulation is controversial, and changes in cells volume are measured using microscopy or electrophysiology. For instance, by using the patch-clamp method, our team demonstrated that chloride currents activated in the RVD were more intense and rapid in a breast cancer cell line overexpressing the P-glycoprotein (P-gp). The Cell Lab Quanta SC is a flow cytometry system that simultaneously measures electronic volume, side scatter and three fluorescent colors; altogether this provides unsurpassed population resolution and accurate cell counting. Therefore, here we propose a novel method to follow cellular volume. By using the Coulter-type channel of the cytometer Cell Lab Quanta SC MPL (multiplatform loading), we demonstrated a role for the P-gp during different osmotic treatments, but also a differential activity of the P-gp through the cell cycle. Altogether, our data strongly suggests a role of P-gp in cell volume regulation.
\end{abstract}

Reprinted from Int. J. Mol. Sci. Cite as: Pasquier, J.; Rioult, D.; Abu-Kaoud, N.; Hoarau-Véchot, J.; Marin, M.; Le Foll, F. Evidence for P-Glycoprotein Involvement in Cell Volume Regulation Using Coulter Sizing in Flow Cytometry. Int. J. Mol. Sci. 2015, 16, 14318-14337.

\section{Introduction}

Each living cell type seems to have a form and volume that is well defined, and determined by the size of their cytoplasmic membrane as well as their cytosolic content. However, variations of osmotic pressures lead to cell volume regulation [1,2]. In the hypotonic or hypertonic context, the ability of cells to regulate their volume in a short period of time (around a minute) in order to avoid swelling or shrinkage is a fundamental mechanism [3]. Under hypo-osmotic conditions, cells are able to escape a burst after swelling by activation of a mechanism known as regulatory volume decrease (RVD) [4]. Interestingly, even under a regular osmotic pressure, the cell volume is fluctuating in response to cell events such as cell proliferation, migration, glycolysis or cell death [5-8].

P-glycoprotein (P-gp, $A B C B 1$ ), a member of the ATP-binding cassette (ABC) superfamily of transporters, is a $170-\mathrm{kD}$ transmembrane glycoprotein involved in the multi-drug resistance (MDR) phenotype $[9,10]$. The link between P-gp expression and cell volume regulation has been widely debated over the past decades $[1,11,12]$. Some studies reported that the P-gp is able to increase 
the magnitude of cell volume activated chloride currents, and so modulate the RVD [13-16]. On the contrary, other studies did not find any functional correlation between P-gp and volume regulation [17-20]. In 2005, our team demonstrated the role of P-gp in the regulation of volume activated chloride currents using wild-type human breast cancer MCF7 cells, and a doxorubicin-selected MDR variants [21]. Recently, it has been shown that apoptotic resistance in MDR P-gp overexpressing ascite tumour cells, involved impairment of the apoptotic volume decrease (AVD), an apoptotic event similar to the RVD [22]. Finally, in 2012, a team working on hepatocytes of the fish, Sparus aurata, suggested the involvement of the P-gp in the RVD [23].

The difference between all these studies could originate from the models and the methods used. Therefore, technological challenges still exist and the technique's accuracy to measure both cell volume and P-gp activity/expression seems essential.

In this paper, we develop an original method based on flow cytometry analysis to study the role of the P-gp in the RVD in response to hypo-osmotic shocks. By using the Coulter-type channel of the cytometer Cell Lab Quanta SC MPL, we were able to follow accurately the electronic volume (EV) of the cells during either osmotic and/or pharmacologic treatment types.

\section{Results}

\subsection{Resistant and Sensitive Variants Display Different Cell Volumes, Shapes and}

\section{Membrane Capacitance}

Our group previously studied P-gp overexpression and activity in the MCF7/Doxo variant [24-26]; cell volumes of MCF7 and MCF7/Doxo were measured using different methods (Figure 1). In classical microscopy, morphological differences between the two variants could be verified (Figure 1A). The MCF7 appeared more birefringent and round, whereas the MCF7/Doxo were more flat and spread. Using Hoffman modulation contrast imaging on a freshly plated 50:50 co-culture, we revealed a clear morphological difference between MCF7 and MCF7/Doxo (Figure 1B top panel). After four days of co-culture, it was possible to observe a stable and unique spatial organization with the formation of MCF7 islets surrounded by MCF7/Doxo (Figure 1B bottom panel).

These differences have been confirmed using the Cell Lab Quanta MPL flow cytometer. This system exploits the Coulter principle for an accurate volume determination instead of the low-angle laser light scattering technique implemented in most of the cytometers. In short, as particles suspended in a saline solution are drawn through the small aperture of an insulated electrical sensor, they displace an equal volume of electrolyte solution that creates a resistance and leads to a voltage pulse. The voltage pulse intensity is proportional to the particle volume, thus called Electronic Volume (EV). Figure 1C displays the diameter (in $\mu \mathrm{m}$ ) of the cells in suspension in a $300 \mathrm{mOsmol} / \mathrm{kg} \mathrm{H}_{2} \mathrm{O}$. A statistical analysis performed on more than 300 measurements revealed that the MCF7 were smaller in diameter and volume compared to the MCF7/Doxo (Figure 1D). It is also interesting to note that the MCF7 seemed to be less homogeneous in size than the MCF7/Doxo as revealed by the variation coefficient for the diameters $(40.03 \% \pm 0.05 \%$ and $30.85 \% \pm 0.85 \%$, respectively). 
Finally, using electrophysiology, we measured the whole-cell capacitance of the two variants, $20.7 \pm 1.4 \mathrm{pF}(n=19)$ for MCF7 and $29.4 \pm 2.1 \mathrm{pF}(n=33)$ for MCF7/Doxo, respectively (Figure 1E). Considering a constant specific capacitance of $\mathrm{Cs}_{\mathrm{S}}=1 \mu \mathrm{F} / \mathrm{cm}^{2}$ [27] for the plasma membrane, these results indicate that the membrane electric surface is higher in the MCF7/Doxo compared to wild-type. This observation seems to contradict the one obtained by the volume Coulter (Figure 1F).

A
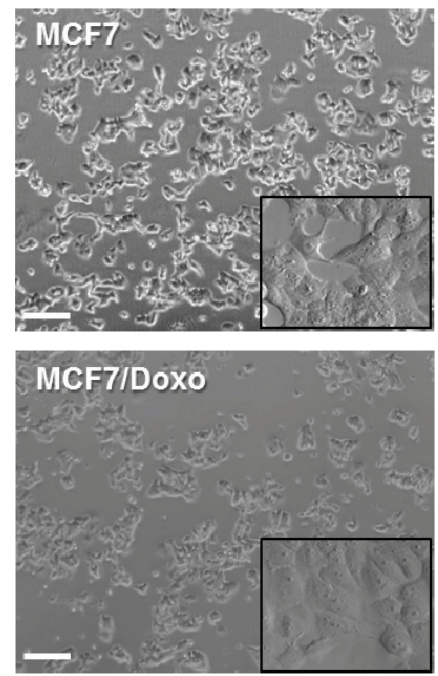

D

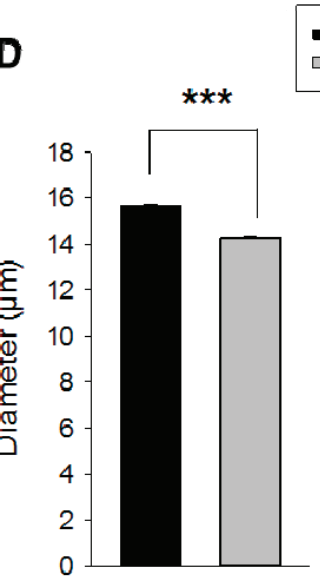

B
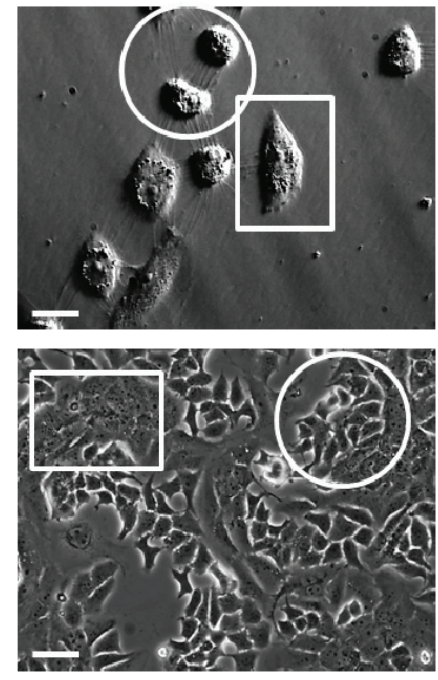

E
C
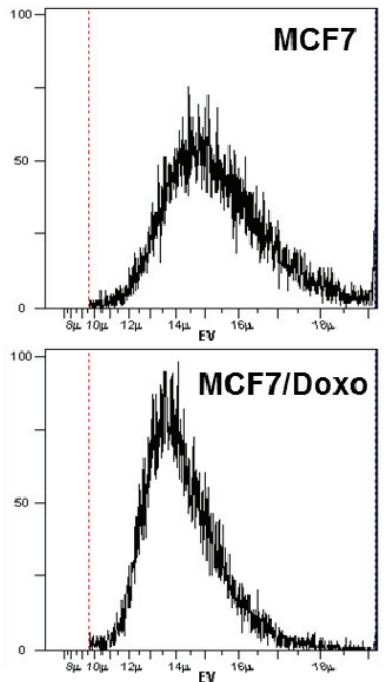

MCF7 $(n=185)$

MCF7/Doxo $(n=185)$

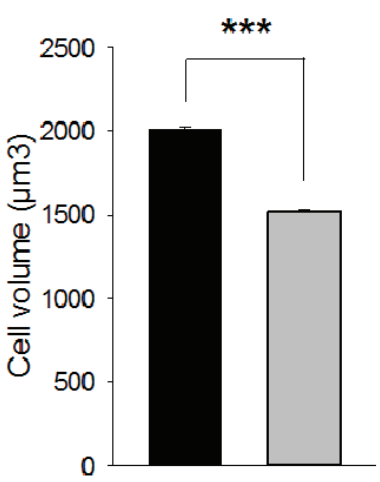

MCF7 $(n=19)$ MCF7/DOXO $(n=33)$

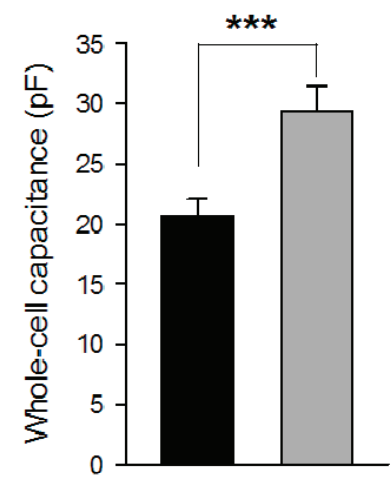

Figure 1. Cont. 
F

\begin{tabular}{|c|c|c|c|c|}
\hline & \multicolumn{2}{|c|}{ Patch Clamp } & \multicolumn{2}{c|}{ Flow cytometry } \\
\hline & MCF7 & MCF7/Doxo & MCF7 & MCF7/Doxo \\
\hline Capacitance $(\mathrm{pF})$ & $\mathbf{2 0 . 7}$ & $\mathbf{2 9 . 4}$ & ----- & ---- \\
\hline Surface $\left(\mathrm{cm}^{2}\right)$ & $2.07 \times 10^{-5}$ & $2.94 \times 10^{-5}$ & $1.89 \times 10^{-6}$ & $1.54 \times 10^{-6}$ \\
\hline Radius $(\mu \mathrm{m})$ & 25.67 & 30.59 & 7.80 & 7.11 \\
\hline Volume $\left(\mu \mathrm{m}^{3}\right)$ & 70847 & 119918 & 2004 & 1513 \\
\hline
\end{tabular}

Figure 1. MCF7 and MCF7/Doxo display different morphological features. (A) Spatial organization of MCF7 and MCF7/Doxo in phase contrast microscopy. Scale bar: $50 \mu \mathrm{m}$; (B) Hoffman modulation contrast (top) and phase contrast micrographs (bottom) of MCF-7 and MCF-7/Doxo in co-cultures. Dishes were seeded with a 50:50 mixture of MCF-7:MCF7/Doxo at day zero. Morphological differences permit an immediate identification of each cell subpopulation islets. MCF7 appeared birefringent and round (rounds) whereas MCF-7/Doxo are more flat and spread (square). Scale bar: $20 \mu \mathrm{m}$; (C) Cell volume of MCF7 and MCF7/Doxo in flow cytometry. The electronic volume (EV) was determined by the flow cytometer according to the Coulter Principle; (D) Histograms giving the mean cell diameter (left) and volume (right) for 185 repeated experiments. The MCF7 appear significantly bigger than the MCF7/Doxo. Data are presented as mean \pm SEM; (E) Whole-cell capacitance measurements of MCF7 and MCF7/Doxo. MCF7/Doxo display a higher whole-cell capacitance than the MCF7. Data are presented as mean \pm SEM; (F) Summary of the different size measured or calculated for the MCF7 and MCF7/Doxo. *** $p<0.001$.

\subsection{Cell Volume Monitoring during Hypo-Osmotic Shocks}

Flow cytometry coupled to Coulter EV measurements represents a valuable approach to monitor cell size variations in real-time. Thus, we have used this possibility to carry out analysis of volume change time-course of cells undergoing osmotic challenges in suspension at a low flow rate ( $25 \mu \mathrm{L} / \mathrm{min}$ ). With these settings, the cell volume distributions can be determined over $20 \mathrm{~min}$. This approach is better than the traditional volume coulter method that allows only a static measurement of the cell volumes. As shown in Figure 2A, cell volumes of the two variants were stable over the $20 \mathrm{~min}$ period. However, during $50 \%$ hypo-osmotic shocks $(150 \mathrm{mOsmol} / \mathrm{kg} \mathrm{H} \mathrm{O})$, a significant swelling of both variants was detected two minutes after the substitution of the isotonic solution with the hypotonic one (Figure 2B). The temporal monitoring of the volume compensation, RVD, revealed important differences between the two variants (Figure 2C). While the MCF7/Doxo cells were able to compensate the swelling drove by hypotonicity, the MCF7 cells could not. For the MCF7/Doxo cells, cell volume normalization appeared after less than 10 min, whilst no RVD mechanism was noticed after 20 min for the MCF7 cells.

This experiment has been repeated several times in isotonic and hypotonic conditions. To analyze the large number of points generated (200,000 cells analyzed/experiment), 20 successive 
gates of 1-min cell volume continuous recording have been set (Figure 2D). For each 1-min interval, the mean cell volume has been determined. In these conditions, it has been possible to draw a graph representative of the different experiments (Figure 2E,F). After $20 \mathrm{~min}$, the MCF7 were not able to display any RVD mechanism, neither under 25 nor under 50\% hypo-osmotic shocks (Figure 2E). On the contrary, in the MCF7/Doxo cells, a RVD was set up immediately and re-established normotonic volume values after $7 \mathrm{~min}$ in both $25 \%$ and $50 \%$ hypo-osmotic shocks (Figure 2F).

A

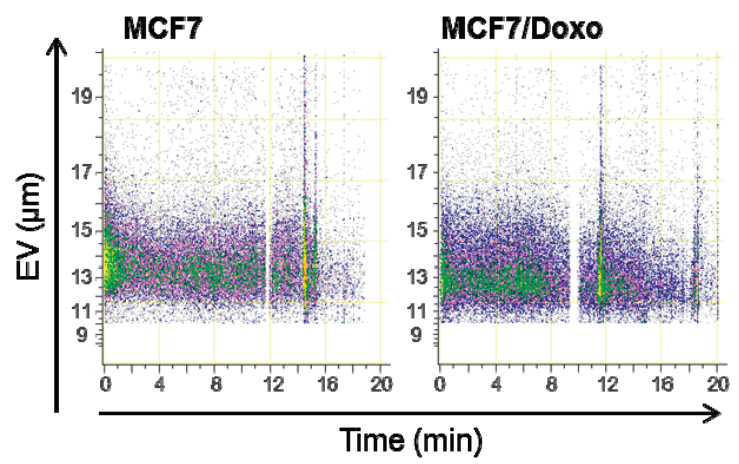

C

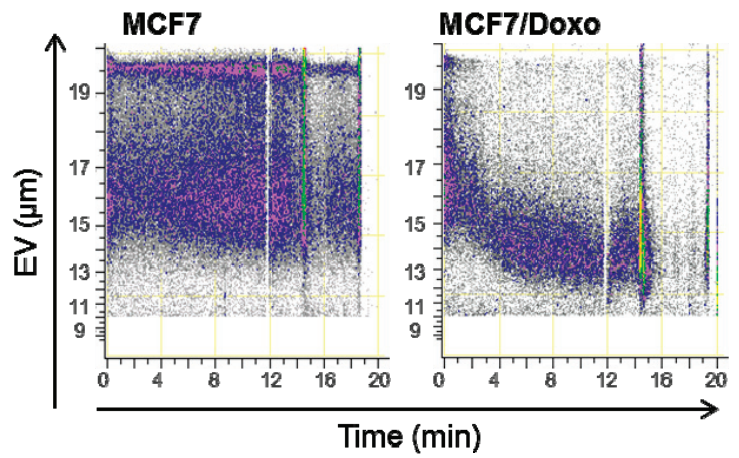

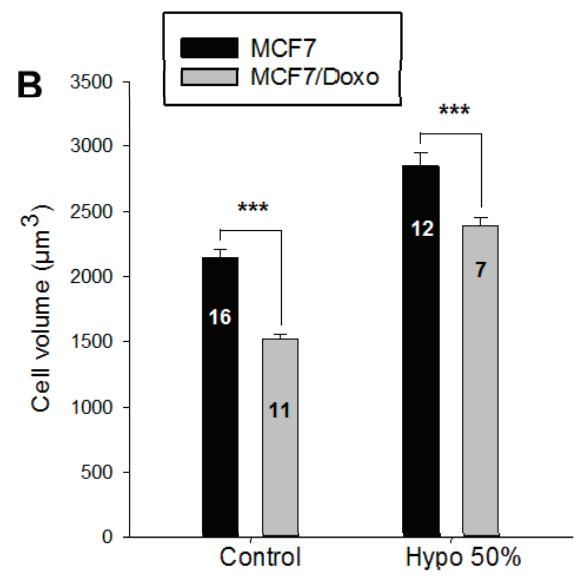

D

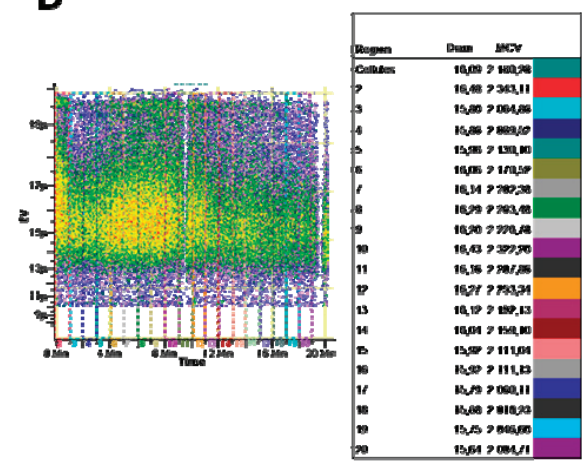

Figure 2. Cont. 

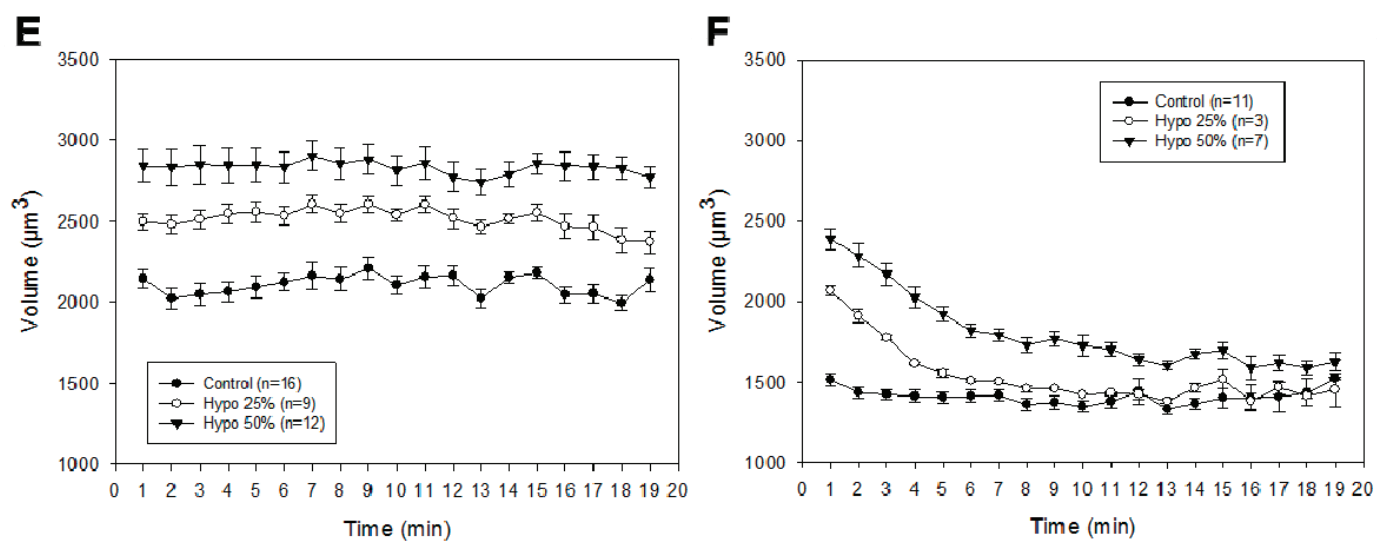

Figure 2. Cell volume monitoring during hypo-osmotic shocks. (A) Cell volume monitoring of MCF7 and MCF7/Doxo cells in normotonic conditions. The two graphs represent flow cytometry plots of the electronic volume (EV) vs. time, during the 20 min of the experiment; (B) Cell volumes after one minute of hypo-osmotic stress. Cell volumes were recorded by flow cytrometry before (control) or after one minute of a 50\% hypo-osmotic shock (Hypo 50\%). Data are presented as mean $\pm \mathrm{SEM}$; (C) Cell volumes monitoring in MCF7 and MCF7/Doxo cells during a hypo-osmotic shocks. The two flow cytometry plots represent the electronic volume (EV) of the cells subjected to a 50\% hypo-osmotic stress during the $20 \mathrm{~min}$. MCF7 cells (left plot) increased their volume without any compensation phenomenon. After a significant volume increase, MCF7/Doxo cells (right plot) retrieved their original volume; (D) Data extraction method. Nineteen equal gates of 1 min length have been created and applied to all samples. The mean cell diameter (Diam.) and of the mean cell volume (MCV) in each region have been extracted with the Cell Lab Quanta analysis software; (E) Cell volume monitoring of MCF7 under different conditions. Cell volumes of MCF7 have been recorded by flow cytometry in isotonic conditions (control) or during $25 \%$ or a $50 \%$ hypo-osmotic challenges; (F) Cell volume monitoring of MCF7/Doxo cells under different conditions. Cell volumes of MCF7 have been recorded by flow cytometry in isotonic conditions (control) or during $25 \%$ or a $50 \%$ hypo-osmotic challenges. $* * * p<0.001$.

\subsection{RVD in MCF7/Doxo Cells Is Dependent on P-gp Activity}

The role of efflux activity of P-gp in the regulation of the RVD has been investigated using different ligands of P-gp chosen for their different mechanisms of action. Thus, a non-competitive inhibitor, zosuquidar $(20 \mu \mathrm{M})$, a conformational monoclonal antibody, UIC2, and a P-gp substrate used in chemotherapy treatment of breast cancer, doxorubicin $(20 \mu \mathrm{M})$, have been used. First we quantified the effect of each compound on the P-gp efflux activity with the calcein-AM as a fluorescent allocrite probe (Figure 3A). Cells expressing high levels of P-gp rapidly extrude nonfluorescent calcein-AM from the plasma membrane. As a result, fluorescence intensity is 
inversely related to P-gp [26]. The conformational antibody UIC2 and zosuquidar completely abolished the P-gp activity in MCF7/Doxo cells, while doxorubicin did not alter the ability of MCF7/Doxo to expel calcein. In isotonic conditions, the cell volumes of MCF7/Doxo were not modified by any of the three ligands (data not shown). On the contrary, after $50 \%$ hypo-osmotic shocks, RVD was inhibited by the $3 \mathrm{P}$-gp modulators (Figure 3B). Analysis at 1 min shows clearly that the untreated MCF7/Doxo cells were already engaged in a RVD process while, in the presence of zosuquidar or doxorubicin, they did not even reach their maximal volume (Figure 3C). After $7 \mathrm{~min}$, the three ligands were able to significantly decrease RVD kinetics in MCF7/Doxo. Comparisons of the RVD rates revealed that doxorubicin slowed down most of the RVD process (Figure 3D). The experimental points of five different experiments, under doxorubicin treatment, were fitted with straight lines (Figure 3E). The small slope demonstrated the nearly total inhibition of the RVD in the presence of doxorubicin.

A

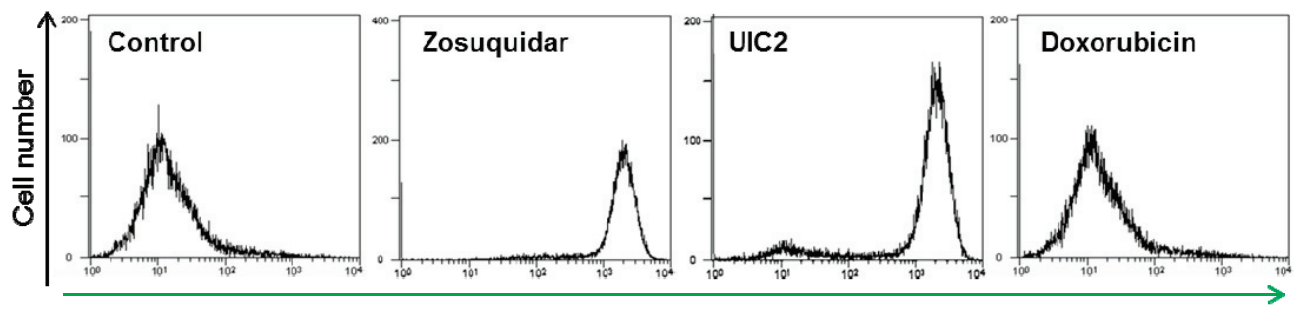

Calcein-AM

B
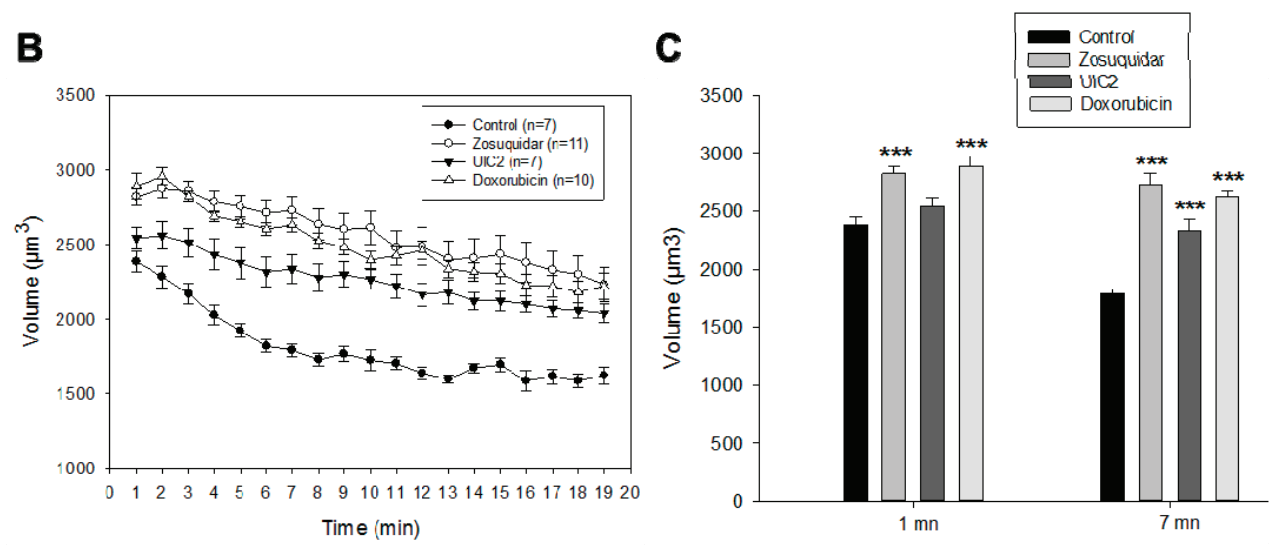

Figure 3. Cont. 

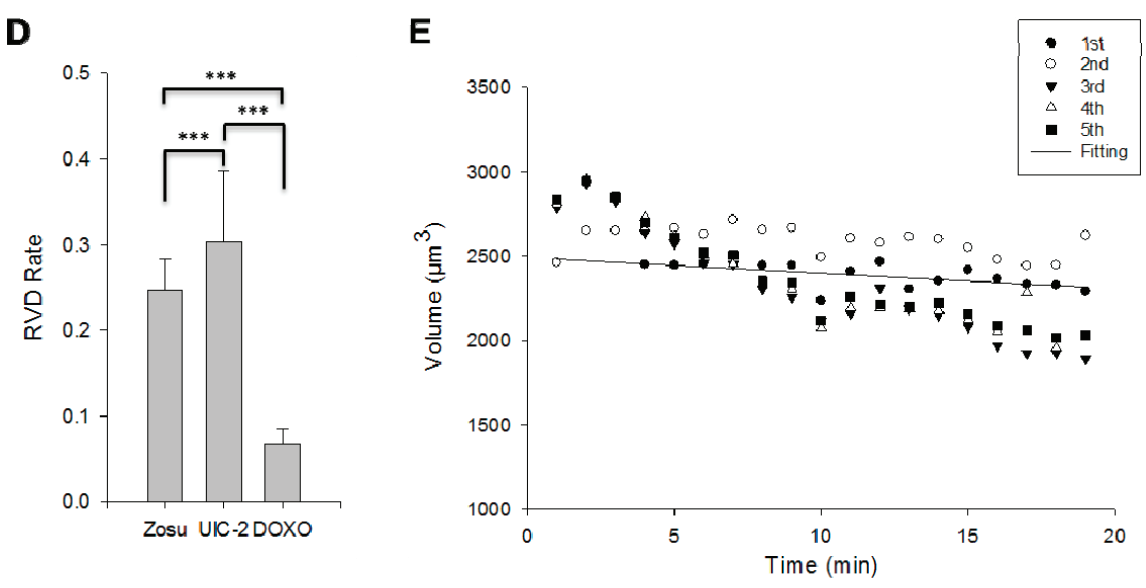

Figure 3. RVD in MCF7/Doxo is dependent on the P-gp activity. (A) P-gp activity. P-gp activity was followed with calcein-AM as a fluorescent probe. In each flow cytometry measurement, a sample of 10,000 cells was analyzed. Superimposed all-events histograms of calcein fluorescence distribution (log scale) in control MCF-7/Doxo cells (left plot) and MCF-7/Doxo pre-incubated with the P-gp antagonist zosuquidar and UIC2 (10 $\mu \mathrm{M}$, middle plots) or with doxorubicin (right histogram); (B) Cell volume monitoring of MCF7/Doxo in presence of P-gp modulators; (C) The histogram represents the mean of the cell volume after 1 or $7 \mathrm{~min}$ of $50 \%$ hypo-osmotic stress for the experiments presented in B. While under the control conditions (black) the cells retrieved their original volume after $7 \mathrm{~min}$, the cells in presence of the P-gp modulators were not able to compensate their volume increase. Data are presented as mean \pm SEM, $* * * p<0.001$; (D) RVD rate of MCF7/Doxo cells in 50\% hypo-osmotic shock (*** $p<0.001$ ); and (E) Graphs show MCF7/Doxo cell volumes from five independent samples analyzed by flow cytometry after $50 \%$ hypo-osmotic conditions in the presence of doxorubicin. The data were fitted with Sigma Plot.

\subsection{P-gp Activity and Cell Cycle}

In isotonic conditions, cell proliferation is one of the major events leading to cell size regulation. Thus, in the present work, cell volume changes were analyzed for the two variants during the cell cycle (Figure 4A). Measurements of large cell populations indicate that MCF7 exhibits larger size than MCF7/Doxo cells. In phase S or G2/M phases however, the volume of MCF7/Doxo was found to be higher than that of MCF7. In order to study the P-gp activity during the cell cycle, we developed a gating strategy based on the cell volumes defined by the cell cycle analysis (Figure 4B). However, cell size variations affect fluorescence signals and induce alteration in cell calcein content assessments. To overcome these potential variations, we have used the FL1-FC parameter of the cytometer, in which fluorescence intensity (FL1) was normalized as a ratio of fluorescence concentration to an accurate cell sizing determined by Coulter-type electronic volume (EV) [26]. Results indicate that the basal fluorescence of the calcein was more important in gate 1 
(corresponding to the cells in G0/G1), which correlates with a lower P-gp activity (Figure 4C). The best P-gp activity was detected in cells in G2/M which are the cells displaying the larger size. After this, we used a non-competitive P-gp inhibitor, the PSC833, and tested its efficacy on the calcein accumulation at the different stages of the cell cycle (Figure 4D). Dose response curves represented in Figure 4E allowed us to determine $\mathrm{EC}_{50}$ (half maximal effective concentration) and $E_{\max }$ (maximal effect) values of PSC833 for each gate. PSC833 maximal effect was increasing with the progression of the cells in the cell cycle, without any modifications of potency (EC50).

A
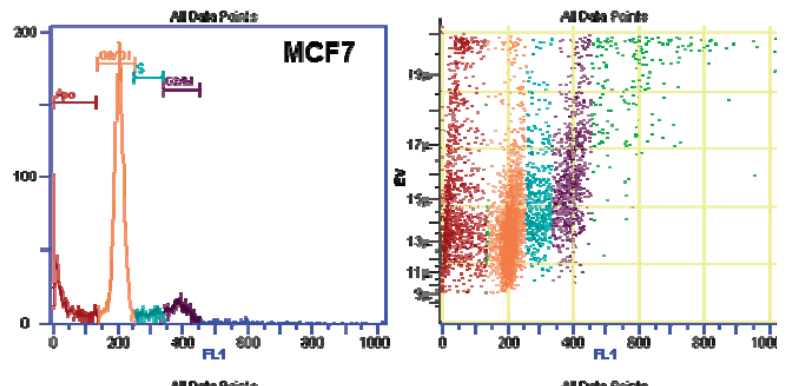

\begin{tabular}{|c|c|c|c|}
\hline Gate & Diameter & MCV & $\%$ total \\
\hline G0/G1 & 14.67 & 1654.47 & 64.98 \\
\hline S & 15.10 & 1802.82 & 5.34 \\
\hline G2/M & 15.87 & 2093.31 & 10.71 \\
\hline Apo & 13.24 & 1214.97 & 16.56 \\
\hline
\end{tabular}
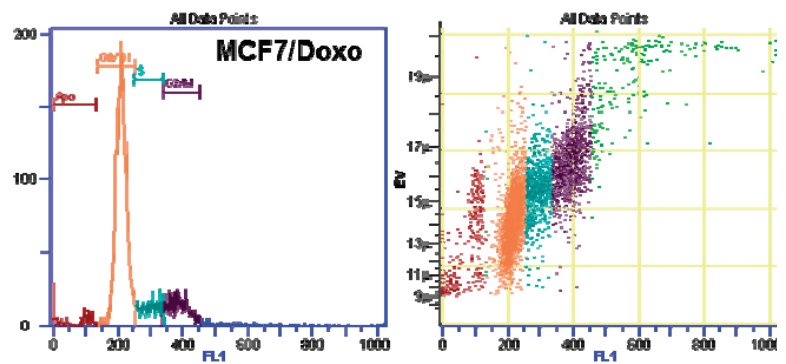

\begin{tabular}{|c|c|c|c|}
\hline Gate & Diameter & MCV & $\%$ total \\
\hline G0/G1 & 14.01 & 1439.31 & 69.05 \\
\hline S & 15.55 & 1969.75 & 10.71 \\
\hline G2/M & 16.49 & 2347.72 & 14.77 \\
\hline Apo & 13.27 & 1223.35 & 3.81 \\
\hline
\end{tabular}
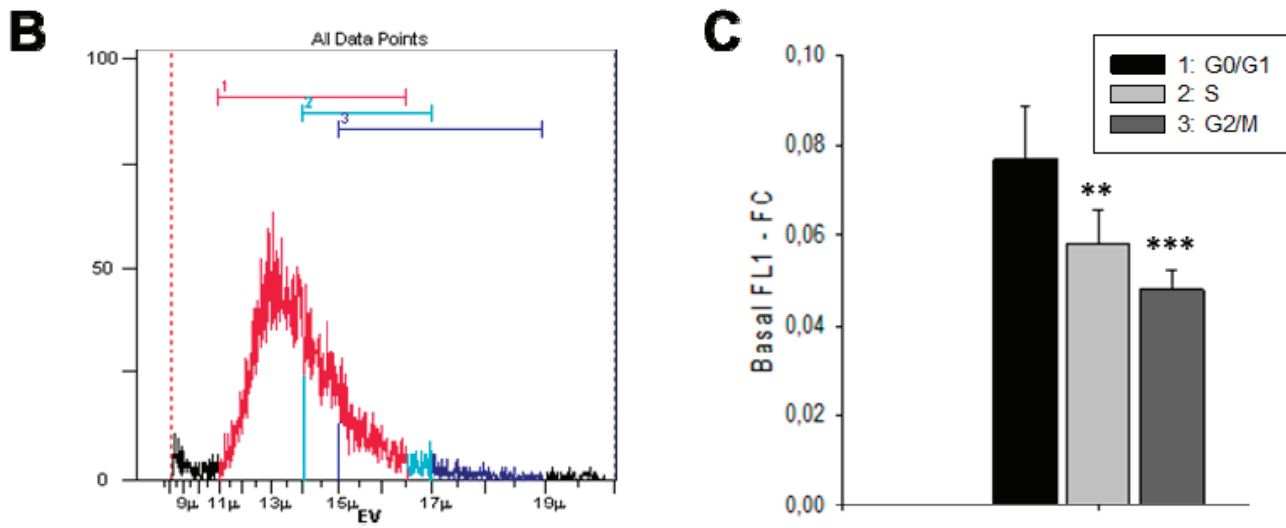

Figure 4. Cont. 
D

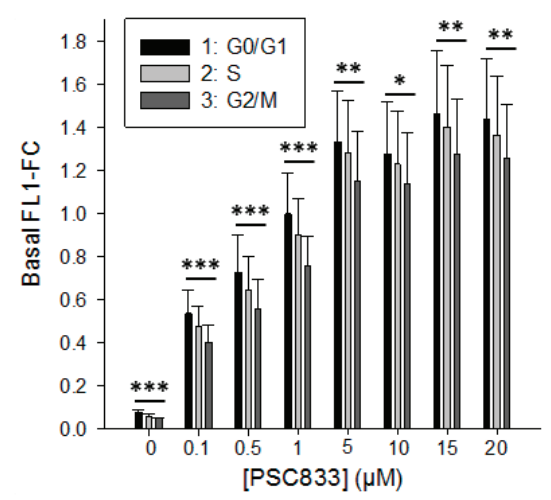

E

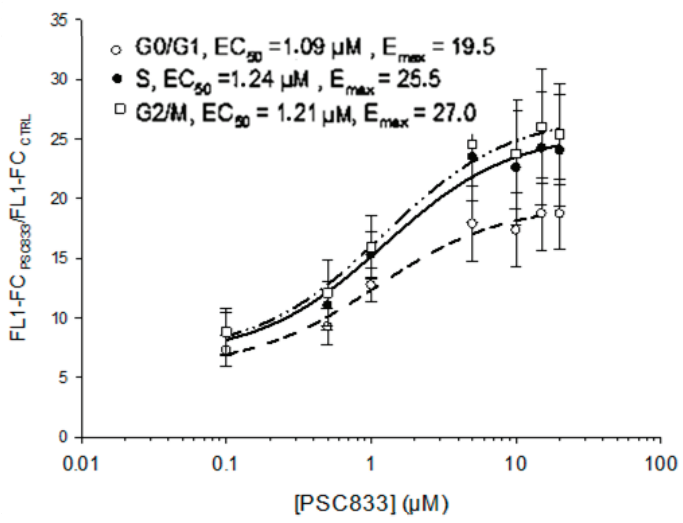

Figure 4. $\mathrm{MCF}-7 /$ Doxo cells exhibit a cell cycle-dependent P-gp activity. (A) Cell cycle of MCF7 and MCF7/Doxo was determined by DNA content measurements with the nucleic acid dye Hoechst 33342. Four gates corresponding to hypoploid particles (Apo), cells in $\mathrm{G} 0 / \mathrm{G} 1$, cells $\mathrm{S}$ or cells $\mathrm{G} 2 / \mathrm{M}$ phases were defined based on the FL1 level (left plots) and applied on the cell volume EV (right plots). The mean cell volume of the MCF7 and MCF7/Doxo were extracted from each gate (tables); (B) The gates defined in A on the FL1 parameter were switched on the EV channel to be applied to the following experiments; (C) P-gp activity within the cell cycle. The P-gp activity was assayed with calcein-AM and the amount of fluorescence per cell was expressed as FL1-FC (fluorescent light in channel 1-fluorescence concentration) which is the fluorescent light (FL) divided by the electronic volume (EV) determined by the flow cytometer according to the Coulter Principle; (D) P-gp inhibition with PSC833 within the cell cycle. Different concentrations of PSC833 were used and the P-gp activity was assayed with calcein-AM. The histogram represents the FL1-FC mean in each gate. The stars represent the difference between the three groups after an ANOVA test; and (E) PSC833 dose response curves expressed as mean FL1-FC. Each point represents mean \pm SEM (10 independent experiments) of FL1-FC, expressed as the ratio of signals in the presence of a blocker to signal in control conditions in each gate. Four-parameter logistic dose-response curves were fitted to the data to obtain blocker potencies (half-maximal effective concentration, $\mathrm{EC}_{50}$ ) and efficacies (maximum response, $E_{\max }$ ). Data are presented as mean \pm SEM with $n=10$ independent assays per data point. $* p<0.05, * * p<0.01, * * * p<0.001$.

\section{Discussion}

The role of P-gp in cell volume regulation has been discussed widely over the past 20 years. In the present study, we used an original, non-intrusive and label-free method based on flow cytometry coupled to Coulter volume determination to compare RVD in wild-type and doxorubicin-resistant MCF7, during hypo-osmotic challenges. This technique allowed us to follow 
not only the volume variation of cells in a live scenario, but also to concurrently study cell volumes and P-gp activity.

First, we demonstrated that MCF7 cells do not respond as MCF7/Doxo cells to hypo-osmotic shocks. In fact, while MCF7 cells underwent a persisting volume increase throughout the 20 min of the experiment, MCF7/Doxo cells were able to offset osmosis and to cancel swelling in less than ten minutes, suggesting strongly that the P-gp is promoting RVD. Historically, because of sequence similarities with an $\mathrm{ABC}$ transporter functioning as a chloride channel (the cystic fibrosis transmembrane regulator, CFTR), a role for P-gp in volume regulation has been investigated and, as a matter of fact, confirmed in P-gp overexpressing cells [13,28]. Thus, in 1992, Gill et al. stated that P-gp could form a channel itself, or a component of such a channel [28]. Two years later, Sadini and collaborators demonstrated in $m d r 1 / A B C B 1$ transfected NIH-3T3 cells that short-term hypotonic conditions caused an inhibition of P-gp activity [29]. In 1998, using a drug-sensitive cell line (MCF-7) and a P-gp-expressing derivative (BC19/3), the authors demonstrated an increase in the magnitude of cell volume activated chloride currents in $\mathrm{BC} 19 / 3$ cells, but ruled out the possibility of P-gp being itself the channel responsible for the volume-activated currents [14]. Conversely, other studies concluded that RVD and osmoregulatory chloride currents were not related to $\mathrm{P}$-gp expression in resistant cell lines or in cells transfected with $\mathrm{mdr} 1 / \mathrm{ABCB} 1$ transcripts $[17,19,20,30]$ or injected with the protein [31]. To add to the confusion, in 2002, Chen et al. abolished volume-activated chloride currents in bovine pigmented ciliary epithelial cells by using $m d r 1 / A B C B 1$ antisense oligonucleotides; suggesting their dependence on endogenous P-gp expression [32]. In 2005, using the same cell lines as in the present study, our team demonstrated that during hypotonic challenges, swelling-activated chloride currents were significantly activated faster and with larger densities in MCF7/Doxo cells than in MCF7 cells [21]. We also demonstrated the inhibition of this current by P-gp ligands, including conventional substrates such as doxorubicin, as well as antibodies. These results are in accordance with the total inhibition of RVD obtained in the presence of doxorubicin herein. In addition, our study is the first that combines non-invasive measurements of the cell volume and a cell model overexpressing the P-gp only by selection and not by transfection or injection.

More recently, in 2014, a relation between P-gp, AQP5 and drug resistance has been established [33]. By using AQP5-siRNA to silence AQP5 in the colon-derived cell line HT-29, the authors obtained a decrease of P-gp expression as well as of other actors of drug resistance, such as GST- $\pi$, and TOPO II. These findings are of prime importance since aquaporins are known to be involved in the early phase of apoptosis, characterized by a cell shrinkage, named apoptotic volume decrease (AVD) [34]. For instance, the under-expression of aquaporin AQP8 and 9, in hepatocellular carcinoma, is responsible for the resistance to starvation or TGF $\beta$-induced apoptosis [35]. Resistance to apoptosis is one of the principal features of tumor cells [22,36,37], hence we could hypothesize a role of P-gp in AVD-impairment as a mechanism associated with death evasion. In the presence of cytotoxins such as doxorubicin, and while pumping the substrate out of the cells, the P-gp could be involved in pathways counteracting cell shrinkage and may thus contribute to avoid apoptosis. Alteration of AVD could therefore be added to the classical mechanisms of resistance that the cancer cells use against chemotherapeutic agents. 
In parallel to the mechanisms of cell volume regulation in hypotonic conditions, we demonstrated obvious differences in term of morphology and cell volume between the two variants, in regular osmotic pressures. MCF7 cells in suspension in an isotonic solution have an average volume of about $2000 \mu \mathrm{m}^{3}$, while MCF7/Doxo cells have a much smaller volume of $1500 \mu \mathrm{m}^{3}$. This is in accordance with the results of Yang and collaborators demonstrating that human ovarian cancer cell line SKOV3 that started to express P-gp after a selection with cisplatin, exhibited dramatic changes in morphology, including reduction in cell size, loss of cellular projections and clustering [38]. In ascites tumor cell lines, a high correlation between the ability to pump out daunorubicin (by the P-gp), and the decrease in cell volume detected has also been demonstrated in resistant cell lines [39].

The measurement of the membrane capacitance in the whole cell configuration of the patch clamp technique revealed that the enclosed volume of the membrane is theoretically $70,847 \mu^{3}$ for MCF7 cells and 119,918 $\mu \mathrm{m}^{3}$ for MCF7/Doxo cells. This technique gives some information on the total area of the plasma membrane. Therefore, although the difference between the values obtained by Coulter volume and volume per surface area may seem significant, the folding structure of the membrane, which indeed contributes to the membrane capacity and not to the volume, could be an explanation. Thus, the measurements of the membrane capacitance suggest that the two variants have a different membrane conformation, whereby MCF-7/Doxo cells contain more membrane folding than MCF-7 cells (Figure 5). The volume differences between the two variants could also be explained by the difference in terms of morphology in phase contrast on adherent cells, since it was mentioned that MCF7 cells are more birefringent, so more swollen than MCF7/Doxo cells. We can hypothesize that through peculiar interactions with lipids and favorable locations in the plasma membrane, P-gp can influence the membrane structure [40-42].

Recently, many authors raised the possibility of a G1/S volume checkpoint controlling the progression through the cell cycle $[43,44]$. Even if the concept of a cell size checkpoint in the cell cycle has been well established in yeast and other organisms [45], it yet remains controversial in mammalian cells [46-48]. Some authors suggest that translational checkpoints at the G1/S transition could set a lower cell size limit [49-51], while others claim the importance of an upper limit of cell volume in progression through $\mathrm{M}$ phase [52,53]. Even if the cell volume regulation seems mandatory for the cell cycle progression and cell division, it remains unclear if these events are triggering the cell cycle itself or if they are just actors activated through the regular cyclin dependent kinase/cyclin (Cdk/cyclin) complex [43,44]. As per our results reported here, it seems that MCF7 cells are able to undergo a complete normal mitosis. Moreover, in a previous publication, we confirmed that there is no difference between the proliferation capacity of MCF7 and MCF7/Doxo, except the resistance to chemotherapy drugs exhibited by MCF7/Doxo cells [25]. Therefore, even if during the 20 min of hypotonic stress MCF7 cells were not able to display any efficient volume compensation, it seems that they are completely able to activate volume regulatory mechanisms in the cell cycle context. 


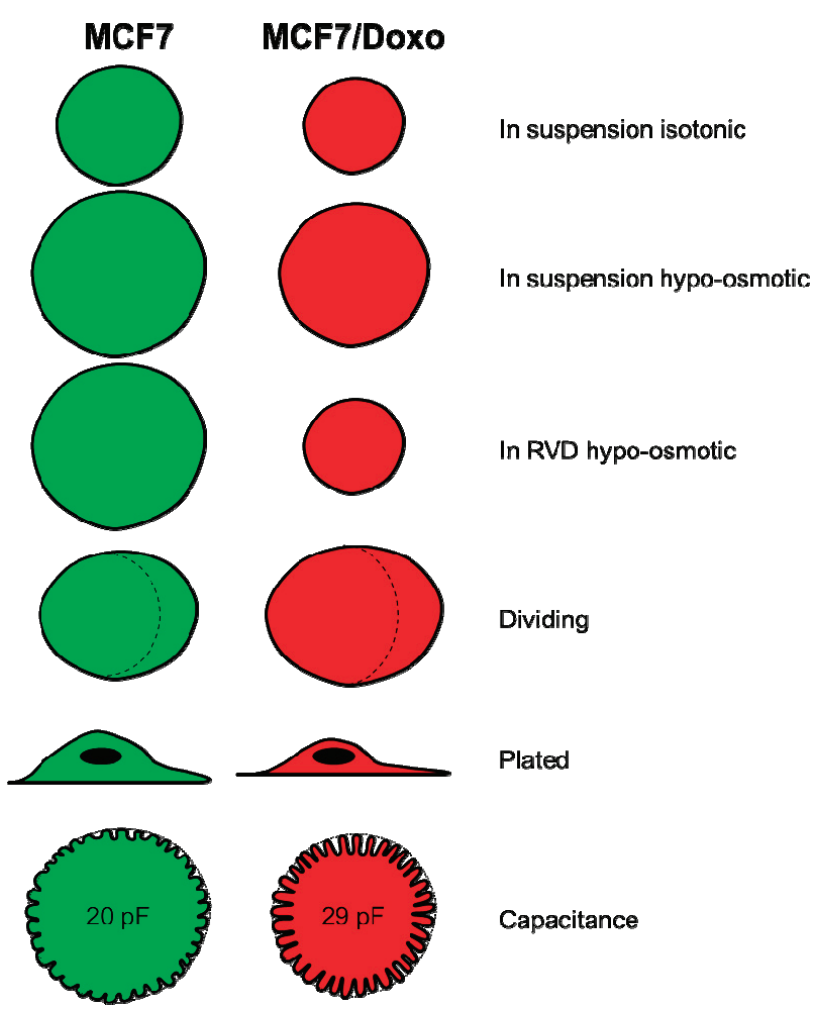

Figure 5. Schematic representation of MCF7 and MCF7/Doxo cells in each situation.

Finally, we were also able to show that the cells involved in mitosis display the highest P-gp activity. During mitosis, cells are subjected to constant volume variation that may reinforce the role of P-gp in cell volume regulation. Moreover, many drugs used in chemotherapy target cell division, and a high expression level of P-gp has been linked to resistance to these drugs $[54,55]$. We can thus suggest a new mechanism of resistance to this drug, independent of the drug efflux abilities of P-gp; this data therefore allows us to imagine the existence of new mechanistic consequences of P-gp overexpression.

\section{Material and Methods}

\subsection{Cell Cultures}

The study was carried out with human breast carcinoma derived cells, MCF-7 cells and its multi-drug resistant variant (MCF-7/Doxo); kindly provided by J.-P. Marie (Hôtel Dieu, Paris, France). MCF7/Doxo cells were isolated by stepwise selection with increasing concentrations of doxorubicin [56]. Cells were maintained in RPMI 1640 (Sigma, St. Louis, MO, USA) containing $5 \%$ of heat-inactivated fetal bovine serum (Sigma), $2 \mathrm{mM} \mathrm{L-glutamine} \mathrm{(Sigma,} \mathrm{St.} \mathrm{Louis,} \mathrm{MO,}$ USA), $1 \%$ antibiotic/antimycotic solution (Sigma), and incubated in a humidified atmosphere 
containing $5 \%$ of $\mathrm{CO}_{2}$ at $37{ }^{\circ} \mathrm{C}$. Doxorubicin $(1 \mu \mathrm{M})$ was added to the culture medium for the maintenance of the multi-drug resistant phenotype of MCF7/Doxo cells.

\subsection{Reagents}

All reagents were of the highest grade of purity and quality available. Purified doxorubicin (DOXO), DMSO, and phosphate buffer saline (PBS buffer, $\mathrm{pH}$ 7.4) were purchased from Sigma. Calcein acetoxy-methylester (calcéine-AM) was supplied by Invitrogen Life Technologies (Carlsbad, CA, USA). SDZ PSC833 and zosuquidar were kindly provided by J.-P. Marie (Hôtel Dieu, Paris, France). The final concentration of DMSO and $\mathrm{H}_{2} \mathrm{O}$ was less than $0.1 \%$.

\subsection{Flow Cytometry}

For P-gp expression, activity or cell cycle, the fluorescent light (FL) was quantified using a Cell Lab Quanta SC MPL flow cytometer (Beckman Coulter, Fullerton, CA, USA) equipped with a $22 \mathrm{~mW} 488 \mathrm{~nm}$ excitation laser. Voltage settings of photomultipliers were not modified throughout the experiments [24,25]. Each analysis consisted of a 10,000 events record, triggered on electronic volume (EV) as primary parameter, according to a particle diameter exceeding $8 \mu \mathrm{m}$.

For the volume monitoring, the cytometer was operated at a flow rate of $25 \mu \mathrm{L} / \mathrm{min}$. Cell sizes were accurately determined using the Coulter-type electronic volume (EV) channel of the cytometer, after calibration with 10- $\mu \mathrm{m}$ FlowCheck microspheres [57]. The cell volume distribution was measured for $20 \mathrm{~min}$.

\subsection{Osmostic Challenges and RVD Rate Calculation}

Osmolality of solutions was checked and adjusted by using a Wescor Vapro 5520 vapor pressure osmometer (Wescor Elitech, Logan, UT, USA). RVD rate is the time constant. It has been calculated using the Software Sigma Plot 11 (Systat Software Inc., Chicago, IL, USA). Data have been fitted with the Regression wizard using the Exponential Decay function. There are three parameters and the equation is $\mathrm{y}=\mathrm{y} 0+\mathrm{a} \times \mathrm{e}(-\mathrm{bx})$, where " $\mathrm{b}$ " is the decrease constant corresponding to the time constant represented in the bar graph (Figure 3D).

\subsection{P-gp Activity}

The calcein-AM efflux assay was used as previously described [26]. Briefly, resuspended cells were loaded with $0.25 \mu \mathrm{M}$ calcein acetoxy-methylester (Invitrogen) in RPMI for $15 \mathrm{~min}$ at $37^{\circ} \mathrm{C}$ in the dark. Green FL was quantified via the FL1 channel (log scale) through a $525 \mathrm{~nm}$ band pass filter.

\subsection{Cell Cycle}

By using a cell-penetrating DNA-binding dye, Hoechst 33342, cellular DNA content could be measured without fixation and permeabilization. Briefly, cells were incubated with $20 \mu \mathrm{g} / \mathrm{mL}$ Hoechst 33342 in cell culture medium for $45 \mathrm{~min}$ at $37{ }^{\circ} \mathrm{C}$. The stained cells were analyzed by the 
Quanta SC MPL using the Hg arc lamp with a 355/37BP excitation filter as excitation and detected on the FL1 with a 465/30BP filter. Data acquisition was carried out by triggering on FL1.

\subsection{Electrophysiology}

For patch-clamp recordings, cells were allowed to attach for 3-4 h to $20 \mathrm{~mm}$ diameter glass cover slips. The electrophysiological studies were performed at room temperature $\left(23-25^{\circ} \mathrm{C}\right)$ on the stage of an inverted microscope (Nikon TE2000, Champigny-sur-Marne, France) using the whole-cell configuration of the patch-clamp technique in the voltage-clamp mode. Patch pipettes were pulled from borosilicate glass capillaries (Harvard Apparatus, Holliston, MA, USA) with a P-97 horizontal puller (Sutter Instrument, Novato, CA, USA). The intracellular (pipette) solution contained (in $\mathrm{mM}$ ): $134 \mathrm{CsCl}, 1.8 \mathrm{CaCl}_{2}, 1 \mathrm{MgCl}_{2}, 2$ ethylene glycol-bis(b-aminoethylether)$N, N, N^{\prime}, N^{\prime}$,-tetraacetic acid (EGTA), 10 4-(2-hydroxyethyl)piperazine-1-ethanesulfonic acid (HEPES) and 3 Na2ATP; adjusted to $\mathrm{pH} 7.2$ with $\mathrm{CsOH}$. The 20\%-hypotonic bath solution ( $240 \mathrm{mOsmol} / \mathrm{kg} \mathrm{H}_{2} \mathrm{O}$ ) contained (in $\mathrm{mM}$ ): 2.8 tetraethyl ammonium (TEA)-Cl, $100 \mathrm{~N}$-methyl-Dglucamine (NMDG)-Cl, $2 \mathrm{MgCl}_{2}, 1 \mathrm{CoCl}_{2}$ and $10 \mathrm{HEPES}$, adjusted to $\mathrm{pH} 7.4$ with $\mathrm{HCl}$. In order to avoid any change in $\mathrm{ECl}$, the osmolality was set at $300 \mathrm{mOsM}$ by adding mannitol. When in the bath and filled with the intracellular solution, patch pipettes had a resistance between 2 and $4 \mathrm{M} \Omega$.

Recordings were made with an Axopatch 200B patch-clamp amplifier interfaced to a $1.5 \mathrm{GHz}$ computer via a Digidata 1322 and pClamp 8.0 software (Axon instruments, Foster City, CA, USA). Cells with access resistances exceeding $10 \mathrm{M} \Omega$ immediately after gaining the whole cell configuration or during hypotonic challenge were discarded.

\subsection{Statistical Analysis}

All quantitative data were expressed as mean \pm standard error of the mean (SEM). Statistical analyses were performed with SigmaPlot 11 (Systat Software Inc., Chicago, IL, USA). A Shapiro-Wilk normality test, with a $p=0.05$ rejection value, was used to test normal distribution of data prior further analysis. All pairwise multiple comparisons were performed by one way ANOVA followed by Holm-Sidak posthoc tests for data with normal distribution or by Kruskal-Wallis analysis of variance on ranks followed by Tukey posthoc tests, in case of failed normality test. Paired comparisons were performed by Student's $t$-tests or by Mann-Whitney rank sum tests in case of unequal variance or failed normality test. Statistical significance was accepted for $p<0.05(*)$, $p<0.01(* *)$ or $p<0.001(* * *)$. All experiments were performed in triplicate.

For the purpose of $\mathrm{IC}_{50}$ value calculation (dose-response curves of calcein-AM assay), data were fitted to a sigmoidal three parameters dose-response model [58]:

$$
\mathrm{y}=\mathrm{b}+(\mathrm{a}-\mathrm{b}) /\left(1+10\left(\log \mathrm{IC}_{50}-\mathrm{x}\right)\right)
$$

where (y) is response, i.e., the ratio of mean FL1-FC in the presence of PSC833 to mean FL1-FC in control condition, (b) represents minimum of response, (a) represents maximum of response Emax, (x) is logarithm of PSC833 concentration and $\mathrm{IC}_{50}$ (or EC50) is the concentration of inhibitor that corresponds to $50 \%$ of maximal effect. 


\section{Conclusions}

In this study, we show evidence for a role of P-gp in cell volume regulation by combining microscopic observations, coulter volume, flow cytometry and electrophysiology. Taken together, our results strongly suggest that overexpression of P-gp in MCF7/Doxo cells has an impact on cell volume regulation in regular osmotic pressure and also during hypo-osmotic stresses. We highlight unexpected differences between two MCF-7 variants in regular osmotic conditions. Under osmotic challenge, P-gp overexpression influences cell volume regulation; it can then be supposed that P-gp overexpression contributes to chemoresistance not only by drug efflux, but also by volume stabilization and AVD-impairment. However, it should be emphasized that even if hypo-osmotic challenges associated with RVD monitoring remains an experimental approach to model AVD, a direct study of AVD seems necessary to validate our assumption. Overall, by multimodal cell volume monitoring, we were able to rekindle discussion on the role of P-gp in cell volume regulation.

\section{Acknowledgments}

The authors are indebted to Jean-Pierre Marie (Hôtel Dieu, Paris, France) for providing MCF7/Doxo cells, zosuquidar and PSC833. We would like to thank Guillaume Doubremelle Trébutien for his technical support.

This work was supported by European Regional Development Fund as part of the Interreg IVA Project Admin (Transchannel Advanced Microscopy network). Jennifer Pasquier and Damien Rioult were recipients for a fellowship from the Conseil Regional de Haute-Normandie.

This study was made possible by JSREP grant \#4-013-3-005 from the Qatar National Research Fund (a member of Qatar Foundation). The statements made herein are solely the responsibility of the authors.

\section{Author Contributions}

Jennifer Pasquier and Frank Le Foll planned experiments; Jennifer Pasquier and Damien Rioult performed experiments; Jennifer Pasquier, Damien Rioult, Jessica Hoarau-Véchot, Nadine Abu-Kaoud, Matthieu Marin and Frank Le Foll analyzed data; Matthieu Marin contributed reagents or other essential material; and Jennifer Pasquier, Jessica Hoarau-Véchot, Nadine Abu-Kaoud, Matthieu Marin and Frank Le Foll wrote the paper.

\section{Conflicts of Interest}

The authors declare no conflict of interest.

\section{Abbreviations}

ABC: ATP-binding cassette; AVD: apoptotic volume decrease; EV: electronic volume; MDR: MultiDrug Resistance; P-gp: P-glycoprotein; RVD: regulatory volume decrease; RVI: regulatory volume increase. 


\section{References}

1. Okada, Y. Volume expansion-sensing outward-rectifier $\mathrm{Cl}^{-}$channel: Fresh start to the molecular identity and volume sensor. Am. J. Physiol. 1997, 273, C755-C789.

2. Okada, Y.; Maeno, E. Apoptosis, cell volume regulation and volume-regulatory chloride channels. Comp. Biochem. Physiol. A Mol. Integr. Physiol. 2001, 130, 377-783.

3. Agre, P.; King, L.S.; Yasui, M.; Guggino, W.B.; Ottersen, O.P.; Fujiyoshi, Y.; Engel, A.; Nielsen, S. Aquaporin water channels-From atomic structure to clinical medicine. J. Physiol. 2002, 542 Pt 1, 3-16.

4. Sardini, A.; Amey, J.S.; Weylandt, K.H.; Nobles, M.; Valverde, M.A.; Higgins, C.F. Cell volume regulation and swelling-activated chloride channels. Biochim. Biophys. Acta 2003, 1618, $153-162$.

5. Lang, F.; Busch, G.L.; Volkl, H. The diversity of volume regulatory mechanisms. Cell. Physiol. Biochem. 1998, 8, 1-45.

6. Peak, M.; al-Habori, M.; Agius, L. Regulation of glycogen synthesis and glycolysis by insulin, $\mathrm{pH}$ and cell volume. Interactions between swelling and alkalinization in mediating the effects of insulin. Biochem. J. 1992, 282, 797-805.

7. Kroemer, G.; Galluzzi, L.; Vandenabeele, P.; Abrams, J.; Alnemri, E.S.; Baehrecke, E.H.; Blagosklonny, M.V.; El-Deiry, W.S.; Golstein, P.; Green, D.R.; et al. Classification of cell death: Recommendations of the Nomenclature Committee on Cell Death 2009. Cell Death Differ. 2009, 16, 3-11.

8. Okada, Y.; Maeno, E.; Shimizu, T.; Dezaki, K.; Wang, J.; Morishima, S. Receptor-mediated control of regulatory volume decrease (RVD) and apoptotic volume decrease (AVD). J. Physiol. 2001, 532, 3-16.

9. Bosch, I.; Croop, J. P-glycoprotein multidrug resistance and cancer. Biochim. Biophys. Acta 1996, 1288, F37-F54.

10. Endicott, J.A.; Ling, V. The biochemistry of P-glycoprotein-mediated multidrug resistance. Annu. Rev. Biochem. 1989, 58, 137-171.

11. Jentsch, T.J.; Gunther, W. Chloride channels: An emerging molecular picture. Bioessays 1997, 19, 117-126.

12. Nilius, B.; Eggermont, J.; Voets, T.; Droogmans, G. Volume-activated $\mathrm{Cl}^{-}$channels. Gen. Pharmacol. 1996, 27, 1131-1140.

13. Valverde, M.A.; Diaz, M.; Sepulveda, F.V.; Gill, D.R.; Hyde, S.C.; Higgins, C.F. Volume-regulated chloride channels associated with the human multidrug-resistance P-glycoprotein. Nature 1992, 355, 830-833.

14. Horton, J.K.; Vanoye, C.G.; Reuss, L. Swelling-activated chloride currents in a drug-sensitive cell line and a P-glycoprotein-expressing derivative are underlied by channels with the same pharmacological properties. Cell. Physiol. Biochem. 1998, 8, 246-260.

15. Hardy, S.P.; Goodfellow, H.R.; Valverde, M.A.; Gill, D.R.; Sepulveda, V.; Higgins, C.F. Protein kinase C-mediated phosphorylation of the human multidrug resistance P-glycoprotein regulates cell volume-activated chloride channels. EMBO J. 1995, 14, 68-75. 
16. Bond, T.D.; Valverde, M.A.; Higgins, C.F. Protein kinase C phosphorylation disengages human and mouse-1a P-glycoproteins from influencing the rate of activation of swelling-activated chloride currents. J. Physiol. 1998, 508 Pt 2, 333-340.

17. Ehring, G.R.; Osipchuk, Y.V.; Cahalan, M.D. Swelling-activated chloride channels in multidrug-sensitive and -resistant cells. J. Gen. Physiol. 1994, 104, 1129-1161.

18. Miwa, A.; Ueda, K.; Okada, Y. Protein kinase C-independent correlation between P-glycoprotein expression and volume sensitivity of $\mathrm{Cl}^{-}$channel. J. Membr. Biol. 1997, 157, 63-69.

19. De Greef, C.; Sehrer, J.; Viana, F.; van Acker, K.; Eggermont, J.; Mertens, L.; Raeymaekers, L.; Droogmans, G.; Nilius, B. Volume-activated chloride currents are not correlated with P-glycoprotein expression. Biochem. J. 1995, 307, 713-718.

20. De Greef, C.; van der Heyden, S.; Viana, F.; Eggermont, J.; de Bruijn, E.A.; Raeymaekers, L.; Droogmans, G.; Nilius, B. Lack of correlation between MDR-1 expression and volume-activation of cloride-currents in rat colon cancer cells. Pflugers Arch. 1995, 430, 296-298.

21. Marin, M.; Poret, A.; Maillet, G.; Leboulenger, F.; le Foll, F. Regulation of volume-sensitive $\mathrm{Cl}^{-}$channels in multi-drug resistant MCF7 cells. Biochem. Biophys. Res. Commun. 2005, 334, 1266-1278.

22. Poulsen, K.A.; Andersen, E.C.; Hansen, C.F.; Klausen, T.K.; Hougaard, C.; Lambert, I.H.; Hoffmann, E.K. Deregulation of apoptotic volume decrease and ionic movements in multidrug-resistant tumor cells: Role of chloride channels. Am. J. Physiol. Cell Physiol. 2010, 298, C14-C25.

23. Torre, A.; Trischitta, F.; Faggio, C. Purinergic receptors and regulatory volume decrease in seabream (Sparus aurata) hepatocytes: A videometric study. Fish Physiol. Biochem. 2012, 38, 1593-1600.

24. Pasquier, J.; Galas, L.; Boulange-Lecomte, C.; Rioult, D.; Bultelle, F.; Magal, P.; Webb, G.; le Foll, F. Different modalities of intercellular membrane exchanges mediate cell-to-cell p-glycoprotein transfers in MCF-7 breast cancer cells. J. Biol. Chem. 2012, 287, 7374-7387.

25. Pasquier, J.; Magal, P.; Boulange-Lecomte, C.; Webb, G.; Le Foll, F. Consequences of cell-to-cell P-glycoprotein transfer on acquired multidrug resistance in breast cancer: A cell population dynamics model. Biol. Direct. 2011, 6, 5.

26. Pasquier, J.; Rioult, D.; Abu-Kaoud, N.; Marie, S.; Rafii, A.; Guerrouahen, B.S.; le Foll, F. P-glycoprotein-activity measurements in multidrug resistant cell lines: Single-cell vs. single-well population fluorescence methods. Biomed. Res. Int. 2013, 2013, doi:10.1155/2013/676845.

27. Hille, B. Ion Channels of Excitable Membranes, 3rd ed.; Sinauer associates Inc.: Sunderland, MA, USA, 2001; p. 814.

28. Gill, D.R.; Hyde, S.C.; Higgins, C.F.; Valverde, M.A.; Mintenig, G.M.; Sepulveda, F.V. Separation of drug transport and chloride channel functions of the human multidrug resistance P-glycoprotein. Cell 1992, 71, 23-32. 
29. Sardini, A.; Mintenig, G.M.; Valverde, M.A.; Sepulveda, F.V.; Gill, D.R.; Hyde, S.C.; Higgins, C.F.; McNaughton, P.A. Drug efflux mediated by the human multidrug resistance P-glycoprotein is inhibited by cell swelling. J. Cell Sci. 1994, 107 Pt 12, 3281-3290.

30. Weaver, J.L.; Aszalos, A.; McKinney, L. MDR1/P-glycoprotein function. II. Effect of hypotonicity and inhibitors on $\mathrm{Cl}^{-}$efflux and volume regulation. Am. J. Physiol. 1996, 270, C1453-C1460.

31. Aleu, J.; Ivorra, I.; Lejarreta, M.; Gonzalez-Ros, J.M.; Morales, A.; Ferragut, J.A. Functional incorporation of P-glycoprotein into Xenopus oocyte plasma membrane fails to elicit a swelling-evoked conductance. Biochem. Biophys. Res. Commun. 1997, 237, 407-412.

32. Chen, L.X.; Wang, L.W.; Jacob, T. The role of MDRI gene in volume-activated chloride currents in pigmented ciliary epithelial cells. Sheng Li Xue Bao 2002, 54, 1-6.

33. Shi, X.; Wu, S.; Yang, Y.; Tang, L.; Wang, Y.; Dong, J.; Lu, B.; Jiang, G.; Zhao, W. AQP5 silencing suppresses p38 MAPK signaling and improves drug resistance in colon cancer cells. Tumour Biol. 2014, 35, 7035-7045.

34. Jablonski, E.; Webb, A.; Hughes, F.M., Jr. Water movement during apoptosis: A role for aquaporins in the apoptotic volume decrease (AVD). Adv. Exp. Med. Biol. 2004, 559, 179-188.

35. Jablonski, E.M.; Mattocks, M.A.; Sokolov, E.; Koniaris, L.G.; Hughes, F.M., Jr.; Fausto, N.; Pierce, R.H.; McKillop, I.H. Decreased aquaporin expression leads to increased resistance to apoptosis in hepatocellular carcinoma. Cancer Lett. 2007, 250, 36-46.

36. Hanahan, D.; Weinberg, R.A. The hallmarks of cancer. Cell 2000, 100, 57-70.

37. Johnstone, R.W.; Ruefli, A.A.; Tainton, K.M.; Smyth, M.J. A role for P-glycoprotein in regulating cell death. Leuk. Lymphoma 2000, 38, 1-11.

38. Yang, X.; Page, M. P-glycoprotein expression in ovarian cancer cell line following treatment with cisplatin. Oncol. Res. 1995, 7, 619-624.

39. Litman, T.; Nielsen, D.; Skovsgaard, T.; Zeuthen, T.; Stein, W.D. ATPase activity of P-glycoprotein related to emergence of drug resistance in Ehrlich ascites tumor cell lines. Biochim. Biophys. Acta 1997, 1361, 147-158.

40. Barakat, S.; Demeule, M.; Pilorget, A.; Regina, A.; Gingras, D.; Baggetto, L.G.; Beliveau, R. Modulation of P-glycoprotein function by caveolin-1 phosphorylation. J. Neurochem. 2007, $101,1-8$.

41. Barakat, S.; Turcotte, S.; Demeule, M.; Lachambre, M.P.; Regina, A.; Baggetto, L.G.; Beliveau, R. Regulation of brain endothelial cells migration and angiogenesis by P-glycoprotein/caveolin-1 interaction. Biochem. Biophys. Res. Commun. 2008, 372, 440-446.

42. Demeule, M.; Jodoin, J.; Gingras, D.; Beliveau, R. P-glycoprotein is localized in caveolae in resistant cells and in brain capillaries. FEBS Lett. 2000, 466, 219-224.

43. Pedersen, S.F.; Hoffmann, E.K.; Novak, I. Cell volume regulation in epithelial physiology and cancer. Front. Physiol. 2013, 4, 233.

44. Miyazaki, T.; Arai, S. Two distinct controls of mitotic cdk1/cyclin B1 activity requisite for cell growth prior to cell division. Cell Cycle 2007, 6, 1419-1425. 
45. Sveiczer, A.; Novak, B.; Mitchison, J.M. The size control of fission yeast revisited. J. Cell Sci. 1996, 109, 2947-2957.

46. Wells, W.A. Does size matter? J. Cell Biol. 2002, 158, 1156-1159.

47. Cooper, S. Control and maintenance of mammalian cell size. BMC Cell Biol. 2004, 5, doi:10.1186/1471-2121-5-35.

48. Jorgensen, P.; Tyers, M. How cells coordinate growth and division. Curr. Biol. 2004, 14, R1014-R1027.

49. Saucedo, L.J.; Edgar, B.A. Why size matters: Altering cell size. Curr. Opin. Genet. Dev. 2002, 12, 565-571.

50. Conlon, I.; Raff, M. Control and maintenance of mammalian cell size: Response. BMC Cell Biol. 2004, 5, doi:10.1186/1471-2121-5-36.

51. Umen, J.G. The elusive sizer. Curr. Opin. Cell Biol. 2005, 17, 435-441.

52. Ernest, N.J.; Habela, C.W.; Sontheimer, H. Cytoplasmic condensation is both necessary and sufficient to induce apoptotic cell death. J. Cell Sci. 2008, 121 Pt 3, 290-297.

53. Habela, C.W.; Sontheimer, H. Cytoplasmic volume condensation is an integral part of mitosis. Cell Cycle 2007, 6, 1613-1620.

54. Wang, H.; Vo, T.; Hajar, A.; Li, S.; Chen, X.; Parissenti, A.M.; Brindley, D.N.; Wang, Z. Multiple mechanisms underlying acquired resistance to taxanes in selected docetaxel-resistant MCF-7 breast cancer cells. BMC Cancer 2014, 14, 37.

55. Murray, S.; Briasoulis, E.; Linardou, H.; Bafaloukos, D.; Papadimitriou, C. Taxane resistance in breast cancer: Mechanisms, predictive biomarkers and circumvention strategies. Cancer Treat. Rev. 2012, 38, 890-903.

56. Maulard, C.; Marie, J.P.; Delanian, S.; Housset, M. Clinical effects on CA 15-3 level of cyclosporin $\mathrm{A}$ as a chemosensitiser in chemoresistant metastatic breast cancer. Eur. J. Cancer 1993, 29A, 480.

57. Le Foll, F.; Rioult, D.; Boussa, S.; Pasquier, J.; Dagher, Z.; Leboulenger, F. Characterisation of Mytilus edulis hemocyte subpopulations by single cell time-lapse motility imaging. Fish Shellfish Immunol. 2010, 28, 372-386.

58. Rioult, D.; Pasquier, J.; Boulange-Lecomte, C.; Poret, A.; Abbas, I.; Marin, M.; Minier, C.; le Foll, F. The multi-xenobiotic resistance (MXR) efflux activity in hemocytes of Mytilus edulis is mediated by an ATP binding cassette transporter of class C (ABCC) principally inducible in eosinophilic granulocytes. Aquat. Toxicol. 2014, 153, 98-109. 


\title{
A Review of Cell Adhesion Studies for Biomedical and Biological Applications
}

\section{Amelia Ahmad Khalili and Mohd Ridzuan Ahmad}

\begin{abstract}
Cell adhesion is essential in cell communication and regulation, and is of fundamental importance in the development and maintenance of tissues. The mechanical interactions between a cell and its extracellular matrix (ECM) can influence and control cell behavior and function. The essential function of cell adhesion has created tremendous interests in developing methods for measuring and studying cell adhesion properties. The study of cell adhesion could be categorized into cell adhesion attachment and detachment events. The study of cell adhesion has been widely explored via both events for many important purposes in cellular biology, biomedical, and engineering fields. Cell adhesion attachment and detachment events could be further grouped into the cell population and single cell approach. Various techniques to measure cell adhesion have been applied to many fields of study in order to gain understanding of cell signaling pathways, biomaterial studies for implantable sensors, artificial bone and tooth replacement, the development of tissue-on-a-chip and organ-on-a-chip in tissue engineering, the effects of biochemical treatments and environmental stimuli to the cell adhesion, the potential of drug treatments, cancer metastasis study, and the determination of the adhesion properties of normal and cancerous cells. This review discussed the overview of the available methods to study cell adhesion through attachment and detachment events.
\end{abstract}

Reprinted from Int. J. Mol. Sci. Cite as: Khalili, A.A.; Ahmad, M.R. A Review of Cell Adhesion Studies for Biomedical and Biological Applications. Int. J. Mol. Sci. 2015, 16, 18149-18184.

\section{Introduction}

Adhesion plays an integral role in cell communication and regulation, and is of fundamental importance in the development and maintenance of tissues. Cell adhesion is the ability of a single cell to stick to another cell or an extracellular matrix (ECM). It is important to understand how cells interact and coordinate their behavior in multicellular organisms. In vitro, most mammalian cells are anchorage-dependent and attach firmly to the substrate [1]. According to the "cell adhesion model", the more a cell sticks the more it shows the greater number of chemical bonds it has on its surface [2,3].

Cell adhesion is involved in stimulating signals that regulate cell differentiation, cell cycle, cell migration, and cell survival [4]. The affinity of cells to substrate is a crucial consideration in biomaterial design and development. Cell adhesion is also essential in cell communication and regulation, and becomes of fundamental importance in the development and maintenance of tissues. Changes in cell adhesion can be the defining event in a wide range of diseases including arthritis $[5,6]$, cancer $[4,7,8]$, osteoporosis $[9,10]$, and atherosclerosis $[11,12]$. Cell adhesiveness is generally reduced in human cancers. Reduced intercellular adhesiveness allows cancer cells to disobey the social order, resulting in destruction of histological structure, which is the morphological 
hallmark of malignant tumors [8]. Tumor cells are characterized by changes in adhesivity to ECM, which may be related to the invasive and metastatic potential. Alterations in cell-matrix and cell-cell interactions are cell type- and oncogene-specific. For example, while the transfection of rodent fibroblast cells with $\operatorname{Src}$ and Ras oncogenes reduces the adhesiveness to fibronectin (Fn) by impairing $\alpha 5 \beta 1$ integrins, the activation of oncogene $E r b B 2$ in breast cancer up-regulates $\alpha 5 \beta 1$ integrin and enhances adhesion [13,14]. The adhesion of highly invasive cancer cells altered the biomechanics of endothelial cells [15]. Mierke [15] reported that $M D A-M B-231$ cells' attachment may lower the endothelial cells' stiffness by breaking down the cells' barrier function through remodelling of the actin cytoskeleton.

Different requirements for cell adhesion are needed for various types of applications, and are dependent on the cell's specific applications [16]. Various techniques to analyze cell adhesion have been applied to understand different fields of study including biomaterial studies [17], the effects of biochemical treatments and environmental stimuli to the cell culture [18], and determination of adhesion properties of normal and cancerous cells [19]. Biomaterials designed in biomedical engineering that have to interact with blood, like those in artificial heart valves or blood vessels, are required not to be adherent to cells or plasma proteins to avoid thrombosis and embolism. On the other hand, materials used in scaffolds for tissue generation are needed to act as substrate to promote the cells' adhesion, subsequent proliferation, and biosynthesis [16]. Adhesion between cells allows blood clot formations that may lead to heart failure by restricting the blood supply to the heart muscles [16].

\subsection{Focal Adhesion}

Cells transmit extracellular or intracellular forces through localized sites at which they are adhered to other cells or an extracellular matrix. The adhesion sites are formed by transmembrane proteins called integrins to anchor the cell to a matrix or adhesion molecules to other cells [20]. Both the integrins and adhesion molecules are attached to the tensile members of the cytoskeleton, the actin filaments, through the focal adhesion (FA) complex (Figure 1), a highly organized cluster of molecules [21]. The cytoskeletal structure holds the nucleus and maintains the shape of the cell [22-24]. As a pathway for force transmission to the cytoskeleton, integrins play an important role in mechanotransduction through FA proteins connecting the integrin domains to the actin filaments to form the adhesion complex [24]. Upon binding, integrins cluster into FA complexes that transmit adhesive and traction forces $[25,26]$. The FA formation is important in cell signaling to direct cell migration [27], proliferation, and differentiation [28,29] for tissue organization, maintenance, and repair [28]. 


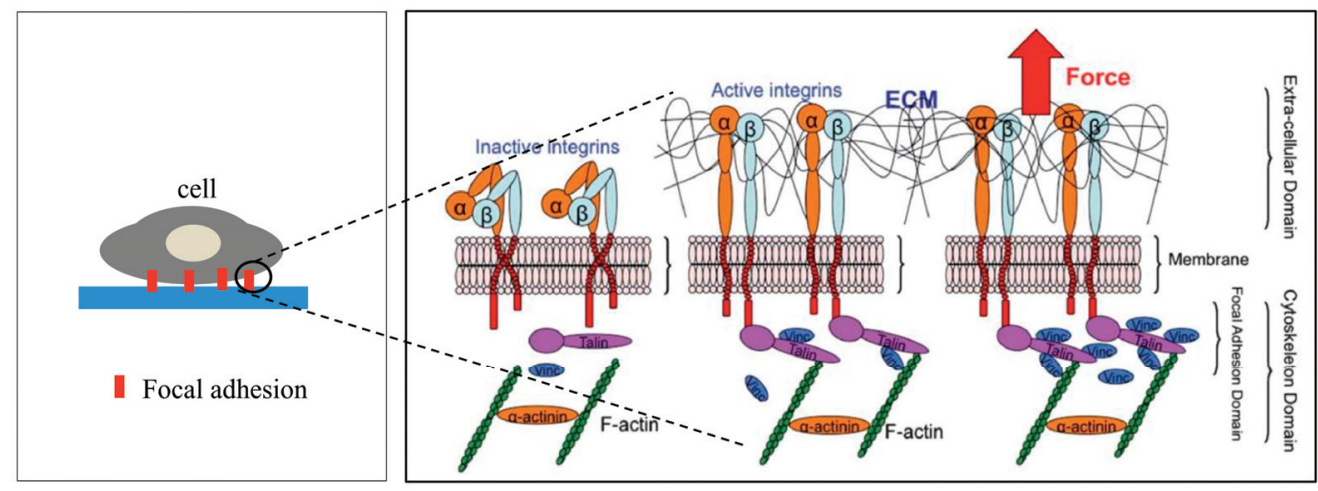

Figure 1. Schematic representation of activated integrin and formation of ECM-integrin-cytoskeleton linkages in the focal adhesion site upon application of an external tensile load. Reproduced "in part" from [24] with permission of The Royal Society of Chemistry.

\subsection{Phases of Cell Adhesion and Spreading}

\subsubsection{Passive in Vitro Cell Adhesion}

Passive in vitro cell adhesion is the cell adhesion process in a static medium culture, e.g., culture flasks, petri dishes. During static in vitro cell-matrix attachment and spreading, cells undergo morphologic alterations driven by passive deformation and active reorganization of the cytoskeleton. Integrin receptors and heterodimeric transmembrane proteins play a central role in cell adhesion and spreading. Specific integrin binding provides not only a mechanical linkage between the intracellular actin cytoskeleton and ECM, but also the bidirectional transmembrane signaling pathways [29-33]. Integrins recognize soluble ligands and insoluble ECM proteins and their interaction regulates cell responses such as cytoskeleton formation. The binding of integrins with their ECM proteins activates the Rho GTPase family (including Rho, Rac, and Cdc42), which is involved in cell spreading and migration [34,35], and Rho controls stress fiber formation and the assembly of focal adhesions [34].

The process of static in vitro cell adhesion is characterized by three stages (Table 1): attachment of the cell body to its substrate (initial stage), flattening and spreading of the cell body, and the organization of the actin skeleton with the formation of focal adhesion between the cell and its substrate [35]. Cell spreading appears to be accompanied by the organization of actin into microfilament bundles. The strength of adhesion becomes stronger with the length of time a cell is allowed to adhere to a substrate or another cell. The initial adhesive interaction between the cells and the substrate are driven by the specific integrin-mediated adhesion and starts with the binding of single receptor-ligand pairs [36]. This will initiate the subsequent receptor-ligand bonds and quickly enhance in number, thus increasing the total adhesion strength [37]. The adhesion properties of cells could be determined by studying various cell-substrate contact times [38]. 
Following initial attachment, cells continue flattening and spreading on the substrate, resulting in the decrement of cell height (the cell flattens) and increment of contact area (Phase I) [16]. Next, the cell spreads beyond the projected area of the unspread spherical cell (Phase II) [36]. The spreading process is the combination of continuing adhesion with the reorganization and distribution of the actin skeleton around the cell's body edge [16]. Cells will reach their maximum spread area through expansion and adhesion strength will become stronger (Phase III).

Table 1. Evaluation of passive in vitro cell adhesion intervention and stages [36].

\begin{tabular}{|c|c|c|c|}
\hline $\begin{array}{c}\text { Cell Adhesion } \\
\text { Phases }\end{array}$ & Phase I & Phase II & Phase III \\
\hline $\begin{array}{l}\text { Schematic diagram } \\
\text { of cell adhesion }\end{array}$ & 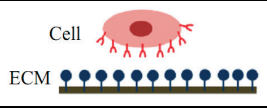 & 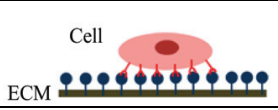 & ECM \\
\hline $\begin{array}{c}\text { Schematic diagram } \\
\text { of the transformation } \\
\text { of cell shape }\end{array}$ & Initial attachment & Flattening & $\begin{array}{l}\text { Fully spreading and structural } \\
\text { organization }\end{array}$ \\
\hline $\begin{array}{c}\text { Cell adhesion } \\
\text { intervension }\end{array}$ & $\begin{array}{l}\text { Electrostatic } \\
\text { interaction }\end{array}$ & Integrin bonding & Focal adhesion \\
\hline Adhesion stages & Sedimentation & Cell attachment & Cell spreading and stable adhesion \\
\hline
\end{tabular}

\subsubsection{Dynamic in Vivo Cell Adhesion}

The adhesion of cells to the extracellular matrix in vivo under blood flow is a dynamic process. Cells will undergo dynamic adhesion alterations during their morphogenesis, tissue remodeling, and other responses to environmental cues [39]. In vivo dynamic cell adhesion is mediated through molecular bonding between cell-surface receptors and their ligands or counter-receptors on the other cell surfaces in the extracellular matrix. Shear flow is a crucial factor to initiate cell adhesion as it mediates the activation of $\beta$-integrin via E-selectin signaling [40]. The adhesive bond is defined as the sum of non-covalent interactions, e.g., hydrogen bonds, electrostatic interactions, van der Waals forces, dipole-dipole interactions between two macro molecules [39]. Leukocytes or hematopoietic progenitors and tumor cells are the cells involved in dynamic vascular cell adhesion in vivo. Leukocytes and hematopoietic progenitors are the essential cells in the human immune response system that will migrate from one site to another to provide the effector function. Tumor cell interactions with endothelium and the subendothelial matrix constitutes a crucial factor in determining the metastatic potential of the cells and organ preferences of cancer metastasis $[41,42]$.

The cell adhesion cascade and signaling events in vivo involve three basic steps: selectin-mediated rolling, chemokine-triggered activation, and integrin-dependent arrest [43]. Initial recognition of dynamic cell adhesion in vivo involves the "docking" phase, which occurs between the rolling of cells to endothelial cells and to cell arrest (Figure 2), mediated by a weak and transient adhesion mechanism involving carbohydrate-carbohydrate and/or carbohydrate-protein interactions. The molecules involved at this stage are cell-surface conjugates, selectins, chemokines, or immunoglobulins (Igs) [42]. The cell adhesion cascade begins as a cell tethers to roll on the vessel's wall. Molecular bonding between the adhesion molecules must form rapidly for cells to 
tether, and the bonding must break rapidly for cells to roll [39]. The rolling cells transduce signals from adhesion receptors and chemokine receptors that cause cells to roll slower and then to arrest, a prerequisite for emigration through the vasculature into underlying tissue [39].

Subsequently, cells will established stable bonds with endothelial cells during the activationdependent "locking" phase, mediated largely by integrins and modulated by a host of bioactive mediators resulting from the activated cells [42]. Integrin-mediated adhesion is characterized by at least two events: arrest from rolling, which is mediated by increased cell avidity to endothelium, and a post-binding phase of adhesion stabilization [43]. In the "locking" phase, cell adhesion strengthening and spreading happens similarly to the static in vitro adhesion followed by intravascular crawling and transmigration (paracellular or transcellular) (Figure 2) [43]. This then permits the adhered cells to emigrate out of the vasculature. The intraluminal crawling facilitates the cell adhesion to emigration [44]. The post-adhesion events strengthen cell attachment to the endothelium, and into the molecules that are involved in cells' transendothelial migration $[45,46]$.
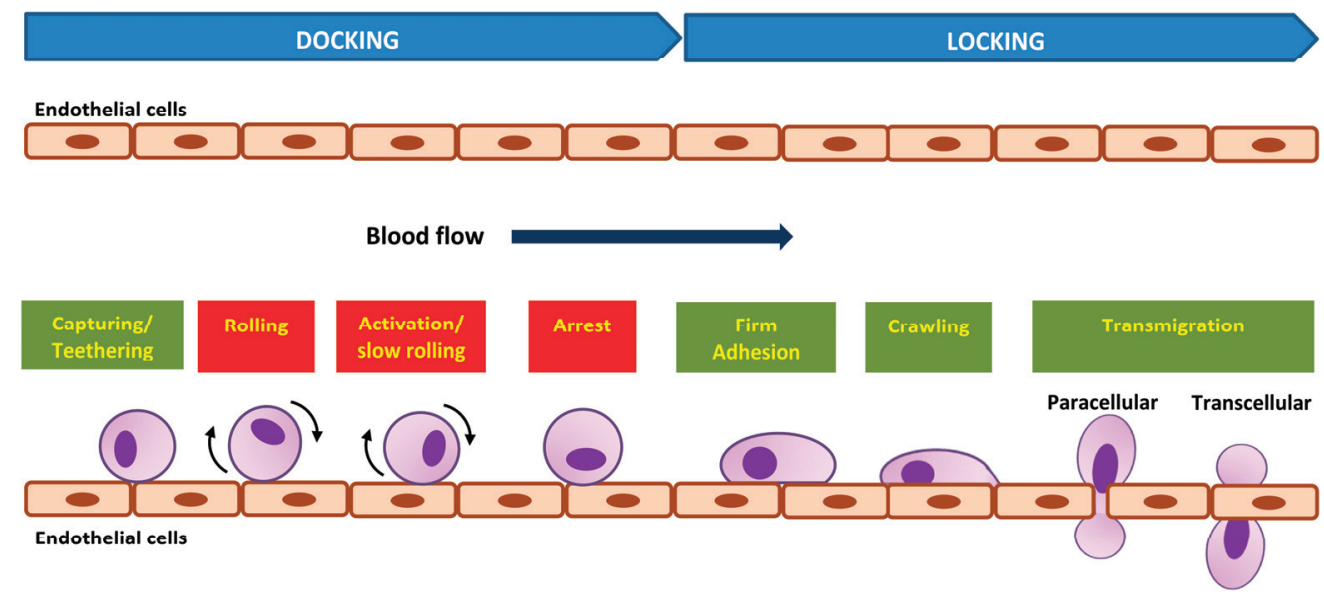

Figure 2. Dynamic in vivo cell adhesion cascade with "docking" and "locking" phases. The basic cascade steps are labeled in red boxes and steps recovered later are labeled in green boxes.

\section{Types of Adhesion Studies}

The mechanical interactions between a cell and its ECM can influence and control cell behavior and function. The essential function of cell mechanobiology and its progressively important role in physiology and disease have created tremendous interests in developing methods for measuring the mechanical properties of cells. In general, cell adhesion studies can be categorized into cell attachment and detachment events. Numerous techniques have been developed to analyze cell adhesion events through the study of single cells as well as the populations of cells. Cell adhesion attachment events are focusing on the cell attachment mechanism to the substrate, while the detachment events involve the application of load to detach the adhered cells on the substrate (Figures 3 and 4). 


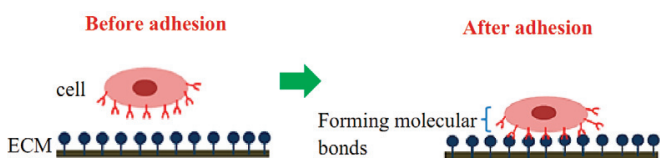

(a)

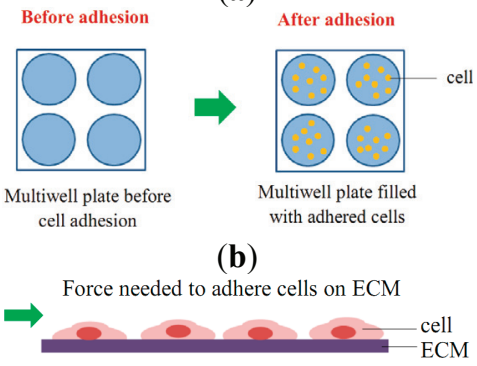

(c)

Figure 3. Schematic diagram of cell adhesion attachment events for (a) single cell studies via the formation of molecular bonds; (b) cell population studies via static adhesion (e.g., wash assay technique); and (c) cell population studies via dynamic adhesion (e.g., microfluidic technique).

\subsection{Cell Adhesion Attachment Events}

Cell attachment studies cover the analysis from the formation of a molecular bond between the cell's surface receptors and the complementary ligands (on the ECM's surface) to the observation of a population of cells' responses through the cells' behavior and changes of morphology during the attachment events. In cell migration, the cell adhesion plays a pivotal role in the driving force production [27]. The adhesion events could be grouped into single cell and cell population analysis (Figure 5). For the single cell study, the experiments were performed to analyze the interaction forces between the individual cell and its substrate (Figure 3a), observing the individual cell's morphology changes, studying the cell's migration, and measuring the cell's traction forces using polyacrylamide (PA) gel-based traction force microscopy (PA-TFM), micropattering technique, and three-dimensional traction force quantification (3D-TFM). Population studies involve the analysis of attachment events for a group of cells. It is important in analyzing the adhesion behavior of cells toward treatments or different physiological conditions (e.g., wash assay and microfluidic techniques), for the understanding the cell adhesion kinetics (e.g., resonance frequency technique), in determining the biocompatibility of biomaterials for tissue engineering, cancer metastasis studies, and also the potential of drug treatments (e.g., microfluidic techniques).

\subsubsection{Techniques to Study Cell Attachment Events}

Attachment Events: Single Cell Approach

Polyacrylamide-Traction Force Microscopy (PA-TFM). Numerous techniques have been developed to understand cell adhesion by characterizing single cells during their attachment events. 
PA-TFM is one of the widely used techniques to study single cells' traction force, the force exerted by cells through contact to the substrate surface. Cells will be cultured on the polyacrylamide gel functionalized with the cells' adhesive ligands and fluorescent beads embedded near the gel surface [47]. Upon the adhesion attachment events, cells will generate traction forces that move the beads and the movement will be quantified by tracking the displacement of the fluorescent beads [47-49]. Reinhart-King et al. [50] reported cell spreading increased with the increasing concentration of arginine-glycine-aspartic acid (RGD)-peptide by measuring the magnitude, direction, and spatial location of mechanical forces exerted by endothelial cells. Sabass et al. [51] improved the reliability and spatial resolution of traction force microscopy to $1 \mathrm{um}$ by combining the advances of experimental computational methods. An epithelial wound-healing assay was developed by $\mathrm{Ng}$ et al. [52] to study the migration of individual MCF10A on PA substrates with a range of substrate compliances. Their findings showed that the wound could initiate a wave of motion that directs cells' coordination towards the wound edge and substrate stiffness influenced the collective cell migration speed, persistence, and directionality as well as the coordination of cell movements [52]. Traction forces of human metastatic breast, prostate, and lung cancer cell lines were found to be higher than non-metastatic counterparts, suggesting the cellular contractile force involve in metastasis and the physiological environment might regulate cellular force generation [48]. Wen et al. [53] reported stem cell differentiation was regulated by the stiffness of planar matrices independently of protein tethering and porosity. Beside single cell analysis, there have also been some adhesion studies done on the population of cells. Endothelial cells were reported to exert greater traction forces compared to single cells once in contact with the adjacent cell, thus suggesting an increase in cellular contractility with contact [54].

Micropatterning. Micropatterning (also known as microfabricated elastomeric post array (mPADs) or micropillar) is a method that provides a micrometer scale: a soft, three-dimensional complex and dynamic microenvironment for both single cell studies and also for the multi-cellular arrangements in populations of cells. It relies on basic elastic beam theory, which makes force quantification easier and more reliable, as there is only one traction force field for each micropost/micropillar displacement map [55]. Cell micropatterning comprises the fabrication and use of a culture substrate with microscopic features that impose a defined cell adhesion pattern. It is an efficient method to investigate the sensitivity and response of a cell to specific microenvironmental cues [56]. At the basic level, micropatterning approaches involve controlling cellular attachment, shape, and spreading as a function of the engineered spatial properties of the cultured surface [57]. Micropattering could be used to study cell adhesion for both the single cell level and also for the population of cells. Tan et al. [26] found that cell morphology regulates the magnitude of traction force generated by cells. These findings demonstrate a coordination of biochemical and mechanical signals to regulate cell adhesion and mechanics, which introduce the use of arrays of mechanically isolated sensors to manipulate and measure the mechanical interactions of cells [26]. Mandal et al. [58] introduced the micropatterned surfaces combined with the thermo-responsive poly( $N$-isopropyla-crylamide) (PNIPAM) as an actuator which induces cell detachment when the temperature is reduced below $32{ }^{\circ} \mathrm{C}$. It has been reported that the micropatterning technique is able to independently tune the biochemical, mechanical, and 
spatial/topography properties of biomaterials that could provide the opportunity to control cell fate for tissue engineering and regenerative medicine applications [59]. Laminar atheroprotective fluid shear stress has been found to induce increments in traction force generation and endothelial cell alignment, which are associated with inflammation and atherosclerosis progression [60].

Three-Dimensional Traction Force Quantification (3D-TFM). The ability to grow cells within ECM gels (3D culture) is a major advantage to understand in vivo cellular cell behaviors, ranging from differentiated function to maintenance of stem cell functions [61,62]. The 3D-TFM technique uses 3D matrixes such as agarose, collagen, hyaluronic acid, fibrin, or matrigel for the cell culture. Individual cells are grown inside the gel matrix embedded with fluorescent beads surrounding the cell. Bead dispersion in the 3D gel will be observed to estimate the cellular contractility of the cell during migration [63-67]. In contrast to $2 \mathrm{D}$ migration, cell migration through a dense 3D network of extracellular matrix proteins is possible only when the cell generates sufficient tractions to overcome the steric hindrance of the surroundings [68]. Koch et al. [69] used the method to develop the 3D traction map of several tumor cell lines ( $M D A-M B-231$ breast carcinoma, A-125 lung carcinoma) and found that the directionality is important for cancer cell invasion rather than the magnitude of traction, and the invasive cells elongated with spindle-like morphology as opposed to the more spherical shape of non-invasive cells [69]. The disruptive effect of the nocodazole drug on the neuronal processes has been analyzed using matrigel-embeded microbeads and neuron cells [65]. Kutys and Yamada [70] managed to explored pathways controlling the migration involving the GEF/GAP interaction of $\beta P i x$ with $\operatorname{srGAPl}$ that is critical for maintaining suppressive crosstalk between $C d c 42$ and RhoA during 3D collagen migration. Fraley et al. [66] reported that instead of forming aggregates in the 3D matrix, the focal adhesion proteins diffused and distributed throughout the cytoplasm and were responsible in modulating cell motility.

\section{Attachment Events: Population Approach}

Wash Assay. In the population cell adhesion studies, the process of cell attachment can be divided into two types; static culture and dynamic culture, depending on the cell adherence mechanism during the cell culturing. Static culture is the stagnant condition of the cell culture medium during the incubation for cell adhesion, which is applicable for the culturing of cells inside microwell plates (Figure 3b), petri dishes, culture flasks, and cell cultures on the ECM-coated cantilever inside the chamber. The static culture was used in the wash assay and resonance frequency techniques. In the wash assay, cells were cultured in 96 multiwell plates for the cell attachment events, followed by cell washing before adhesion analysis (e.g., cell count, protein/DNA count, or antibody binding) was carried out [71-74]. Wash assays provide basic qualitative adhesion data by determining the fraction of cells which remain adhered after one or more washings [75]. Cells that remain adhered to the substrate after washing will be analyzed for further quantitative analysis such as cell count, quantification of DNA content, protein count, or antibody binding. Treatment of $D$. mucronata crude extract to the cancerous wehi-164 cells significantly modulated their attachment and spreading behavior to the fibronectin-coated multiwell plates [74]. Chen et al. [73] extracted the adhered HeLa cells from the collagen-coated multiwell for further 
enrichment process and analysis. Park et al. [71] developed adhesion-based assay for highthroughput screening for the discovery of small-molecule regulators of the integrin CD11b/CD18 to further understand the mechanism of integrin activation and binding.

Resonance Frequency. The integration of advanced microelectronics technology with signaling processing and biological sensing interfaces has grown widely to develop biosensor devices. Piezoelectric sensor is the acoustic sensor which is able to detect label-free and selective biological events in real time. Quartz crystal microbalance (QCM) is one of the widely used piezoelectric acoustic wave resonator [76] biosensors for the study of cell adhesion and cell spreading. It is comprised of a thickness shear mode resonator made from a thin (AT-cut) quartz crystal sandwiched between two metal electrodes [77]. The sensor is coated with ECM before cells are mounted on it and placed in the chamber for cell adhesion to occur. Whole cells will act as sensing elements in the cell-based biosensors, as cells continuously react to the environment. The piezoelectric resonators will perform shear oscillations parallel to the sensor faces [78] upon cell adhesion activity, which will propagate through the sensor in a direction perpendicular to its surface. During attachment and cell spreading on the sensor surface, changes in resonant frequency could be detected upon the interactions between the cell membrane and the substrate, and upon the changes in the fractional surface coverage by the cells [77-85]. The resonance frequency of the sensor alters when a foreign mass attaches to the sensor's active surface and the frequency shifts will represent the mass of the absorbed material [77]. The adhesion process and the molecular interactions will produce signals representing cell adhesion kinetics determined by the sensor $[36,83,86,87]$. This technique has been found able to monitor cell attachment and spreading of animal cells on a particular surface quantitatively and in real time.

Zhu et al. [87] found the mechanisms governing elasticity and adhesion are coupled and affected differently during aging. Heitmann and Wegener [78] reported that the resonance frequency technique not only can be used to monitor cell adhesion but can also present as an actuator to perturb cell-substrate contact sites without causing damage to the cell when using the driving voltage for normal monitoring amplitude. The propagation properties of the acoustic wave in materials vary according to the different properties of the materials, and their energy is dissipated in the presence of fluids or viscoelastic materials $[76,88]$. The variation of the sensor resonance frequency $(\Delta f)$ and acoustic wave energy dissipation can be used to gain direct measurements of the physical properties of the layers in contact with the chip [76,88,89]. The obvious difference in total frequency shift is caused by the different numbers of attached cells, since the geometries and the fundamental resonance frequency are similar. Besenbacher et al. [84,85] analyzed cell adhesion and spreading of MC3T3-E1 and NIH3T3 cells on different precoated biocompatible surfaces. Braunhut et al. [90-93] have developed and optimized the performance of the QCM biosensor and carried out a study on the effects of various types of anti-tumor drugs (e.g., nocodazole, taxol, taxane) on the adhesion of different types of cancer cells. In the advancement of resonance frequency technology, the sensor has been shown capable of acting as both sensor and actuator. It can be used as a sensor for cell adhesion and as an actuator to induce oscillations on the growth surface [78]. Recently, the usage of resonance frequency technique in cell adhesion has emerged to provide a platform for small sample sizes of cells in a dynamic fluid condition. Resonance 
frequency technique is used as an actuator to provide the acoustic wave needed for the dynamic fluid condition in the device and as sensor to measure cell adhesion. Warrick et al. [94] have developed a high-content adhesion assay to overcome the limitation of the available methods to perform analysis on small animal biopsies and rare cell isolations. Hartmann et al. [95] produced a new tool for the dynamic analysis of cell adhesion that provides small cells' sample volume, a short measuring time, and flexibility for different types of substrates that are suitable for studying the implant material compatibility.

Microfluidics. In contrast to the static culture, the dynamic culture applies fluid movement during the cell culturing and adhesion process. Low fluid shear flow is needed to help the cell attachment process as it mimics the blood flow in the human body. Cells are continuously exposed to hemodynamic forces generated by blood flow in most biological systems. The balance between the adhesive forces generated by the interactions of membrane-bound receptors and their ligands with the dispersive hydrodynamic forces determines cell adhesion [96]. Cell adhesion attachment events dynamic culture can be observed using microfluidic technique. The advantages of microfluidic systems (fluid manipulation and control, low fluid intake and miniaturization) encouraged their use in dynamic culturing for cell adhesion studies. This technique was used to study the ability of cells to adhere and to observe cell spreading, tracking, and migration inside the channel under the influence of fluid flow. Rupprecht et al. [97] reported that cell shape, movements, and the rate of cell division were found to be similar in petri dishes and microfluidic channels. Alapan et al. [98] applied the microfluidic technique to analyze the adhesion properties and deformability of human red blood cells in flow using blood samples from 12 different subjects. From the study of cell adhesion properties and dynamics, the technology has grown and been upgraded into the development of tissue-on-a-chip and, later, organ-on-a-chip for biomedical and pathological studies. Cell monolayers were grown in the microfluidic channel and made to mimic the human vascular system to be used for important bioengineering and biomedical analysis. A multi-step microfluidic device has been developed by Chaw et al. (2007) to analyze the deformation and biological and migratory capability of various tumor cell lines (HepG2, HeLa, and $M D A-M B 435 S$ ) to the lining of HMEC cells inside the channels [99]. Nalayanda et al. [100] have built a series of bio-mimetic devices for the model of alveolar-capillary membranes while Song et al. [101] have developed a cancer cell metastasis model using microfluidic technology. $\mathrm{Fu}$ et al. [102] studied tumor cell adhesion to the endothelium cell layer under shear flow by combining micro-particle imaging velocimetry ( $\mu \mathrm{PIV}$ ) technique with flow chamber assay to understand the interactions between leukocytes and tumor cells near the endothelium wall region. A microfluidic model for organ-specific extravasation of circulating tumor cells (CTCs) has been build by Riahi et al. [103] to demonstrate the extravasation of $M D A-M B-231$ cancer cells by analyzing the cells' adhesion capability to the endothelial monolayer inside the channel.

\subsection{Cell Adhesion Detachment Events}

Cell adhesion detachment studies involve load application to the adhered cells on the ECM to free the cells from their cell-matrix bonding (Figure 4). The applied force that produces cell detachment is quantified as the cell's adhesion strength. Many types of assays have been developed 
to measure cells' adhesion strengths and can be divided into single cell and population cell studies (Figure 4b,c). Measuring cells' adhesion strengths has become an emerging interest in various areas of study, including biomaterial compatibility in the human body, characterizing different stages of cancer cells, drug treatments for diseases, and the discovery of biomarkers for early disease diagnosis. Cells will be cultured and allowed to adhere on the ECM-coated matrix followed by cell detachment processes and adhesion strength measurement. For the single cell approach, the detachment process is focused on an individual cell and the measured value represents the adhesion strength of a single cell. Single cell detachment techniques were carried out either to fully detach a single cell from its substrate (whole cell detachment) for obtaining a single cell's adhesion strength or to focus on the load needed for breaking the molecular bonds to further understand cell adhesion kinetics. Techniques used for whole cell detachment are the cytodetachment and micropipette aspiration techniques, while to perform molecular bond breakage, the single cell force spectroscopy (SCFS) technique was used (Figure 4). Cell detachment events for the cell population approach were carried out by applying load at the population on adhered cells. Following the detachment process, the fraction of cells remaining on the substrate is quantified after varying loads of global force or stress have been applied. The force or stress value at which $50 \%$ of the cells detach is determined as the population adhesion strength. The adhesion measurement techniques for the cell population approach can be divided into four categories depending on the loading method applied to detach cells: centrifugation assay, spinning disk, flow chamber, and microfluidics (Figure 5).

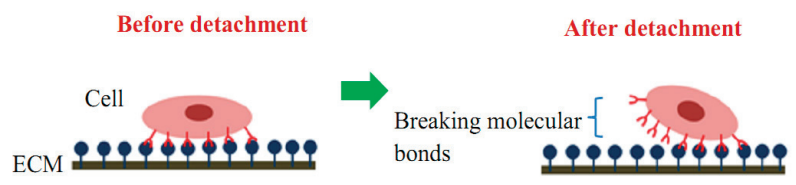

(a)

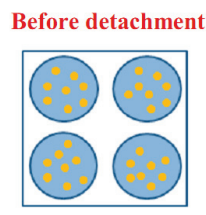

Multiwell plate filled with adhered cells

Force needed to detach

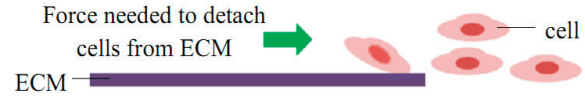

(c)

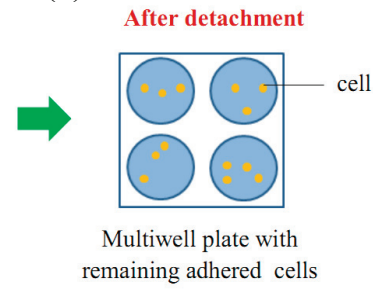

(b)

Figure 4. Schematic diagram of cell adhesion detachment events for (a) single cell studies via the breakage of molecular bonds (e.g., SCFS, micropipette aspiration, and optical tweezer techniques); (b) cell population studies via static adhesion (e.g., centrifugation technique); and (c) cell population studies via dynamic adhesion (e.g., spinning disk, flow chamber, and microfluidic techniques). 


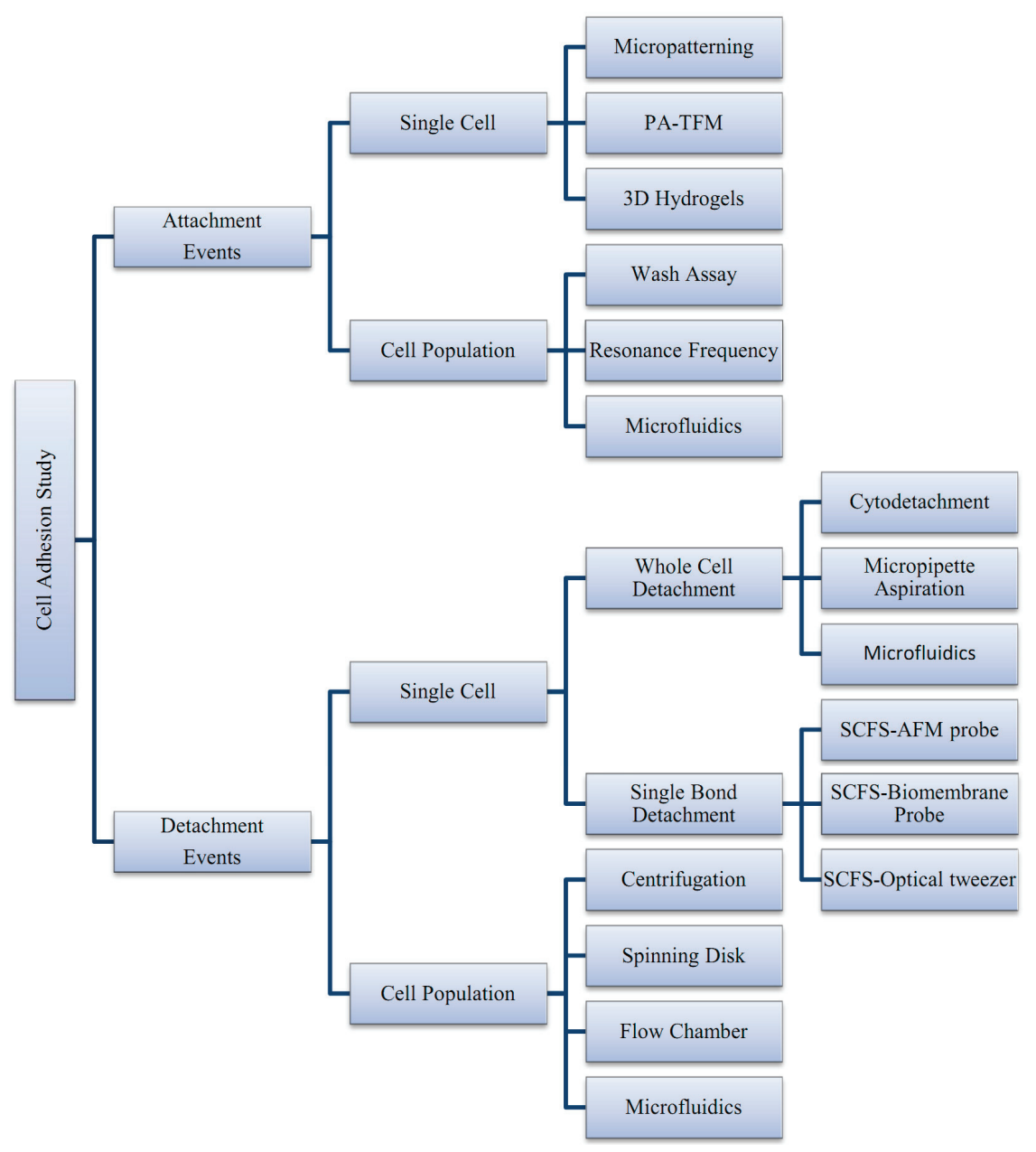

Figure 5. Summary of techniques involved in cell adhesion studies, categorized by the adhesion attachment events and detachment events.

\subsubsection{Techniques to Study Cell Detachment Events}

Single Cell Approach

Cytodetachment. Cytodetachment technique uses an atomic force microscopy (AFM) probe to physically detach individual cells in an open medium environment such as petri dish [75]. Cells have to be attached to a functionalized matrix before the single cell-probe alignment and probe translation application are carried out to detach the cell. Force is quantified by measuring the elastic deflection of the probe used to detach the cell and then divided by the cell area to calculate the average shear stress for each cell [75]. Yamamoto et al. [104] studied the force needed to detach murine fibroblast $L 929$ on four different materials by using cytodetachment technique. Using an image analysis system and fiber optic sensor, the apparent cell adhesive area was measured and the 
adhesive strength and detachment surface energy were calculated by dividing them by the cell adhesive area [104]. Their findings showed that cells on collagen-coated polystyrene produce the highest adhesion strength and cell detachment surface energy, and the adhesive properties of the cells between both polystyrene and glass are almost the same [104]. Human cervical carcinoma cells (NHIK 3025) were found to attached stonger and faster to the hydrophilic substrate using technique 1 at $37{ }^{\circ} \mathrm{C}$ when compared to $23{ }^{\circ} \mathrm{C}$. There are multiple adhesion measurement studies combining the cytodetachment method with the laser tweezer work station [105] and optical tweezer technique to further understand the temporal effects of cell adhesion by analyzing the molecular binding between the cell and the substrate [16,105]. The study of bovine articular chrondocyte cell adhesion with different ECM-coated substrate was carried out by using a combined cantilever glass beam with a carbon filament as a cytodetacher. Huang et al. [16] used rabbit articular chrondocyte cells to study the mechanical adhesiveness of the cells using the cytodetachment method. They examined membrane cell tether formation using optical tweezers and cytoskeleton change through fluorescent staining. Findings showed that chrondocytes exhibited increasing mechanical adhesiveness and tether formation force with the increment of seeding time. Yang et al. [106] reported that the adhesion force of human fetal osteoblast ( $h F O B)$ cells was influenced by the cell's shape grown on the Ca-P grooved micropattern surface.

Micropipette Aspiration Technique. Micropipette aspiration is a widely used technique for measuring the mechanical properties of single cells. For single cell adhesion measurement, this technique detaches an immobilized cell by applying suction force to a portion of the cell surface employed by micropipette suction [107] under observation via a microscope. The force will release the cell from the substrate by increasing the aspiration pressure or by translating the pipette away from the substrate [75]. Adhesion strength is defined as the minimum force needed to detach a single cell from its substrate. Micropipette aspiration technique is able to measure various mechanical properties of cells, such as the membrane stiffness of chondrocytes and endothelial cells [108], the cortical tension of neutrophils [108], and the adhesion strength of human umbilical vein endothelial cells on different substrates [109]. Gao et al. [109] used cells in the phase they called the "adhered cells in round shape" phase to eliminate the influences of floating cells, weakly adhered cells, and spread cells and obtained high reproducibility and sensitivity of the measurement on different substrates. They reported that the sensitivity and accuracy of their technique could reach $8 \times 10^{-12} \mathrm{~N}$ [109]. Micropipette aspiration technique was found able to identify comparable differences between the adhesion strength of normal and cancerous cells. Palmer et al. [18] have developed a single cell measuring apparatus using micropipette aspiration (SCAMA) that is able to measure and differentiate the adhesion strength of normal and cancerous prostate and breast cells. They found the measurement made on analogous human prostate cancer and normal cells showed a comparable three-fold difference in adhesiveness [18].

Single Cell Force Spectroscopy (SCFS). Force spectroscopy measurement methods were developed to measure the strength of cell adhesion down to single cell level. Commonly, the methods will use a microscope to observe the cell while force is applied to detach the cell using a nano/micromanipulator or micropipette. The imaging mode is used to study the structures and mechanics of isolated biomolecules [110-112], components of the cell nucleus [113,114], and 
subcellular cytoskeletal structures [110,115], while force mode is used to determine the mechanical properties of various cell types. The methods differ in the type of manipulation applied to the cell and the type of force measurements made. Examples of single cell force spectroscopy techniques used to measure cell detachment are the AFM probe techniques, biomembrane probe (BFP), and optical tweezer methods. Among these methods, AFM-based techniques are widely used to study various types of cell adhesion, effects of surface treatments and environmental conditions, and biomaterials compatibility.

AFM Probe Force Measurement. AFM probe force measurement is widely used to measure the stiffness [116-119] and adhesion strength of individual cells against mechanical force $[16,104,105,120]$ due to its versatility and precision. By immobilizing individual cells to an AFM cantilever, the living cell will be converted into a probe for the measurement of adhesion strength between cell-cell or cell-matrix adhesions [38]. This probe is attached to a cell or ECM-coated substrate (cell adhesion occurs) and the cantilever is withdrawn at a constant speed to detach the cell from its binding place. Cantilever deflection is recorded as force-distance curve and the highest force recorded represents the cell's adhesion strength [38]. When the probe encounters the single cell surface, various forces between the cantilever probe and cell lead to a deflection of the cantilever according to Hooke's law [120]. Adhesion strength of a single cell could be monitored as a function of adhesion time and environmental conditions by using AFM probe force measurement [75]. Various aspects of adhesion could be studied using AFM probe SCFS without restriction on the type of cell adhesion molecules and cell types used. Puench et al. [121] reported that the technique is able to quantitatively determine the adhesion of primary grastulating cells and provide insight into the role of Wnt11 signaling in modulating cell adhesion at single cell level. Weder et al. [122] studied the adhesion of human osteosarcoma cell lines (Saos-2) during different phases of the cell cycle and found that the cells are loosely attached to the substrate during the cells' round up ( $M$ phase) compared to during the interphase. Hoffmann et al. [123] determined the influence of the activating $N K$ cell receptor 2B4 on the early adhesion processes of $N K$ cells using AFM-probe SCFS. In addition, AFM is flexible and can be integrated with the standard modern inverted and transmission optical microscope. Lee et al. [105] developed a cell-detachment apparatus to measure the adhesion force of single cells integrated with a laser tweezer work station for cell manipulation observation. They studied the effect of experimental medium on the cell adhesion force of $N I H / 3 T 3$ fibroblast cells and found the cell adhesion strength increased with culturing time, and growth factor was found to enhance the adhesion strength between the cell and substrate. The study was continued with the combination of the optical trapping technique to further study the single cell adhesion properties of $M C D K$ cells in different phases of adhesion [119]. Their findings showed that focal adhesion kinase (FAK) plays a role in enhancing the binding and spreading of $M D C K$ cells through all the different phases of cell adhesion. Beaussart et al. [124] studied the adhesion forces of medically important microbes using AFM-SCFS and showed that procedures are applicable to pathogens such as Staphylococcus epidermidis and Candida albicans.

Biomembrane Force Probe (BFP). BFP is a sensitive technique that allows the quantification of single molecular bonds. It is a versatile tool that can be used in a wide range of forces $(0.1 \mathrm{pN}$ to $1 \mathrm{nN})$ and loading rates $\left(1-10^{6} \mathrm{pN} / \mathrm{s}\right)$ [125]. This technique uses a force transducer made of a 
biotinylated erythrocyte (such as biotinylated red blood cell (bRBC)) maintained by a glass micropipette. A streptavidin-coated glass microbead was attached to the bRBC (with known stiffness) and tuned by the controlled aspiration pressure applied by the holding micropipette. The assembly formed by the $\mathrm{bRBC}$ and the microbead constitutes a powerful nanodynamometer and is the force transducer (probe) used in the BFP [125]. The probe is brought into contact with the targeted cell and adhesion (bond formation) will occur between the probe and cell, followed by the detachment process where targeted cells are pulled away from the probe using a piezoelectric manipulator. Evans et al. [126,127] developed a transducer capable of measuring force ranging from $10^{-2}$ to $10^{3}$ picoNewton $(\mathrm{pN})$ for probing molecular adhesions and structures of a living cell interface [126] to improve their previous probing method [127]. The BFP technique was used to quantify the human neutrophil (PMN) membrane unbinding forces and the kinetics rate for the membrane unbinding was found to increase as an exponential function of the pulling force [128]. Gourier et al. [125] proved the capability of the technique to quantify the local changes of gamate (oocyte and spermatozoan) membrane adhesion and probe the mechanical behavior of the oocyte membrane at a micrometer scale.

Optical Tweezers (OT). OT uses a highly focused laser beam to trap and manipulate microscopic, neutral objects such as small dielectric spherical particles that experience two kinds of forces: namely, the scattering force and the gradient force $[129,130]$. The technique is able to measure forces ranging from sub-pN up to several hundreds of $\mathrm{pN}$ with good precision $(<1 \mathrm{pN})$ and is applicable for the study of interfacial interactions and non-covalent bonds, e.g., receptor-ligand bonds [129]. Single cell adhesion studies have been explored using OT [131-136] involving various cell types and purposes. Askenasy and Farkas [137] used OT for studying the cellular adhesion of hematopoietic stem cells (HSC) to the bone marrow stroma, and a forward scatter analysis (FORSA) has been integrated with the OT to investigate the binding force associated with cell-cell interactions and molecular interactions [132]. Thoumine et al. [131] were able to produce information on the receptor-ligand adhesion kinetics of fibroblast cells to fibronectin by coupling the experimental results with a probabilistic model of receptor-ligand kinetics. They gained information on the number, strength, and reaction rates of the bonds. Optical tweezers have also been applied to study the adhesion of Saccharomyces cerevisiae cells $[134,135,138]$ and to map the adhesion force during the formation and maturation of cell adhesion sites of mouse embryonic fibroblasts [136].

\section{Detachment Events: Population Approach}

Centrifugation Assay. Centrifugation assay is one of the frequently used techniques to measure cells adhesion strength due to their simplicity and the wide availability of equipment in most laboratories. Cells will be seeded in a multiwell plate and undergo treatments by culturing (cell culturing is similar to wash assay) before being spun for the cell detachment process. During the spinning, cells will experience a body force acting in the direction normal to the bottom of the plate that pulls them away from the surface [75]. To assess adhesion strength, the number of cells before and after application of load in the centrifuge is quantified. The fraction of cells that remains adhered after centrifugation can be determined by measuring the amount of radiation emitted from radio-labeled cells $[139,140]$, quantifying the amount of cells or cellular genetic material [139], or 
by using automated fluorescence analysis $[140,141]$. In many cases, the assay is used to assess the relative effect of treatments such as ECM protein type and concentration or the inhibition of a specific cellular function.

This method was used by Channavajjala et al. [141] to understand the importance of cell attachment to HIV-1 Tat protein and their finding shows that Tat protein mediates a significant but weak attachment of HT 1080 cells compared to the cells binding to ECM proteins. García et al. [142-145] used centrifugation assay to study cell adhesion and integrin binding and the effects of surface functionality of self-assembled. Harbers et al. [146,147] demonstrate the importance of flanking amino acids in the developing ligands with tuneable activity and the relative adhesion strength of each ligand by high-throughput assays for rapidly testing receptor-ligand engagement. High-throughput capabilities of the centrifugation method have been applied by Reyes and Garcia [144], who analyzed the adhesion capabilities of different cells (HT-1080, NIH3T3 and MC3T3-E1) on different concentrations of collagen or fibronectin. Koo et al. [140] used the method to study the effect of different ligand density and clustering to show that biophysical cues such as ligand spatial arrangement and ECM rigidity are central to the governance of cell responses to the external environment.

Spinning Disk. The spinning disk technique utilizes shear stress generated from a rotating disk device. Cells are first seeded on circular glass coverslipsor on the surface of a disk (typical diameter $10-50 \mathrm{~mm}$ ). These disks are later fixed onto a rotating device, that is placed inside a chamber filled with buffer solution $[75,148]$. The rotating device has a rotating range from 500 to $3000 \mathrm{rpm}$. The adherent fractions of cells are generally quantified using microscopy and by counting the number of cells before and after spinning using either a manual procedure [149] or automated image processing software [150]. The spinning disk technique has been used to investigate various types of cell-substrate interactions for wide range of applications such as quantifying the adhesion strength of an osteoblast-like cell on bioactive glass [150,151], human bone marrow cells on hydroxyapatite [152,153], and MC3T3-E1 cells on RGD peptides on self-assembled monolayers [154]. Lee et al. [154] used this method to quantify the nonspecific and specific contributions of bone cells on immobilized RGD peptides and quantitatively demonstrated both the possibilities and limitations of enhancing the osteogenic response of RGD-immobilized biomaterials by a change in peptide. The method has been used to study the role of focal adhesion kinase, which is essential for the focal adhesion function and the cell's adhesion strength [154,155]. Boettiger et al. observed the effect of transformed oncogene $v$-src on the adhesion strength of chick embryo fibroblasts [156] and human osteosarcoma cells [157] to understand its effect on integrin function. Lynch et al. [158] investigated the nature of signaling mechanisms that regulate integrin function in a steady-state adhesion and during cell motion using cells exposed to the insulin-like growth factor I $(I G F-I)$. The effect of surface charges on different substrates has also been studied using $H T-1080$ cells following fibronectin coating [159]. Reutelingsperger et al. [148] have investigated the effects of differential shear stresses on cell-cell and cell-matrix interactions in a monolayer of endothelial cells. García et al. [160] were able to measure the short- and long-term adhesion strength of IMR-90 human fibroblasts adhered to fibronectin-coated glass using the spinning disk technique. 
Flow Chamber. There are two types of flow chambers used for adhesion strength measurement: the radial and parallel flow chambers. The radial flow chamber methods involve flowing fluid in a chamber over adhered cells on a stationary substrate where a wide range of radially dependent shear stresses are applied [161] to detach a population of cells in a single experiment. The fluid flow is directed outwards from the center of a circular chamber, impinges on the surface of interest, and flows radially outward over a substrate seeded with cells. The inlet flow is directed outwards from the center of the chamber and the shear stress of the fluid decreases with increasing radial distance in a nonlinear fashion [75]. The radial flow chamber is also known as a stagnation point flow chamber or confined impinging jet [162]. The linear fluid velocity and shear stress will decreased radially across the disk as the flow duct cross-sectional area increases with the radius, and this method provides a continuous range of shear force in a single experiment [162].

The radial flow chamber technique has been used by Cozens-Roberts et al. [163] to investigate the effects of ligand and receptor densities and the influence of the $\mathrm{pH}$ and ionic strength of the medium on the cell-surface interactions. DiMilla et al. [164] studied the human smooth muscle cells (HSMCs) initial attachment strength and migration speed on a range of fibronectin and collagen type IV concentration. Their finding's suggested that cell-substrate initial attachment strength is a central variable governing cell migration speed and the cell's maximal migration occurs at an intermediate level of cell-substrate adhesiveness [164]. A number of studies have been carried out to study the adhesion strength of murine $3 T 3$ fibroblasts on the fibronectin-coated of self-assembled monolayers on glass surfaces [161,165-167]. The adhesion of MC3T3-E1 cells to multilayer polyallylamine hydrochloride (PAH) heparin films was analyzed in order to evaluate biocompatibility of various film chemistries [168]. Rezania et al. [169-173] carried out studies on the adhesion of osteoblasts and osteoblast-like cells to RGD peptides [169-171] and the adhesion of endothelial cells to interpenetrating polymer networks [173] for the application of orthopaedic implants. A study by Brown et al. [174] showed that low trypsin concentrations could improve cell adhesion and promote stronger endothelial adhesion while high trypsin concentrations significantly reduced the number of functional integrins available on the membrane. Beside human cells, the radial flow method has also been used to understand the adhesion kinetics of bacteria Escherichia coli $\mathrm{K} 12-\mathrm{D} 21$ in the in the mid-exponential and stationary growth phases under flow conditions. Chinese hamster ovary cells $(\mathrm{CHO})$ were used to explore the adhesion behavior towards different substrates with different treatments [175].

The parallel plate flow chamber consists of a bottom plate and an upper plate separated by a distance of the channel's height to form a rectangular flow channel. Cells are grown on a coverslip and positioned in the flow chamber, constructed by sandwiching a thin rubber gasket between two plates and mounted on a microscope to allow direct observation of the cells during experiments [75]. The flow is often driven using hydrostatic pressure from a raised reservoir or with an automated pump to independently drive the flow [176-178]. The fluid's shear stress can be adjusted by varying the flow's rate of the perfusate, the fluid viscosity, or the channel's height and width [179].

The parallel flow technique was first introduced to study endothelial cell adhesion [178,179] and has been further explored in the adhesion studies of biotinylated endothelial cells adhered on glass 
with fibronectin or RGD peptide functionalization [180-183]. Tapered height chamber have been developed to produce linear variations of shear stresses along channels at a single flow rate [184]. Cao et al. [185] modified the chamber to developed a side-view chamber system where side-view images of cellular deformation and adhesion to various adhesive surfaces under dynamic flow conditions could be observed. Cellular adhesion to surfaces functionalized with artificial ECM proteins and polymer surfaces treated by plasma using different gaseous substances have also been studied [186,187]. The parallel flow technique has been been used to investigate the adhesion potential of various cell types to a range of different materials, including poly-L-lactide (PLL) films [187-189], polyelectrolyte multilayer films [190], polyethylene films [191], and numerous glass-treated surfaces [177,178,192-194]. Interestingly, the flow chamber can be used to observed cells' vascular adhesion potential to the endothelial monolayer, which represents the human endothelial vein system. Gerszten et al. [176] were able to study the adhesion of monocytes to vascular cell adhesion molecule-1 (VCAM-1) transduced human endothelial cells under physiological flow conditions. Palange et al. [195] investigated the extravasation ability of circulating tumor cells (CTCs) to the endotheial cell layer under flow and the potential of natural compounds and curcumin treatment to attenuate the cell's metastasis potential.

Microfluidics. Microfluidic lab-on-a-chip technologies represent a revolution of the flow chamber in laboratory experimentation, bringing the benefit of miniaturization, integration, and automation to many research-based industries. These greatly reduce the size of the devices and make many portable instruments affordable with quick data read-outs. The use of small sample volumes leads to greater efficiency of chemical reagents, straightforward construction and operation processes, and low production costs per device, thereby allowing for disposability and fast sampling times. The ability for real-time observation makes microfluidics bring high promises for cell adhesion studies. In recent years, cell adhesion studies have been carried out in a miniature form of the traditional parallel plate flow chambers as discussed above, using flow in rectangular microchannels to apply shear stresses to cells. These devices are typically constructed from optically transparent PDMS bonded to glass using the soft lithography rapid prototyping process that allows many nearly identical devices to be manufactured in a short amount of time [196]. The optically transparent criteria is important to enable the use of different real-time microscopy techniques to explore cell behaviors under diverse experimental conditions [197]. Small dimensions associated with micrometer-sized channels ensure laminar flow even at very high linear fluid velocities, which is often required when large shear stresses are generated [198].

A microfluidic device consisting of eight parallel channels has been used to assess the effect of varying collagen and fibronectin concentrations on the adhesion strength of endothelial cells [197]. A series of microfluidic channels have been constructed to investigate the adhesion strength of fibroblast cells adhered to fibronectin-coated glass surfaces [198]. In another case, microchannel assays were used to examine the adhesion of various cell types on surfaces with various coatings, including collagen, glutaraldehyde, and silane [199]. Kwon et al. [19] used a microfluidic shear device consisting of four parallel channels with different surface topography patterns to separate cancer cells mixed in a population of healthy cells based on adhesion strength. A shear stress-dependent cell detachment from a temperature-responsive cell culture using a microfluidic device has been 
developed to quantitatively estimate the interaction between cells $(N I H / 3 T 3$ mouse fibroblast and bovine aeortic endothelial cells) and materials [200]. Recently, microfluidic technology has moved forward to the studies of single cells. Honarmandi et al. [24] integrated microfluidics with optical tweezers for the study of mechanotransduction and focal adhesion of single endothelial cells. Microfluidic devices were 'used to provide convenient means of positioning a cell into a specific location in the channel with controlled physiological conditions while the optical tweezers were used to detach the adhered cells. Christ et al. [107] have upgraded the application of microfluidics from the study of population cell adhesion to single cell studies. A rectangular microchannel was used to analyze the adhesion strength of single NIH3T3 fibroblast cells that had been allowed to adhere for $24 \mathrm{~h}$ on the collagen and fibronectin coatings on glass [107]. In this work, single cells were imaged throughout the detachment process, and the relationship between adhesion strength and cell geometry was investigated.

\section{Advantages and Limitations of the Techniques Used in Cell Adhesion Studies}

Single cell approaches allow for precise measurements of the separation of the cell from the substrate. Specialized equipment, which is bulky and expensive, is often required for manipulation and alignment of the probe and testing can be time-intensive. The single cell adhesion measurement approach provides more precise measurement of the individual cell when compared to the population cell approach. The single cell measurement approach allows the system to image biomolecules at nanometer-scale resolution, to have a dynamic range of forces able to be applied to cells, and to process samples in their physiological medium and aqueous buffer [120]. It has been widely used in the cells' teether (adhesion process) formation [126] and in the rupture forces $[125,128,201]$ of the molecular adhesion bonds that couldn't be measured by most of the cell adhesion strength measurement methods. However, beside the precision of the techniques, limitations such as low-throughput measurement, high equipment cost, time consumption, the need of a skilled operator, and other operator variables in the data obtained are unavoidable. These restrictions underscore the need for developing additional simple techniques that do not require expensive equipment, and are able to measure changes in cell adhesion properties associated with diseases or specific physiological perturbations. Some of the methods require computational processing and high-end confocal microscopes, which are not available in most laboratories. Table 2 summarizes the advantages and limitations of techniques used to study cell adhesion. The population cell approach provides data from the average response of a group of cells. Even though this approach could not provide precise data on the characteristic of an individual cell, the approach was still widely used to study cell adhesion until recently. The importance of the approach can not be denied as it provides essential information in the medical field for disease treatments, tissue engineering, and biomaterial compatibility. 
Table 2. Comparison of advantages and limitations in the techniques used for cell adhesion studies.

\begin{tabular}{|c|c|c|c|}
\hline Method & Strength & Weaknesses & References \\
\hline $\begin{array}{l}\text { Polyacylamide-traction } \\
\text { Force Microscopy } \\
\text { (PA-TFM) }\end{array}$ & $\begin{array}{l}\text { Real time observation; } \\
\text { No special and expensive equipment } \\
\text { needed for fabrication Inexpensive; } \\
\text { Flexible to chemical and } \\
\text { mechanical adjustment; } \\
\text { Adaptable to a large variety of cells }\end{array}$ & $\begin{array}{l}\text { Needs to record both unstressed and } \\
\text { stressed state of the substrate; } \\
\text { Suffers from uncertainties in tracking } \\
\text { beads' position }\end{array}$ & [47-54] \\
\hline $\begin{array}{l}\text { Micropatterning } \\
\text { (Micropost } \\
\text { array/micropillar) }\end{array}$ & $\begin{array}{l}\text { Real-time observation; } \\
\text { Force quantification easier and more } \\
\text { reliable than PA-TFM; } \\
\text { The micropillar stiffness is manipulated } \\
\text { by its geometry; } \\
\text { Gives good precision over surface } \\
\text { chemical properties on micrometer scale }\end{array}$ & $\begin{array}{l}\text { Substrate can alter cell's behavior; } \\
\text { Requires sophisticated equipment } \\
\text { to fabricate; } \\
\text { Needs skilled operator; } \\
\text { Sensitivity of the microposts to the } \\
\text { particular cell type needs to be } \\
\text { optimized }\end{array}$ & {$[26,55-60]$} \\
\hline $\begin{array}{l}\text { Three Dimensional } \\
\text { Traction Force } \\
\text { Quantification } \\
\text { (3D-TFM) }\end{array}$ & $\begin{array}{l}\text { Real-time observation; } \\
\text { Flexible to chemical and } \\
\text { mechanical adjustment; } \\
\text { Adaptable to a large variety of cells; } \\
\text { Flexible to chemical and } \\
\text { mechanical adjustment; } \\
\text { Adaptable to a large variety of cells }\end{array}$ & $\begin{array}{l}\text { Needs high-end confocal microscope; } \\
\text { Needs high computational } \\
\text { processing; } \\
\text { Needs to record both unstressed and } \\
\text { stressed state of the substrate; } \\
\text { Suffers from uncertainties in tracking } \\
\text { beads position }\end{array}$ & {$[61-70]$} \\
\hline Wash Assay & Simple & $\begin{array}{l}\text { Not a quantitative data, needs further } \\
\text { analysis to obtain quantitative data; } \\
\text { Poor reproducibility; } \\
\text { Insensitive }\end{array}$ & {$[71-74]$} \\
\hline Resonance Frequency & $\begin{array}{l}\text { Real-time observation; } \\
\text { Real-time measurement }\end{array}$ & Poor reproducibility & {$[36,76-95]$} \\
\hline Microfluidics & $\begin{array}{l}\text { Straightforward construction } \\
\text { and operation; } \\
\text { Real-time observation } \\
\text { and measurement; } \\
\text { Convenience in size } \\
\text { (compatible with cell sizes); } \\
\text { Fast and simple to operate; } \\
\text { Non-invasive to cell }\end{array}$ & $\begin{array}{l}\text { Low detachment force; } \\
\text { Restricted to short-term adhesion }\end{array}$ & $\begin{array}{l}\text { Attachment } \\
\text { events }[96-103,107] ; \\
\text { Detachment Events } \\
{[19,24,107] ;} \\
{[197-200]}\end{array}$ \\
\hline
\end{tabular}


Table 2. Cont.

\begin{tabular}{|c|c|c|c|}
\hline Method & Strength & Weaknesses & References \\
\hline Cytodetachment & $\begin{array}{l}\text { Real-time observation; Quick detachment } \\
\text { of cell; Range of force produced is high } \\
\text { and applicable to } \\
\text { long-term adhesion }\end{array}$ & $\begin{array}{l}\text { Alignment of probe and cell; } \\
\text { Time-consuming; } \\
\text { Needs highly skilled } \\
\text { (experienced) operator; } \\
\text { Operator variable; } \\
\text { Cell damage (hard contact); } \\
\text { Expensive equipment; } \\
\text { Not real-time measurement }\end{array}$ & [104-106] \\
\hline $\begin{array}{l}\text { Micropipette } \\
\text { Aspiration }\end{array}$ & $\begin{array}{l}\text { Real-time observation and measurement; } \\
\text { Common lab equipments }\end{array}$ & $\begin{array}{l}\text { Alignment of probe and cell; } \\
\text { High skilled (experienced) operator; } \\
\text { Operator variable; } \\
\text { Cell damage (hard contact) }\end{array}$ & {$[18,108-109]$} \\
\hline SCFS-AFM probe & $\begin{array}{l}\text { Real-time observation Precise data for } \\
\text { short term adhesion studies }\end{array}$ & $\begin{array}{l}\text { Alignment of probe and } \\
\text { cell require micromanipulator; } \\
\text { Time consuming; } \\
\text { Need skilled operator; } \\
\text { Operator variable; } \\
\text { Cell damage (hard contact); } \\
\text { Expensive equipments; } \\
\text { Not real-time measurement }\end{array}$ & [105]; [116-124] \\
\hline $\begin{array}{l}\text { SCFS-Biomembrane } \\
\text { Probe }\end{array}$ & $\begin{array}{l}\text { Real-time observation; Precise data for } \\
\text { short term adhesion studies }\end{array}$ & $\begin{array}{l}\text { Low maximum force }(\mathrm{pN}) \\
\text { Restricted to short term adhesion; } \\
\text { High skilled (experienced) operator; } \\
\text { Operator variable; } \\
\text { Probe variable (fluctuation of probe } \\
\text { due to thermal excitation) }\end{array}$ & {$[125-128]$} \\
\hline SCFS-Optical Tweezer & $\begin{array}{l}\text { Real-time observation; } \\
\text { Precise data for short term } \\
\text { adhesion studies; } \\
\text { Compatible with microfluidic device }\end{array}$ & $\begin{array}{l}\text { Low maximum force }(\mathrm{pN}) \\
\text { Restricted to short term adhesion; } \\
\text { High skilled (experienced) operator; } \\
\text { Operator variable; } \\
\text { Cell damage }\end{array}$ & [129-138] \\
\hline Centrifugation & $\begin{array}{l}\text { Many analysis can be examined in } \\
\text { parallel; Common lab equipments }\end{array}$ & $\begin{array}{l}\text { Low maximum force } \\
\text { (uncomplete detachment); } \\
\text { Only a single force can be applied } \\
\text { per experiment; } \\
\text { Nota real-time analysis }\end{array}$ & [139-147] \\
\hline Spinning Disk & $\begin{array}{l}\text { A range of stresses able to be applied in } \\
\text { single experiment; High stresses }\end{array}$ & $\begin{array}{l}\text { Not a real-time analysis; } \\
\text { Custom-made apparatuses }\end{array}$ & {$[148-160]$} \\
\hline $\begin{array}{l}\text { Flow chamber: Radial } \\
\text { flow; } \\
\text { Parallel flow }\end{array}$ & $\begin{array}{l}\text { Radial flow: Ranges of stresses applicable } \\
\text { in single experiment; Real-time cell } \\
\text { detachment observation; Paralel flow: } \\
\text { Simple fabrication; Straightforward } \\
\text { operation; Real-time cell } \\
\text { detachment observation }\end{array}$ & $\begin{array}{l}\text { Radial flow: Low detachment force; } \\
\text { Restricted to short term adhesion; } \\
\text { Paralel flow: Low detachment force; } \\
\text { Restricted to short term adhesion }\end{array}$ & $\begin{array}{l}{[163-175] ;} \\
{[176-195]}\end{array}$ \\
\hline
\end{tabular}




\section{Summary}

Studying human diseases from a biomechanical perspective can lead to a better understanding of the pathophysiology and pathogenesis of a variety of illnesses because changes occurring at the molecular level will affect, and can be correlated to, changes at the macroscopic level. Research on biomechanics at the cellular and molecular levels not only leads to a better elucidation of the mechanisms behind disease progression, it can also lead to new methods for early disease detection, thus providing important knowledge in the fight and treatments against the diseases. Sickle cell disease (SCD) could be characterized by observing the red blood cells' (RBC) adhesiveness and deformability [98,202-205]. Cell adhesiveness was found to be reduced in human cancers. Diseased cells' properties have been found to be physically different from that of healthy cells [206]. The adhesion strength of cancer cells was found to be lower than the normal cells [19] and decreased in line with their increased "metastatic potential" [18]. Reduced intercellular adhesiveness allows cancer cells to disobey the social order, resulting in the destruction of the histological structure, which is the morphological hallmark of malignant tumors [8]. Polymorphonuclear leukocytes (PMNs) migrate from the bloodstream to the inflammation sites by adhering to the surface of the endothelium during infection and tissue injury [207]. The knowledge obtained can also be useful in the development of new and improved assays and diagnostic devices, and the techniques are not only sensitive enough for the early detection of diseases, but they are also highly accurate, so it is possible to detect diseases when the symptoms or signs are hardly discernable. Determining chronic diseases in their initial stages is promising in curing the illness and saving lives, thus improving the quality of human health.

Figure 6 summarizes the importance of cell adhesion studies and its applications categorized by attachment and detachment events and grouped by single cell and population studies. Cell adhesion studies cover a wide range of important applications from the fundamental single cell adhesion behavior (morphology, migration, kinetics) and understanding the cell signaling pathway to how the physiological factors (temperature, $\mathrm{pH}$, fluid flow), treatments (chemical, drugs, toxic, different substrate) and conditions affect cell adhesion and cancer metastasis studies as well as tissue engineering and biocompatibility studies for implants. This essential information obtained from the adhesion studies leads to the development of the computational model for further studying and understanding cell adhesion [27,96,208-217]. The future potential of single cell adhesion characterization is especially significant for early disease diagnosis. This emerging field can lead to the development of biomarkers for chronic diseases and cancers in their early stage at the cellular level. Furthermore, the new techniques or devices will bring high promises in the search of suitable treatments for those who have diseases in their early stages. Beside the importance of single cell adhesion, the cell adhesion population approach plays an important role in and brings high promises for the development of biomaterials in tissue engineering for implantable bioMEMs/biosensors, tissue scaffold production, and the applications of artificial bones as well as tooth replacement. Cell adhesion population studies are also essential in analyzing the potential of drug treatments, improving drug delivery systems, attenuating cancer metastasis development, 
understanding the dynamic mechanism of cell adhesion in many important biological processes, and finding a cure for many diseases or human health-realated problems.

\section{Conclusions and Future Directions}

Cell adhesion is a very important process in the human biological system. Studying both cell attachment and detachment events provides essential knowledge in understanding many important functional processes in the human body, which lead us to find the causes and problems that trigger certain diseases and thus develop the strategy for curing and improving them. Many different techniques and adhesion assays have been developed to study cell adhesion applicable to a wide range of fields. Every method is unique and was developed for specific important and independent purposes, which makes them difficult to compare in finding the best method applicable for cell adhesion studies. Choosing an appropriate technique is highly dependent on the purpose of the information that a person desires to obtain. Both single cell and population studies are equally important and required to fully understand how cells behave and function in the human system.

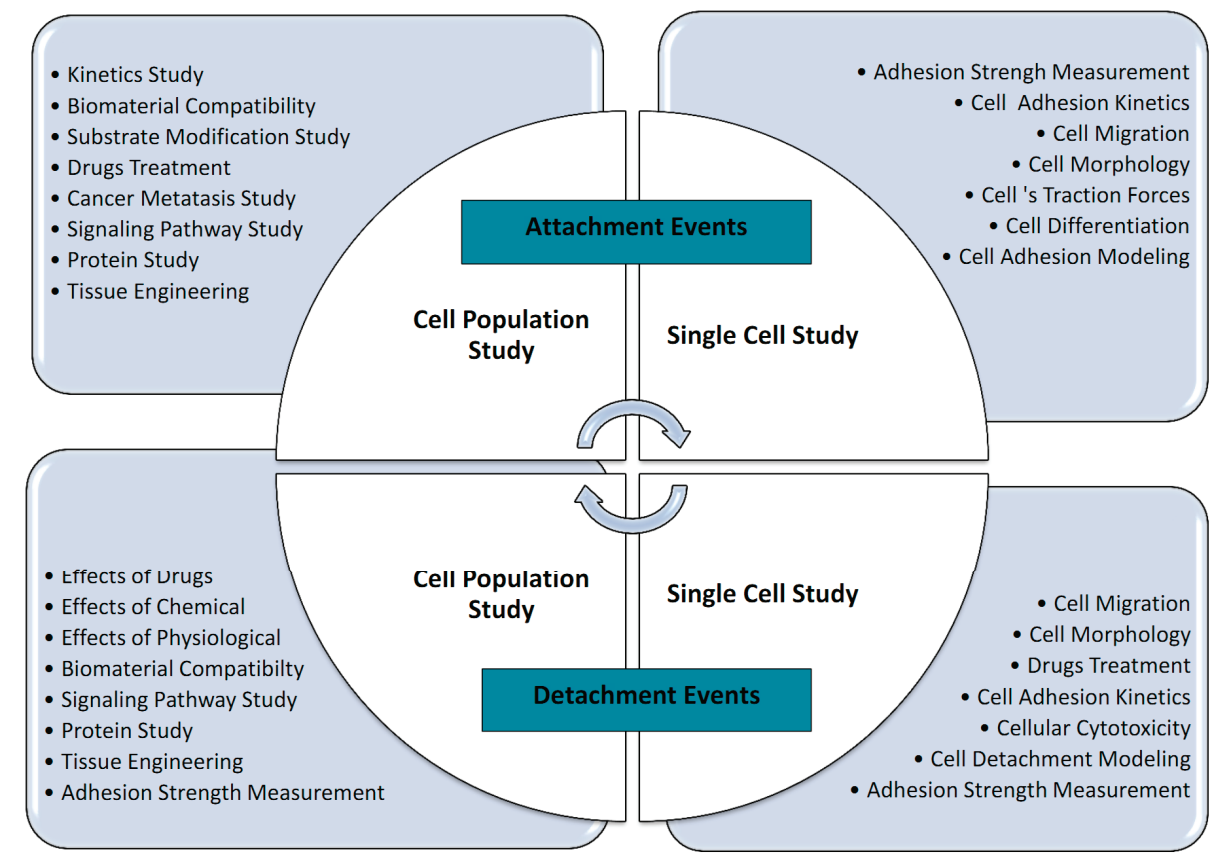

Figure 6. Summary of the importance of adhesion studies and their applications.

\section{Acknowledgments}

The research was supported by the Ministry of Higher Education Malaysia (grant Nos. 4L640 and 4F351) and Universiti Teknologi Malaysia, (grant Nos. 02G46, 03H82 and 03H80). Thanks to them for funding this project and for their endless support. 


\section{Author Contributions}

Amelia Ahmad Khalili and Mohd Ridzuan Ahmad wrote and edited the review, respectively.

\section{Conflicts of Interest}

The authors declare no conflict of interest.

\section{References}

1. Sagvolden, G.; Giaever, I.; Pettersen, E.O.; Feder, J. Cell adhesion force microscopy. Proc. Natl. Acad. Sci. USA 1999, 96, 471-476.

2. Dembo, M.; Torney, D.; Saxman, K.; Hammer, D. The kinetics of membrane-to-surface adhesion and detachment. Proc. R. Soc. 1988, 234, 55-83.

3. Shen, Y.; Nakajima, M.; Kojima, S.; Homma, M.; Kojima, M.; Fukuda, T. Single cell adhesion force measurement for cell viability identification using an AFM cantilever-based micro putter. Meas. Sci. Technol. 2011, 22, 115802.

4. Huang, S.; Ingber, D.E. The structural and mechanical complexity of cell-growth control. Nat. Cell Biol. 1999, 1, E131.

5. Lasky, L.A.; Singer, M.S.; Dowbenko, D.; Imai, Y.; Henzel, W.J.; Grimley, C.; Fennie, C.; Gillett, N.; Watson, S.R.; Rosent, S.D. An endothelial ligand for L-Selectin is a novel mucin-like molecule. Cell 1992, 69, 927-938.

6. Szekanecz, Z.; Koch, A.E. Cell-cell interactions in synovitis: Endothelial cells and immune cell migration. Arthritis Res. 2000, 2, 368-373.

7. Okegawa, T.; Pong, R.-C.; Li, Y.; Hsieh, J.-T. The role of cell adhesion molecule in cancer progression and its application in cancer therapy. Acta Biochim. Pol. 2004, 51, 445-457.

8. Hirohashi, S.; Kanai, Y. Cell adhesion system and human cancer morphogenesis. Cancer Sci. 2003, 94, 575-581.

9. Perinpanayagam, H.; Zaharias, R.; Stanford, C.; Keller, J.; Schneider, G.; Brand, R. Early cell adhesion events differ between osteoporotic and non-osteoporotic osteoblasts. J. Orthop. Res. 2001, 19, 993-1000.

10. Cho, P.; Schneider, G.B.; Kellogg, B.; Zaharias, R.; Keller, J.C. Effect of glucocorticoid-induced osteoporotic-like conditions on osteoblast cell attachment to implant surface microtopographies. Implant Dent. 2006, 15, 377-385.

11. Serhan, C.N.; Savill, J. Resolution of inflammation: The beginning programs the end. Nat. Immunol. 2005, 6, 1191-1197.

12. Simon, S.; Green, C.E. Molecular mechanics and dynamics of leukocyte recruitment during inflammation. Annu. Rev. Biol. 2005, 7, 151-185.

13. Spangenberg, C.; Lausch, E.U.; Trost, T.M.; Prawitt, D.; May, A.; Keppler, R.; Fees, S.A; Reutzel, D.; Bell, C.; Schmitt, S.; et al. ERBB2-mediated transcriptional up-regulation of the $\alpha 5 \beta 1$ integrin fibronectin receptor promotes tumor cell survival under adverse conditions. Cancer Res. 2006, 66, 3715-3725. 
14. Zou, J.X.; Liu, Y.; Pasquale, E.B.; Ruoslahti, E. Activated Src oncogene phosphorylates R-ras and suppresses integrin activity. J. Biol. Chem. 2002, 277, 1824-1827.

15. Mierke, C.T. Cancer cells regulate biomechanical properties of human microvascular endothelial cells. J. Biol. Chem. 2011, 286, 40025-40037.

16. Huang, W.; Anvari, B.; Torres, J.H.; LeBaron, R.G.; Athanasiou, K.A. Temporal effects of cell adhesion on mechanical characteristics of the single chondrocyte. J. Orthop. Res. 2003, 21, 88-95.

17. Wang, C.-C.; Hsu, Y.C.; Su, F.C.; Lu, S.C.; Lee, T.M. Effects of passivation treatments on titanium alloy with nanometric scale roughness and induced changes in fibroblast initial adhesion evaluated by a cytodetacher. J. Biomed. Mater. Res. 2009, 88, 370-383.

18. Palmer, C.P.; Mycielska, M.E.; Burcu, H.; Osman, K.; Collins, T.; Beckerman, R.; Perrett, R.; Johnson, H.; Aydar, E.; Djamgoz, M.B.A. Single cell adhesion measuring apparatus (SCAMA): Application to cancer cell lines of different metastatic potential and voltage-gated $\mathrm{Na}^{+}$channel expression. Eur. Biophys. J. 2008, 37, 359-368.

19. Kwon, K.W.; Choi, S.S.; Lee, S.H.; Kim, B.; Lee, S.N.; Park, M.C.; Kim, P.; Hwang, S.Y.; Suh, K.Y. Label-free, microfluidic separation and enrichment of human breast cancer cells by adhesion difference. Lab Chip 2007, 7, 1461-1468.

20. Saif, M.A.T.; Sager, C.R.; Coyer, S. Functionalized biomicroelectromechanical systems sensors for force response study at local adhesion sites of single living cells on substrates. Ann. Biomed. Eng. 2003, 31, 950-961.

21. Horwitz, A.F. Integrins and health. Sci. Am. 1997, 276, 68-75.

22. Chen, C.S. Geometric control of cell life and death. Science 1997, 276, 1425-1428.

23. Hwang, W.C.; Waugh, R.E. Energy of dissociation of lipid bilayer from the membrane skeleton of red blood cells. Biophys. J. 1997, 72, 2669-2678.

24. Honarmandi, P.; Lee, H.; Lang, M.J.; Kamm, R.D. A microfluidic system with optical laser tweezers to study mechanotransduction and focal adhesion recruitment. Lab Chip 2011, 11, 684-694.

25. Beningo, K.A.; Dembo, M.; Kaverina, I.; Small, J.V.; Wang, Y. Nascent focal adhesions are responsible for the generation of strong propulsive forces in migrating fibroblasts. J. Cell Biol. 2001, 153, 881-887.

26. Tan, J.L.; Tien, J.; Pirone, D.M.; Gray, D.S.; Bhadriraju, K.; Chen, C.S. Cells lying on a bed of microneedles: An approach to isolate mechanical force. Proc. Natl. Acad. Sci. USA 2003, 100, 1484-1489.

27. Zhong, Y.; He, S.; Ji, B. Mechanics in mechanosensitivity of cell adhesion and its roles in cell migration. Int. J. Comp. Mater. Sci. Eng. 2012, 01, 1250032.

28. Dumbauld, D.W.; Lee, T.T.; Singh, A.; Scrimgeour, J.; Gersbach, C.A.; Zamir, E.A.; Fu, J.; Chen, C.S.; Curtis, J.E.; Craig, S.W.; et al., How vinculin regulates force transmission. Proc. Natl. Acad. Sci. USA 2013, 110, 9788-9793.

29. Geiger, B.; Bershadsky, A.; Pankov, R.; Yamada, K.M. Transmembrane crosstalk between the extracellular matrix and the cytoskeleton. Nat. Rev. Mol. Cell Biol. 2001, 2, 793-805.

30. Hynes, R.O. Integrins: A family of cell surface receptors. Cell 1987, 48, 549-554. 
31. Van der Flier, A.; Sonnenberg, A. Function and interactions of integrins. Cell Tissue Res. 2001, 305, 285-298.

32. DiMilla, P.A.; Barbee, K.; Douglas, A.; Lauffenburger, D.A. Mathematical model for the effects of adhesion and mechanics on cell migration speed. Biophys. J. 1991, 60, 15-37.

33. Burridge, K.; Wennerberg, K.; Hill, C.; Carolina, N. Rho and Rac take center stage. Cell 2004, 116, 167-179.

34. Hall, A. Rho GTPases and the actin cytoskeleton. Science 1998, 279, 509-514.

35. LeBaron, R.G.; Athanasiou, K.A. Ex vivo synthesis of articular cartilage. Biomaterials 2000, 21, 2575-2587.

36. Hong, S.; Ergezen, E.; Lec, R.; Barbee, K.A. Real-time analysis of cell-surface adhesive interactions using thickness shear mode resonator. Biomaterials 2006, 27, 5813-5820.

37. Taubenberger, A.; Cisneros, D.; Friedrichs, J.; Puech, P.-H.; Muller, D.J.; Franz, C.M. Revealing early steps of alpha2beta1 integrin-mediated adhesion to collagen type I by using single-cell force spectroscopy. Mol. Biol. Cell 2007, 18, 1634-1644.

38. Helenius, J.; Heisenberg, C.-P.; Gaub, H.E.; Muller, D.J. Single-cell force spectroscopy. J. Cell Sci. 2008, 121, 1785-1791.

39. McEver, R.P.; Zhu, C. Rolling cell adhesion. Ann. Rev. Cell Dev. Biol. 2010, 26, 363-396.

40. Green, C.E.; Pearson, D.N.; Camphausen, R.T.; Staunton, D.E.; Simon, S.I. Shear-dependent capping of L-selectin and P-selectin glycoprotein ligand 1 by E-selectin signals activation of high-avidity $\beta 2$-integrin on neutrophils. J. Immunol. 2004, 172, 7780-7790.

41. Belloni, P.; Tressler, R. Microvascular endothelial cell heterogeneity: Interactions with leukocytes and tumor cells. Cancer Metastasis Rev. 1990, 8, 353-389.

42. Honn, K.; Tang, D. Adhesion molecules and tumor cell interaction with endothelium and subendothelial matrix. Cancer Metastasis Rev. 1992, 11, 353-375.

43. Ley, K.; Laudanna, C.; Cybulsky, M.I.; Nourshargh, S. Getting to the site of inflammation: The leukocyte adhesion cascade updated. Nat. Rev. Immunol. 2007, 7, 678-689.

44. Petri, B.; Phillipson, M.; Kubes, P. The physiology of leukocyte recruitment: An in vivo perspective. J. Immunol. 2008, 180, 6439-6446.

45. Imhof, B.A.; Aurrand-Lions, M. Adhesion mechanisms regulating the migration of monocytes. Nat. Rev. Immunol. 2004, 4, 432-444.

46. Muller, W.A. Leukocyte-endothelial-cell interactions in leukocyte transmigration and the inflammatory response. Trends Immunol. 2015, 24, 326-333.

47. Dembo, M.; Wang, Y. Stresses at the cell-to-substrate interface during locomotion of fibroblasts. Biophys. J. 1999, 76, 2307-2316.

48. Kraning-Rush, C.M.; Califano, J.P.; Reinhart-King, C.A. Cellular traction stresses increase with increasing metastatic potential. PLOS ONE 2012, 7, e32572.

49. Huynh, J.; Bordeleau, F.; Kraning-Rush, C.M.; Reinhart-King, C.A. Substrate stiffness regulates PDGF-induced circular dorsal ruffle formation through MLCK. Cell. Mol. Bioeng. 2014, 6, 1-16.

50. Reinhart-King, C.A.; Dembo, M.; Hammer, D.A. Endothelial cell traction forces on RGD-derivatized polyacrylamide substrata. Langmuir 2003, 19, 1573-1579. 
51. Sabass, B.; Gardel, M.L.; Waterman, C.M.; Schwarz, U.S. High resolution traction force microscopy based on experimental and computational advances. Biophys. J. 2008, 94, 207-220.

52. Ng, M.R.; Besser, A.; Danuser, G.; Brugge, J.S. Substrate stiffness regulates cadherin-dependent collective migration through myosin-II contractility. J. Cell Biol. 2012, 199, 545-563.

53. Wen, J.H.; Vincent, L.G.; Fuhrmann, A.; Choi, Y.S.; Hribar, K.C.; Taylor-weiner, H.; Chen, S.; Engler, A.J. Interplay of matrix stiffness and protein tethering in stem cell differentiation. Nat. Mater. 2014, 13, 979-984.

54. Califano, J.P.; Reinhart-King, C.A. Substrate stiffness and cell area predict cellular traction stresses in single cells and cells in contact. Cell. Mol. Bioeng. 2011, 3, 68-75.

55. Zhou, D.W.; García, A.J. Measurement systems for cell adhesive forces. J. Biomech. Eng. 2015, 137, 020908.

56. Théry, M. Micropatterning as a tool to decipher cell morphogenesis and functions. J. Cell Sci. 2010, 123, 4201-4213.

57. D'Arcangelo, E.; McGuigan, A.P. Micropatterning strategies to engineer controlled cell and tissue architecture in vitro. Biotechniques 2014, 58, 13-23.

58. Mandal, K.; Balland, M.; Bureau, L. Thermoresponsive micropatterned substrates for single cell studies. PLOS ONE 2012, 7, e37548.

59. Polio, S.R.; Rothenberg, K.E.; Stamenović, D.; Smith, M.L. A micropatterning and image processing approach to simplify measurement of cellular traction forces. Acta Biomater. 2012, $8,82-88$.

60. Ting, L.H.; Jahn, J.R.; Jung, J.I.; Shuman, B.R.; Feghhi, S.; Han, S.J.; Rodriguez, M.L.; Sniadecki, N.J. Flow mechanotransduction regulates traction forces, intercellular forces, and adherens junctions. Am. J. Physiol. 2012, 302, H2220-H2229.

61. Santos, E.; Hernández, R.M.; Pedraz, J.L.; Orive, G. Novel advances in the design of three-dimensional bio-scaffolds to control cell Fate: Translation from $2 \mathrm{D}$ to 3D. Trends Biotechnol. 2012, 30, 331-341.

62. Baker, B.M.; Chen, C.S. Deconstructing the third dimension: How 3D culture microenvironments alter cellular cues. J. Cell Sci. 2012, 125, 3015-3024.

63. Legant, W.R.; Miller, J.S.; Blakely, B.L.; Cohen, D.M.; Genin, G.M.; Chen, C.S. Measurement of mechanical tractions exerted by cells in three-dimensional matrices. Nat. Methods 2010, 7 , 969-971.

64. Khetan, S.; Guvendiren, M.; Legant, W.R.; Cohen, D.M.; Chen, C.S.; Burdick, J.A. Degradation-mediated cellular traction directs stem cell fate in covalently crosslinked three-dimensional hydrogels. Nat. Mater. 2013, 12, 458-465.

65. Meseke, M.; Förster, E. A 3D-matrigel/microbead assay for the visualization of mechanical tractive forces at the neurite-substrate interface of cultured neurons. J. Biomed. Mater. Res. A 2013, 101A, 1726-1733. 
66. Fraley, S.I.; Feng, Y.; Krishnamurthy, R.; Kim, D.-H.; Celedon, A.; Longmore, G.D.; Wirtz, D. A distinctive role for focal adhesion proteins in three-dimensional cell motility. Nat. Cell Biol. 2010, 12, 598-604.

67. Kraning-Rush, C.M.; Carey, S.P.; Califano, J.P.; Smith, B.N.; Reinhart-King, C.A. The role of the cytoskeleton in cellular force generation in 2D and 3D environments. Phys. Biol. 2011, $8,015009$.

68. Zaman, M.H.; Trapani, L.M.; Sieminski, A.L.; MacKellar, D.; Gong, H.; Kamm, R.D.; Wells, A.; Lauffenburger, D.A.; Matsudaira, P. Migration of tumor cells in 3D matrices is governed by matrix stiffness along with cell-matrix adhesion and proteolysis. Proc. Natl. Acad. Sci. USA 2006, 103, 10889-10894.

69. Bonakdar, N.; Butler, J.P.; Fabry, B.; Koch, T.M.; Mu, S. 3D traction forces in cancer cell invasion. PLOS ONE 2012, 7, e33476.

70. Kutys, M.L.; Yamada, K.M. An extracellular matrix-specific GEF-GAP interaction regulates Rho GTPase crosstalk for 3D collagen migration. Nat. Cell Biol. 2015, 16, 909-917.

71. Park, J.Y.; Arnaout, M.A.; Gupta, V. A simple, no-wash cell adhesion-based high-throughput assay for the discovery of small-molecule regulators of the integrin CD11b/CD18. J. Biomol. Screen. 2011, 12, 406-417.

72. Garcia, A.J.; Gallant, N.D. Stick and grip: Measurement systems and quantitative analyses of integrin-mediated cell adhesion strength. Cell Biochem. Biophys. 2003, 39, 61-73.

73. Chen, Y.; Lu, B.; Yang, Q.; Fearns, C.; Yates, J.R.; Lee, J.D. Combined integrin phosphoproteomic analyses and siRNA-based functional screening identified key regulators for cancer cell adhesion and migration. Cancer Res. 2010, 69, 3713-3720.

74. Mianabadi, M.; Yazdanparast, R. Inhibition of substrate-tumor cell adhesion under the effect of gnidilatimonoein purified from daphne mucronata. Am. J. Chin. Med. 2004, 32, 369-376.

75. Christ, K.V; Turner, K.T. Methods to measure the strength of cell adhesion to substrates. J. Adhes. Sci. Technol. 2010, 24, 37-41.

76. Ferreira, G.N.M.; da-Silva, A.-C.; Tomé, B. Acoustic wave biosensors: Physical models and biological applications of quartz crystal microbalance. Trends Biotechnol. 2009, 27, 689-697.

77. Wegener, J.; Janshoff, A. Cell adhesion monitoring using a quartz crystal microbalance: Comparative analysis of different mammalian cell lines. Eur. Biophys. J. 1998, 28, 26-37.

78. Heitmann, V.; Wegener, J. Monitoring cell adhesion by piezoresonators: Impact of increasing oscillation amplitudes. Anal. Chem. 2007, 79, 3392-3400.

79. Xi, J.; Chen, J.Y.; Garcia, M.P.; Penn, L.S. Quartz crystal microbalance in cell biology studies. J. Biochips Tissue Chipissue Chip. 2013, S5, 1-9.

80. Li, F.; Wang, J.H.-C.; Wang, Q.-M. Monitoring cell adhesion by using thickness shear mode acoustic wave sensors. Biosens. Bioelectron. 2007, 23, 42-50.

81. Fohlerová, Z.; Skládal, P.; Turánek, J. Adhesion of eukaryotic cell lines on the gold surface modified with extracellular matrix proteins monitored by the piezoelectric sensor. Biosens. Bioelectron. 2007, 22, 1896-1901.

82. Heitmann, V.; Rei $\beta$, B.; Wegener, J. The quartz crystal microbalance in cell biology: Basics and applications. Springer Ser. Chem. Sens. Biosens. 2007, 5, 303-338. 
83. Wegener, J.; Seebach, J.; Janshoff, A.; Galla, H.J. Analysis of the composite response of shear wave resonators to the attachment of mammalian cells. Biophys. J. 2000, 78, 2821-2833.

84. Modin, C.; Stranne, A.-L.; Foss, M.; Duch, M.; Justesen, J.; Chevallier, J.; Andersen, L.K.; Hemmersam, A.G.; Pedersen, F.S.; Besenbacher, F. QCM-D studies of attachment and differential spreading of pre-osteoblastic cells on Ta and $\mathrm{Cr}$ surfaces. Biomaterials 2006, 27, $1346-1354$.

85. Lord, M.S.; Modin, C.; Foss, M.; Duch, M.; Simmons, A.; Pedersen, F.S.; Milthorpe, B.K.; Besenbacher, F. Monitoring cell adhesion on tantalum and oxidised polystyrene using a quartz crystal Microbalance with dissipation. Biomaterials 2006, 27, 4529-4537.

86. Le Guillou-Buffello, D.; Hélary, G.; Gindre, M.; Pavon-Djavid, G.; Laugier, P.; Migonney, V. Monitoring cell adhesion processes on bioactive polymers with the quartz crystal resonator technique. Biomaterials 2005, 26, 4197-4205.

87. Zhu, Y.; Qiu, H.; Trzeciakowski, J.P.; Sun, Z.; Li, Z.; Hong, Z.; Hill, M.A; Hunter, W.C.; Vatner, D.E.; Vatner, S.F.; et al. Temporal analysis of vascular smooth muscle cell elasticity and adhesion reveals oscillation waveforms that differ with aging. Aging Cell 2012, 11, 741-750.

88. Hayward, L.; Thompson, M. Acoustic waves and the study of biochemical macromolecules and cells at the sensor-Liquid interface critical review. Analyst 1999, 124, 1405-1420.

89. Da-Silva, A.-C.; Soares, S.S.; Ferreira, G.N.M. Acoustic detection of cell Adhesion to a coated quartz crystal microbalance-Implications for studying the biocompatibility of polymers. Biotechnol. J. 2013, 8, 690-698.

90. Marx, K.A.; Zhou, T.; Montrone, A.; McIntosh, D.; Braunhut, S.J. Quartz crystal microbalance biosensor study of endothelial cells and their extracellular matrix following cell removal: Evidence for transient cellular stress and viscoelastic changes during detachment and the elastic behavior of the pure matrix. Anal. Biochem. 2005, 343, 23-34.

91. Marx, K.A.; Zhou, T.; Montrone, A.; McIntosh, D.; Braunhut, S.J. A comparative study of the cytoskeleton binding drugs nocodazole and taxol with a mammalian cell quartz crystal microbalance biosensor: Different dynamic responses and energy dissipation effects. Anal. Biochem. 2007, 361, 77-92.

92. Marx, K.A.; Zhou, T.; Montrone, A.; Schulze, H.; Braunhut, S.J. A quartz crystal microbalance cell biosensor: Detection of microtubule alterations in living cells at $\mathrm{nM}$ nocodazole concentrations. Biosens. Bioelectron. 2001, 16, 773-782.

93. Zhou, T.; Marx, K.A.; Warren, M.; Schulze, H.; Braunhut, S.J. The quartz crystal microbalance as a continuous monitoring tool for the study of endothelial cell surface attachment and growth. Biotechnol. Prog. 2000, 16, 268-277.

94. Warrick, J.W.; Young, E.W.K.; Schmuck, E.G.; Saupe, K.W.; Beebe, D.J. High-content adhesion assay to address limited cell samples. Integr. Biol. 2013, 5, 720-727.

95. Hartmann, A.; Stamp, M.; Kmeth, R.; Buchegger, S.; Stritzker, B.; Saldamli, B.; Burgkart, R.; Schneider, M.F.; Wixforth, A. A novel tool for dynamic cell adhesion studies-the De-Adhesion number investigator DANI. Lab Chip 2014, 14, 542-546. 
96. Gupta, V.K.; Sraj, I.A.; Konstantopoulos, K.; Eggleton, C.D. Multi-scale simulation of L-selectin-PSGL-1-dependent homotypic leukocyte binding and rupture. Biomech. Model. Mechanobiol. 2010, 9, 613-627.

97. Rupprecht, P.; Golé, L.; Rieu, J.-P.; Vézy, C.; Ferrigno, R.; Mertani, H.C.; Rivière, C. A tapered channel microfluidic device for comprehensive cell adhesion analysis, using measurements of detachment kinetics and shear stress-dependent motion. Biomicrofluidics 2012, 6, 14107-1410712.

98. Alapan, Y.; Little, J.A.; Gurkan, U.A. Heterogeneous red blood cell adhesion and deformability in sickle cell disease. Sci. Rep. 2014, 4, 7173.

99. Chaw, K.C.; Manimaran, M.; Tay, E.H.; Swaminathan, S. Multi-step microfluidic device for studying cancer metastasis. Lab Chip 2007, 7, 1041-1047.

100. Nalayanda, D.D.; Wang, Q.; Fulton, W.B.; Wang, T.-H.; Abdullah, F. Engineering an artificial alveolar-capillary membrane: A novel continuously perfused model within microchannels. J. Pediatr. Surg. 2010, 45, 45-51.

101. Song, J.W.; Cavnar, S.P.; Walker, A.C.; Luker, K.E.; Gupta, M.; Tung, Y.-C.; Luker, G.D.; Takayama, S. Microfluidic endothelium for studying the intravascular adhesion of metastatic breast cancer cells. PLOS ONE 2009, 4, e5756.

102. Fu, Y.; Kunz, R.; Wu, J.; Dong, C. Study of local hydrodynamic environment in cell-substrate adhesion using side-view $\mu$ PIV technology. PLOS ONE 2012, 7, e30721.

103. Riahi, R.; Yang, Y.L.; Kim, H.; Jiang, L.; Wong, P.K.; Zohar, Y. A microfluidic model for organ-specific extravasation of circulating tumor cells. Biomicrofluidics 2014, 8, 024103.

104. Yamamoto, A.; Mishima, S.; Maruyama, N.; Sumita, M. Quantitative evaluation of cell attachment to glass, polystyrene, and fibronectin- or collagen-coated polystyrene by measurement of cell adhesive shear force and cell detachment energy. J. Biomed. Mater. Res. 2000, 50, 114-124.

105. Lee, C. The technique for measurement of cell adhesion force. J. Med. Biol. Eng. 2004, 24, 51-56.

106. Yang, S.-P.; Yang, C.-Y.; Lee, T.-M.; Lui, T.-S. Effects of calcium-phosphate topography on osteoblast mechanobiology determined using a cytodetacher. Mater. Sci. Eng. 2012, 32, 254-262.

107. Christ, K.V.; Williamson, K.B.; Masters, K.S.; Turner, K.T. Measurement of single-cell adhesion strength using a microfluidic assay. Biomed. Microdevices 2010, 12, 443-455.

108. Hochmuth, R.M. Micropipette aspiration of living cells. J. Biomech. 2000, 33, 15-22.

109. Gao, Z.; Wang, S.; Zhu, H.; Su, C.; Xu, G.; Lian, X. Using selected uniform cells in round shape with a micropipette to measure cell adhesion strength on silk fibroin-based materials. Mater. Sci. Eng. C 2008, 28, 1227-1235.

110. Rotsch, C.; Jacobson, K.; Radmacher, M. Dimensional and mechanical dynamics of active and stable edges in motile fibroblasts investigated by using atomic force microscopy. Proc. Natl. Acad. Sci. USA 1999, 96, 921-926.

111. Muller, D.J. AFM: A nanotool in membrane biology. Biochemistry 2008, 47, 7896-7898. 
112. Engel, A.; Gaub, H.E. Structure and mechanics of membrane proteins. Annu. Rev. Biochem. 2008, 77, 127-148.

113. Hansma, H.G.; Kasuya, K.O.E. Atomic force microscopy imaging and pulling of nucleic acids. Curr. Opin. Struct. Biol. 2004, 14, 380-385.

114. Hirano, Y.; Takahashi, H.; Kumeta, M.; Hizume, K.; Hirai, Y.; Otsuka, S.; Yoshimura, S.H.; Takeyasu, K. Nuclear architecture and chromatin dynamics revealed by atomic force microscopy in combination with biochemistry and cell biology. Pflügers Archiv 2008, 456, 139-153.

115. Pesen, D.; Hoh, J.H. Micromechanical architecture of the endothelial cell cortex. Biophys. J. 2005, 88, 670-679.

116. Collinsworth, A.M.; Zhang, S.; Kraus, W.E.; Truskey, G.A. Apparent elastic modulus and hysteresis of skeletal muscle cells throughout differentiation. Am. J. Physiol. Cell Physiol. 2002, 283, C1219-C1227.

117. Solon, J.; Levental, I.; Sengupta, K.; Georges, P.C.; Janmey, P.A. Fibroblast adaptation and stiffness matching to soft elastic substrates. Biophys. J. 2007, 93, 4453-4461.

118. Rotsch, C.; Braet, F.; Wisse, E.; Radmacher, M. AFM imaging and elasticity measurements on living rat liver macrophages. Cell Biol. Int. 1997, 21, 685-696.

119. Wu, C.-C.; Su, H.-W.; Lee, C.-C.; Tang, M.-J.; Su, F.-C. Quantitative measurement of changes in adhesion force involving focal adhesion kinase during cell attachment, spread, and migration. Biochem. Biophys. Res. Commun. 2005, 329, 256-265.

120. Kim, W. A Micro-Aspirator Chip Using Vacuum Expanded Microchannels for High-Throughput Mechanical Characterization of Biological Cells; The Royal Society of Chemistry Publishing: London, UK, 2010; pp. 1-86.

121. Puech, P.-H.; Taubenberger, A.; Ulrich, F.; Krieg, M.; Muller, D.J.; Heisenberg, C.-P. Measuring cell adhesion forces of primary gastrulating cells from zebrafish using atomic force microscopy. J. Cell Sci. 2005, 118, 4199-4206.

122. Weder, G.; Vörös, J.; Giazzon, M.; Matthey, N.; Heinzelmann, H.; Liley, M. Measuring cell adhesion forces during the cell cycle by force spectroscopy. Biointerphases 2009, 4, 27-34.

123. Hoffmann, S.C.; Cohnen, A.; Ludwig, T.; Watzl, C. 2B4 engagement mediates rapid LFA-1 and actin-dependent NK cell adhesion to tumor cells as measured by single cell force spectroscopy. J. Immunol. 2011, 186, 2757-2764.

124. Beaussart, A.; El-Kirat-Chatel, S.; Sullan, R.M.A; Alsteens, D.; Herman, P.; Derclaye, S.; Dufrêne, Y.F. Quantifying the forces guiding microbial cell adhesion using single-cell force spectroscopy. Nat. Protoc. 2014, 9, 1049-1055.

125. Gourier, C.; Jegou, A.; Husson, J.; Pincet, F.A Nanospring named erythrocyte. The biomembrane force probe. Cell. Mol. Bioeng. 2008, 1, 263-275.

126. Evans, E.; Ritchie, K.; Merkel, R. Sensitive force technique to probe molecular adhesion and structural linkages at biological interfaces. Biophys. J. 1995, 68, 2580-2587.

127. Evans, E.; Berk, D.; Leung, A. Detachment of agglutinin-bonded red blood cells. I. Forces to rupture molecular-point attachments. Biophys. J. 1991, 59, 838-848. 
128. Evans, E.; Heinrich, V.; Leung, A.; Kinoshita, K. Nano- to microscale dynamics of P-selectin detachment from leukocyte interfaces. I. Membrane separation from the cytoskeleton. Biophys. J. 2005, 88, 2288-2298.

129. Zhang, H.; Liu, K.-K. Optical tweezers for single cells. J. R. Soc. Interface 2008, 5, 671-690.

130. Ashkin, A. Optical trapping and manipulation of neutral particles using lasers. Proc. Natl. Acad. Sci. USA 1997, 94, 4853-4860.

131. Thoumine, O.; Kocian, P.; Kottelat, A; Meister, J.J. Short-term binding of fibroblasts to fibronectin: Optical tweezers experiments and probabilistic analysis. Eur. Biophys. J. 2000, 29, 398-408.

132. Tsai, J.-W.; Liao, B.-Y.; Huang, C.-C.; Hwang, W.-L.; Wang, D.-W.; Chiou, A.E.T.; Lin, C.-H. Applications of optical tweezers and an integrated force measurement module for biomedical research. Int. Soc. Opt. Photonics 2000, 4082, 213-221.

133. Curtis, J.E.; Spatz, J.P. Getting a grip: Hyaluronan-mediated cellular adhesion. Int. Soc. Opt. Photonics 2004, 5514, 455-466.

134. Castelain, M.; Pignon, F.; Piau, J.-M.; Magnin, A.; Mercier-Bonin, M.; Schmitz, P. Removal forces and adhesion properties of saccharomyces cerevisiae on glass substrates probed by optical tweezer. J. Chem. Phys. 2007, 127, 135104.

135. Castelain, M.; Rouxhet, P.G.; Pignon, F.; Magnin, A.; Piau, J.-M. Single-cell adhesion probed in-situ using optical tweezers: A case study with saccharomyces cerevisiae. J. Appl. Phys. 2012, 111, 114701.

136. Schwingel, M.; Bastmeyer, M. Force mapping during the formation and maturation of cell adhesion sites with multiple optical tweezers. PLOS ONE 2013, 8, e54850.

137. Askenasy, N.; Farkas, D.L. Optical imaging of PKH-labeled hematopoietic cells in recipient bone marrow in vivo. Stem Cells 2002, 20, 501-513.

138. Castelain, M.; Pignon, F.; Piau, J.-M.; Magnin, A. The initial single yeast cell adhesion on glass via optical trapping and Derjaguin-Landau-Verwey-Overbeek predictions. J. Chem. Phys. 2008, 128, 135101.

139. Koo, L.Y.; Irvine, D.J.; Mayes, A.M.; Lauffenburger, D.A.; Griffith, L.G. Co-regulation of cell adhesion by nanoscale RGD organization and mechanical stimulus. J. Cell Sci. 2002, 115, 1423-1433.

140. Giacomello, E.; Neumayer, J.; Colombatti, A.P.R. Centrifugal assay for fluorescence-based cell adhesion adapted to the analysis of ex vivo cells and capable of determining relative binding strengths. Biotechniques 1999, 26, 764-766.

141. Channavajjala, L.S.; Eidsath, A.; Saxinger, W.C.A. Simple method for measurement of cell-substrate attachment forces: Application to HIV-1 Tat. J. Cell Sci. 1997, 110, 249-256.

142. Capadona, J.R.; Collard, D.M.; García, A.J. Fibronectin adsorption and cell adhesion to mixed monolayers of tri(ethylene glycol)- and methyl-terminated alkanethiols. Langmuir 2002, 19, 1847-1852.

143. Keselowsky, B.G.; García, A.J. Quantitative methods for analysis of integrin binding and focal adhesion formation on biomaterial surfaces. Biomaterials 2005, 26, 413-418. 
144. Reyes, C.D.; García, A.J. A centrifugation cell adhesion assay for high-throughput screening of biomaterial surfaces. J. Biomed. Mater. Res. A 2003, 67, 328-333.

145. Reyes, C.D.; Petrie, T.A.; García, A.J. Mixed extracellular matrix ligands synergistically modulate integrin adhesion and signaling. J. Cell. Physiol. 2008, 217, 450-458.

146. Harbers, G.M.; Healy, K.E. The effect of ligand type and density on osteoblast adhesion, proliferation, and matrix mineralization. J. Biomed. Mater. Res. A 2005, 75, 855-869.

147. Harbers, G.M.; Gamble, L.J.; Irwin, E.F.; Castner, D.G.; Healy, K.E. Development and characterization of a high-throughput system for assessing cell-surface receptor-ligand engagement. Langmuir 2005, 21, 8374-8384.

148. Reutelingsperger, C.P.M.; van Gool, R.G.J.; Heijnen, V.; Frederik, P.; Lindhout, T. The rotating disc as a device to study the adhesive properties of endothelial cells under differential shear stresses. J. Mater. Sci. Mater. Med. 1994, 5, 361-367.

149. Gallant, N.D.; Michael, K.E.; Garc1, J. Cell adhesion strengthening: Contributions of adhesive area , integrin binding, and focal adhesion assembly. Mol. Biol. Cell 2005, 16, 4329-4340.

150. García, A.J.; Ducheyne, P.; Boettiger, D. Quantification of cell adhesion using a spinning disc device and application to surface-reactive materials. Biomaterials 1997, 18, 1091-1098.

151. García, A.J.; Ducheyne, P.; Boettiger, D. Effect of surface reaction stage on fibronectin-mediated adhesion of osteoblast-like cells to bioactive glass. J. Biomed. Mater. Res. 1998, 40, 48-56.

152. Deligianni, D.D.; Katsala, N.D.; Koutsoukos, P.G.; Missirlis, Y.F. Effect of surface roughness of hydroxyapatite on human bone marrow cell adhesion, proliferation, differentiation and detachment strength. Biomaterials 2000, 22, 87-96.

153. Deligianni, D.; Korovessis, P.; Porte-Derrieu, M.C.; Amedee, J. Fibronectin preadsorbed on hydroxyapatite together with rough surface structure increases osteoblasts' adhesion "in vitro": The theoretical usefulness of fibronectin preadsorption on hydroxyapatite to increase permanent stability and longevity in spine implant. J. Spinal Disord. Tech. 2005, 18, 257-262.

154. Lee, M.H.; Adams, C.S.; Boettiger, D.; DeGrado, W.F.; Shapiro, I.M.; Composto, R.J.; Ducheyne, P. Adhesion of MC3T3-E1 cells to RGD peptides of different flanking residues: Detachment strength and correlation with long-term cellular function. J. Biomed. Mater. Res. A 2007, 81A, 150-160.

155. Michael, K.E.; Dumbauld, D.W.; Burns, K.L.; Hanks, S.K.; García, A.J. Focal adhesion kinase modulates cell adhesion strengthening via integrin activation. Mol. Biol. Cell 2009, 20, 2508-2519.

156. Shi, Q.; Boettiger, D. A novel mode for integrin-mediated signaling: Tethering is required for phosphorylation of FAK Y397. Mol. Biol. Cell 2003, 14, 4306-4315.

157. Datta, A.; Huber, F.; Boettiger, D. Phosphorylation of B3 integrin controls ligand binding strength. J. Biol. Chem. 2002, 277, 3943-3949.

158. Lynch, L.; Vodyanik, P.I.; Boettiger, D.; Guvakova, M.A. Insulin-like growth factor I controls adhesion strength mediated by $\alpha 5 \beta 1$ integrins in motile carcinoma cells. Mol. Biol. Cell 2005, 16, 51-63.

159. Miller, T.; Boettiger, D. Control of intracellular signaling by modulation of fibronectin conformation at the cell-materials interface. Langmuir 2002, 19, 1723-1729. 
160. García, A.J.; Takagi, J.; Boettiger, D. Two-stage activation for $\alpha 5 \beta 1$ integrin binding to surface-adsorbed fibronectin. J. Biol. Chem. 1998, 273, 34710-34715.

161. Goldstein, A.S.; DiMilla, P.A. Application of fluid mechanic and kinetic models to characterize mammalian cell detachment in a radial-flow chamber. Biotechnol. Bioeng. 1997, $55,616-629$.

162. Detry, J.G.; Deroanne, C.; Sindic, M. Hydrodynamic systems for assessing surface fouling, soil adherence and cleaning in laboratory installations. Biotechnol. Agron. Soc. Environ. 2009, $13,427-439$.

163. Cozens-roberts, C.; Quinn, J.A.; Lauffenburger, D.A. Receptor-mediated adhesion phenomena model studies with the radial-flow detachment assay. Biophys. J. 1990, 58, 107-125.

164. Dimilla, P.A.; Stone, J.A.; Albelda, S.M.; Biology, S. Maximal migration of human smooth muscle cells on fibronectin and type IV collagen occurs at an intermediate attachment strength. J. Cell Biol. 1993, 122, 729-737.

165. Goldstein, A.S.; DiMilla, P.A. Examination of membrane rupture as a mechanism for mammalian cell detachment from fibronectin-coated biomaterials. J. Biomed. Mater. Res. A 2003, 67A, 658-666.

166. Goldstein, A.S.; DiMilla, P.A. Effect of adsorbed fibronectin concentration on cell adhesion and deformation under shear on hydrophobic surfaces. J. Biomed. Mater. Res. 2002, 59, $665-675$.

167. Goldstein, A.S.; DiMilla, P.A. Comparison of converging and diverging radial flow for measuring cell adhesion. AIChE J. 1998, 44, 465-473.

168. Kreke, M.R.; Badami, A.S.; Brady, J.B.; Michael Akers, R.; Goldstein, A.S. Modulation of protein adsorption and cell adhesion by poly(allylamine hydrochloride) heparin films. Biomaterials 2005, 26, 2975-2981.

169. Rezania, A.; Healy, K.E. Biomimetic peptide surfaces that regulate adhesion, spreading, cytoskeletal organization, and mineralization of the matrix deposited by osteoblast-like cells. Biotechnol. Prog. 1999, 15, 19-32.

170. Rezania, A.; Healy, K.E. Integrin subunits responsible for adhesion of human osteoblast-like cells to biomimetic peptide surfaces. J. Orthop. Res. 1999, 17, 615-623.

171. Rezania, A.; Thomas, C.H.; Branger, A.B.; Waters, C.M.; Healy, K.E. The detachment strength and morphology of bone cells contacting materials modified with a peptide sequence found within bone sialoprotein. J. Biomed. Mater. Res. 1997, 37, 9-19.

172. Rezania, A.; Thomas, C.; Healy, K. A probabilistic approach to measure the strength of bone cell adhesion to chemically modified surfaces. Ann. Biomed. Eng. 1997, 25, 190-203.

173. Bearinger, J.P.; Castner, D.G.; Golledge, S.L.; Rezania, A.; Hubchak, S.; Healy, K.E. P(AAm-co-EG) interpenetrating polymer networks grafted to oxide surfaces: Surface characterization, protein adsorption, and cell detachment studies. Langmuir 1997, 13, 5175-5183.

174. Brown, M.A.; Wallace, C.S.; Anamelechi, C.C.; Clermont, E.; Reichert, W.M.; Truskey, G.A. The use of mild trypsinization conditions in the detachment of endothelial cells to promote subsequent endothelialization on synthetic surfaces. Biomaterials 2007, 28, 3928-3935. 
175. Sordel, T.; Kermarec-Marcel, F.; Garnier-Raveaud, S.; Glade, N.; Sauter-Starace, F.; Pudda, C.; Borella, M.; Plissonnier, M.; Chatelain, F.; Bruckert, F.; et al., Influence of glass and polymer coatings on $\mathrm{CHO}$ cell morphology and adhesion. Biomaterials 2007, 28, 1572-1584.

176. Gerszten, R.E.; Lim, Y.-C.; Ding, H.T.; Snapp, K.; Kansas, G.; Dichek, D.A.; Cabañas, C.; Sánchez-Madrid, F.; Gimbrone, M.A.; Rosenzweig, A.; et al. Adhesion of monocytes to vascular cell adhesion molecule-1-transduced human endothelial cells: Implications for atherogenesis. Circ. Res. 1998, 82, 871-878.

177. Truskey, G.A.; Pirone, J.S. The effect of fluid shear stress upon cell adhesion to fibronectin-treated surfaces. J. Biomed. Mater. Res. 1990, 24, 1333-1353.

178. Van Kooten, T.G.; Schakenraad, J.M.; van der Mei, H.C.; Busscher, H.J. Development and use of a parallel-plate flow chamber for studying cellular adhesion to solid surfaces. J. Biomed. Mater. Res. 1992, 26, 725-738.

179. Lane, W.O.; Jantzen, A.E.; Carlon, T.A; Jamiolkowski, R.M.; Grenet, J.E.; Ley, M.M.; Haseltine, J.M.; Galinat, L.J.; Lin, F.-H.; Allen, J.D.; et al. Parallel-plate flow chamber and continuous flow circuit to evaluate endothelial progenitor cells under laminar flow shear stress. $J$. Vis. Exp. 2012, 1-12.

180. Xiao, Y.; Truskey, G.A. Effect of receptor-ligand affinity on the strength of endothelial cell adhesion. Biophys. J. 1996, 71, 2869-2884.

181. Bhat, V.D.; Truskey, G.A.; Reichert, W.M. Using avidin-mediated binding to enhance initial endothelial cell attachment and spreading. J. Biomed. Mater. Res. 1998, 40, 57-65.

182. Chan, B.P.; Bhat, V.D.; Yegnasubramanian, S.; Reichert, W.M.; Truskey, G.A. An equilibrium model of endothelial cell adhesion via integrin-dependent and integrin-independent ligands. Biomaterials 1999, 20, 2395-2403.

183. Bhat, V.D.; Truskey, G.A.; Reichert, W.M. Fibronectin and avidin-biotin as a heterogeneous ligand system for enhanced endothelial cell adhesion. J. Biomed. Mater. Res. 1998, 41, 377-385.

184. Usami, S.; Chen, H.-H.; Zhao, Y.; Chien, S.; Skalak, R. Design and construction of a linear shear stress flow chamber. Ann. Biomed. Eng. 1993, 21, 77-83.

185. Cao, J.; Usami, S.; Dong, C. Development of a side-view chamber for studying cell-surface adhesion under flow conditions. Ann. Biomed. Eng. 1997, 25, 573-580.

186. Heilshorn, S.C.; DiZio, K.A.; Welsh, E.R.; Tirrell, D.A. Endothelial cell adhesion to the fibronectin CS5 domain in artificial extracellular matrix proteins. Biomaterials 2003, 24, 4245-4252.

187. Wan, Y.; Yang, J.; Yang, J.; Bei, J.; Wang, S. Cell adhesion on gaseous plasma modified poly-(1-lactide) surface under shear stress field. Biomaterials 2003, 24, 3757-3764.

188. Renshaw, K.M.; Orr, D.E.; Burg, K.J.L. Design and evaluation of a novel flow chamber for measuring cell adhesion to absorbable polymer films. Biotechnol. Prog. 2005, 21, 538-545.

189. Yang, J.; Wan, Y.; Yang, J.; Bei, J.; Wang, S. Plasma-treated, collagen-anchored polylactone: Its cell affinity evaluation under shear or shear-free conditions. J. Biomed. Mater. Res. A 2003, $67 A, 1139-1147$. 
190. Boura, C.; Muller, S.; Vautier, D.; Dumas, D.; Schaaf, P.; Claude Voegel, J.; François Stoltz, J.; Menu, P. Endothelial cell-Interactions with polyelectrolyte multilayer films. Biomaterials 2005, 26, 4568-4575.

191. Lee, J.H.; Lee, S.J.; Khang, G.; Lee, H.B. The effect of fluid shear stress on endothelial cell adhesiveness to polymer surfaces with wettability gradient. J. Colloid Interf. Sci. 2000, 230, 84-90.

192. Van Kooten T.G.; Schakenraad J.M.; van der Mei, H.C.; Busscher, H.J. Influence of Substratum Wettability on the Strength of Adhesion of Human Fibroblasts. Biomaterials 1992, 13, 897-904.

193. Truskey, G.A.; Proulx, T.L. Relationship between 3 T3 cell spreading and the strength of adhesion on glass and silane surfaces. Biomaterials 1993, 14, 243-254.

194. Vogel, J.; Bendas, G.; Bakowsky, U.; Hummel, G.; Schmidt, R.R.; Kettmann, U.; Rothe, U. The role of glycolipids in mediating cell adhesion: A flow chamber Study 1. Biochim. Biophys. Acta 1998, 1372, 205-215.

195. Palange, A.L.; di Mascolo, D.; Singh, J.; de Franceschi, M.S.; Carallo, C.; Gnasso, A.; Decuzzi, P. Modulating the vascular behavior of Mmetastatic breast cancer cells by curcumin treatment. Front. Oncol. 2012, 2, 161.

196. Xia, Y.; Whitesides, G.M. Soft lithography. Angew. Chem. Int. Ed. 1998, 37, 550-575.

197. Young, E.W.K.; Wheeler, A.R.; Simmons, C.A. Matrix-dependent adhesion of vascular and valvular endothelial cells in microfluidic channels. Lab Chip 2007, 7, 1759-1766.

198. Lu, H.; Koo, L.Y.; Wang, W.M.; Lauffenburger, D.A; Griffith, L.G.; Jensen, K.F. Microfluidic shear devices for quantitative analysis of cell adhesion. Anal. Chem. 2004, 76, 5257-5264.

199. Zhang, X.; Jones, P.; Haswell, S. Attachment and detachment of living cells on modified microchannel surfaces in a microfluidic-based lab-on-a-chip system. Chem. Eng. J. 2008, 135, S82-S88.

200. Tang, Z.; Akiyama, Y.; Itoga, K.; Kobayashi, J.; Yamato, M.; Okano, T. Shear stress-dependent cell detachment from temperature-responsive cell culture surfaces in a microfluidic device. Biomaterials 2012, 33, 7405-7411.

201. Merkel, R.; Nassoy, P.; Leung, A.; Ritchie, K.; Evans, E. Energy landscapes of receptor-ligand bonds explored with dynamic force spectroscopy. Nature 1999, 397, 50-53.

202. An, X.; Mohandas, N. Disorders of red cell membrane. Br. J. Haematol. 2008, 141, 367-375.

203. Mohandas, N.; Gallagher, P.G. Red cell membrane: Past, present, and future. Blood 2008, 112, 3939-3949.

204. Correlates, M. Sickle erythrocyte adherence to vascular endothelium morphologic correlates and the requirement for divalent cations and collagen-binding plasma proteins. J. Clin. Investig. 1985, 76, 1605-1612.

205. Hebbel, R.P.; Boogaerts, M.A.B.; Eaton, J.W.; Steinberg, M.H. Erythrocyte adherence to endothelium in sickle-cell anemia. N. Engl. J. Med. 1980, 302, 992-995.

206. Lee, G.Y.H.; Lim, C.T. Biomechanics approaches to studying human diseases. Trends Biotechnol. 2007, 25, 111-118. 
207. Girdhar, G.; Shao, J.-Y. Simultaneous tether extraction from endothelial cells and leukocytes: Observation, mechanics, and significance. Biophys. J. 2007, 93, 4041-4052.

208. Schlüter, D.K.; Ramis-Conde, I.; Chaplain, M.A.J. Multi-scale modelling of the dynamics of cell colonies: Insights into cell-adhesion forces and cancer invasion from in silico simulations. J. R. Soc. Interface 2014, 12, 20141080.

209. Deshpande, V.; Mrksich, M.; Mcmeeking, R.; Evans, A. A bio-mechanical model for coupling cell contractility with focal adhesion formation. J. Mech. Phys. Solids 2008, 56, $1484-1510$.

210. Cheung, L.S.-L.; Zheng, X.; Wang, L.; Baygents, J.C.; Guzman, R.; Schroeder, J.A.; Heimark, R.L.; Zohar, Y. Adhesion dynamics of circulating tumor cells under shear flow in a bio-functionalized microchannel. J. Micromech. Microeng. 2011, 21, 054033.

211. Kong, D.; Ji, B.; Dai, L. Nonlinear mechanical modeling of cell adhesion. J. Theor. Biol. 2008, 250, 75-84.

212. Hodges, S.R.; Jensen, O.E. Spreading and peeling dynamics in a model of cell adhesion. J. Fluid Mech. 2002, 460, 381-409.

213. Kong, D.; Ji, B.; Dai, L. Stability of adhesion clusters and cell reorientation under lateral cyclic tension. Biophys. J. 2008, 95, 4034-4044.

214. Nicolas, A.; Besser, A.; Safran, S.A. Dynamics of cellular focal adhesions on deformable substrates: Consequences for cell force microscopy. Biophys. J. 2008, 95, 527-539.

215. Gallant, N.D.; García, A.J. Model of integrin-mediated cell adhesion strengthening. J. Biomech. 2007, 40, 1301-1309.

216. Ronan, W.; Deshpande, V.S.; McMeeking, R.M.; McGarry, J.P. Numerical investigation of the active role of the actin cytoskeleton in the compression resistance of cells. J. Mech. Behav. Biomed. Mater. 2012, 14, 143-157.

217. Kong, D.; Ji, B.; Dai, L. Stabilizing to disruptive transition of focal adhesion response to mechanical forces. J. Biomech. 2010, 43, 2524-2529. 
MDPI AG

Klybeckstrasse 64

4057 Basel, Switzerland

Tel. +4161 6837734

Fax +41 613028918

http://www.mdpi.com/

IJMS Editorial Office

E-mail: ijms@mdpi.com

http://www.mdpi.com/journal/ijms 


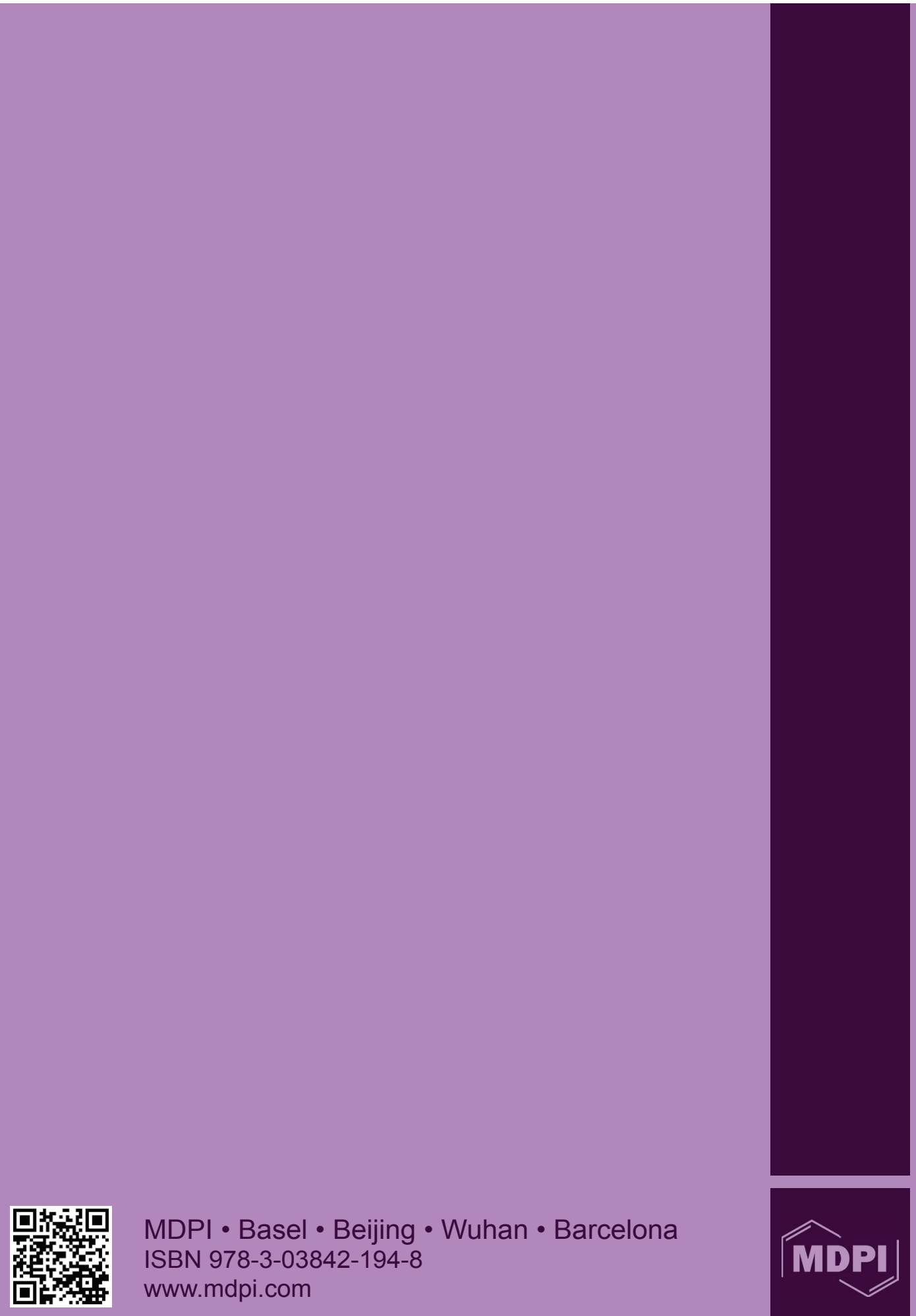